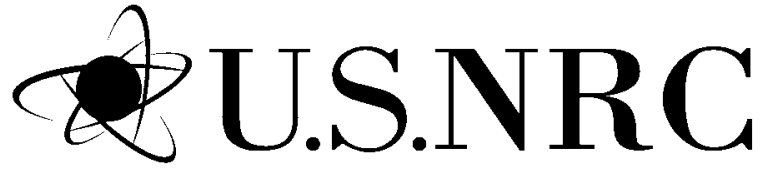

United States Nuclear Regulatory Commission

$\overline{\text { Protecting People and the Environment }}$

\title{
Mechanical Fatigue Testing of High-Burnup Fuel for Transportation Applications
}




\section{AVAILABILITY OF REFERENCE MATERIALS IN NRC PUBLICATIONS}

\section{NRC Reference Material}

As of November 1999, you may electronically access NUREG-series publications and other NRC records at NRC's Public Electronic Reading Room at http://www.nrc.gov/reading-rm.html. Publicly released records include, to name a few, NUREG-series publications; Federal Register notices; applicant, licensee, and vendor documents and correspondence; NRC correspondence and internal memoranda; bulletins and information notices; inspection and investigative reports; licensee event reports; and Commission papers and their attachments.

NRC publications in the NUREG series, NRC regulations, and Title 10, "Energy," in the Code of Federal Regulations may also be purchased from one of these two sources.

1. The Superintendent of Documents

U.S. Government Printing Office

Mail Stop SSOP

Washington, DC 20402-0001

Internet: bookstore.gpo.gov

Telephone: 202-512-1800

Fax: 202-512-2250

2. The National Technical Information Service Springfield, VA 22161-0002

www.ntis.gov

1-800-553-6847 or, locally, 703-605-6000

A single copy of each NRC draft report for comment is available free, to the extent of supply, upon written request as follows:

Address: U.S. Nuclear Regulatory Commission

Office of Administration

Publications Branch

Washington, DC 20555-0001

E-mail: DISTRIBUTION.RESOURCE@NRC.GOV

Facsimile: 301-415-2289

Some publications in the NUREG series that are posted at NRC's Web site address http://www.nrc.gov/reading-rm/doc-collections/nuregs are updated periodically and may differ from the last printed version. Although references to material found on a Web site bear the date the material was accessed, the material available on the date cited may subsequently be removed from the site.

\section{Non-NRC Reference Material}

Documents available from public and special technical libraries include all open literature items, such as books, journal articles, transactions, Federal Register notices, Federal and State legislation, and congressional reports. Such documents as theses, dissertations, foreign reports and translations, and non-NRC conference proceedings may be purchased from their sponsoring organization.

Copies of industry codes and standards used in a substantive manner in the NRC regulatory process are maintained at-

The NRC Technical Library

Two White Flint North

11545 Rockville Pike

Rockville, MD 20852-2738

These standards are available in the library for reference use by the public. Codes and standards are usually copyrighted and may be purchased from the originating organization or, if they are American National Standards, from-

American National Standards Institute

11 West $42^{\text {nd }}$ Street

New York, NY 10036-8002

www.ansi.org

212-642-4900

Legally binding regulatory requirements are stated only in laws; NRC regulations; licenses, including technical specifications; or orders, not in NUREG-series publications. The views expressed in contractorprepared publications in this series are not necessarily those of the NRC.

The NUREG series comprises (1) technical and administrative reports and books prepared by the staff (NUREG-XXXX) or agency contractors (NUREG/CR$X X X X),(2)$ proceedings of conferences (NUREG/CP$X X X X)$, (3) reports resulting from international agreements (NUREG/IA-XXXX), (4) brochures (NUREG/BR-XXXX), and (5) compilations of legal decisions and orders of the Commission and Atomic and Safety Licensing Boards and of Directors' decisions under Section 2.206 of NRC's regulations (NUREG0750).

DISCLAIMER: This report was prepared as an account of work sponsored by an agency of the U.S. Government. Neither the U.S. Government nor any agency thereof, nor any employee, makes any warranty, expressed or implied, or assumes any legal liability or responsibility for any third party's use, or the results of such use, of any information, apparatus, product, or process disclosed in this publication, or represents that its use by such third party would not infringe privately owned rights. 
United States Nuclear Regulatory Commission

$\overline{\text { Protecting People and the Environment }}$

\section{Mechanical Fatigue Testing of High-Burnup Fuel for Transportation Applications}

Manuscript Completed: August 2014

Date Published: May 2015

Prepared by

Jy-An Wang and Hong Wang

Oak Ridge National Laboratory

Managed by UT-Battelle, LLC

Oak Ridge, TN 37831-6069

Bruce Bevard, ORNL Program Manager

Michelle Flanagan-Bales, NRC Project Manager

NRC Job Codes N6686/N6789

Office of Nuclear Regulatory Research 



\section{ABSTRACT}

This report describes testing designed to determine the ability of high burnup (HBU) (>45 GWd/MTU) spent fuel to maintain its integrity under normal conditions of transportation. An innovative system, Cyclic Integrated Reversible-bending Fatigue Tester (CIRFT), has been developed at Oak Ridge National Laboratory (ORNL) to test and evaluate the mechanical behavior of spent nuclear fuel (SNF) under conditions relevant to storage and transportation. The CIRFT system is composed of a $U$-frame equipped with load cells for imposing the pure bending loads on the SNF rod test specimen and measuring the in-situ curvature of the fuel rod during bending using a set up with three linear variable differential transformers (LVDTs).

The HBU H.B. Robinson (HBR) SNF rods with Zircaloy-4 (Zry-4) cladding and intact fuel were studied under both static and dynamic (cyclic) bending. The HBR HBU rods survived static unidirectional bending to a maximum curvature of 3.1 to $3.5 \mathrm{~m}^{-1}$, or a maximum moment of 86 to $87 \mathrm{~N} \cdot \mathrm{m}$. The maximum longitudinal cladding offset strain before failure or before reaching CIRFT displacement capacity was 1.7 to $1.9 \%$, and the maximum bending stress was 733 to $748 \mathrm{MPa}$. The $0.2 \%$ offset yield stress of the HBU irradiated clad was estimated to be 687 to $727 \mathrm{MPa}$ based on the equivalent stress-strain curves.

In general, the static CIRFT test results indicate a significant increase in flexural rigidity compared to that of the defueled HBU rod specimen. Nevertheless, the segment composite structure of a HBU rod also introduces numerous stress concentration sites into a HBU rod system, ultimately resulting in $\mathrm{HBU}$ specimen fractured at the pellet-pellet interface regions under dynamic CIRFT fatigue testing.

The majority of the HBR dynamic test samples failed at or near a pellet-pellet interface. The fatigue life of HBU HBR rods during dynamic testing mainly depended on the level of loading and fuel pellet-clad interaction and the conditions of the rod. Under loading with moments of \pm 8.89 to $\pm 35.56 \mathrm{~N} \cdot \mathrm{m}$, resulting in an equivalent strain $\varepsilon$ of \pm 0.07 to $\pm 0.49 \%$ strain at $5 \mathrm{~Hz}$, the fatigue life $\mathrm{N}$ ranged from $5.5 \times 10^{3}$ to $2.3 \times 10^{6}$ cycles. Considering the complexity and nonuniformity of the HBU HBR fuel cladding system, it was significant to find that the $\varepsilon-\mathrm{N}$ data for the HBU HBR was characterized by a curve that would be expected of standard uniform materials. The $\varepsilon-\mathrm{N}$ curve of the HBU HBR rods can be described by a power function of $\mathrm{y}=$ $3.5693 \cdot x^{-0.252}$, where $x$ is the number of cycles to failure, and $y$ is the strain amplitude (\%). It was also significant to find that, if an endurance limit is defined by survival of $>10^{7}$ cycles, then the HBU HBR exhibited an endurance limit. The endurance limit for HBR HBU is likely located at approximately $0.1 \%$ strain.

The maxima of the imposed curvature $\kappa$ during dynamic testing ranged from \pm 0.16 to $\pm 1.19 \mathrm{~m}^{-1}$ at $5 \mathrm{~Hz}$. The $\kappa-\mathrm{N}$ curve of the HBU HBR rods can be described by a power function of $y$ $=8.1941 \cdot \mathrm{x}^{-0.252}$, where $\mathrm{x}$ is the number of cycles to failure, and $y$ is the maxima of clad tensile curvature $|\kappa|_{\max }\left(\mathrm{m}^{-1}\right)$. An endurance limit is likely located at approximately $0.2 \mathrm{~m}^{-1}$ when it is defined at $10^{7}$ cycles. 



\section{FOREWORD}

The U.S. Nuclear Regulatory Commission (NRC) requires the evaluation of nuclear fuel performance under normal and accident conditions, as specified in 10 CFR Parts 71 and 72, as part of spent nuclear fuel (SNF) storage and transportation cask certification. The certification of SNF storage and transportation systems requires a demonstration of safety and security. As part of demonstrating safety, the applicant must make an assessment about the condition of the SNF.

In the United States, SNF is expected to be transported to at least one storage facility before permanent disposal. During this transport phase, transportation casks will experience some level of oscillation associated with normal conditions of transport (NCT). This oscillation will be transmitted to the contents of the casks and the SNF assemblies, resulting in localized stresses. Nuclear fuel is now regularly irradiated to high-burnup (HBU) values ( $>45 \mathrm{GWd}$ /MTU). Several phenomena associated with HBU fuel have been identified, such as hydride reorientation that could impact the fatigue resistance of SNF under normal conditions of storage and transportation.

For these reasons, the fatigue resistance of HBU fuel should be investigated. The NRC has tasked Oak Ridge National Laboratory to investigate the flexural rigidity and fatigue life of HBU fuel. The testing was designed to evaluate the fuel rod as a system, including the presence of intact fuel inside the cladding and any fuel/cladding bonding effects. The properties measured in this testing program can be used in the evaluation of SNF integrity under NCT, when combined with details of an SNF cask design and expected transportation loading conditions.

The research effort was not intended to quantitatively evaluate SNF failure modes during NCT with respect to regulatory requirements. The results of this study are particular to the specific fuel tested. The results are not intended to be generically applicable and may not be inclusive of all cladding types and fuel burnup levels. 



\section{CONTENTS}

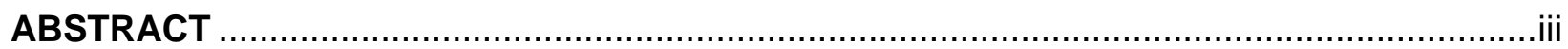

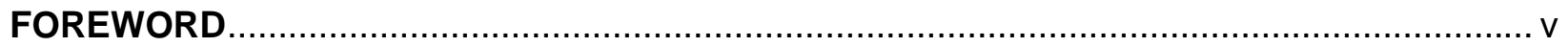

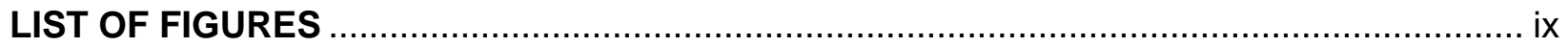

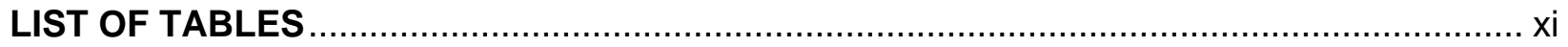

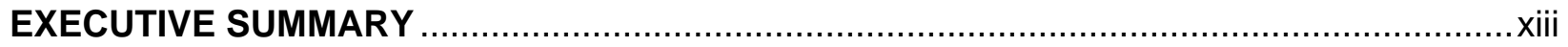

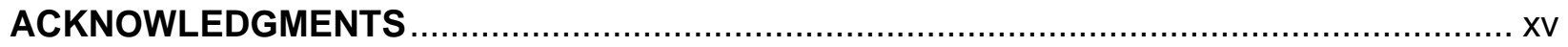

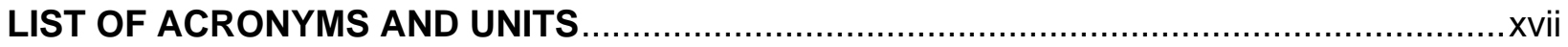

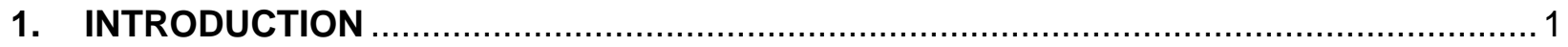

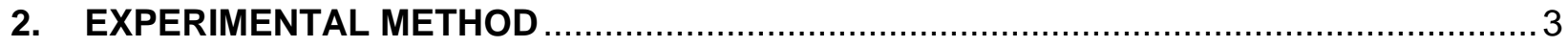

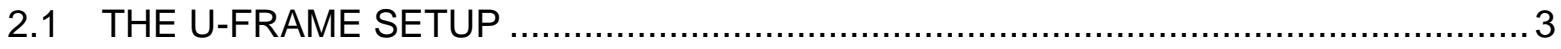

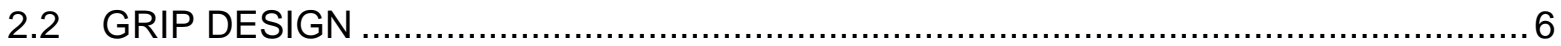

2.3 LVDT MEASUREMENTS AND CURVATURE CALCULATION .............................. 9

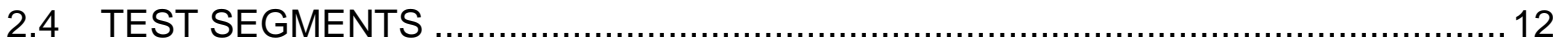

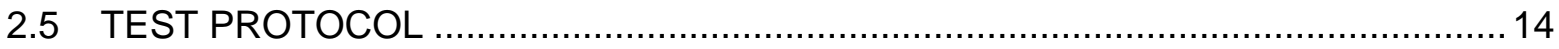

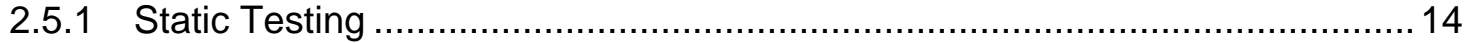

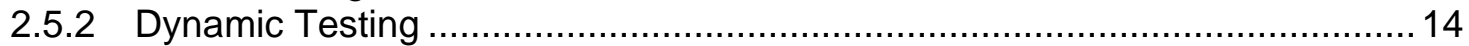

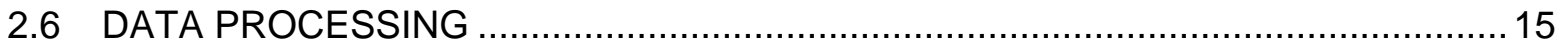

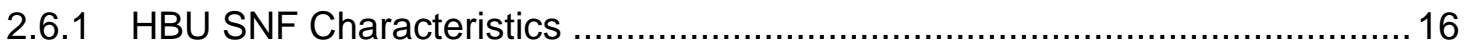

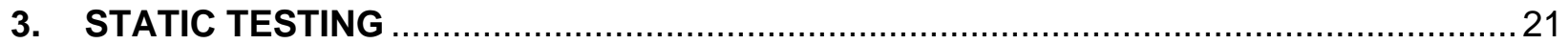

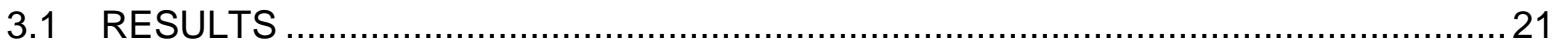

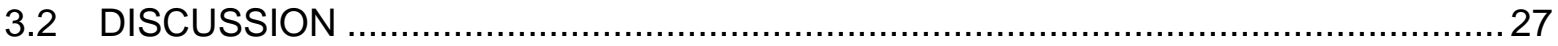

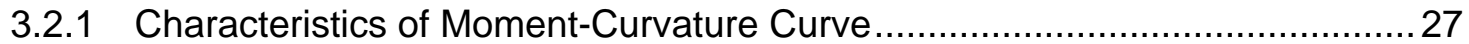

3.2.2 Comparison of Static Results with PNNL Cladding Data ............................... 30

3.2.3 Effect of LVDT Probe Contact on Large Curvature Measurement.................. 32

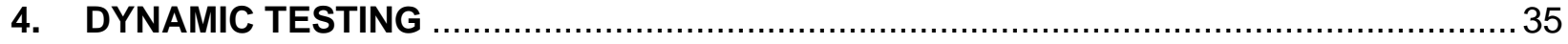

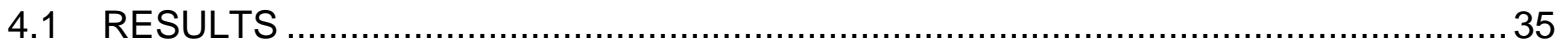

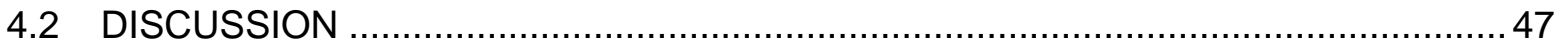

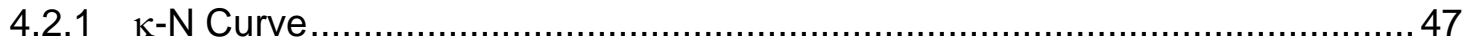

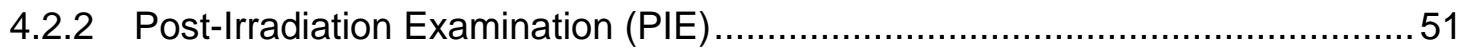

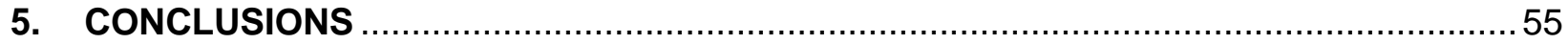

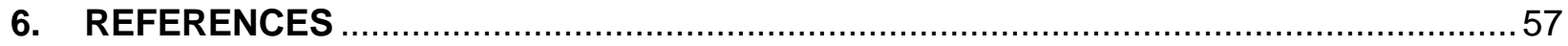

APPENDIX A...... 


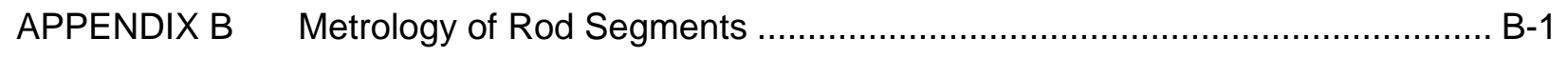

APPENDIX C Benchmarking of Test System ....................................................... C-1

APPENDIX D Static Test Results ........................................................................

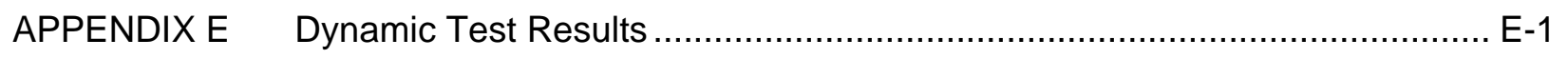

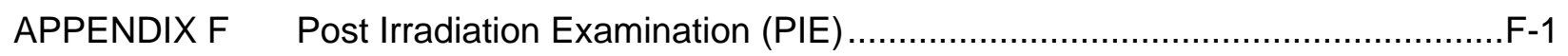




\section{LIST OF FIGURES}

Figure

Page

Figure 1. (a) Horizontal layout of ORNL U-frame setup; (b) rod specimen under test and three LVDTs for curvature measurement (operator is facing the three LVDTs); and (c) front view of CIRFT installed in ORNL hot cell..... 5

Figure 2. Schematic drawings of U-frame setup for reversal bending when rigid arms are (a) closing, (b) neutral, and (c) opening. 5

Figure 3. Assembled grip (left) and two views (right) of detached grip body...................... 6 Figure 4. Image showing the grip design of CIRFT with one end-block removed. 7

Figure 5. (a) Vise mold for assembling octagonal rigid sleeves into a rod; (b) rod (Demo1, 606B2) to be inserted; (c) one pin was turned away and a rigid sleeve loaded with epoxy inserted into the lower chamber.

Figure 6. Determination of the bending curvature of the rod by use of deflections measured at three points.

Figure 7. (a) Diagrams showing the effect of the LVDT probe with disk shape on curvature measurement, where the contact points are shifted.

Comparisons of curvature measurement between disk and chisel head probe for LVDTs located at clad compression and tension site are shown

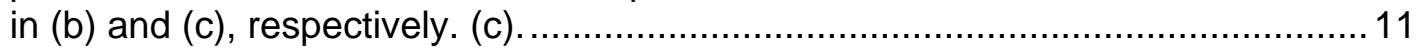

Figure 8. Segment $608 \mathrm{C} 4 \mathrm{~B}$ for DL2 taken from rod G10, with the storage tube................. 12

Figure 9. Flowchart for cyclic testing of spent fuel rod. .......................................... 15

Figure 10. Moment, curvature time series, and moment-curvature hysteresis loop for rod D1. Two measurements are displayed with relative displacement 0.8 and $1.2 \mathrm{~mm}$ at the loading points of U-frame.

Figure 11. "Empty-run" conducted on HBR S1, for which the load and displacement were plotted.

Figure 12. (a) Curve of moment versus curvature, and (b) equivalent strain-stress curve for S1 (606C3C).

Figure 13. Mating fracture surfaces (a) and (b); lateral sides near the neutral axis of the bending rod for S1 (606C3C) (c) and (d). The rod was subjected to four repeat static cycles to $24 \mathrm{~mm}$ maximum relative displacement followed by dynamic cyclic loading \pm 25.40 to $30.48 \mathrm{~N} \cdot \mathrm{m}$ at $5 \mathrm{~Hz} ; 1.4 \times 10^{4}$ failure cycles, 0.9 gram fuel particles collected.

Figure 14. Moment-curvature curves measurements in static tests (Corresponding stress and strain displayed on right and top axes, respectively).

Figure 15. Characteristic points of moment-curvature curve.

Figure 16. Comparison of CIRFT global data with PNNL moment-curvature curve converted from PNNL cladding stress-strain data.

Figure 17. Moment-curvature curves based on stainless steel alumina pellet (SSAP) rod testing when rod is bending with respect to three LVDTs (a) concavely and (b) convexly.

Figure 18. Moment-curvature curves as a function of sensor spacing adjustment when rod is bending with respect to three LVDTs (a) concavely and (b) convexly. The unit of $\mathrm{dh}$ is $\mathrm{mm}$.

Figure 19. Moment and curvature as a function of time and moment-curvature loops based on measurements when (a) $N=1$ and (b) $N=111,000$ cycles for D1 (607C4B). 
Figure 20. (a) Moment-curvature relation and (b) moment-flexural rigidity relation at various numbers of cycles for D1 (607C4B); $\mathrm{N}_{\mathrm{f}}=1.1 \times 10^{5}$ cycles under $\pm 15.24 \mathrm{~N} \cdot \mathrm{m}, 5 \mathrm{~Hz}$. Fuel particles collected $<1.0 \mathrm{~g}$....

Figure 21. Variations of (a) curvature range, (b) moment range, (c) flexural rigidity as a function of number of cycles for D1 (607C4B); $\mathrm{N}_{\mathrm{f}}=1.1 \times 10^{5}$ cycles under $\pm 15.24 \mathrm{~N} \cdot \mathrm{m}, 5 \mathrm{~Hz}$. Fuel particles collected $<1.0 \mathrm{~g}$.

Figure 22. Variations of (a) curvature range, (b) applied moment range, (c) flexural rigidity, (d) maximum and minimum values of curvature, (e) maximum and minimum values of moment, and ( $f$ ) flexural hysteresis as a function of number of cycles for D1 $(607 \mathrm{C} 4 \mathrm{~B}) ; \mathrm{N}_{\mathrm{f}}=1.1 \times 10^{5}$ cycles under \pm 15.24 $\mathrm{N} \cdot \mathrm{m}, 5 \mathrm{~Hz}$. Fuel particles collected $<1.0 \mathrm{~g}$....

Figure 23. Moment and curvature as a function of time and moment-curvature loops at (a) 26 and (b) $1.10 \times 10^{5}$ cycles; results are based on online monitoring.

Figure 24. (a) and (b) Mating fracture surfaces, (c) frontal, and (d) back sides for D1 (607C4B), $\mathrm{N}_{\mathrm{f}}=1.1 \times 10^{5}$ cycles under $\pm 15.24 \mathrm{~N} \cdot \mathrm{m}, 5 \mathrm{~Hz}$. Fuel particles collected $<1.0 \mathrm{~g}$.

Figure 25. Equivalent strain amplitudes $(\Delta \varepsilon / 2)$ as a function of number of cycles.

Figure 26. Equivalent strain amplitudes $(\Delta \varepsilon / 2)$ as a function of number of cycles with curve fitting extended to include the data points without failure. Solid markers represent tests with specimens failure; open markers indicate the tests stopped without failure.

Figure 27. (a) Maxima of absolute curvature extremes and curvature amplitudes as a function of number of cycles, (b) Maxima of absolute strain extremes and strain amplitudes as a function of number of cycles. (

Figure 28. (a) Curvature amplitudes, (b) maxima of absolute curvature extremes, and (c) maxima of absolute strain extreme as a function of number of cycles with curve-fitting extended to include the no-failure data points. 50

Figure 29. Optical images of untested segment 605D for (a) overall axial cross section and (b) enlarged area $A$.

Figure 30. (a) Axial cross section of S2/ 605D1E, (b) fracture surface near back side of rod and an enlarged area, and (c) fracture surface near front side of rod and an enlarged area. A - delamination, B - cracking of oxide layer. 


\section{LIST OF TABLES}

Table

Page

Table 1. Physical and mechanical properties of related materials ................................... 7

Table 2. Specifications of specimens used in hot-cell testing ….................................... 13

Table 3. Summary of static tests ............................................................................ 25

Table 4. Characteristic points and quantities based on curvature-moment curves ................29

Table 5. Characteristic points and quantities based on equivalent stress-strain curves .........29

Table 6. Comparison of flexural rigidity results in CIRFT tests and PNNL data .....................32

Table $7 . \quad$ Summary of dynamic test results ................................................................. 42

Table 8. Failure modes of rods from dynamic tests ................................................... 43 



\section{EXECUTIVE SUMMARY}

This report describes testing designed to determine the ability of high burnup (HBU) (>45 $\mathrm{GWd} / \mathrm{MTU}$ ) spent fuel to maintain its integrity under normal conditions of transportation.

In 2011, Oak Ridge National Laboratory (ORNL) proposed a bending fatigue system for testing HBU SNF rods. The proposed system is composed of a U-frame equipped with load cells for imposing the pure bending loads on the SNF rod test specimen and measuring the in-situ curvature of the fuel rod during bending using a set-up of three linear variable differential transformers (LVDTs).

By August 2013, ORNL had completed all the modifications needed for the testing system and the associated vise mold. On August 19, 2013, the completed testing system was installed in the Building 3525 hot cell and formally named the Cyclic Integrated Reversible-bending Fatigue Tester (CIRFT). After tuning the test system and benchmark testing in September 2013, ORNL completed four static tests under displacement control at the rate of $0.1 \mathrm{~mm} / \mathrm{s}$ to a maximum displacement of $12.0 \mathrm{~mm}$. The benchmark and static testing results were critically reviewed at the NRC-ORNL review meeting in early November 2013. The cyclic testing was then initiated. At this writing, 16 cyclic tests have been conducted in the hot cell. Load ranges varied from \pm 5.08 to $\pm 35.56 \mathrm{~N} \cdot \mathrm{m}$; there were 12 tests with failure and 4 tests without failure. One of the cyclic tests reached $1.3 \times 10^{7}$ cycles and was stopped.

In general, the static CIRFT results indicate a significant increase in flexural rigidity compared to the results for the defuel $\mathrm{HBU}$ rod specimen. Nevertheless, the segment composite structure of an HBU rod also introduces numerous stress concentration sites into an HBU rod system, ultimately resulting in $\mathrm{HBU}$ specimen fractures at the pellet-pellet interface regions under dynamic CIRFT fatigue testing.

The following observations were noted:

- The HBU HBR rods survived static unidirectional bending to a maximum curvature of 3.1 to $3.5 \mathrm{~m}^{-1}$, or a maximum moment of 86 to $87 \mathrm{~N} \cdot \mathrm{m}$. The maximum equivalent strain was 1.7 to $1.9 \%$, corresponding to equivalent stress of 733 to $748 \mathrm{MPa}$.

- The HBU HBR rods exhibited a multiple-stage constitutive response, with the two linear stages followed by a nonlinear stage. The flexural rigidity at the initial stage was 52 to 63 $\mathrm{N} \cdot \mathrm{m}^{2}$, corresponding to an elastic modulus of 83 to $101 \mathrm{GPa}$. The flexural rigidity at the second stage was 37 to $41 \mathrm{~N} \cdot \mathrm{m}^{2}$, and the corresponding elastic modulus was 59 to $66 \mathrm{GPa}$.

- $\quad$ The equivalent $0.2 \%$ yield strength of the HBU irradiated cladding was estimated to be 687 to $727 \mathrm{MPa}$ based on the equivalent stress-strain curves.

- Most of the failures of HBU HBR rods under static unidirectional loading were coincident with the pellet-to-pellet interface, as validated by the outcrop of pellet end faces in most of the fracture surfaces. Fragmentation of the pellets also occurred to a limited degree, along with cladding failure.

- The fatigue life of HBU HBR rods in the cyclic test depended on the level of loading. Under loading with moments of \pm 8.89 to $\pm 35.56 \mathrm{~N} \cdot \mathrm{m}$-namely \pm 0.07 to $\pm 0.49 \%$ strain at $5 \mathrm{~Hz}$ - the fatigue life ranged from $5.5 \times 10^{3}$ to $2.3 \times 10^{6}$ cycles. 
- The $\varepsilon$-N curve of the HBU HBR rods can be described by a power function of $y=$ $3.5693 \cdot x^{-0.252}$, where $x$ is the number of cycles to failure and $y$ is the strain amplitude (\%).

- Maxima of the imposed curvature during dynamic tests ranged from \pm 0.16 to $\pm 1.19 \mathrm{~m}^{-1}$ at 5 $\mathrm{Hz}$. The $\kappa-\mathrm{N}$ curve of the HBU HBR rods can be described by a power function of $\mathrm{y}=$ $8.1941 \cdot x^{-0.252}$, where $x$ is the number of cycles to failure and $y$ is the maxima of cladding tensile curvature $|\kappa|_{\max }\left(\mathrm{m}^{-1}\right)$. A fatigue limit is likely located between 0.226 and $0.245 \mathrm{~m}^{-1}$ if it is defined at $10^{7}$ cycles.

- The failure of HBU HBR rods under cyclic reverse loading often occurred near pellet-topellet interfaces, as validated by the outcrop of clean pellet end faces. 


\section{ACKNOWLEDGMENTS}

This research was sponsored by the U.S. Nuclear Regulatory Commission (NRC). The authors would like to thank Michelle Flanagan-Bales, NRC Program Manager, for providing guidance and support and Gordon Bjorkman, Bob Einziger, and Patrick Raynaud, NRC staff members, for providing invaluable input and comments during program development and execution.

The authors gratefully acknowledge Oak Ridge National Laboratory program managers Bruce Bevard and Rob Howard for their guidance; Ting Tan and Hao Jiang for providing finite element method simulation support; Chuck Baldwin for providing post-irradiation examination support; Brian Woody and Scot Thurman for providing hot-cell operation support; and Thomas Cox, Brian Sparks, and Randy Parten for providing drawing and machining support. 



\section{LIST OF ACRONYMS AND UNITS}

\begin{tabular}{|c|c|}
\hline CFR & Code of Federal Regulations \\
\hline CIRFT & Cyclic Integrated Reversible-bending Fatigue Tester \\
\hline EPRI & Electric Power Research Institute \\
\hline FEM & finite element method \\
\hline GWd & gigawatt-day \\
\hline HBR & H. B. Robinson \\
\hline $\mathrm{HBU}$ & high-burnup \\
\hline LVDT & linear variable differential transformer \\
\hline MTU & metric ton of uranium \\
\hline NCT & normal conditions of transport \\
\hline $\mathrm{N} \cdot \mathrm{m}$ & newton-meter \\
\hline NRC & U.S. Nuclear Regulatory Commission \\
\hline ORNL & Oak Ridge National Laboratory \\
\hline $\mathrm{PCl}$ & pellet-cladding interface \\
\hline PIE & post-irradiation examination \\
\hline PNNL & Pacific Northwest National Laboratory \\
\hline PPI & pellet-pellet interface \\
\hline PWR & pressurized water reactor \\
\hline SEM & scanning electron microscope \\
\hline SNF & spent nuclear fuel \\
\hline SS & stainless steel \\
\hline SSAP & stainless steel alumina pellet \\
\hline Zry-4 & Zircaloy-4 \\
\hline $\mathrm{d} 1, \mathrm{~d} 2, \mathrm{~d} 3$ & deflection of rod at LVDT1, LVDT2, LVDT3, mm \\
\hline E1, E2, E3 & elastic modulus, GPa \\
\hline El, El1, El2, El3 & flexural rigidity, $\mathrm{N} \cdot \mathrm{m}^{2}$ \\
\hline $\mathrm{F}$ & load, N \\
\hline h, h1, h2 & sensor spacing, m \\
\hline l & moment of inertia, $\mathrm{m}^{4}$ \\
\hline IC & moment of inertia for clad, $\mathrm{m}^{4}$ \\
\hline Ip & moment of inertia for pellet, $\mathrm{m}^{4}$ \\
\hline $\mathrm{L}$ & loading arm, m \\
\hline$\kappa, \kappa_{\mathrm{A}}, \kappa_{\mathrm{B}}, \kappa_{\mathrm{C}}$ & curvature, $\mathrm{m}^{-1}$ \\
\hline$|k|_{\max }$ & maximum clad tensile curvature, \\
\hline$M, M_{A}, M_{B}, M_{C}$ & moment, $\mathrm{N} \cdot \mathrm{m}$ \\
\hline
\end{tabular}


$\Delta \mathrm{M}$

$\Delta \kappa$

$\sigma, \sigma_{\mathrm{A}}, \sigma_{\mathrm{B}}, \sigma_{\mathrm{C}}$

$\varepsilon, \varepsilon_{\mathrm{A}}, \varepsilon_{\mathrm{B}}, \varepsilon_{\mathrm{C}}$

$\mathrm{y}_{\max }$ moment range, $\mathrm{N} \cdot \mathrm{m}$

curvature range, $\mathrm{m}^{-1}$

engineering stress, $\mathrm{MPa}$

engineering strain, \%

maximum y of cross section of rod, $m$ 


\section{INTRODUCTION}

Spent nuclear fuel (SNF) is expected to be transported at least once before permanent disposal. During this transportation phase, transport casks will experience a certain level of oscillation due to normal conditions of transport (NCT). This oscillation will be transmitted to the contents of the casks and SNF assemblies, resulting in localized stresses in the fuel rods. SNF is now regularly irradiated to high-burnup (HBU) values (>45 GWd/MTU). Several phenomena associated with HBU fuel have been identified, such as hydride reorientation that could influence cladding integrity. Increased burnup, the use of advanced materials, and variability in material properties may also influence fuel performance during transportation., ${ }^{1,2}$

For these reasons, the response of SNF to NCT is being investigated. The U.S. Nuclear Regulatory Commission (NRC) has tasked Oak Ridge National Laboratory (ORNL) to investigate the bending stiffness and fatigue life of HBU fuel during transportation. The testing was designed to evaluate the fuel rod as a system, including the presence of fuel pellets and any fuel/cladding bonding effects. The objective is to report the results of vibration fatigue testing of HBU SNF rods and the effect of the presence of the fuel pellets on the overall behavior of the fuel rod.

Testing was conducted on HBU SNF with Zircaloy-4 (Zry-4) cladding from a pressurized-water reactor (PWR). Using fuel rod segments from 5 separate rods, 4 static tests were conducted, followed by 16 cyclic fatigue tests in a range of load amplitudes. The burnup of the tested rod segments was ranged between 63.8 and $66.8 \mathrm{GWd} / \mathrm{MTU}$. The estimated oxide layer thickness on the waterside surface was approximately 40 to $110 \mu \mathrm{m}$, and corresponding hydrogen contents of hydrides within the cladding wall was estimated between 360 and 800 wppm. All of the fuel rod segments were tested in an as-received status in which there is no oxide layer removal, and most hydrides are in the circumferential orientation. Because both circumferential and radial hydrides are oriented in the planes parallel to the principal normal tensile stress during bending loading, it is expected that SNF rod fatigue strength may not be sensitive to the hydride orientation if there is no significant clad internal pressure. Nevertheless, the potential effect of radial hydride reorientation on the bending fatigue response of the HBU fuel rods is being investigated and will be reported in future.

The static tests defined the elastic and plastic responses of the fuel rod system, and the dynamic tests defined the fatigue life of the fuel rod system under constant loads. The effects of cyclic loading over a wide range of loading amplitudes were tested and evaluated to develop an understanding of the fatigue life of $\mathrm{HBU}$ fuel. Relations of equivalent strain versus the number of cycles to failure $(\varepsilon-N)$ or curvature versus the number of cycles to failure $(\kappa-N)$ were developed to estimate the influence of the imposed bending on the fatigue strength. The impacts of important fuel rod phenomena such as fuel-to-cladding bonding efficiency and the pellet-topellet pinning interaction were recognized. The effects of the stress concentration at pellet-pellet interfaces and SNF system interfacial bonding status, including perfect bonding and de-bonding, on the global flexural rigidity and the local plastic deformation were studied by using finite element method (FEM), and the implication of numerical results on the interpretation of bending testing was discussed. 



\section{EXPERIMENTAL METHOD}

The objective of this testing program is to develop testing protocols to evaluate the bending deformation responses and the associated fatigue mechanical properties for HBU fuel rods relevant to transportation conditions. Thus, the HBU fuel rods would be tested in a condition and geometry as close as possible to normal conditions of transport (NCT). Currently, most of the mechanical testing devices require a specified specimen design, such as a pre-notch or reduced gage section, to prevent failure at the grip location and ensure meaningful data. For this testing program, it was recommended that the test segment not be altered by machining a gage section. Therefore, an innovative U-frame and grip design were developed. Key features of the U-frame and grip design will be discussed in the sections that follow. Cost considerations also had a significant influence on the development of the testing program. Hot-cell space and time are extremely limited and costly, as is testing material. For this testing program, we wanted to use a small test segment, design a compact test device, and develop a test protocol for efficient testing. Important features of the test segments and test protocol will be discussed later in this report. Finally, the curvature response of the test segment needed to be measured with as little interpretation as possible. It was not practical to weld strain gauges onto the segments or use lasers to measure deflection on HBU material in a hot cell. Therefore, three adjacent linear variable differential transformers (LVDTs) were used to measure lateral deflections of the test specimen so that the curvature of the specimen could be calculated without interpretation or assumptions regarding the deformation of various components of the testing system. The important aspects of the three LVDTs and the calculation of specimen curvature are discussed in Section 2.3.

\subsection{THE U-FRAME SETUP}

Testing of the SNF rod specimen was accomplished using a cyclic integrated reversible-bending fatigue tester (CIRFT) recently developed by ORNL. ${ }^{3,4,5,6,7,8,9,10,11}$ The integrated U-frame is shown in Figure 1.

The U-frame includes two rigid arms, connecting plates, and universal testing machine links. The rod specimen is coupled to the rigid arms through two specially designed grips. The Uframe setup is oriented in a horizontal plane and is driven by electromagnetic-force-based Bose dual linear motors. With help from the coupling, linear motions applied at the loading points of the rigid arms are converted into bending moments exerted on the rod. The dual linear motor (model LM2) test bench has a maximum load capacity of $\pm 3,000 \mathrm{~N}$ and a maximum stroke of $\pm 25.6 \mathrm{~mm}$.

Bending is imposed through a U-frame with dual driving points and a $101.60 \mathrm{~mm}$ loading arm. Under a pair of forces or displacements that face outward, the rigid arms are opened, and bending moments force the rod to deflect outward (namely, away from operator, as shown in Figure 2). Under a pair of forces facing each other, the rigid arms are closed, forcing the rod to deflect inward. With the special grips that will be described in Section 2.2, a pure reversible bending condition can be obtained. The CIRFT can deliver dynamic loading to a rod specimen in the load-control mode at 5 to $10 \mathrm{~Hz}$. The current configuration enables the system to test a rod 9.70 to $11.74 \mathrm{~mm}$ in diameter, $152.40 \mathrm{~mm}$ (6 in.) in length, and $50.80 \mathrm{~mm}$ (2 in.) in gage section. Three LVDTs measure rod deflections at three adjacent points within the gage section to determine rod curvature, which is then correlated to the applied moment to characterize the 
mechanical properties of the bending rod. Online monitoring can capture mechanical property changes to reveal fatigue behavior during testing.
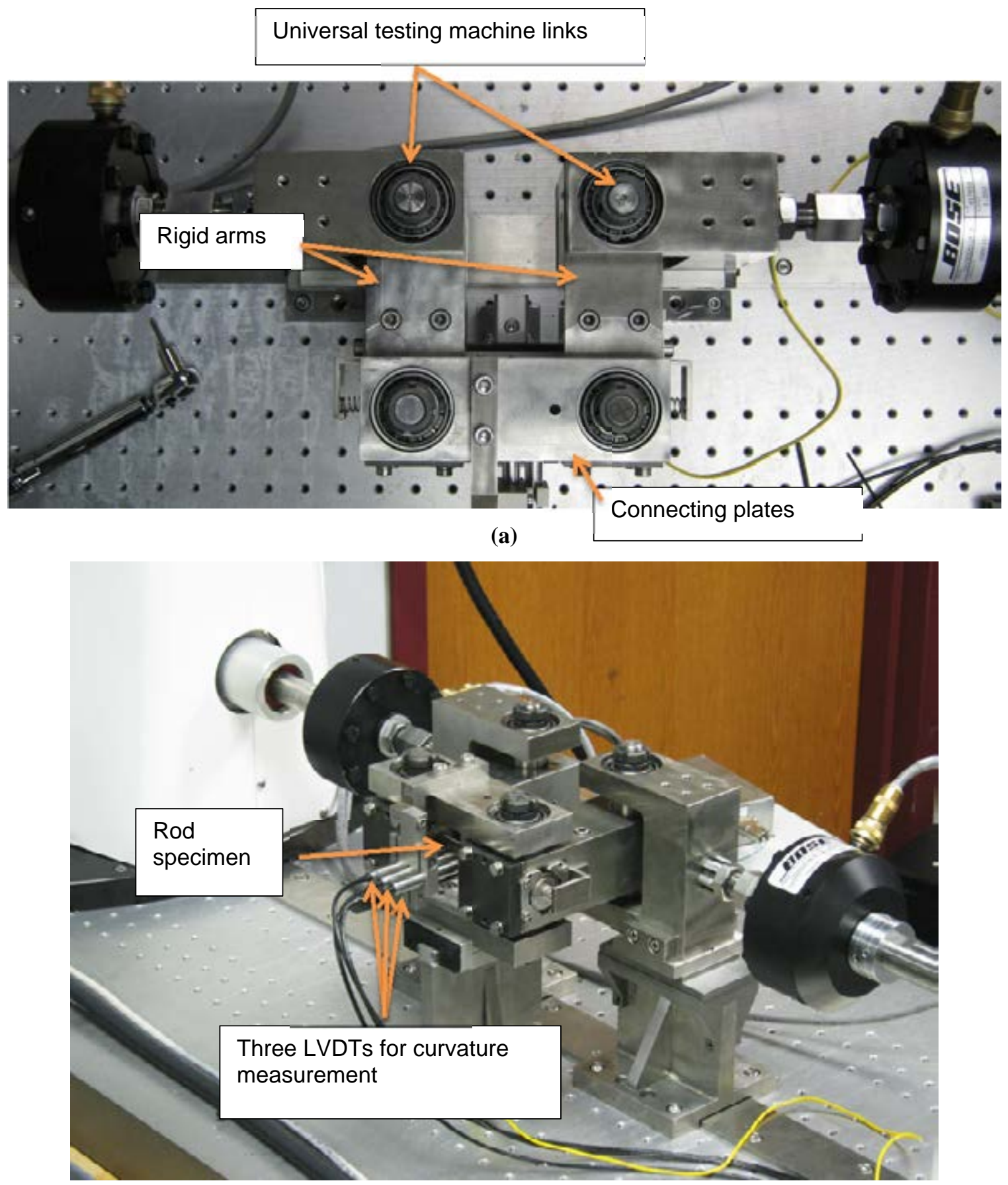

(b) 


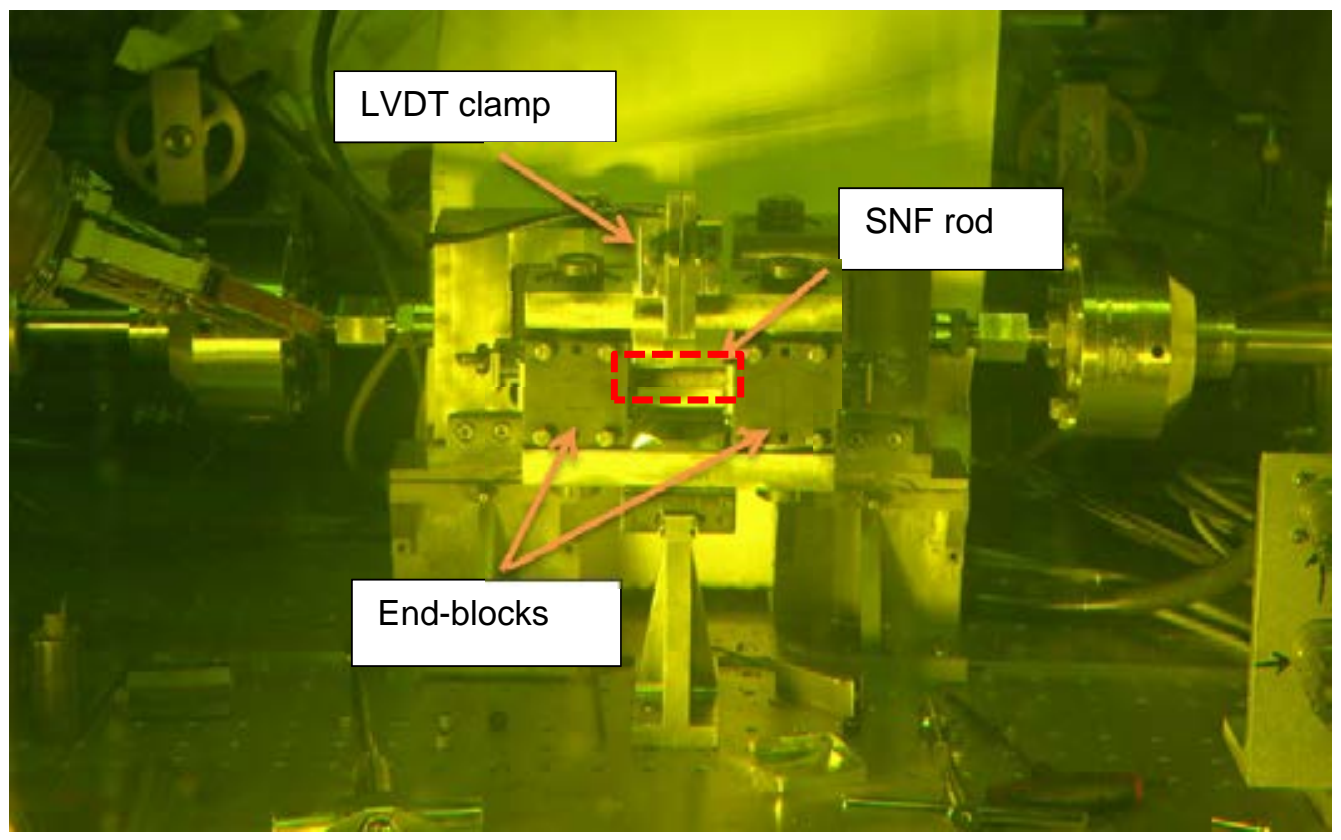

(c)

Figure 1. (a) Horizontal layout of ORNL U-frame setup; (b) rod specimen under test and three LVDTs for curvature measurement (operator is facing the three LVDTs); and (c) front view of CIRFT installed in ORNL hot cell.

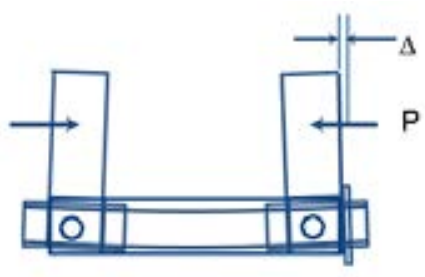

(a) Rigid arms are closing. The curvature is concave outward and designated with a negative sign.

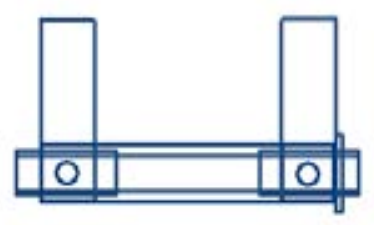

(b) Rigid arms are in neutral position.

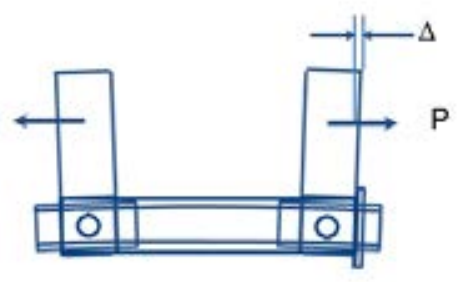

(c) Rigid arms are opening. The curvature concave inward and designated with a positive sign.

Figure 2. Schematic drawings of U-frame setup for reversal bending when rigid arms are (a) closing, (b) neutral, and (c) opening. 


\subsection{GRIP DESIGN}

Each grip consists of a $\mathrm{V}$-shaped block integrated into the rigid arm of the $\mathrm{U}$-frame, and a V-shaped end-block (Figure 3). The surfaces of the blocks serve as contacts to the specimen assembly and have been equipped with linear roller bearing sets embedded to ensure the free axial movement of the specimen when the force is being transferred. The rod specimen is secured by opening and closing the end-blocks in two grips. ${ }^{4,5,6,7}$ A partly exposed view on one end of the specimen is shown in Figure 4 with the end-block removed. The specimen has a pair of rigid sleeves on both holding areas. The sleeves protect the specimen from any contact damage through a compliant layer between the specimen and sleeves. The rigid sleeves also enable the specimen to move freely in the axial direction while being set against the embedded linear roller bearing sets. The free axial movement of the specimen is a critical requirement in pure bending, as discussed elsewhere in the literature. ${ }^{3}$

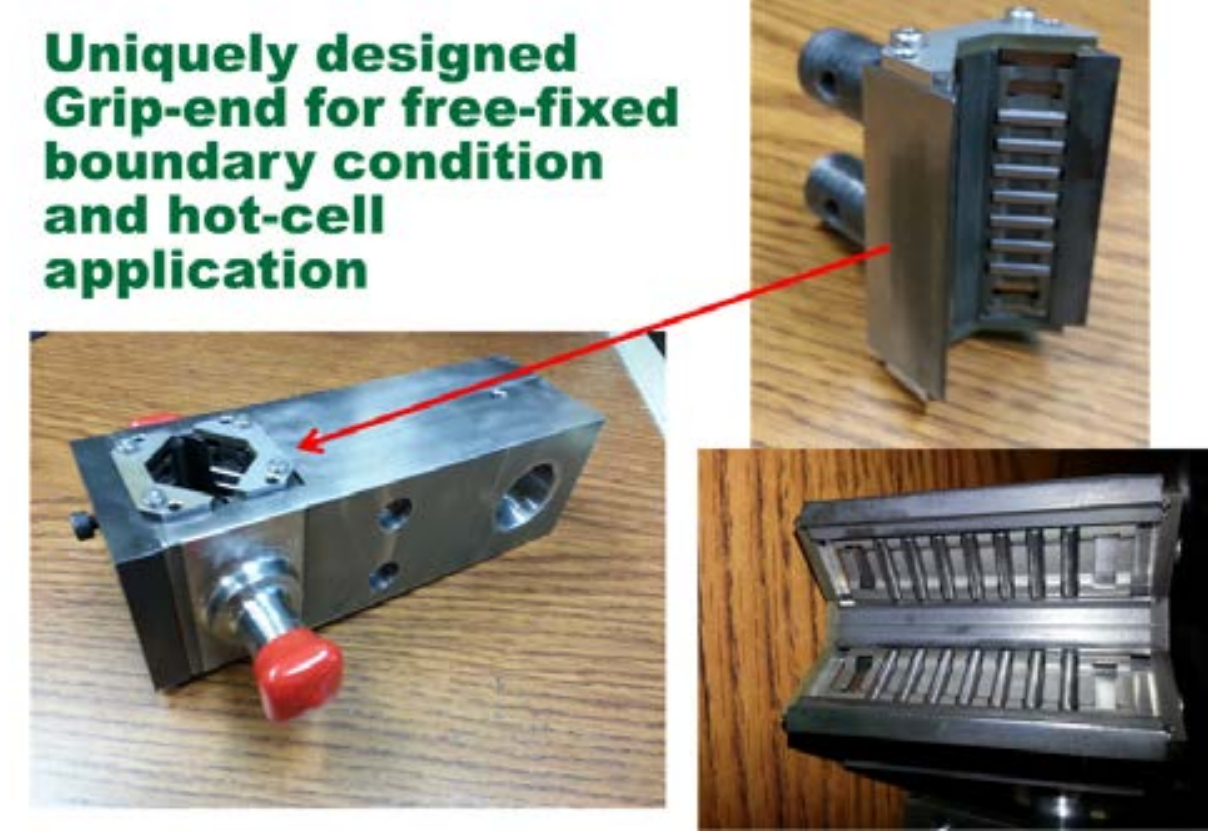

Figure 3. Assembled grip (left) and two views (right) of detached grip body. 


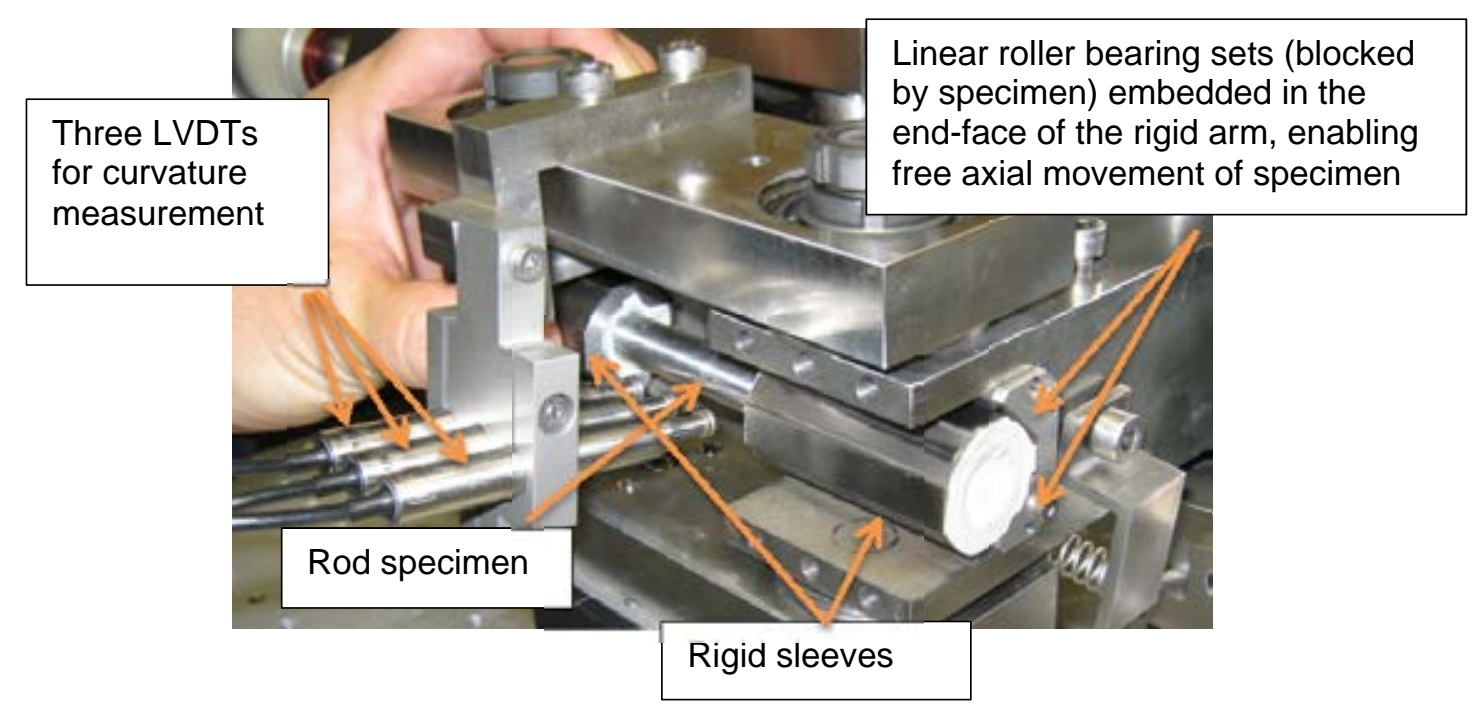

Figure 4. Image showing the grip design of CIRFT with one end-block removed.

The mounting of rigid sleeves onto the rod is achieved using casting epoxy. The cast epoxy, therefore, serves as the compliant layer whose size depends on the dimensions of the rigid sleeve and rod. The current rigid sleeves have an inside diameter (ID) of $15 \mathrm{~mm}$, an outside diameter (OD) of $25 \mathrm{~mm}$, and a length of $50 \mathrm{~mm}$. For fuel rods with an OD of $10.70 \mathrm{~mm}$, taken from the H. B. Robinson Nuclear Plant (HBR) reactor in South Carolina, the cast epoxy layer would have a thickness of $2.15 \mathrm{~mm}$ and the same length of rigid sleeves. Related physical and mechanical properties of the cast epoxy are listed in Table 1, along with those of the stainless steel in the U-frame setup. The use of cast epoxy as a compliant layer has been demonstrated to be effective in meeting requirements for both specimen protection and pure bending boundary conditions. ${ }^{4,7}$

Table 1. Physical and mechanical properties of related materials

\begin{tabular}{|l|l|l|l|l|}
\hline \multicolumn{1}{|c|}{ Materials } & Density $\left(\mathbf{k g} / \mathbf{m}^{\mathbf{3}}\right)$ & $\begin{array}{c}\text { Young's modulus } \\
(\mathbf{G P a})\end{array}$ & Poisson's ratio & $\begin{array}{c}\mathbf{0 . 2 \%} \text { yield strength } \\
\text { (MPa) }\end{array}$ \\
\hline Stainless steel & 8,030 & 193 & 0.30 & 290 \\
\hline Epoxy & 1,200 & 3.5 & 0.25 & 45 \\
\hline
\end{tabular}

Casting of the epoxy has been proven feasible in the hot cell environment. A vise mold has been designed and built for that purpose, and the procedures to cast the epoxy and mount the rigid sleeves into the rod specimen have been developed. ${ }^{4,5}$ Several important modifications were made to adapt the vise mold to hot cell testing. ${ }^{9}$ The images in Figure 5 (a) show different views of the vise mold, and those in Figures 5 (b) and 5 (c) illustrate the process of epoxy casting in the hot cell. The robust mold design enables two important functionalities for casting the CIRFT specimen in a hot cell environment: (1) the rod ends up in the center every time, and (2) the compliant layer is applied the same way each time so as not to introduce a variable.

The amount of fresh epoxy for each rigid sleeve is determined as needed in the hot cell based on the results from an out-of-cell study. In the case of the HBR rods, 5 grams ( $g$ ) of epoxy can fill the gap properly. The rod released from the vise mold is generally examined visually, and the gap will be refilled if a substantial cavity is observed between the rod and the rigid sleeves. 


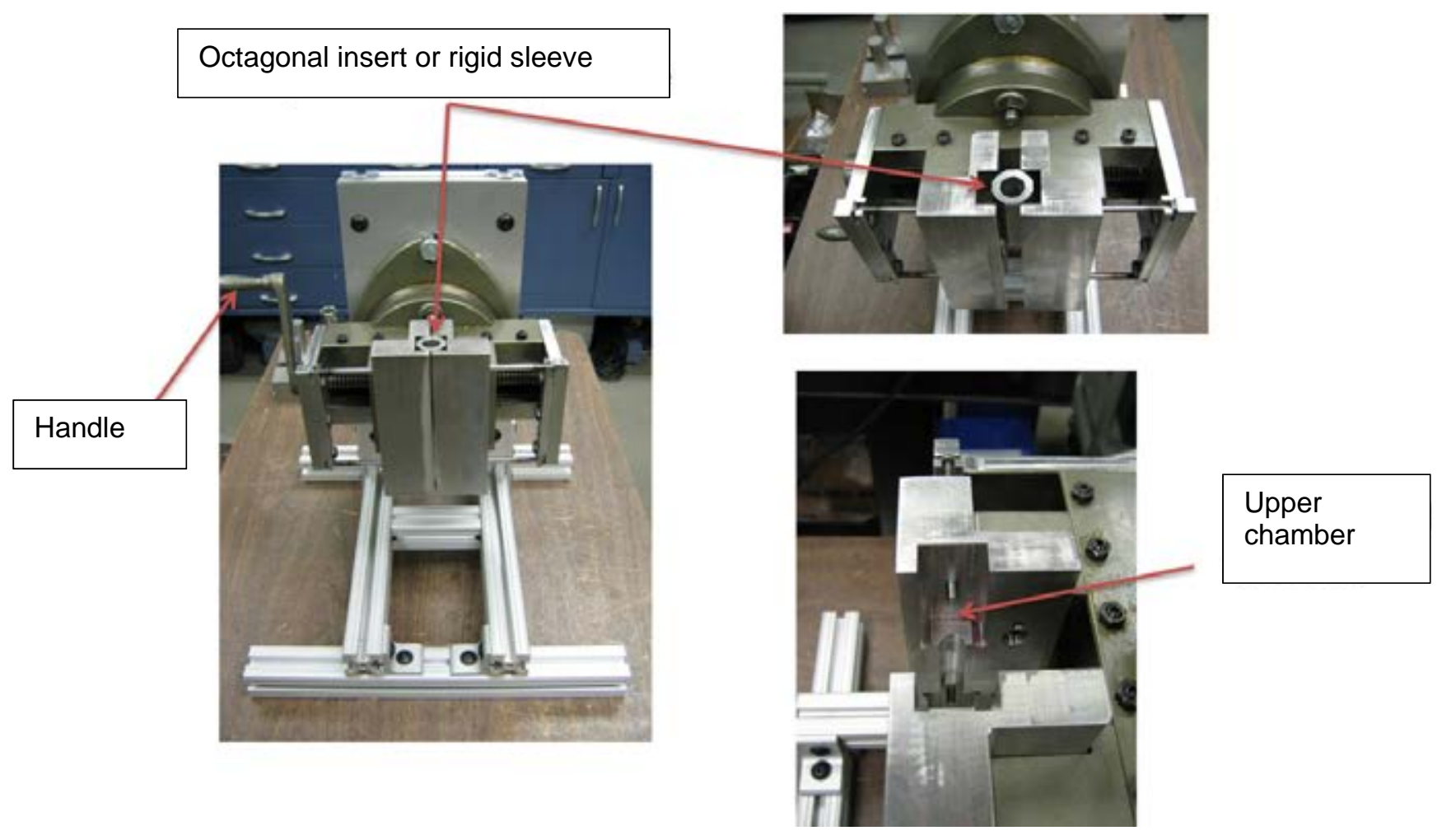

(a)

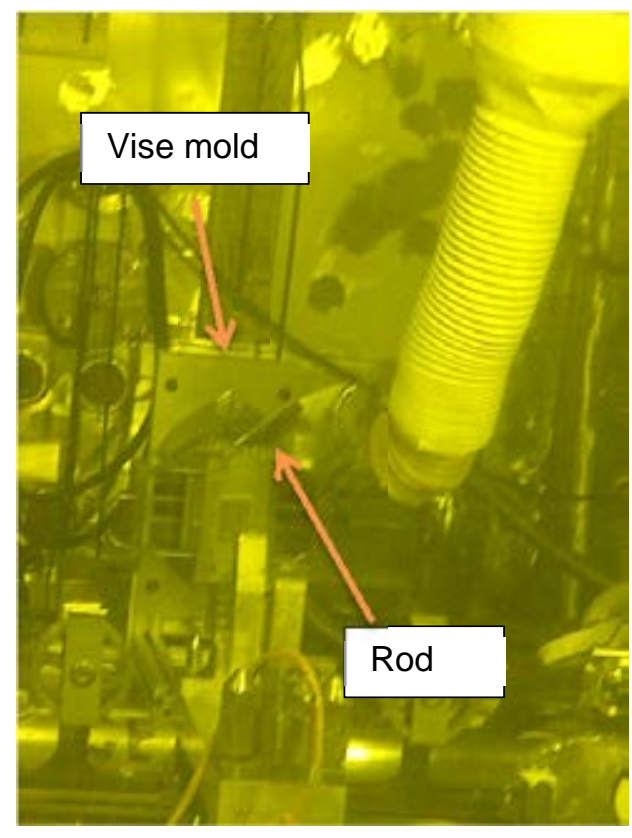

(b)

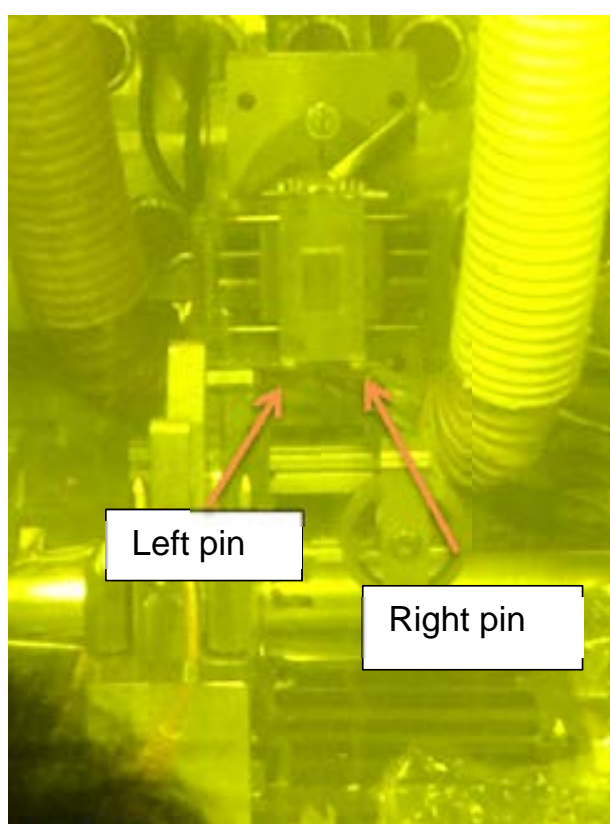

(c)

Figure 5. (a) Vise mold for assembling octagonal rigid sleeves into a rod; (b) rod (Demo1, 606B2) to be inserted; (c) one pin was turned away and a rigid sleeve loaded with epoxy inserted into the lower chamber. 


\subsection{LVDT MEASUREMENTS AND CURVATURE CALCULATION}

Theoretically, the bending radius and maximum strain of a rod can be estimated on the basis of the traveling displacement at the loading points of the rigid arm. The displacement measured, however, contains the contribution of the compliant layers, depending on the materials used in the compliant layers and the level of loading.

To address this issue, direct measurement of the specimen displacement at three adjacent points along the rod method was proposed ${ }^{12}$ and has been implemented to evaluate the curvature of a bending rod in this study. ${ }^{4,7}$

Given the deflections from three LVDTs, $d_{1}, d_{2}$, and $d_{3}$, as shown in Figure 6 , the curvature $\kappa$ of the bending rod can be evaluated as follows:

$$
\begin{aligned}
& \kappa=\left[\left(x_{0}-d_{2}\right)^{2}+y_{0}^{2}\right]^{-1 / 2}, \\
& x_{0}=\frac{-2 m_{a} m_{b} h-m_{a}\left(d_{2}+d_{3}\right)+m_{b}\left(d_{1}+d_{2}\right)}{2\left(m_{b}-m_{a}\right)}, \\
& y_{0}=-\frac{1}{m_{a}}\left(x_{0}-\frac{d_{1}+d_{2}}{2}\right)-\frac{h}{2}, \\
& \text { where } \\
& m_{a}=\frac{h}{d_{2}-d_{1}}, \\
& m_{b}=\frac{h}{d_{3}-d_{2}},
\end{aligned}
$$

and $h$ is the sensor distance, $12 \mathrm{~mm}$.

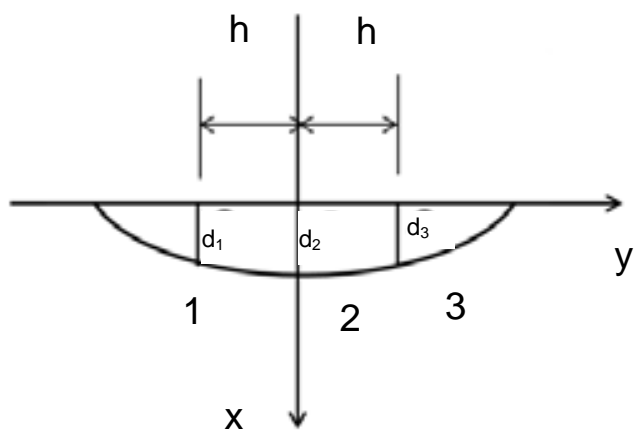

Figure 6. Determination of the bending curvature of the rod by use of deflections measured at three points.

The arrangement of the three LVDTs and their installation in the setup can be seen in Figure 1 and Figure 4. A series of static tests was performed to confirm the measurement method by using a surrogate rod composed of various materials. The curvature obtained by the LVDT measurements has been shown to correlate well with the calculated flexural rigidity of the specimen based on the estimated moment of inertia and Young's modulus for various materials. $^{7}$ 
The effect of contact between the LVDT probe and the rod on the curvature measurement became a concern, especially when loading during the hot-cell testing approached very small levels. The current LVDTs use flat, disk-shaped probes. The sensor spacing $h$ in the curvature calculation would be affected by the contact position of the probes. As shown in the upper view of Figure 7 (a), the contacts will be shifted outward and the $h$ increases correspondingly when the rod is concave toward the sensors; in the lower view, the $h$ decreases when the rod is concave away from the sensors.

In an out-of-cell study, the effect of the probe on the curvature measurement in dynamic testing has been investigated by using LVDT probes with a chisel contact. ${ }^{13}$ The results showed that the effect of probe geometry on the curvature measurement is negligible in dynamic testing within a low load range. Thus, the initial data reduction for the hot-cell testing did not envision the correction needed to account for the effect of the probe's width. However, CIRFT testing in the hot cell reveals the nonsymmetry curvature measurements for the clad tension and clad compressive loading cycles. This is partly because the SNF rod has a very limited pellet-pellet interface bond as compared to that of epoxied surrogate rod with well-bonded pellet-pellet interface structure. The potential sensor probe contact also has some effect. Thus, the SNF pellet-pellet debond interface-induced stress concentration effect cannot be readily observed or revealed from a surrogated rod tested under low loading amplitude. In order to further clarify the potential sensitivity of curvature measurement with regard to LVDT probe contact, a control experiment with a uniform polycarbonate surrogate rod was conducted, and the test results are illustrated in Figs. 7 (b) and (c). The test results of LVDTs located at the clad compression site show that the measurement with the disk probe overestimates the curvature by $50 \%$ compared to measurements with the chisel head probe. An adjustment factor of $\Delta \mathrm{h}=2.9 \mathrm{~mm}$ was applied to develop consistent results. For LVDTs located at the clad tensile site, the disk probe underestimates curvature by $40 \%$, and a correction factor of $\Delta \mathrm{h}=2.4 \mathrm{~mm}$ is needed.

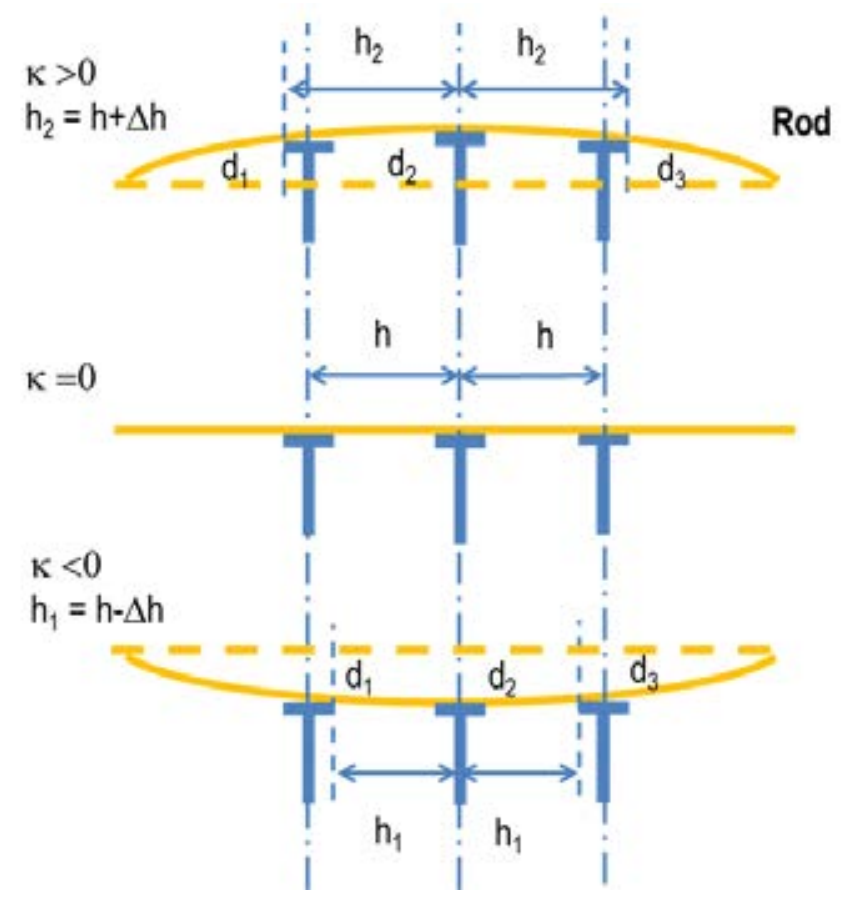

(a) 

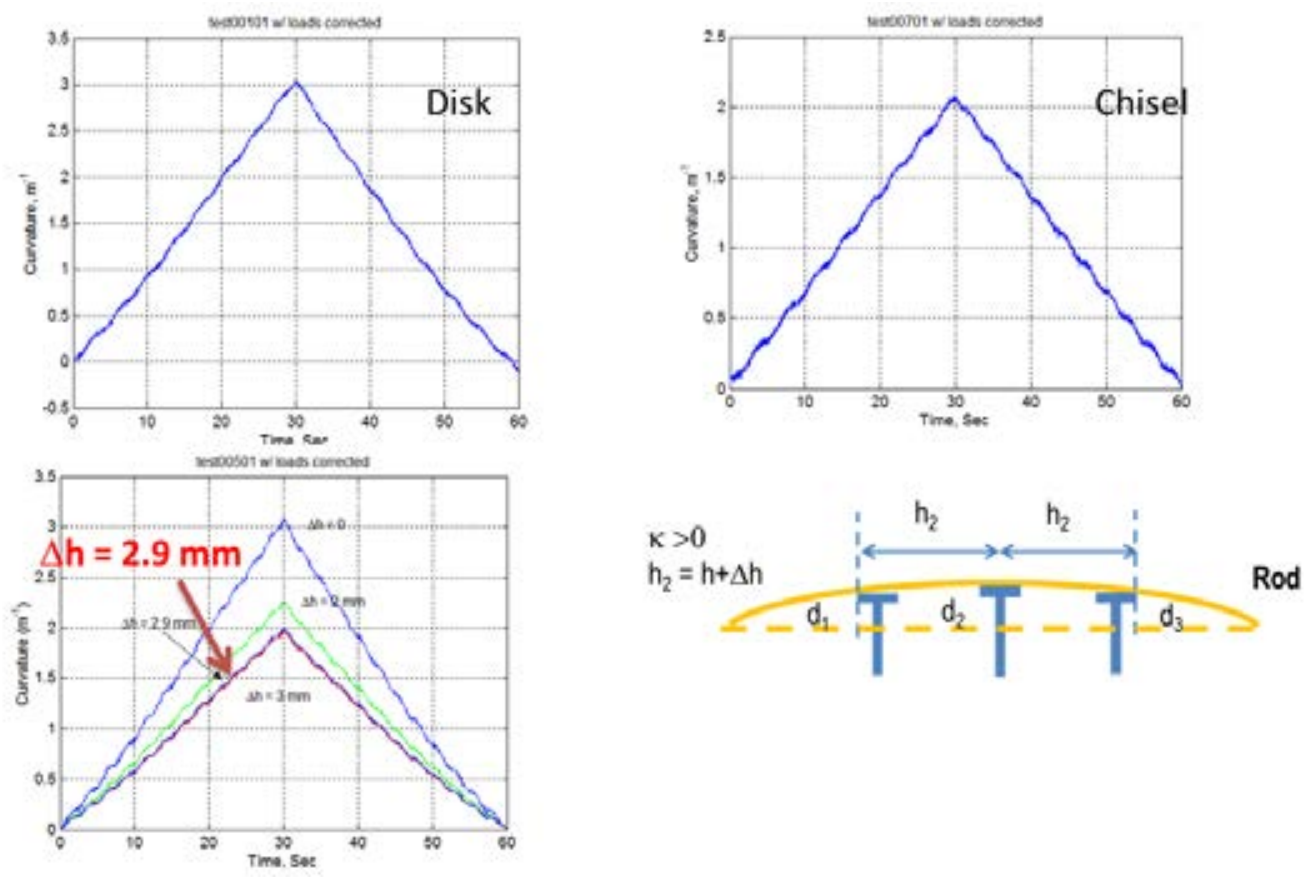

(b)
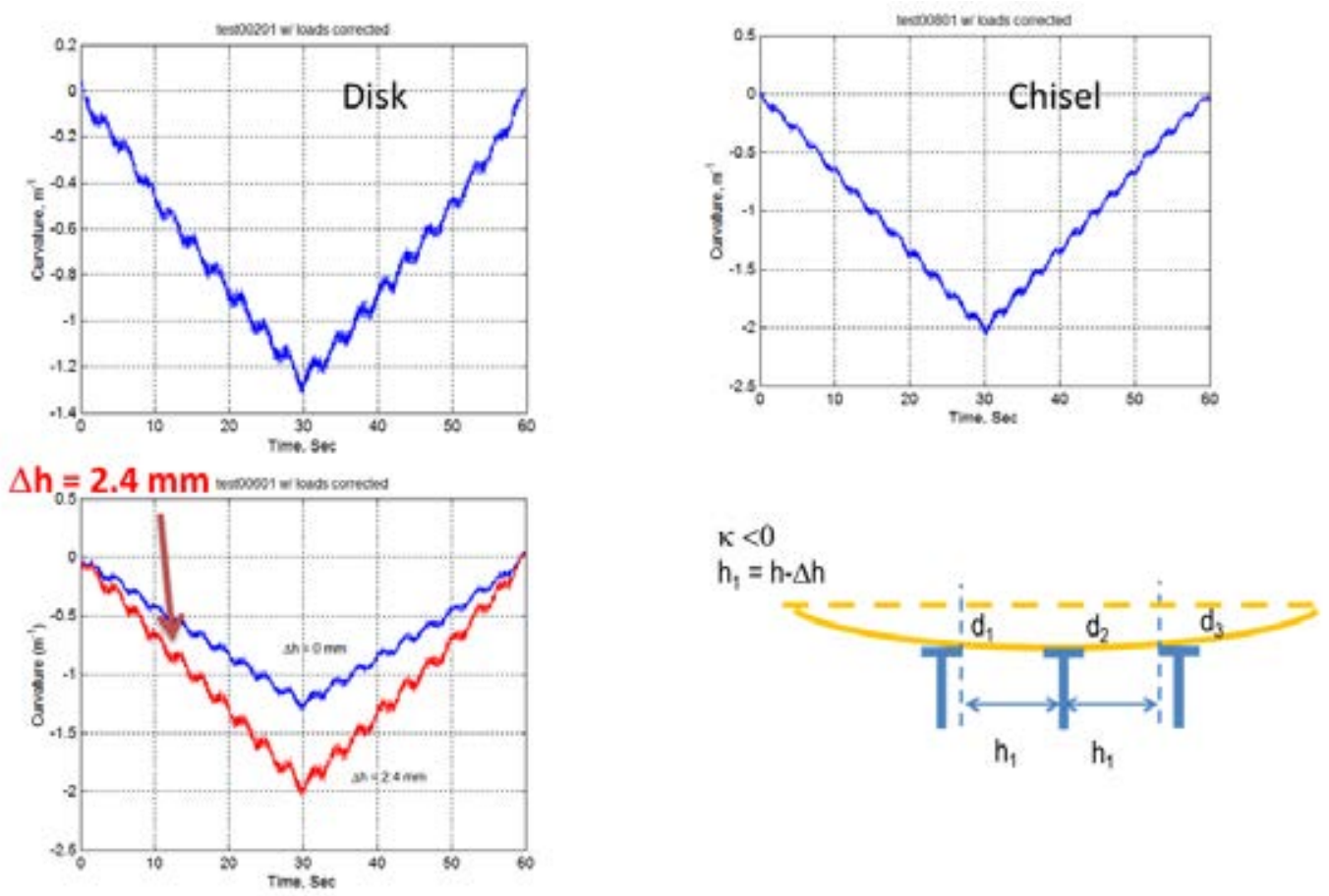

(c)

Figure 7. (a) Diagrams showing the effect of the LVDT probe with disk shape on curvature measurement, where the contact points are shifted. Comparisons of curvature measurement between disk and chisel head probe for LVDTs located at clad compression and tension site are shown in (b) and (c), respectively. (c). 
The adjustment factors presented above are only applied to a uniform rod system. Further investigation of using the surrogate rod with the debond pellet-pellet interfaces of a nonuniform rod system is needed to take into account the stress concentration effect and the increased curvature resulting from segment pellet structure. This research will help to finalize adjustment factor development.

\subsection{TEST SEGMENTS}

The Zry-4 $15 \times 15$ cladding (1.41 wt. \% Sn, $0.22 \mathrm{Fe}, 0.12 \mathrm{Cr}$, and $0.14 \mathrm{O}$ ) tested in this study was irradiated for seven cycles in the HBR Unit 2 PWR. The rod-average fuel burnup was 67 GWd/MTU. The test segments were taken from rods at locations E04, E14, F07, G10 and R05 in assembly $\mathrm{S}-15 \mathrm{H}$. The irradiation history of these rods and details can be found elsewhere. ${ }^{1}$

Although the goal of this project was to test static and cyclic bending performance, some rod segments were used for instrumentation demonstration, machine tuning, and benchmarking. The specimen cutting plan and the specimen locations in the father rods can be found in Appendix A.

A summary of specimen information for hot cell testing is provided in Table 2.

According to the plan, 6 in. segments were cut. Figure 8 shows one segment (608C4B) taken from the father rod $\mathrm{G} 10$, along with the tube used for specimen storage. The rod diameter of the fuel segments was measured using a dedicated setup in the hot cell. ${ }^{14}$ The measurement details are provided in Appendix $B$. Readings were taken at each half inch from one end of the rod and $3^{\circ}\left(0,120\right.$, and $\left.240^{\circ}\right)$ at each reading distance. The mean value and standard deviation of the diameter readings were found to be $10.772 \mathrm{~mm}$ (0.4241 in.) and $0.008 \mathrm{~mm}$ (0.0003 in.), respectively. It can be seen that the calculated mean value is close to the nominal diameter of the fuel cladding at HBR. ${ }^{1}$

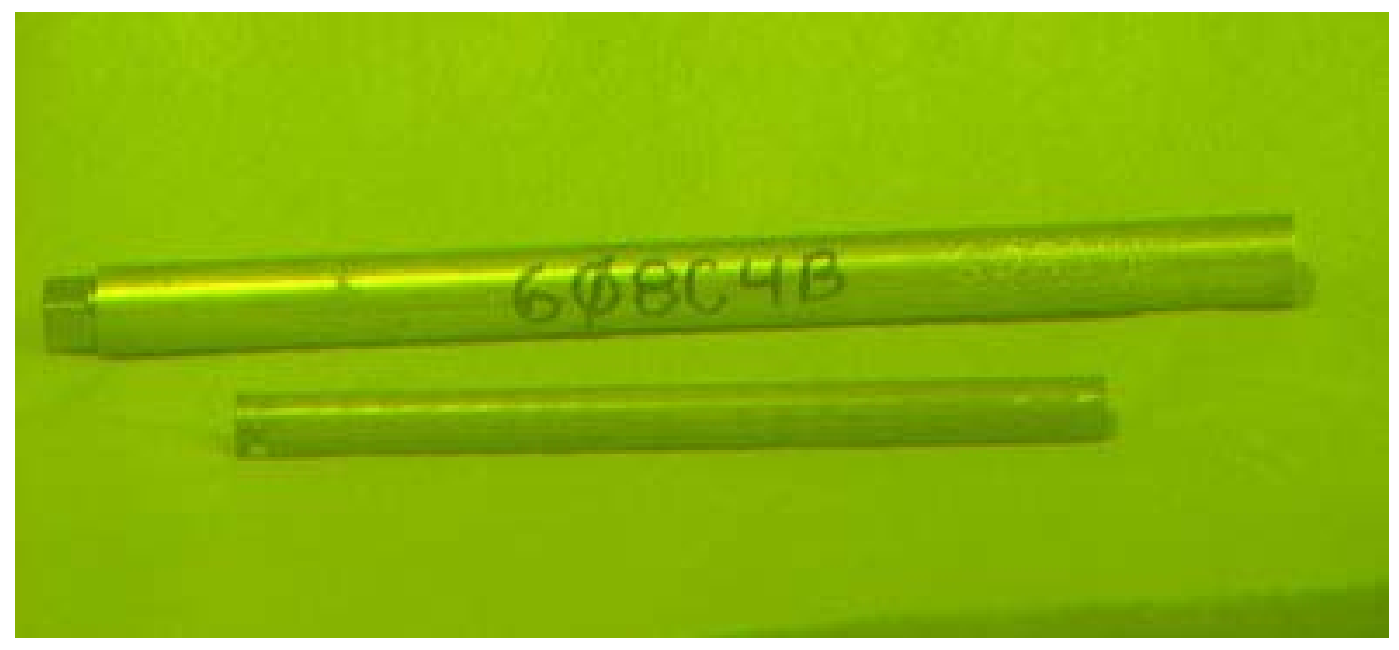

Figure 8. Segment 608C4B for DL2 taken from rod G10, with the storage tube. 


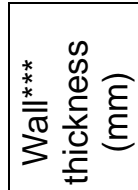

高
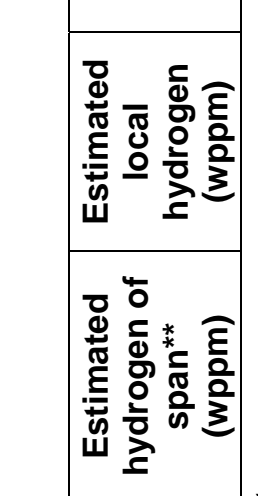

\begin{tabular}{l}
8 \\
\hline
\end{tabular}

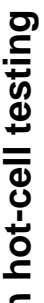

놀

营苋总 Ш

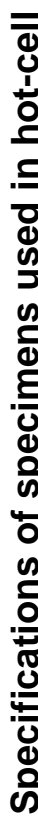

ำ

产旁

๙ัติ

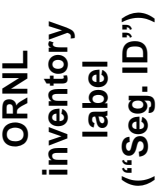

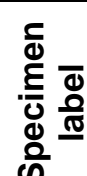

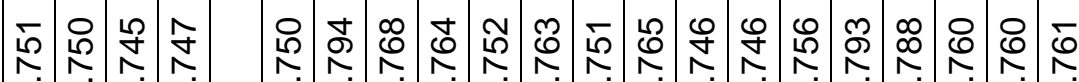

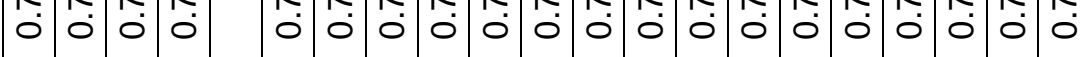

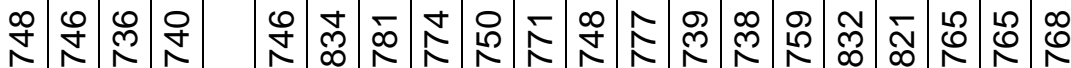

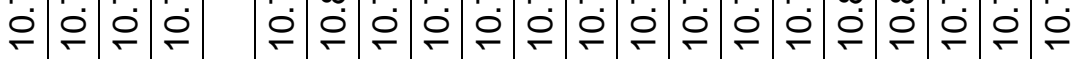

융 \&

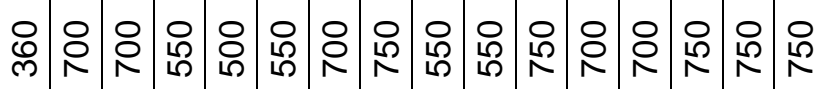

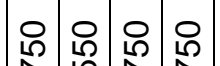

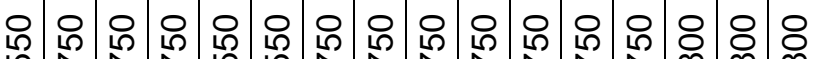

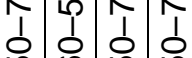

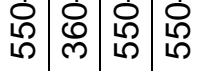

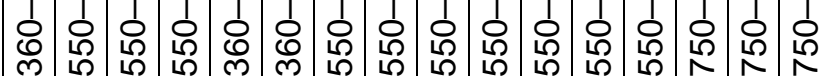
里

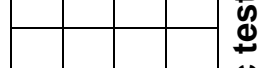

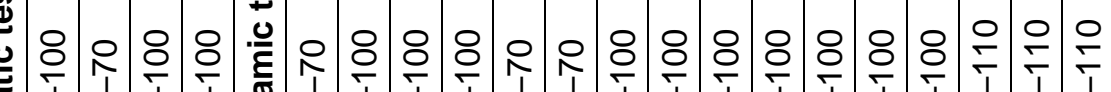
焉

\begin{tabular}{l|l|l|l|}
$\infty$ & 0 & 0 & 0 \\
0 & 0 & 0 & 0 \\
0 & 0 & $\varphi$ & 0
\end{tabular}

几

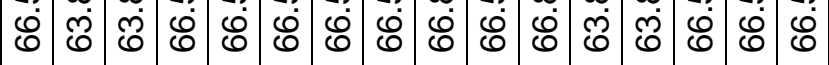

8

$\checkmark\left(\begin{array}{c}n \\ 1 \\ 1\end{array}\right) \forall$

N

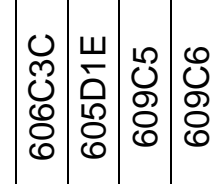
(1)

$\begin{array}{llll}1 & 1 & 1 & 1 \\ 0 & 0 & 0 & 0 \\ 0 & 0 & 0\end{array}$ -

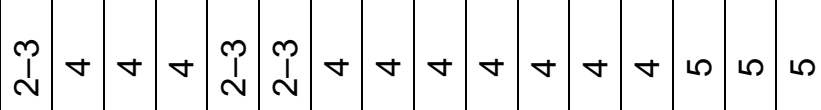

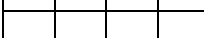

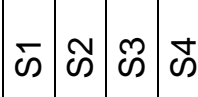

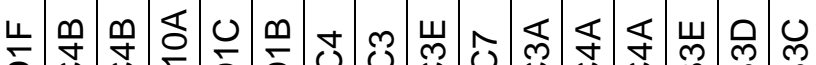

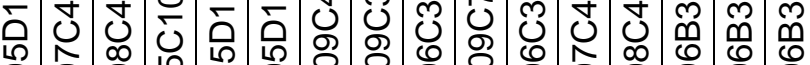

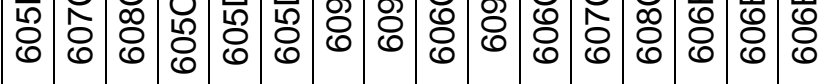
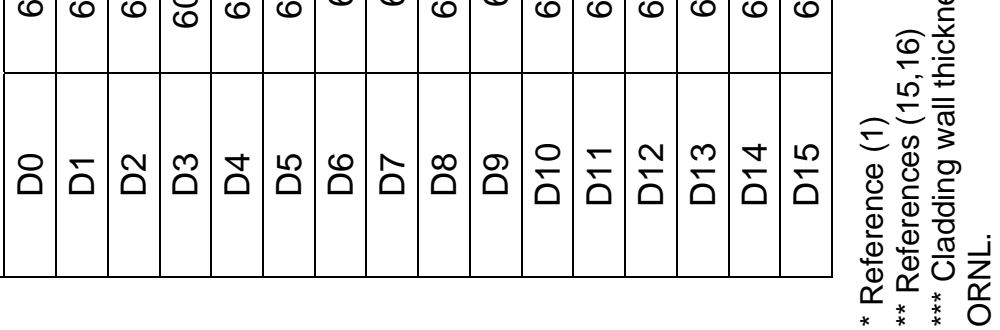


\subsection{TEST PROTOCOL}

The test system was calibrated under both static and dynamic testing conditions using three surrogate rods consisting of stainless steel cladding with alumina pellets. The system was tuned and benchmarked by testing a number of specimens. The related results can be found in Appendix C.

\subsubsection{Static Testing}

The purpose of static testing is to generate bending strength data for SNF rods. In particular, the static tests measure the bending strength of fueled SNF rods so that the contribution of the fuel to the SNF rod bending strength can be evaluated. The analysis of the static test results also provides a reference yield strength to establish the dynamic testing matrix. Static testing is carried out using displacement control. It involves ramping both loading arms of the U-frame at $0.1 \mathrm{~mm} / \mathrm{s}$ up to $12 \mathrm{~mm}$, where $12 \mathrm{~mm}$ displacement is the machine stroke capacity. The following procedure is followed.

- Perform the standard static bending test beyond SNF rod yielding to failure or up to device displacement or loading capacity.

- If the machine capacity is reached before specimen failure or specimen maximum strength is reached, repeat unidirectional static testing using the same condition for a few more loading/unloading cycles, or to specimen failure, whichever comes first.

- If the SNF rod does not fail after 3-4 loading/unloading cycles, follow a dynamic or cyclic test procedure to fracture the tested specimen to support postmortem examination. Such a dynamic test is called a follow-up test.

- Collect and weigh any fuel fragments that may have dislodged during the test.

The loading processes to be used after the first loading cycle were suggested because the device might not have sufficient stroke to test these particular specimens to failure.

\subsubsection{Dynamic Testing}

Dynamic testing consists of two major activities-dynamic real-time online monitoring and periodic quasi-static deformation measurements (see Figure 9). The procedure is as follows:

- Perform the dynamic cyclic test under constant load control using a sine wave input in reverse bending mode.

- Set the cycle frequency at $5 \mathrm{~Hz}$ and select the amplitudes for individual cycle tests considering the target cycles to be achieved with each test.

- Monitor the SNF fatigue evolution with defined intervals: perform static measurements of the rod deformation at the end of each target cycle with a frequency of $0.05 \mathrm{~Hz}$ and appropriate amplitude under displacement control.

- Stop the dynamic test when failure/clad fracture is detected or the preselected number of cycles is reached. 
- Weigh any fuel fragments that fall out of the fracture.

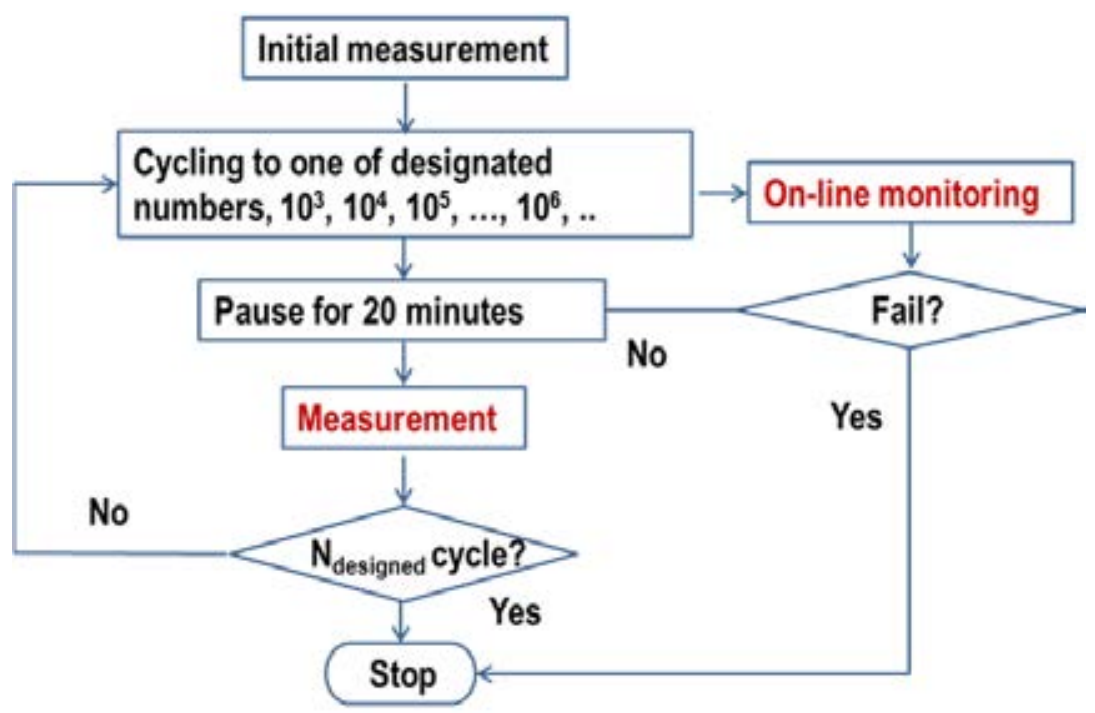

Figure 9. Flowchart for cyclic testing of spent fuel rod.

\subsection{DATA PROCESSING}

Measurement data and online monitoring data are converted into the applied moment and curvature, based on the load channel (load1 and load2) information, the loading arm length $(101.60 \mathrm{~mm})$, and LVDT data (LVDT1, 2, and 3). This information is used to generate the time series plots of moment and curvature and the moment-curvature hysteresis loops, such as those illustrated in Figure 10 for D1. The uniform moment applied to the rod is derived simply from the following equation.

$$
M=F \times L
$$

where $F$ is the averaged value of applied loads (load1 and load2) from the Bose dual motors, and $L$ is the loading arm length, $101.60 \mathrm{~mm}$. The computation of curvature $\kappa$ is described in Section 2.3.

An equivalent stress-strain curve can be obtained under the assumption that the SNF rod can be idealized as a linear elastic homogeneous material without consideration of the effects induced by pellet-cladding interaction. The equivalent stress was calculated using:

$$
\sigma=M \times y_{\max } / l
$$

where $I$ is the moment of inertia, $I=I_{c}+I_{p}, I_{c}$ and $I_{p}$ are moments of inertia of cladding and pellet, respectively, and $y_{\max }$ is the maximum distance to the neutral axis of the test rod section and is set equal to the radius of the cladding. The measured ODs of rod segments are given in Table 2. The calculation of stress disregards the difference of elastic moduli between cladding and pellets.

The equivalent strain is then:

$$
\varepsilon=\kappa \times y_{\operatorname{man}} .
$$




\subsubsection{HBU SNF Characteristics}

The SNF system contains multiphase components such as the cladding, pellets, and oxide. Thus, an SNF rod is far from an ideal homogeneous material, especially in HBU fuel, where the irradiated cladding and pellets and the associated interfaces are modified significantly during irradiation. Assuming the HBU rod has a perfect interface bond at pellet-cladding and pelletpellet interfaces, the associated flexural rigidity $E I$ can be expressed as

$$
E I=E_{c} l_{c}+E_{p} l_{p},
$$

where $I_{c}$ and $I_{p}$ are moments of inertia of the cladding and pellet, respectively, and the value of $I_{p}$ is based on the reference. ${ }^{1} E_{c}$ and $E_{p}$ are the Young's modulus of the cladding and pellet. The associated stress and strain evaluations of Eqs. (3) and (4) can still be used.

However, because of the imperfect interface cohesive bonding, the effective $E I$ will be less than that of the perfect bonding property, which can be written as

$$
E I=E_{c} I_{c}+E_{p} I_{p}-F(B E, \text { cladding, fuel }),
$$

where $F$ is the correction factor, which depends on interface bonding efficiency $(B E)$ and the fuel pellet and cladding aging properties, as well as cyclic loading amplitudes. This hypothesis was further validated in Reference 17.
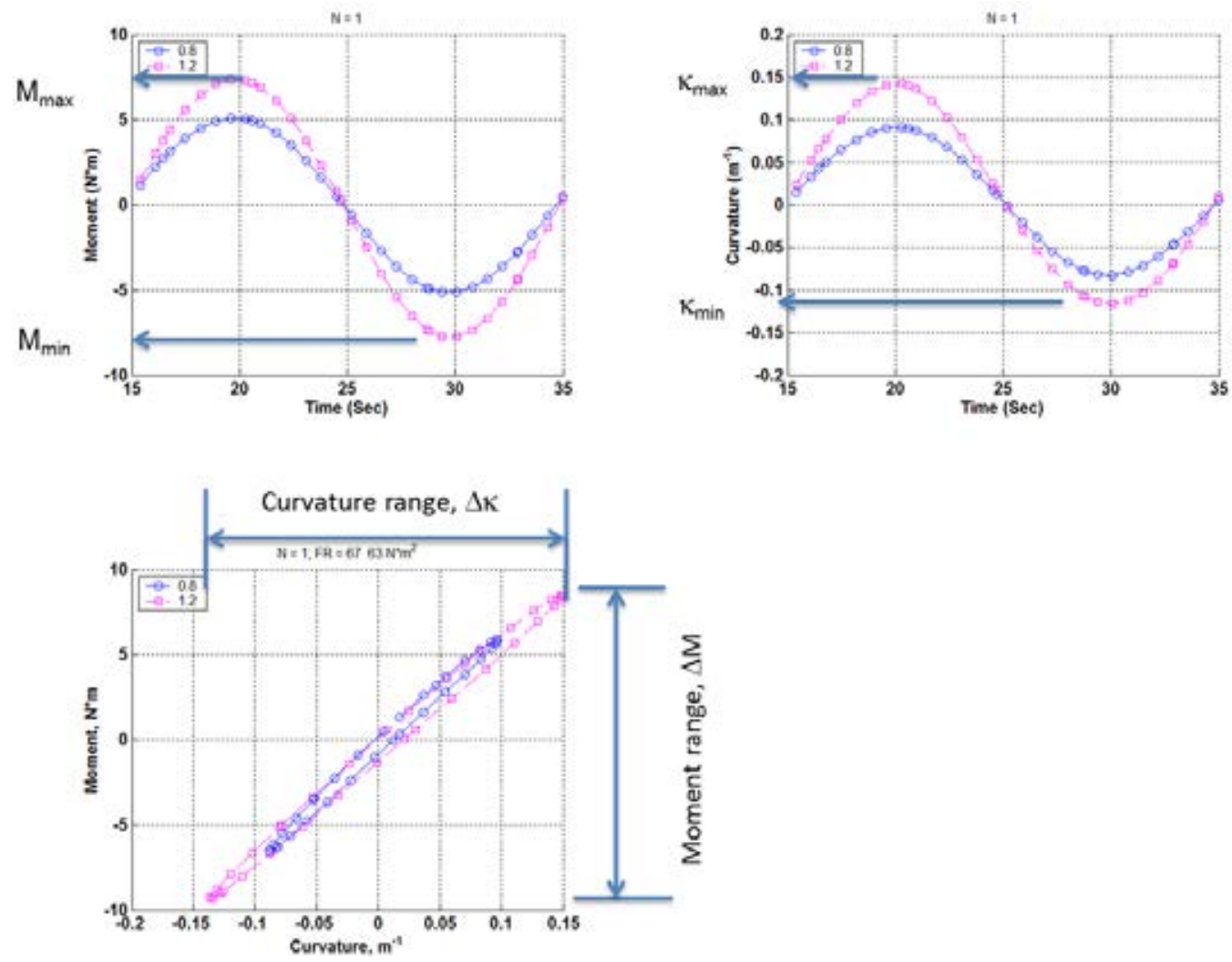

Figure 10. Moment, curvature time series, and moment-curvature hysteresis loop for rod D1. Two measurements are displayed with relative displacement 0.8 and $1.2 \mathrm{~mm}$ at the loading points of U-frame. 
Based on the static measurements of CIRFT testing, the El data trend indicates that the cohesive bonding at the pellet-pellet interface is fairly weak. The resulting degradation of flexural rigidity, which is associated with the debonding at the pellet-pellet interface, is reflected by the LVDT measured curvatures evidenced by a significant variation between the cladding tensile stress and cladding compressive stress regions shown in Fig. 10. This suggests that the fuel pellet reinforcement for SNF rod flexural deformation at the cladding tensile stress region is no longer available due to pellet-pellet debonding under flexural tensile loading. At the compressive cladding stress region, the fuel reinforcement remains intact in the form of the fuel pellet pinning effect. This results in a shift of neutral axis in an SNF system under reversal bending.

The above mentioned phenomenon was also observed from the CIRFT test online monitoring data, where the tensile cladding stress stage shows a higher curvature reading than the compressive stress stage, as shown in Fig. 24 (d). The El values also change accordingly in each moment reversal cycle, as shown in moment-curvature plot of Fig. 10, where the El (the slope of moment-curvature) in the clad compression cycle is higher than that of clad tension cycle.

Furthermore, a detailed three-dimensional finite element analysis with a $6.25 \mathrm{~N} \cdot \mathrm{m}$ uniform moment also reveals that the localized stress in the cladding at the pellet-pellet-cladding interface region is about three to four times (depending on the interface cohesive bond parameters) ${ }^{17,20}$ the LVDT measurement for the tensile cladding stress region, and the associated localized stress is about 2.6 times the average tensile cladding stress away from the pellet-pellet interface region. Another complication is that the neutral axis of the SNF rod will no longer reside in the geometric center of the SNF system, and the El value will shift alternatively around the geometry center under cyclic loading reversals. ${ }^{17}$ Therefore, the conventional approach, as stated in Eq. (3) and (4) from a global M-k consideration, is no longer valid for describing the cladding failure mechanism associated with localized flexural rigidity degradation at the rod pellet-pellet interface. Conducting detailed localized stress-strain evaluation at the pellet-pellet interface translated from global measurements is beyond the current scope of the project. Therefore, only the mechanical response derived from direct measurements (the LVDTs) will be utilized in this study and discussion.

In order to generate the stress-strain relationship associated with M-k, a first order approximation approach, commonly used for an isotropic/homogeneous structure, was adopted to translate the global CIRFT M- $\kappa$ data into equivalent stress and strain data of cladding. Where the SNF rod's moment of inertia is written as $I=I_{c}+c \times I_{p}, I_{c}$ and $I_{p}$ are moments of inertia of cladding and pellet, respectively, and $c=E_{p} / E_{c}$ (because the baseline Young's modulus of the oxide fuel is twice that of the cladding, $c$ is likely greater than 1). In the proposed equivalent stress and strain approach, $c$ is set to 1 , which implies a $50 \%$ reduction in pellet Young's modulus, to take into account the radiation induced degradation of the HBU fuel pellet, where the pellets are assumed to be perfectly bonded with the cladding at the pellet-pellet interface. Furthermore, setting $c=1$ implies the same $E$ properties for the fuel and the cladding, which allows the use of Eq. (3) and (4) under the homogenous properties hypothesis for an equivalent stress and strain evaluation.

Generally, the moment range $(\Delta M)$, curvature range $(\Delta \kappa)$, and flexural rigidity $E I$ are used in characterizing the mechanical properties of the fuel rod. These are defined as:

$$
\begin{gathered}
\Delta M=M_{\max }-M_{\min }, \\
\Delta \kappa=\kappa_{\max }-\kappa_{\min },
\end{gathered}
$$




$$
E I=\Delta M / \Delta \kappa
$$

where the subscripts "max" and "min" represent the maximum and minimum waveforms (Figure 10).

Under a load-controlling mode, the curvature response of a rod is not necessarily symmetric in one cycle of loading as will be discussed in Section 4.1. The offset of the M- $\kappa$ loop on the $\kappa$ axis with respect to the origin can be described by a mean value of curvatures, $\kappa_{m}$ :

$$
\kappa_{m}=0.5 \times\left(\kappa_{\max }+\kappa_{\min }\right)
$$

and the maximum of absolute curvature extremes, $|\kappa|_{\max }$, is

$$
|\kappa|_{\max }=\max \left(\left|\kappa_{\max }\right|,\left|\kappa_{\min }\right|\right) \text {. }
$$

For a given specimen, the $|\kappa|_{\max }$ given by Eq. (9) corresponds to the curvature that creates the maximum tensile stress in the cladding.

The resistance force of the CIRFT system may be significant, depending on the amplitude of the rigid arm movement. The CIRFT system static resistance force was measured at different displacement levels without a specimen loaded. The net applied load at each displacement level was estimated by subtracting the measured resistance from the applied static test load, shown in Figure 11. The CIRFT system resistance was tested and evaluated by using empty runs in which the specimen was not loaded on testing machine. This is conducted in the specified period or whenever the calibration is needed.

The effect of CIRFT system resistance on the dynamic loading of the rod was also investigated by using a calibration rod in an out-of-cell study. It was shown that due to low loading amplitude the net effect of system resistance and dynamic inertia on the load applied to the rod is negligible within the tested range. Since the out of cell condition that was evaluated is equivalent to the condition used in hot cell cyclic testing, no correction procedure needs to be applied to the data acquired during dynamic tests. 

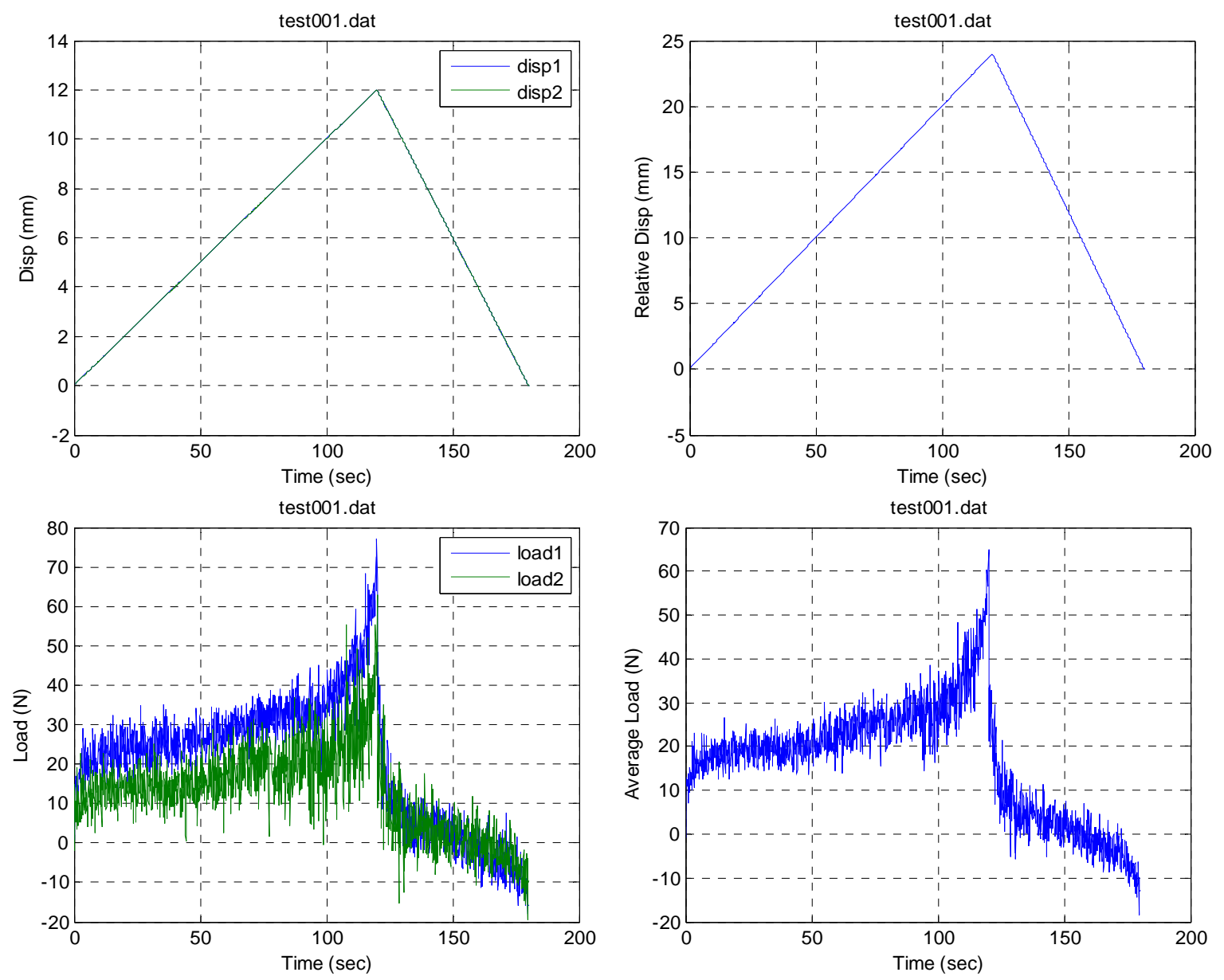

Figure 11. "Empty-run" conducted on HBR S1, for which the load and displacement were plotted. 



\section{STATIC TESTING}

\subsection{RESULTS}

Three specimens were tested under static loading in the hot cell for test system benchmarking. Four static tests were subsequently conducted. This chapter focuses on these four static tests; refer to Appendix $\mathrm{C}$ for the static benchmarking tests.

S1 (606C3C, $66.8 \mathrm{GWd} / \mathrm{MTU}$ burnup, 70-100 $\mu \mathrm{m}$ oxide layer) survived four static loading/unloading cycles of unidirectional bending without failure. The moment-curvature curve for the initial cycle is presented in Figure 12 (a). (The curves of subsequent cycles overlay one another to a great extent and for clarity are not plotted.) The maximum moment achieved in this test was $85.5 \mathrm{~N} \cdot \mathrm{m}$, corresponding to a maximum curvature of $3.2 \mathrm{~m}^{-1}$. The slope changes of the linear portion of moment-curvature curves could be seen approximately near moment levels 12 and $59 \mathrm{~N} \cdot \mathrm{m}$, respectively. It is noted here that due to pellet end dish-in design, the pellet-pellet interface bonding strength would be fairly weak, and the pellet-pellet de-bond phenomenon should be observed in the early phase of bending loading. The slope changed points indicate that the flexural rigidity changed in the SNF rod system within the linear elastic range. The changes of slope and associated deflection points are quantified in Section 3.2. The equivalent stress-strain curve based on Eqs. (3) and (4) for the initial cycle is presented in Figure 12 (b), where $Y_{\max }$ and I are based on the measured clad OD provided in Appendix B..

Since the machine capacity was reached and no failure occurred to the specimen, a follow-up dynamic test was conducted with reversible bending under \pm 25.40 to $30.48 \mathrm{~N} \cdot \mathrm{m}$ at $5 \mathrm{~Hz}$. As mentioned above, the purpose of this procedure was to test the specimen to failure for further postmortem examination. The rod failed after approximately $1.4 \times 10^{4}$ moment reversals. The failure occurred near motor 1 (right side) with a fuel release of 0.9 grams. Optical images of the fracture surfaces are shown in Figure 13. The pellet end-face can be identified from the haze zone characterized by a brown-blue color (indicated by the arrow in Figure 13a). This indicates that the rod failure may have initiated at the pellet-to-pellet interface. At the same time, a serrated shear fracture surface of the cladding was observed. The serrated fracture surface was believed to be the end stage of the crack propagation upon the fatigued CIRFT sample fractured. Note that this specimen appears to have been loaded into the test machine at $90^{\circ}$ from the specimen ID marks.

The cladding surface (Figure 13 [c] and [d]) near the neutral axis of the bending rod is free of visible surface oxide spalling, which is expected since these regions experienced little stress. In contrast, one of the stressed lateral surfaces which experienced the maximum flexural stress is covered with significant visible circumferential surface oxide spalling. 


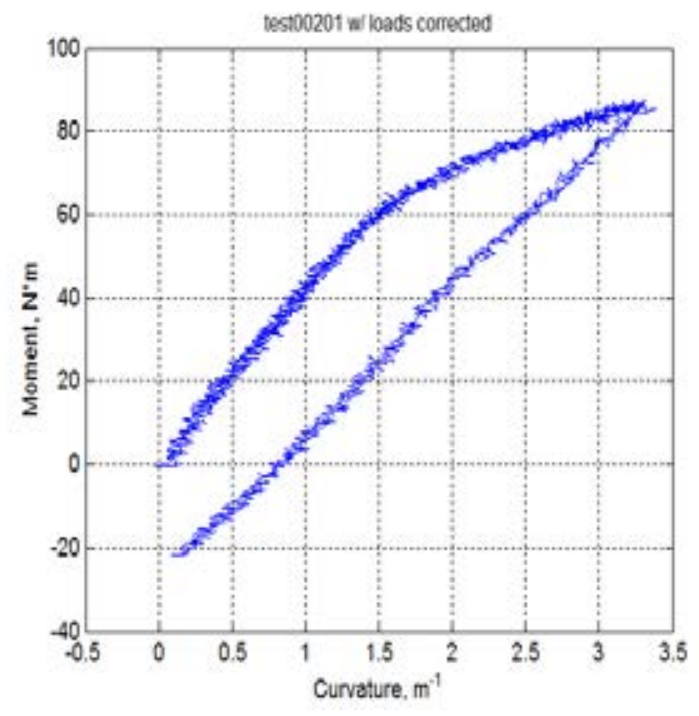

(a)

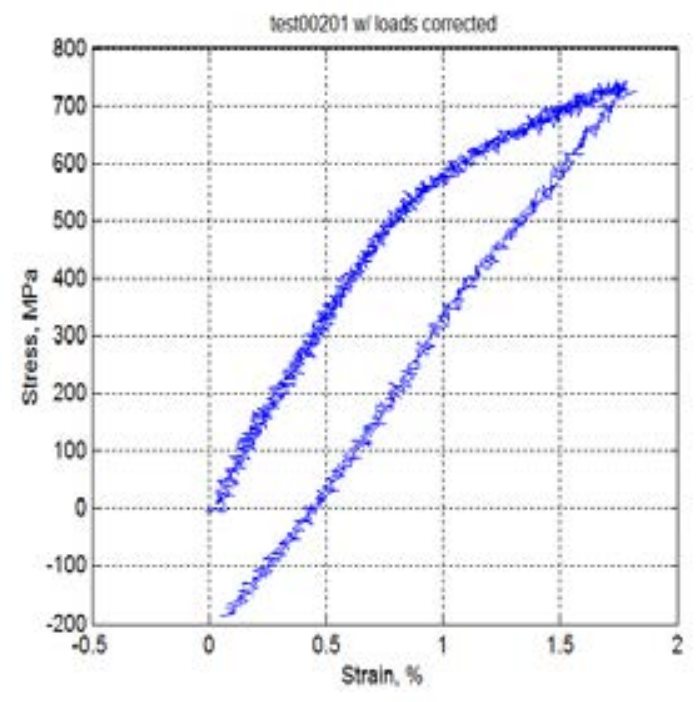

(b)

Figure 12. (a) Curve of moment versus curvature, and (b) equivalent strain-stress curve ${ }^{1}$ for S1 (606C3C). Based on a static loading cycle in which the maximum relative displacement was $24 \mathrm{~mm}$; rates at the loading points of the U-frame were set at 0.1 and $0.2 \mathrm{~mm} / \mathrm{s}$ for loading and unloading, respectively.

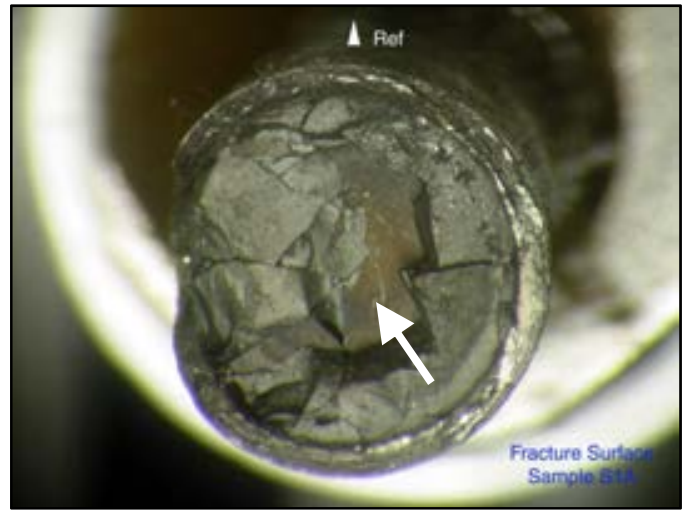

(a)

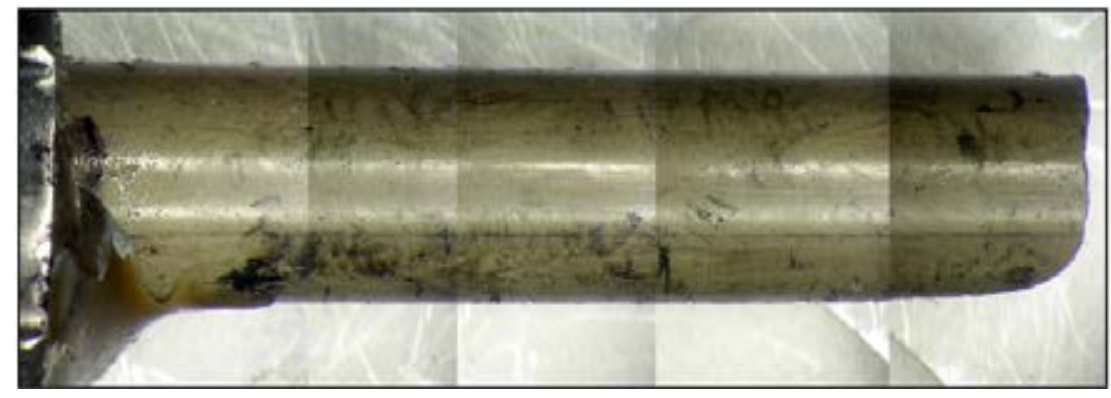

(c)

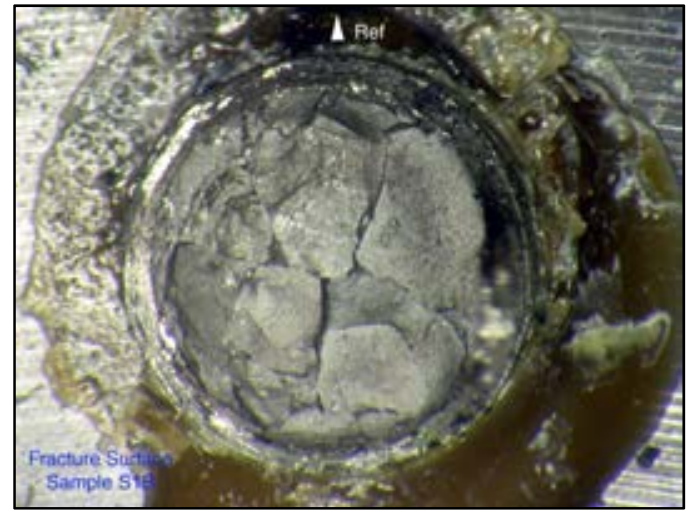

(b)

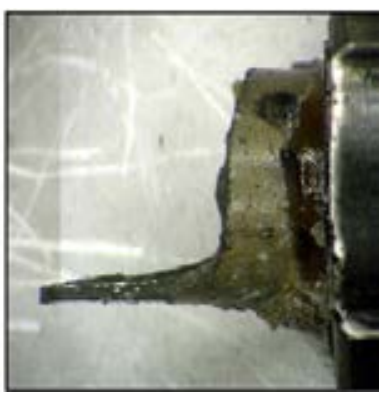

\footnotetext{
${ }^{1}$ Based on Eq. (3) and Eq. (4).
} 

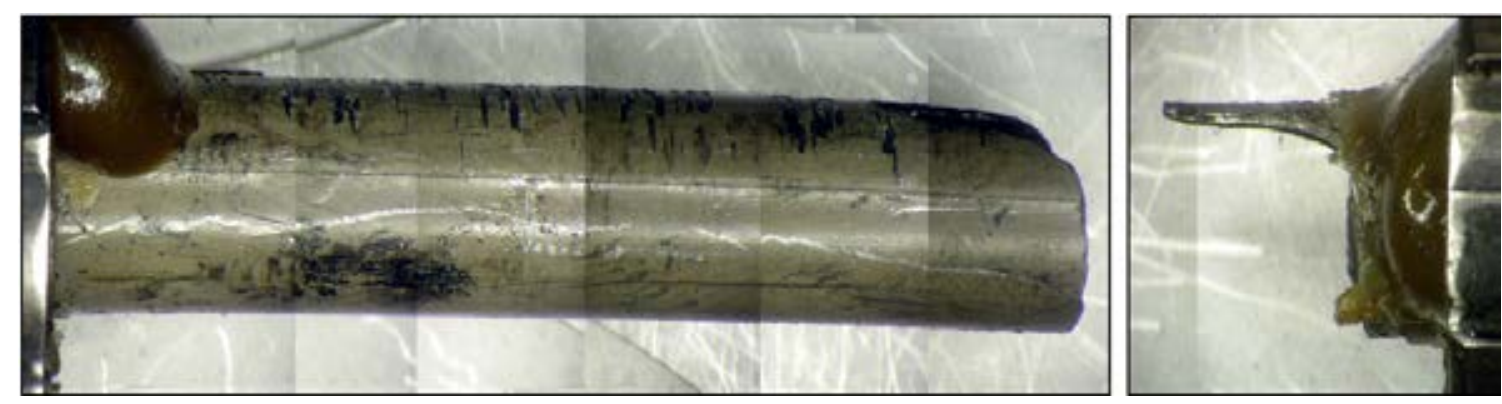

(d)

Figure 13. Mating fracture surfaces (a) and (b); lateral sides near the neutral axis of the bending rod for $\mathrm{S} 1(606 \mathrm{C} 3 \mathrm{C})(\mathrm{c})$ and (d). The rod was subjected to four repeat static cycles to $24 \mathrm{~mm}$ maximum relative displacement followed by dynamic cyclic loading \pm 25.40 to $30.48 \mathrm{~N} \cdot \mathrm{m}$ at $5 \mathrm{~Hz} ; 1.4 \times 10^{4}$ failure cycles, $0.9 \mathrm{gram}$ fuel particles collected. Note: This specimen appears to have been loaded into the test machine at $90^{\circ}$ off from the ID marks

Specimens S2 and S3 were both tested under static condition for three loading/unloading cycles without any sign of failure. They failed in follow-up dynamic tests under $\pm 30.48 \mathrm{~N} \cdot \mathrm{m}$ at $5 \mathrm{~Hz}$. The amplitude of applied moment in the dynamic tests was 35 to $36 \%$ of the maximum applied moments used in the respective unidirectional bending process, located in the early second stage of moment-curvature curves. It is noted here that the selection of the applied moment amplitudes was to generate the failure of the tested specimens within a reasonable number of cycles. The bending fatigue response of the as-received fuel rods will be discussed next. Finally, S4 was tested under static conditions for three cycles and failed during the fourth cycle. Details of these static tests can be found in Appendix D.

It should be noted that, in the static tests reported above, loading/unloading cycles did not result in apparent or catastrophic failure of specimens because of the limited stroke of the CIRFT machine. The failure of specimens occurred in the initial loading cycle of two benchmarking tests, Scal1 and Scal2. The peak moments of the two tests were 78.2 and $80.7 \mathrm{~N} \cdot \mathrm{m}$, respectively. Details of these benchmarking tests can be found in Appendix $\mathrm{C}$.

A summary of the moment-curvature curves is shown in Figure 14 for the four static tests. The top and right axes present the scale of the converted maximum strain and stress of the bending specimen according to Eqs. (3) and (4).

Several important observations can be made with respect to the responses of the test specimens during the first loading cycle.

- The static test results of the four specimens prior to the follow-up dynamic testing all show relatively similar elastic behavior.

- $\quad$ The initial moment-curvature response is characterized by a flexural rigidity of 52 to 63 $\mathrm{N} \cdot \mathrm{m}^{2}$.

- There is a change in the flexural rigidity at the moment of 12 to $21 \mathrm{~N} \cdot \mathrm{m}$. This change will be quantified in Section 3.2. 
- After this change, the moment-curvature response is characterized by a degraded flexural rigidity of 37 to $41 \mathrm{~N} \cdot \mathrm{m}^{2}$.

- Each static test segment experienced 1-2\% plastic strain without failure.

- The unloading flexural rigidity is similar to the loading flexural rigidity in the second stage after the moment reaches 12 to $21 \mathrm{~N} \cdot \mathrm{m}$.

Overall, the rods failed within the gage sections in all of the tested rods. The majority of the rod fractures involved the pellet-to-pellet interfaces (PPIs). S3 was exceptional in that the failure location seemed away from pellet-pellet interface, as no end face of the pellet can be identified from the fracture surface of the fuel. A summary of static test results is provided in Table 3.

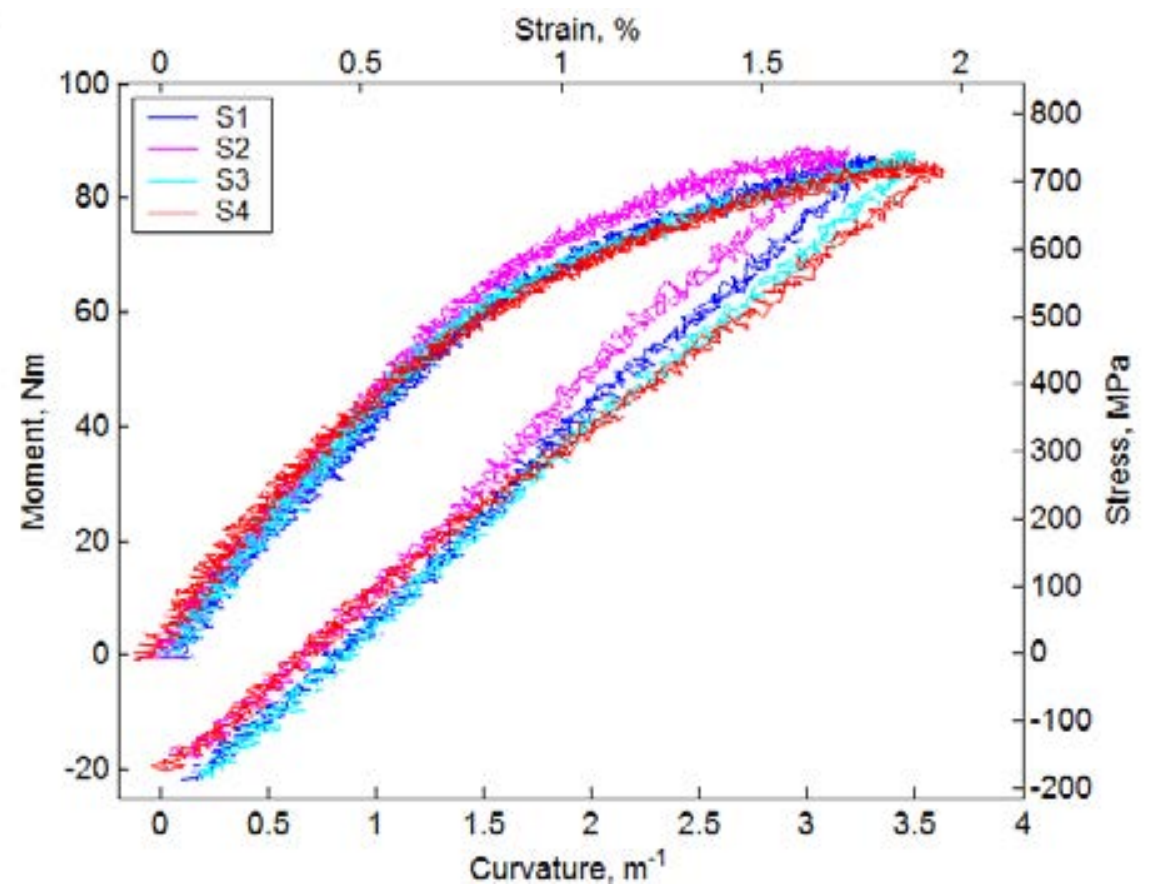

Figure 14. Moment-curvature curves measurements in static tests (Corresponding stress and strain displayed on right and top axes, respectively). 


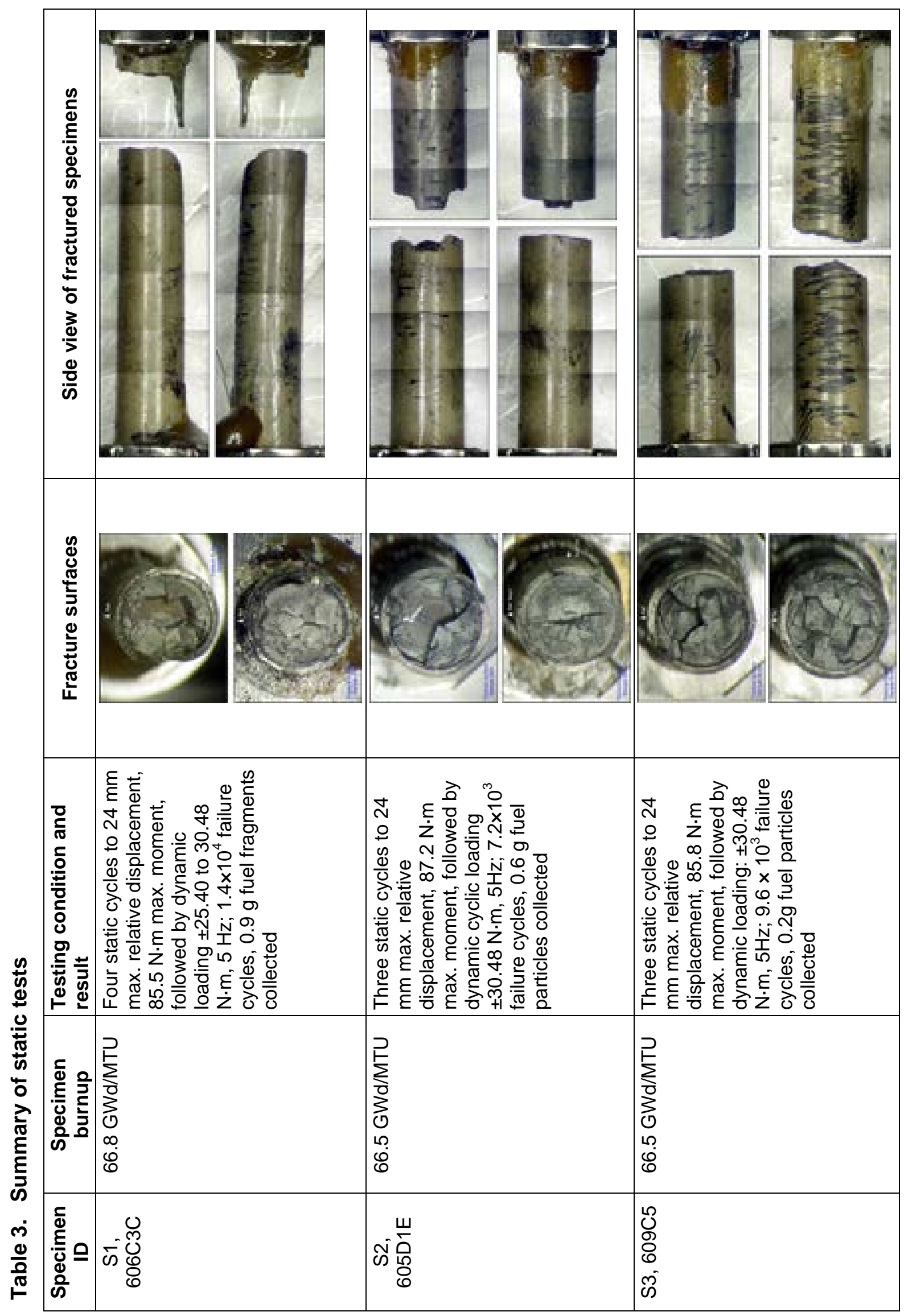




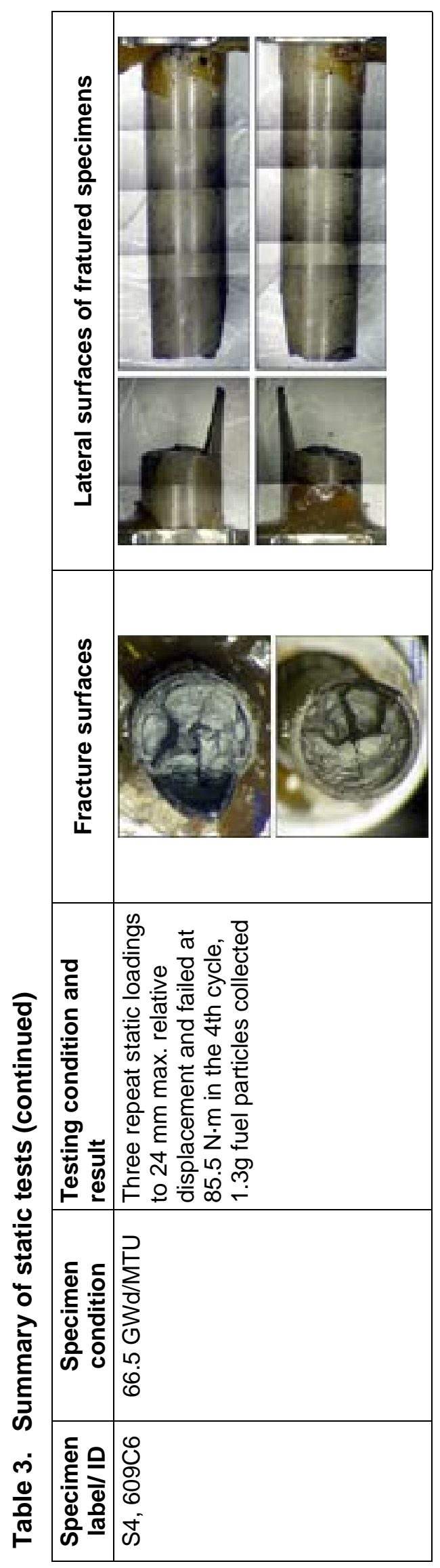




\subsection{DISCUSSION}

\subsubsection{Characteristics of Moment-Curvature Curve}

The moment-curvature responses of all four static test specimens were similar. They are characterized by two linear constituent behavior responses, followed by a nonlinear response during the loading and a linear response upon unloading. It was observed that when reloaded, the rod followed the unloading curve linearly and proceeded with nonlinear response after passing the previously achieved maximum load. Such loading/unloading/reloading responses can be found in many mechanical systems where strain hardening prevails. ${ }^{7}$

An effort was made to characterize the moment-curvature response based on the characteristic points to facilitate understanding test results. The flexural rigidities EI1, EI2, and EI3 were obtained, corresponding to the slopes of the first and second linear segments and of the unloading segment by using curve fitting with the first order polynomial (Figure 15). The characteristic curvatures and moments at the slopes' changed points A and B were then identified. In addition, the moment at point $C$ corresponding to a $0.37 \mathrm{~m}^{-1}$ irreversible curvature, or $0.2 \%$ equivalent plastic strain, was found by using a line with the same slope as that of unloading and horizontal axis intercept $0.37 \mathrm{~m}^{-1}$. The quantities corresponding to points $A, B$, and $C$ are designated by $\kappa_{A}, \kappa_{B}, \kappa_{C}$, and $M_{A}, M_{B}$, and $M_{C}$.

The results are summarized in Table 4. For a given specimen, EI1, EI2, and EI3 are generally in decreasing order with a marginal difference between the latter two. The characteristics derived from equivalent stress-strain curves are provided in Table 5. The E1 in the initial stage of the stress-strain curve was 83 to $101 \mathrm{GPa}$, and the $0.2 \%$ yield strength $\left(\sigma_{\mathrm{C}}\right)$ was 687 to $727 \mathrm{MPa}$. The ranges of the elastic modulus and the $0.2 \%$ yield strength appear consistent with the range of HBU HBR cladding. ${ }^{18}$ However, the observation should not be overemphasized, for the results observed here reflect a comprehensive global response of fuel rods with both pellets and cladding included. Moreover, substantial simplifications were made with regard to the elasticity and contributions of individual components, where the localized stress riser in cladding at the pellet-pellet interface region was not considered. 


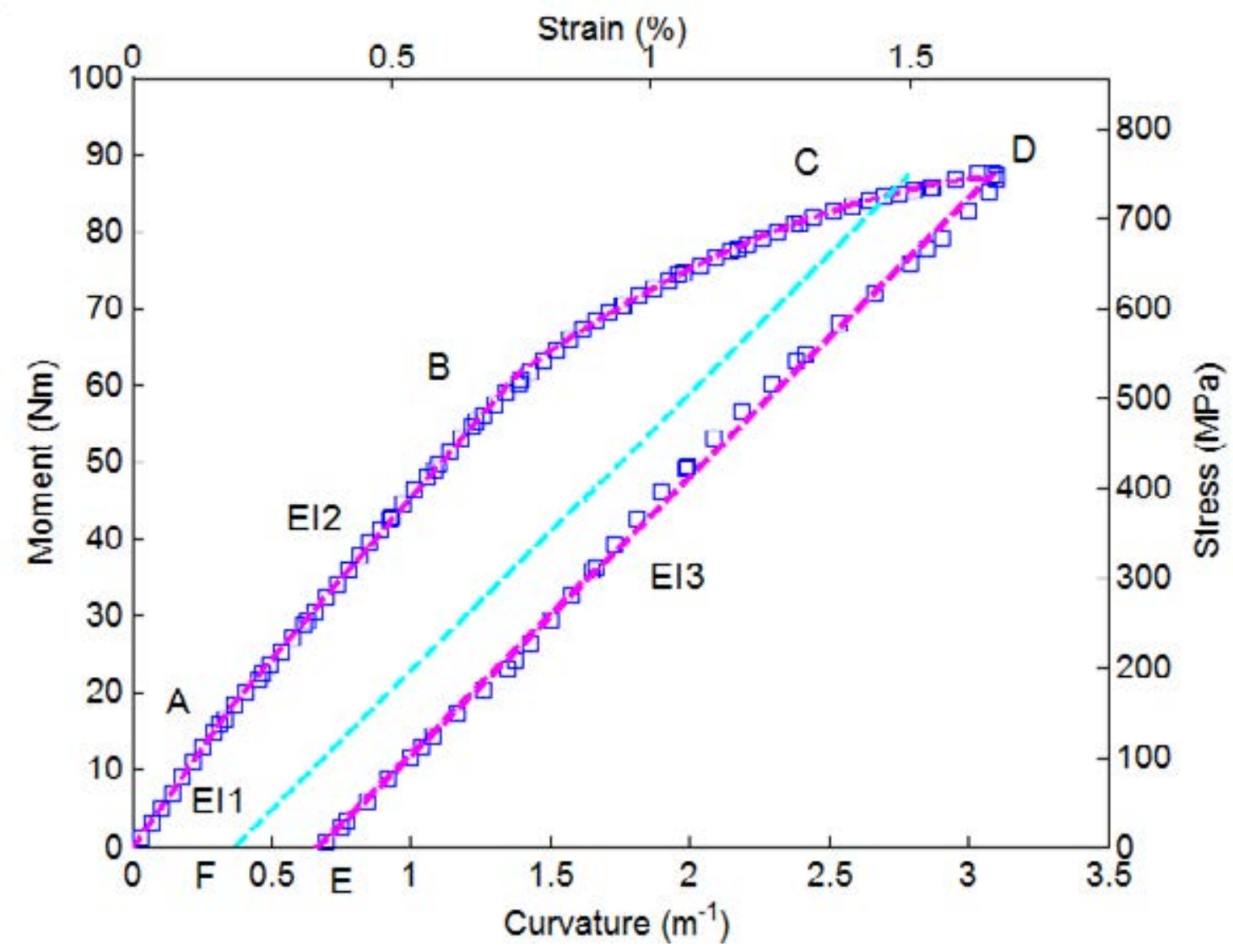

Figure 15. Characteristic points of moment-curvature curve. 

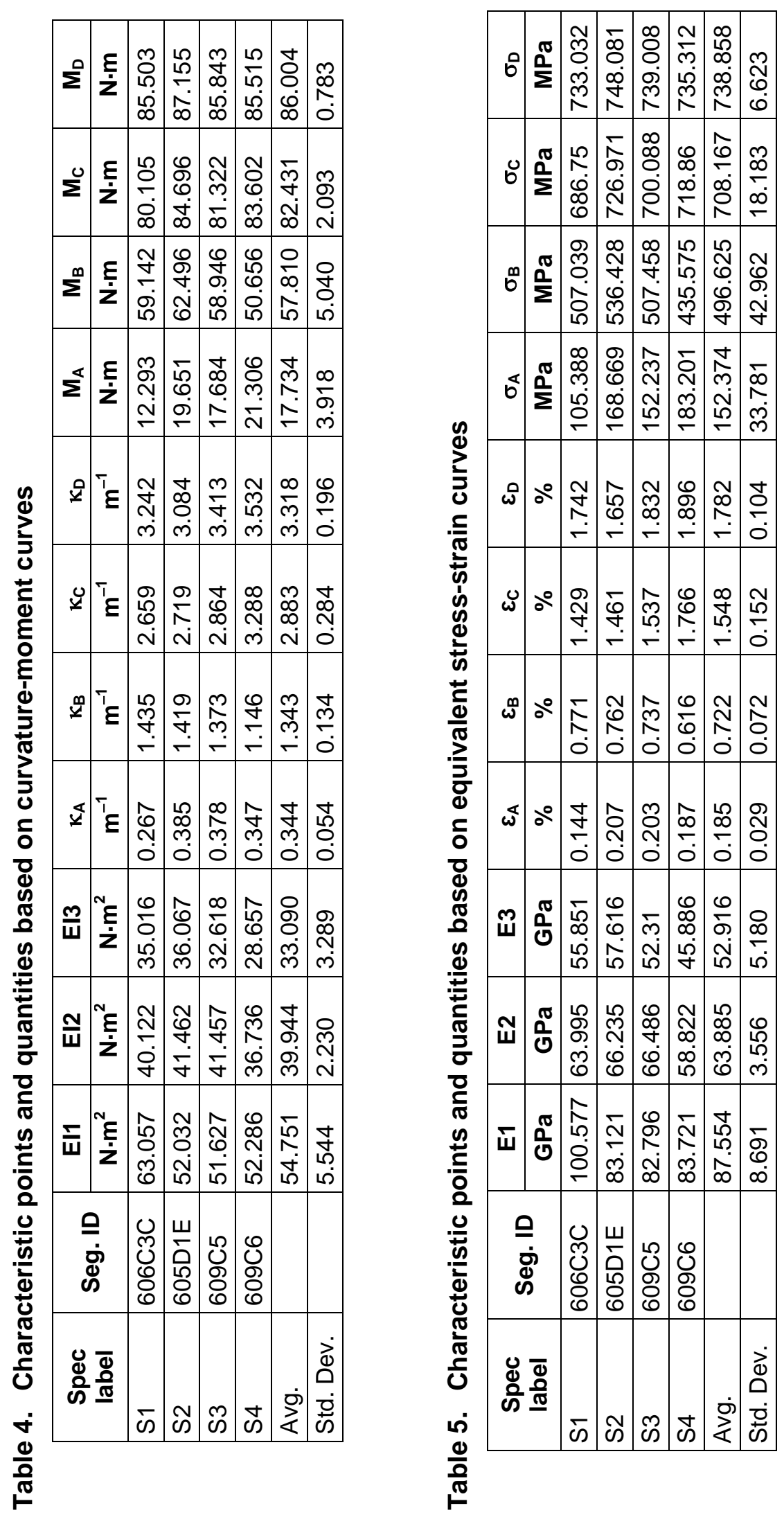


\subsubsection{Comparison of Static Results with PNNL Cladding Data}

In order to investigate the contribution of fuel pellets in the fuel rod structure, analysis was performed to compare the measured static results to predicted values considering cladding alone. The cladding properties used for this analysis were obtained from a database maintained by $\mathrm{PNNL}^{19}$. The database allows the user to specify the cladding type, temperature, fluence and cold work of the cladding of interest. For this study, the following values were specified:

Cladding - Zry-4

Temperature $-75^{\circ} \mathrm{F}$

Fluence $-1210^{25} \mathrm{n} / \mathrm{m}^{2}$

Cold work -0.5

Using these values in the PNNL database, the Young's modulus was given as $9.15 \times 10^{10} \mathrm{~Pa}$, the yield stress $919 \mathrm{MPa}$, the uniform elongation 0.00393, and ultimate tensile strength $976 \mathrm{MPa}$. From these values the engineering stress and strain values can be calculated.

It is noted that the CIRFT LVDTs measured the global rod deformation within the gage section. Thus, the stress-strain estimate is an average evaluation throughout the gage section that covers several pellet lengths with several pellet-pellet interfaces. For the linear response of the cladding region of the rod away from the pellet-pellet interface, evaluation of the stress and strain can be a straightforward composite flexural rigidity formulation due to relatively good bond at the pellet-cladding interface. However, due to the composite structure segmented pellets, the localized stress riser or curvature magnification at the pellet-pellet interface region cannot be estimated directly from the equivalent stress and strain approach; instead, they need to be evaluated using FEA ${ }^{17,19}$.

In order to further quantify the fuel pellet support or reinforcement to the SNF system mechanical property with respect to bending flexural response, the comparison based on CIRFT

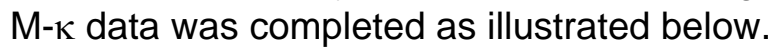

For comparison, the PNNL data ( $\sigma-\varepsilon$ curve) were converted to a moment-curvature curve by using the following equations, with the consideration of stress distribution that is appropriate for a thin-walled tube under uniform bending,

$$
\mathrm{M}=\sigma \cdot 1 / \mathrm{y}_{\max }
$$

and

$$
\kappa=\varepsilon / y_{\max }
$$

where $I=I_{c}$, and other quantities have same meanings as in Eq. (2) and (3). The hypothetical "cladding only" moment-curvature response was plotted together with the moment-curvature response of the high burnup fuel rod system as shown in Figure 16, where the SNF rod shows much higher bending moment resistance compared to that of PNNL data with cladding alone. A comparison of CIRFT testing results with cladding-only rigidity based on PNNL data is given in Error! Reference source not found.. HBR's rod used in the flexural rigidity calculation has the following cladding dimension: cladding ID 0.364", OD 0.423".

In the data comparison above, a distinct difference from that of the four HBU fuel rod systems was observed in the slope of the PNNL data. The slopes of the four HBU fuel rod systems are greater than that of the PNNL data. By focusing on the initial slope, between $0-15 N^{\star} m$, the slope (flexural rigidity) of HBU fuel rods was approximately twice that of the PNNL data for cladding alone. The increase in flexural rigidity can be attributed to the presence of fuel. Nevertheless, a detailed analysis is required to determine exactly how the presence of fuel 
accounts for this increase in flexural rigidity, as well as the net effect of fuel support on SNF rod system fatigue lifetime.

The increase of SNF rod system stiffness is attributed to the fuel's mechanical properties and fuel's geometry property of the moment inertia. However, the measured flexural rigidity of a fuel rod system is much less than that estimated from a direct summation of $E_{c} l_{c}+E_{p} l_{p}$. This is primarily due to interface bonding de-efficiency at fuel-cladding and fuel pellet-pellet interfaces, as well as existing fuel-clad gap potential. All these will affect the degree to which the presence of fuel results in increased rod flexural rigidity. This research effort did not attempt to account for and quantify each of these influences. Thus, the preliminary conclusion based on CIRFT SNF static bending testing is that the flexural rigidity was approximately twice what would be obtained if cladding properties alone were used to predict behavior.

From a flexural rigidity perspective, the fuel pellets indeed increase the SNF rod's system flexural rigidity; however, due to segmental pellet structure, numerous stress concentration sites within cladding at pellet-pellet interfaces in a SNF rod are created. In general the stress concentration event will cause the structure accelerated aging or reduced lifetime as compared to the very same system w/o stress concentration sites or discontinuous materials interfaces. The stress concentration effect due to an HBU rod segment structure was further validated from the dynamic testing, where the CIRFT test specimens are all failed at pellet-pellet interfaces. Therefore, the pellet and clad mechanical interaction at material discontinuity interface regions seems to reduce the benefit gained from the stiffness increase of a HBU fuel rod system. Moreover, the intensity of the stress concentration or the pellet-clad interaction is strongly dependent on the loading intensity. Thus, at low loading level, cladding alone is still the key that dictates the SNF rod flexural reliability. From CIRFT dynamic testing, it also confirms that as long as the clad fracture threshold was not reached, a SNF system can survive millions of vibration cycles.

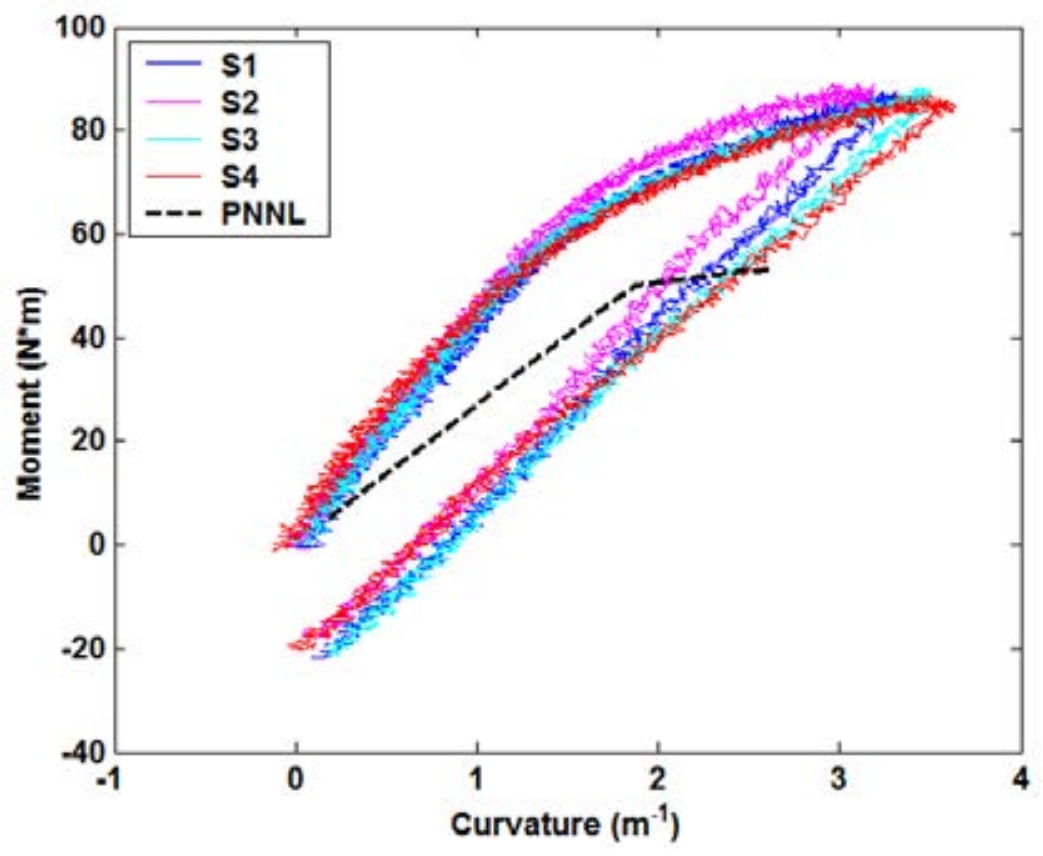

Figure 16. Comparison of CIRFT global data with PNNL moment-curvature curve converted from PNNL cladding stress-strain data. 
Table 6. Comparison of flexural rigidity results in CIRFT tests and PNNL data

\begin{tabular}{|l|l|l|l|}
\hline & El1 $\left(\mathbf{N} \cdot \mathbf{M}^{\mathbf{2}}\right)$ & El2 $\left(\mathbf{N} \cdot \mathbf{M}^{\mathbf{2}}\right)$ & El3 $\left(\mathbf{N} \cdot \mathbf{M}^{\mathbf{2}}\right)$ \\
\hline CIRFT testing & 54.751 & 39.944 & 33.090 \\
\hline PNNL data & 26.933 & & \\
\hline
\end{tabular}

\subsubsection{Effect of LVDT Probe Contact on Large Curvature Measurement}

The LVDT probe contact design with respect to monitoring specimen curvature deformation was found to affect curvature measurement results.

An experimental study was performed using two surrogate rods made of identical materials and configurations (stainless steel [SS] tubes and ten alumina pellets in each surrogate rod). The same flat disk probe contact was used to test each specimen. The flat disk probe forms a linecontact when the probe initially contacts the rod surface. However, upon loading, the contact will shift from the line-contact to point-contact at one side of the probe disk. As shown in Figure 17, under similar loading levels, the maximum curvature obtained from the LVDT probes located at a cladding compression site is significantly larger than that of probes located at cladding tension sites. The discrepancy due to the probe contact effect can be mitigated or reconciled by adjusting sensor spacing, $h$, of Eq. (1) as illustrated in Figure 18. It has been shown that under progressive adjustments the moment-curvature curves in both cases seem to converge. The actual sensor spacing adjustment can be calibrated with a chisel head probe that has point contact instead of line contract to the rod, as shown in Section 2.3.

Due to LVDT probes located at cladding compression sites during the static testing, the estimated curvature is expected to be overestimated as demonstrated in Figure 18 of $\mathrm{dh}=0 \mathrm{~mm}$ case. The resultant higher curvature trend seems to be consistent with the observations in Figure 17, where modified equivalence data corresponds to a higher peak tensile strain compared to that of the PNNL data.
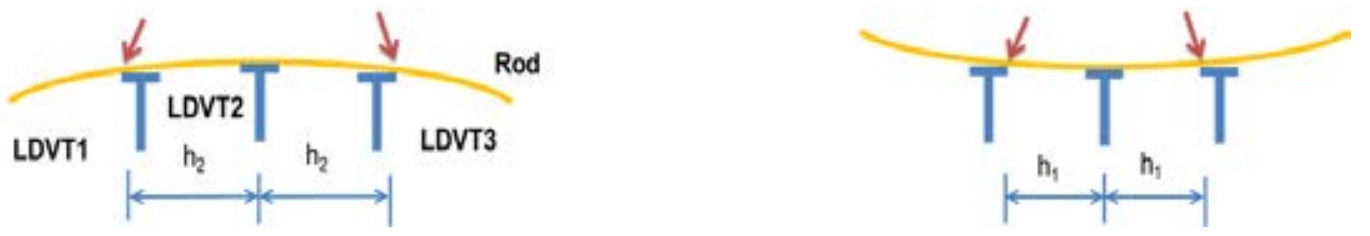

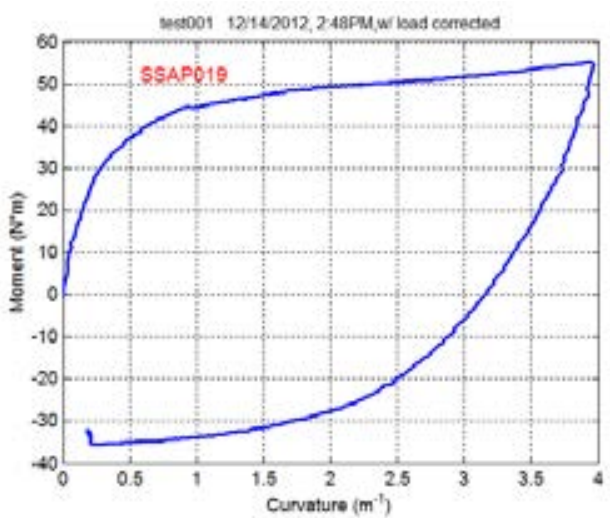

(a)

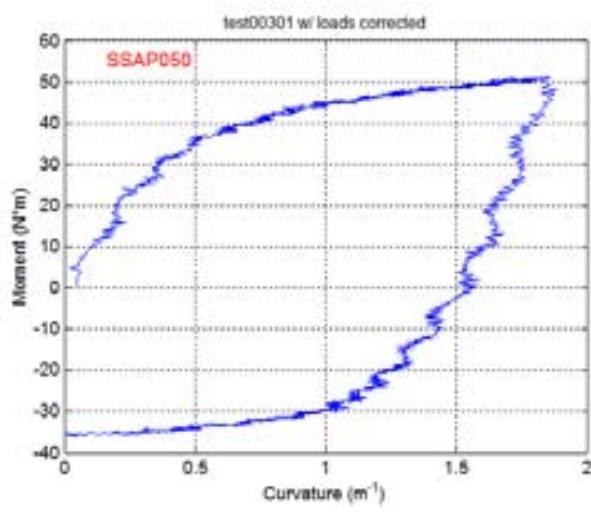

(b)

Figure 17. Moment-curvature curves based on stainless steel alumina pellet (SSAP) rod testing when rod is bending with respect to three LVDTs (a) concavely and (b) convexly. 

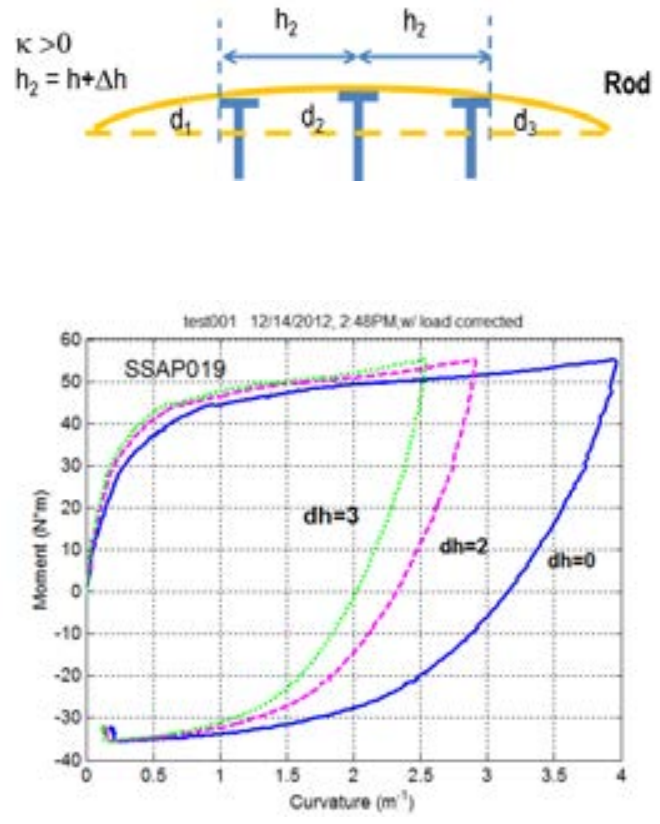

(a)
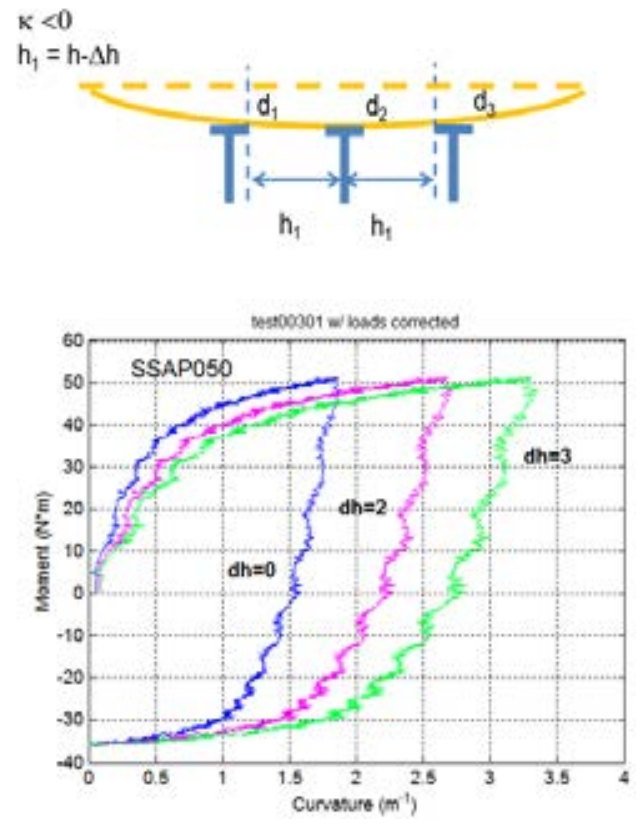

(b)

Figure 18. Moment-curvature curves as a function of sensor spacing adjustment when rod is bending with respect to three LVDTs (a) concavely and (b) convexly. The unit of dh is $\mathrm{mm}$. 



\section{DYNAMIC TESTING}

\subsection{RESULTS}

Sixteen dynamic tests were conducted on the HBU HBR fuel in the hot cell, including one for benchmark testing and fifteen according to dynamic testing plan. Load amplitudes varied from \pm 5.08 to $\pm 35.56 \mathrm{~N} \cdot \mathrm{m} ; 12$ tests were completed with specimen failure and 4 without failure. The fatigue life ranged from $5.5 \times 10^{3}$ to $2.3 \times 10^{6}$ cycles. The tests without failure were conducted under \pm 5.08 to $\pm 8.89 \mathrm{~N} \cdot \mathrm{m}$ with the accumulated cycles beyond $6.4 \times 10^{6}$. Details of the dynamic tests can be found in Appendix E; only typical results are described in this section.

The test on D1 (607C4B, $63.8 \mathrm{GWd} / \mathrm{MTU}$ burnup, 70-100 $\mu \mathrm{m}$ oxide layer) was conducted under $\pm 15.24 \mathrm{~N} \cdot \mathrm{m}, 5 \mathrm{~Hz}$. A fatigue life of $1.1 \times 10^{5}$ cycles was obtained, and less than 1 gram of fuel fragments was collected at the failure site.

For each cyclic fatigue test, periodic quasi-static measurements of rod deformation were conducted using two relative displacement levels: 0.8 and $1.2 \mathrm{~mm}$, at the target intervals described in Section 2.5.2. Time series of moment and curvature and moment-curvature loops obtained at the $1^{\text {st }}$ cycle and after approximately 111,000 cycles are shown in Figure 19 . The loops became slender with accumulated cycles, and the amplitude of the loops decreased. The relations of moment-range versus curvature-range and flexural rigidity are illustrated in Figure 20. Most of the rigidity degradation occurred in the first 1,000 cycles. Variations of these quantities as a function of number of cycles are provided in Figure 21. Although the curvature range stayed quite consistent under displacement control, a decrease in moment range was observed. The rigidity of the measurements at two displacements, converged before the failure while exhibiting a slightly declining trend.

The curvature, moment, and flexural rigidity based on online monitoring data are presented in Figure 22. The online monitoring showed a flexural rigidity of about $50 \mathrm{~N} \cdot \mathrm{m}^{2}$, a little lower than that observed in measurements. This occurred because different loading conditions were used in measurement and cycling. A curvature range of less than $0.3 \mathrm{~m}^{-1}$ was used in the quasi-static measurement, which is lower than that used in the cyclic test to ensure measurement data didn't affect the dynamic cycle data. In general, the flexural rigidity tends to increase with decreasing curvature. This is probably due to a better interface bond at relatively low load resulting in less stiffness reduction. Overall, a stable rod response was exhibited before the final failure. The curvature time history shown in Figure 24 (d), it clearly indicated a non-symmetric deformation under reverse loading, where the cladding tension site has much higher deformation (about 1.6 times) compared to that at cladding compression site. This phenomenon could be the consequence of debonding at the pellet-pellet interface and the stress concentration occurring at the pellet-pellet interface region at the tension side of the cladding, in addition to sensor probe sensitivity. In general flexural hysteresis history remains quite uniform throughout the reversal bending test, as shown in Fig. 24 (f). Under relatively low loading amplitude the clad or fuel pellet would be mainly under linear elastic behavior, the root cause of hysteresis energy dissipation under cyclic loading could be the system non-linear response associated with segment pellets induced stress concentration at interface regions as well as uncertainly resulting from sensor probe sensitivity.

Moment and curvature time history and moment-curvature loops based on online monitoring at 26 and $1.10 \times 10^{5}$ cycles are shown in Figure 23. The curvature data appear to have a much higher noise level compared to that of SSAP out-of-cell test data, as shown in Figure 10. This is 
primarily due to there being two different calibration ranges for the LVDT set-ups used in the out-of-cell testing. The large range set-up is designated for static testing due to the large deformation required in that type of test, and the small range set-up which has higher sensitivity is designed for dynamic testing due to its lower test specimen deformation level. In a hot-cell environment, the current CIRFT device is only equipped with the large range calibration LVDT set-up, which is used to cover both static and dynamic testing capabilities. Thus, the sensitivity of the small range set-up for the low load dynamic testing was sacrificed, resulting in the higher noise level in the curvature data of Figure 23. It is also noted that the symmetry curvature responses were observed at the beginning of cycles, which may indicate good interface bonds at the beginning of test cycle, while at higher cycling, the nonsymmetry characteristic of curvatures under clad tension and compression cycles was observed in Fig. 25.

The failure in test D1 was observed in the gage section near motor 2 (left side of the U-frame setup). The failure occurred at the pellet-to-pellet interface as illustrated in Figure 24 . The end faces of the two neighboring pellets were essentially clean. Both of the stressed cylinder surfaces of the rod were found to have been covered with equally spaced circumferential cracks throughout the gage section. Spalling only occurred on the local area near the fracture. The degree of damage shown on both of the stressed cylinder surfaces of specimen D1 is different from the damage experienced in test specimens undergoing only unidirectional bending. The large deformation arising from the unidirectional bending can produce a greater extent of damage or spalling on one side of the test specimen with much less spalling on the other side of the specimen (for example, see S3 [609C5] in Table 3). 

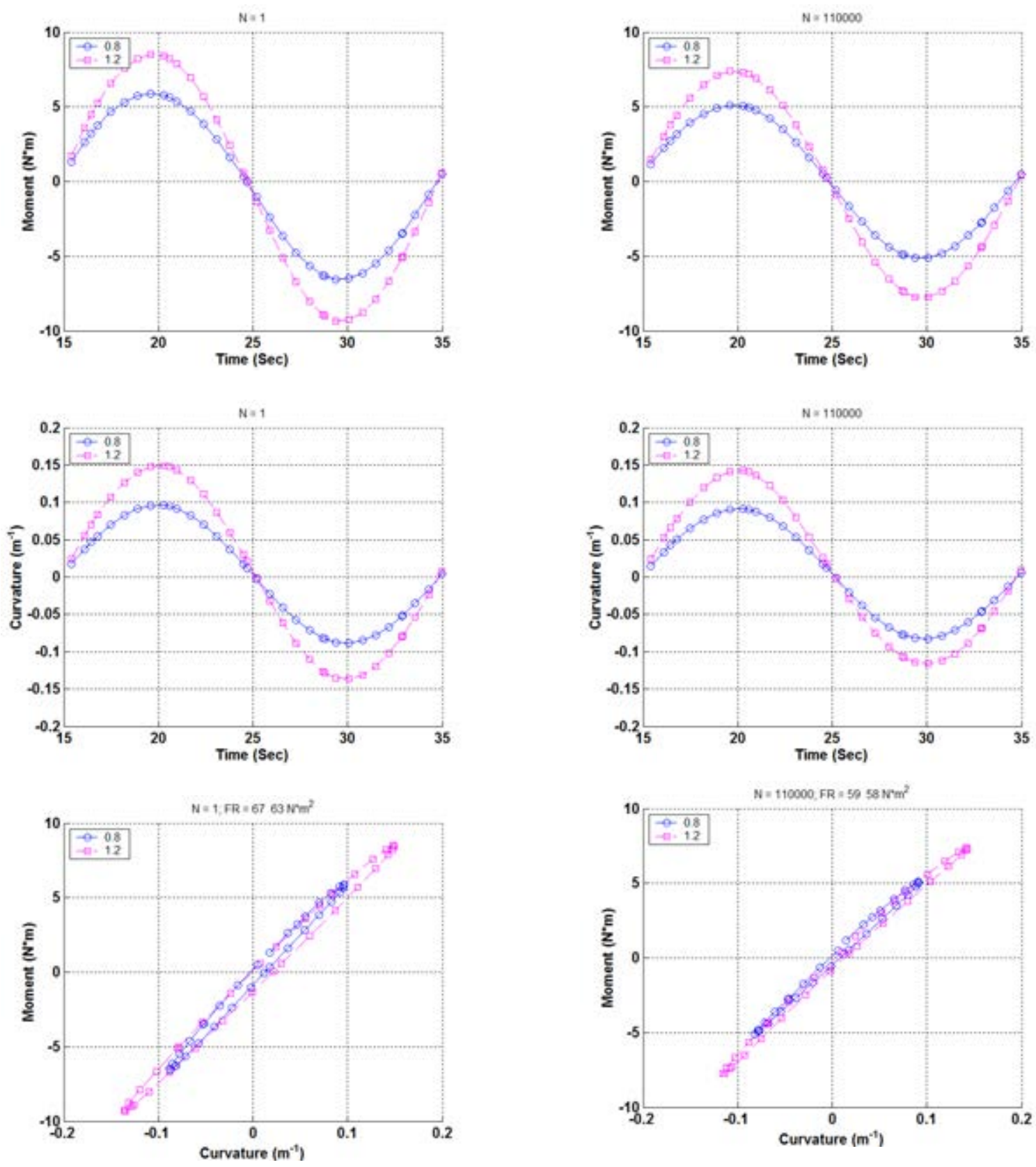

(a)

(b)

Figure 19. Moment and curvature as a function of time and moment-curvature loops based on measurements when (a) $N=1$ and (b) $N=111,000$ cycles for D1 (607C4B). Measurements were made with 0.8 and $1.2 \mathrm{~mm}$ relative displacements; $\mathrm{N}_{\mathrm{f}}=1.1 \times 10^{5}$ cycles under $\pm 15.24 \mathrm{~N} \cdot \mathrm{m}, 5 \mathrm{~Hz}$. Fuel particles collected $<1.0 \mathrm{~g}$. 


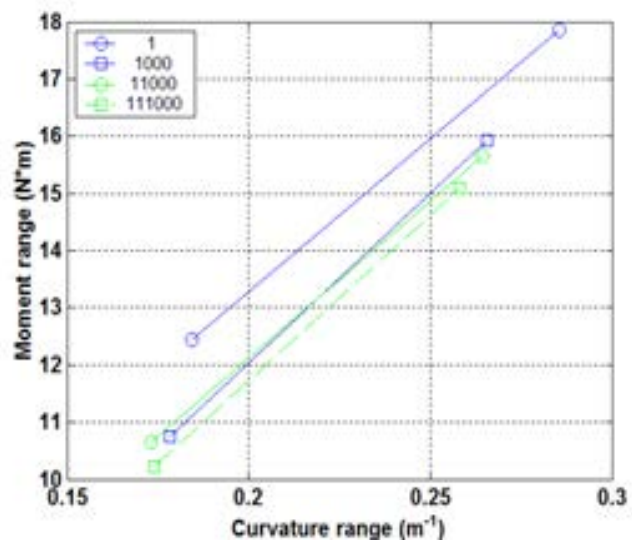

(a)

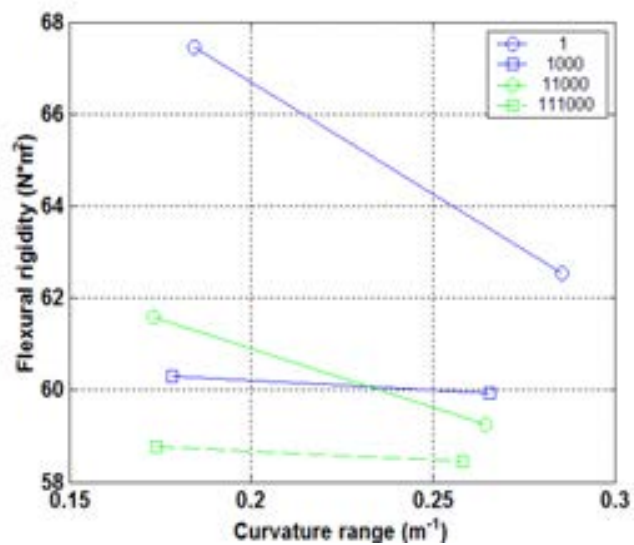

(b)

Figure 20. (a) Moment-curvature relation and (b) moment-flexural rigidity relation at various numbers of cycles for $\mathrm{D} 1(607 \mathrm{C} 4 \mathrm{~B}) ; \mathrm{N}_{\mathrm{f}}=1.1 \times 10^{5}$ cycles under $\pm 15.24 \mathrm{~N} \cdot \mathrm{m}, 5 \mathrm{~Hz}$. Fuel particles collected $<1.0 \mathrm{~g}$.

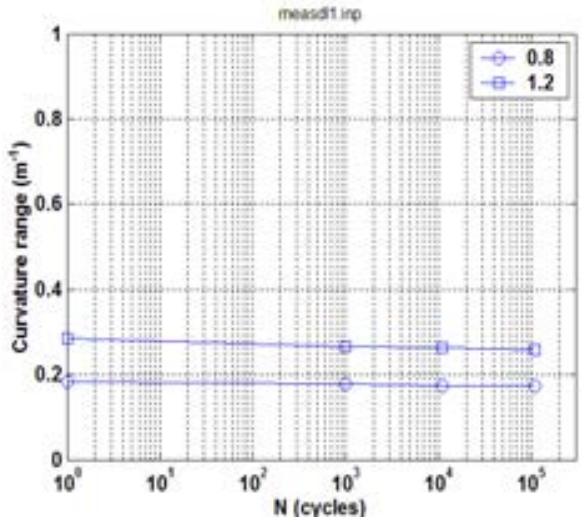

(a)

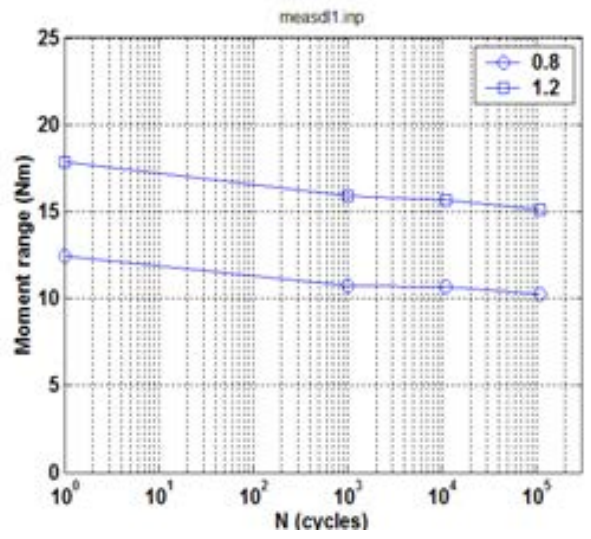

(b)

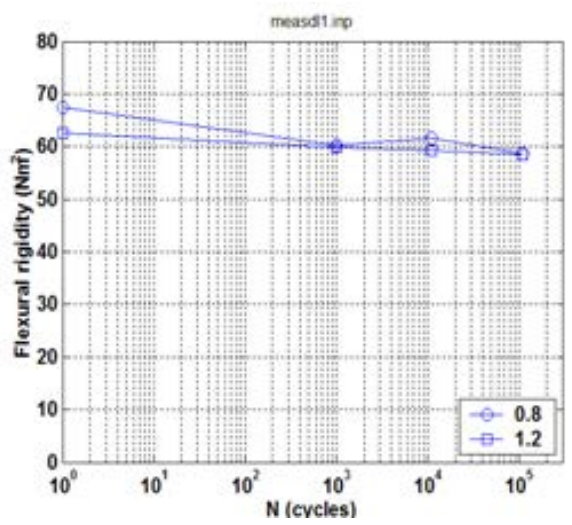

(c)

Figure 21. Variations of (a) curvature range, (b) moment range, (c) flexural rigidity as a function of number of cycles for D1 (607C4B); $\mathrm{N}_{\mathrm{f}}=1.1 \times 10^{5}$ cycles under $\pm 15.24 \mathrm{~N} \cdot \mathrm{m}, 5 \mathrm{~Hz}$. Fuel particles collected $<1.0 \mathrm{~g}$. 


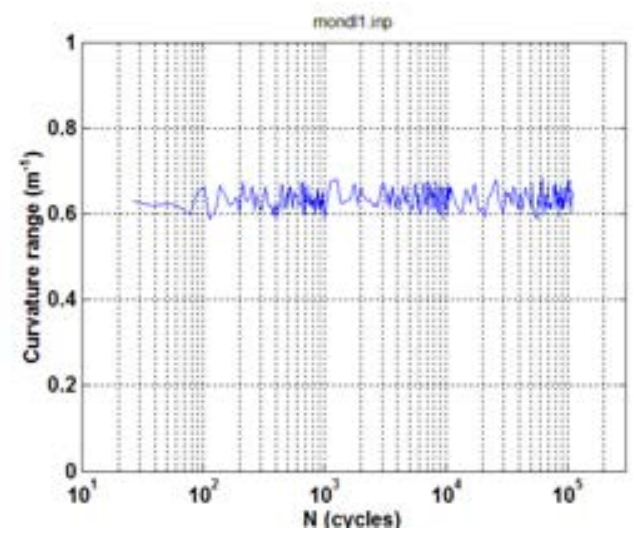

(a)

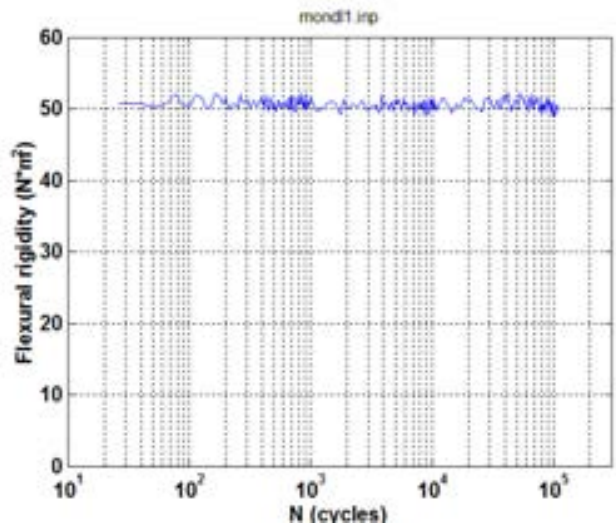

(c)

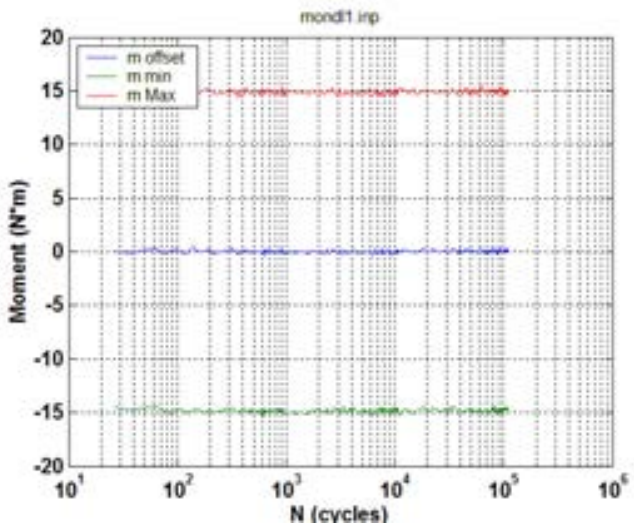

(e)

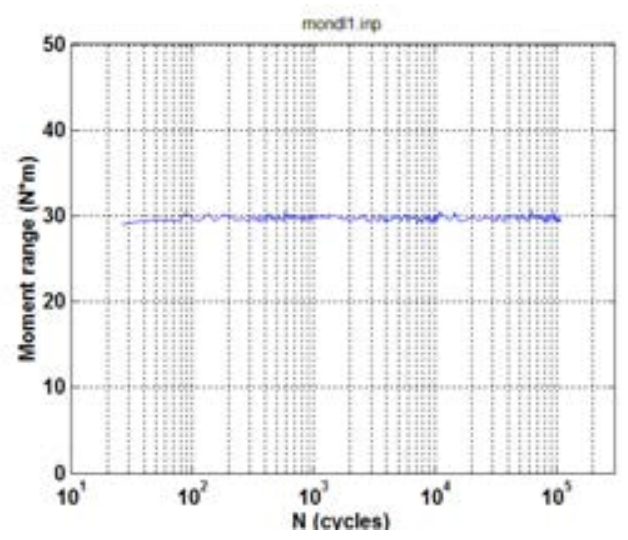

(b)

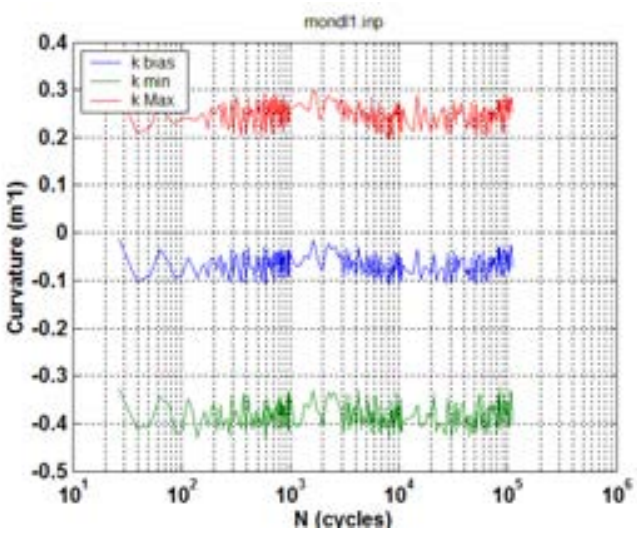

(d)

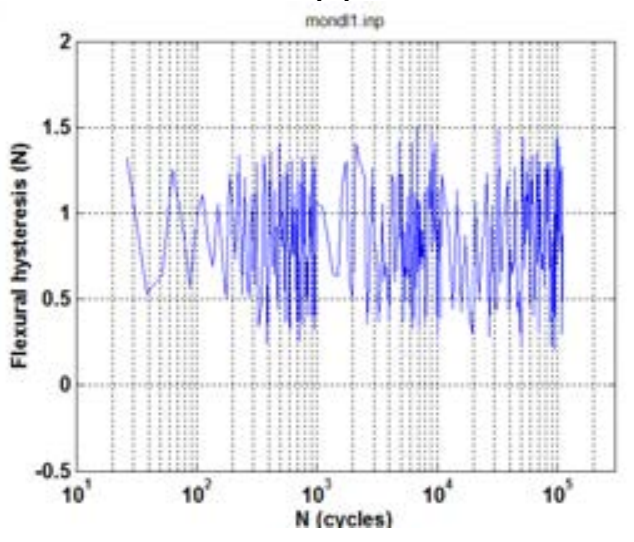

(f)

Figure 22. Variations of (a) curvature range, (b) applied moment range, (c) flexural rigidity, (d) maximum and minimum values of curvature, (e) maximum and minimum values of moment, and ( $f$ ) flexural hysteresis as a function of number of cycles for D1 $(607 \mathrm{C} 4 \mathrm{~B}) ; \mathrm{N}_{\mathrm{f}}=1.1 \times 10^{5}$ cycles under $\pm 15.24 \mathrm{~N} \cdot \mathrm{m}, 5 \mathrm{~Hz}$. Fuel particles collected $<1.0 \mathrm{~g}$. 

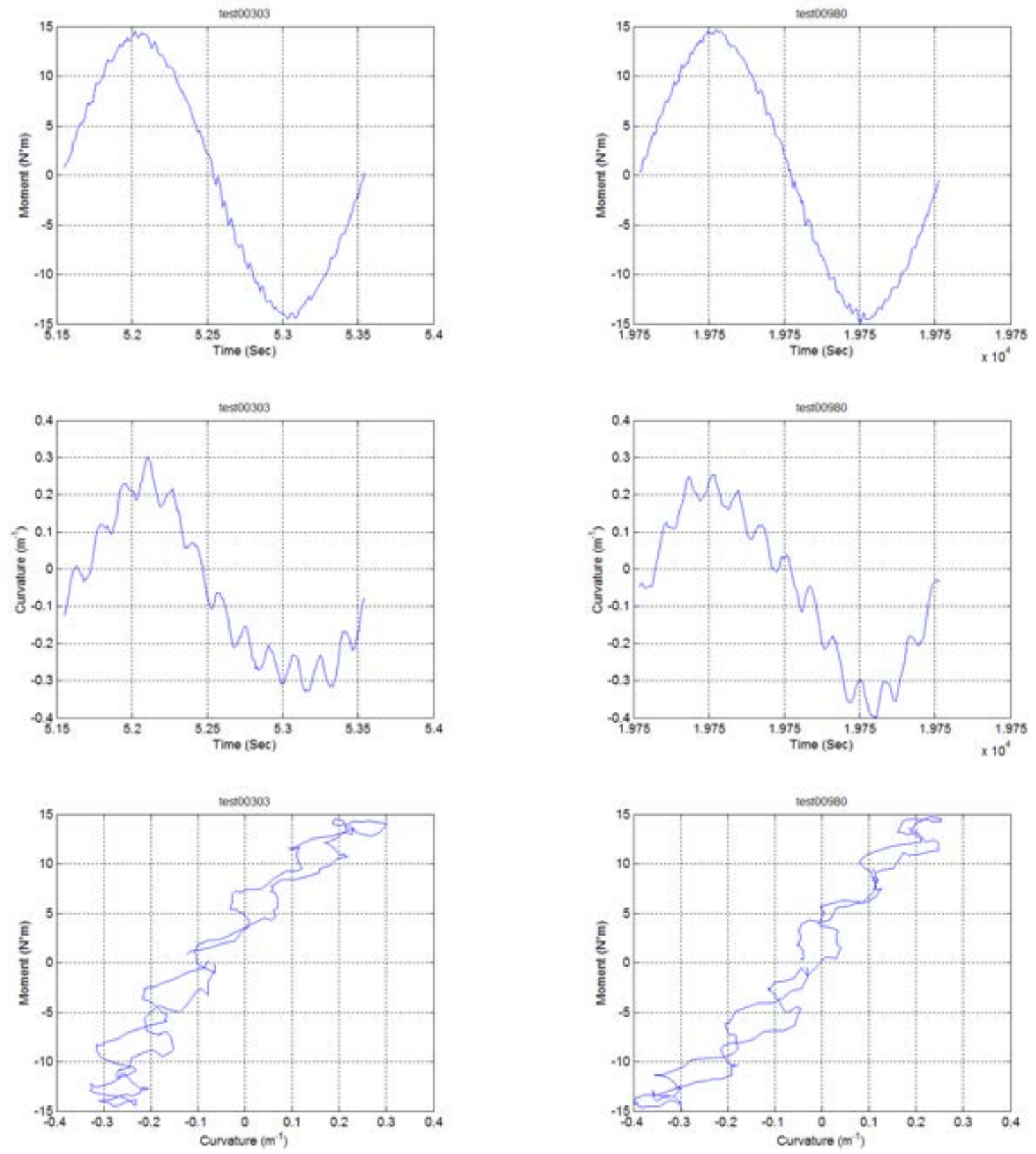

(a)

(b)

Figure 23. Moment and curvature as a function of time and moment-curvature loops at (a) 26 and (b) $1.10 \times 10^{5}$ cycles; results are based on online monitoring. 


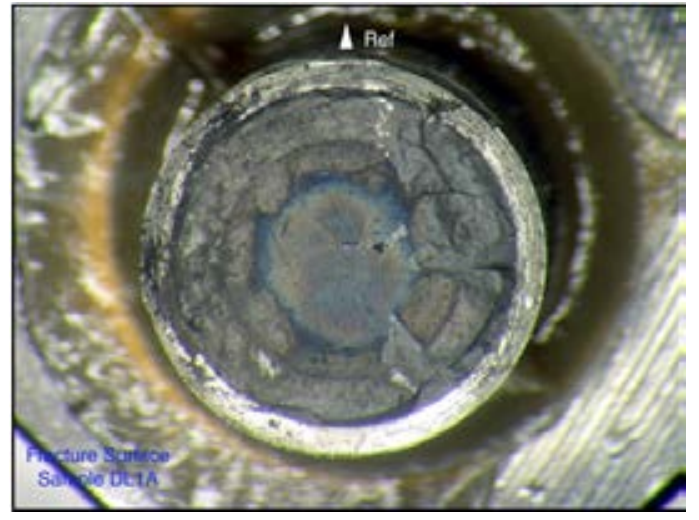

(a)

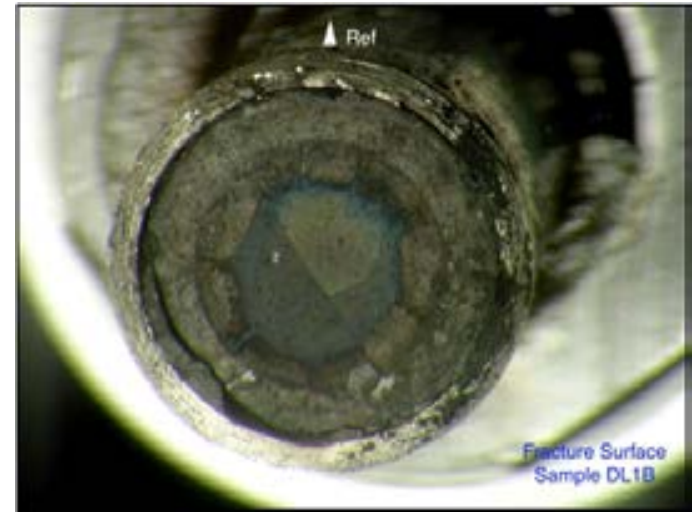

(b)
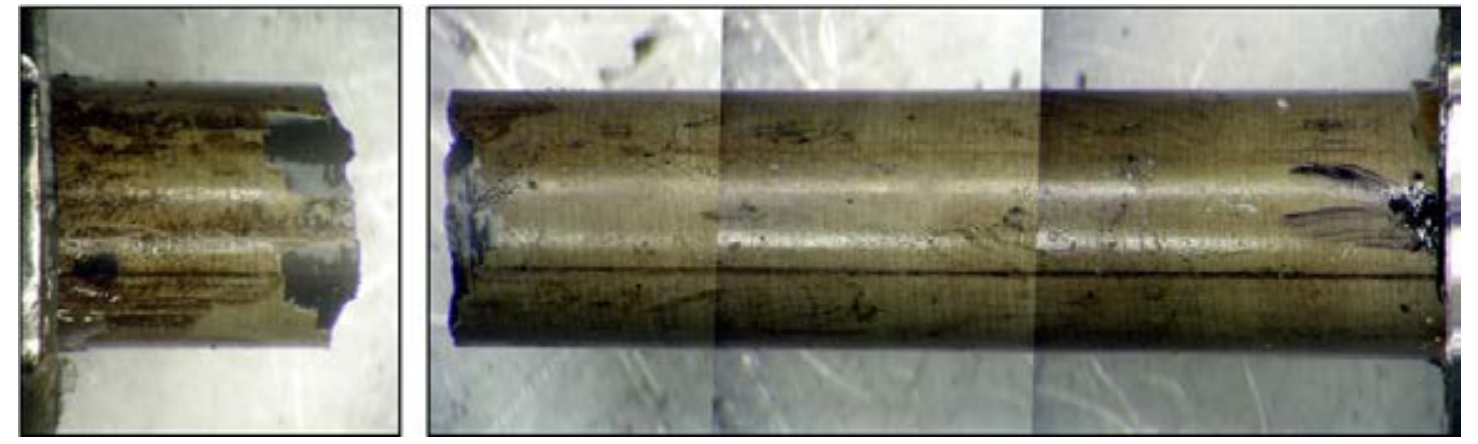

(c)
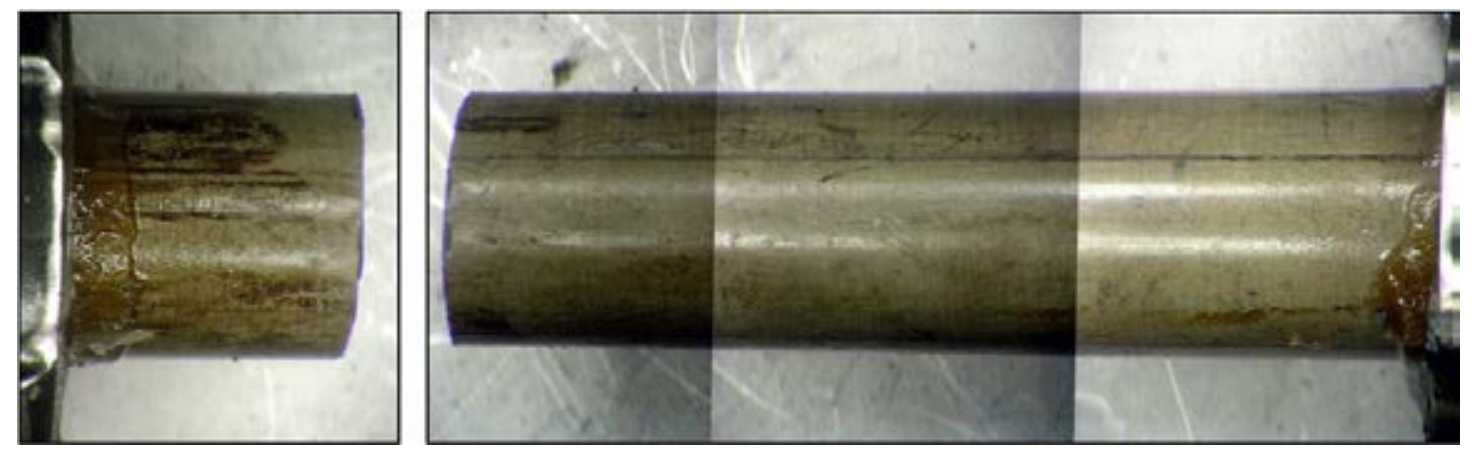

(d)

Figure 24. (a) and (b) Mating fracture surfaces, (c) frontal, and (d) back sides for D1 $(607 \mathrm{C} 4 \mathrm{~B}), \mathrm{N}_{\mathrm{f}}=1.1 \times 10^{5}$ cycles under $\pm 15.24 \mathrm{~N} \cdot \mathrm{m}, 5 \mathrm{~Hz}$. Fuel particles collected $<1.0 \mathrm{~g}$.

The details of the other dynamic tests can be seen in Appendix $E$. The dynamic testing results for HBR rods are summarized in Table 7, along with one rod from benchmarking (D0). The result for specimen DO is considered to be effective in characterizing the cyclic fatigue of rods, as the test followed the dynamic testing procedure. Table 7 shows the failure modes of rods from the dynamic tests. 


\begin{tabular}{|c|c|c|c|c|c|c|c|c|c|c|c|c|c|c|c|}
\hline 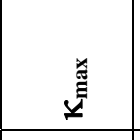 & $\boldsymbol{r}$ & $\begin{array}{l}8 \\
0 \\
1 \\
0 \\
0\end{array}$ & 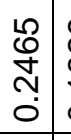 & 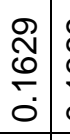 & 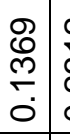 & \begin{tabular}{c|c}
\multirow{2}{*}{} \\
\multirow{N}{0}{} \\
0
\end{tabular} & 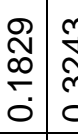 & 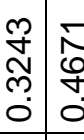 & 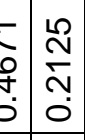 & 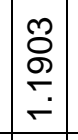 & 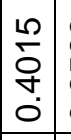 & 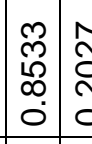 & \begin{tabular}{c|c} 
& $m$ \\
& 0 \\
& 0 \\
0 & 0 \\
0 & 0 \\
\end{tabular} & 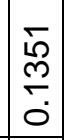 & 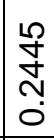 \\
\hline 章 & I & $\begin{array}{c}\text { 芯 } \\
\text { గొ } \\
0 \\
1\end{array}$ & 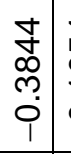 & 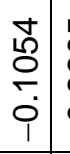 & 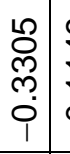 & 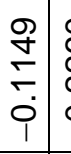 & 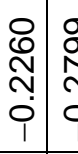 & 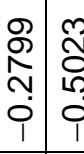 & 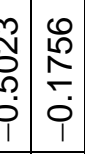 & $\begin{array}{l}0 \\
\stackrel{\sim}{0} \\
0 \\
0 \\
1\end{array}$ & \begin{tabular}{|l|}
0 \\
$\tilde{m}$ \\
0 \\
0 \\
0 \\
1
\end{tabular} & 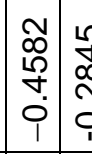 & 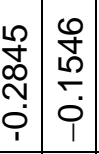 & 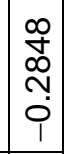 & $\begin{array}{l}\hat{\Omega} \\
\text { o } \\
0 \\
0\end{array}$ \\
\hline$\underline{\Xi}$ & $\boldsymbol{r}$ & \begin{tabular}{l}
1 \\
\multirow{1}{f}{} \\
0 \\
0 \\
0
\end{tabular} & $\begin{array}{l}8 \\
0 \\
0 \\
0 \\
0 \\
1\end{array}$ & \begin{tabular}{l|l}
$\infty$ & \\
$\mathbb{D}$ & \\
0 & \\
0 & \\
\end{tabular} & \begin{tabular}{l|l}
$\infty$ & \\
0 & \\
$\circ$ & \\
$\circ$ & \\
0 & \\
& \\
\end{tabular} & 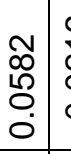 & 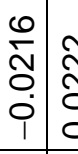 & 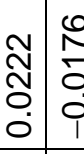 & 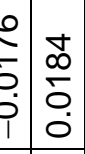 & 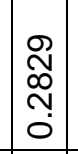 & $\begin{array}{l}8 \\
8 \\
\circ \\
0 \\
0\end{array}$ & 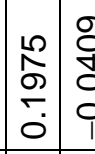 & 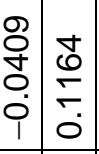 & 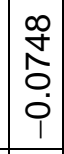 & \begin{tabular}{l}
\multirow{N}{N}{} \\
0 \\
0
\end{tabular} \\
\hline$\frac{\mathscr{*}}{\underline{E}}$ & ' & $\begin{array}{l}8 \\
0 \\
0 \\
0 \\
0\end{array}$ & \begin{tabular}{c}
\multirow{D}{*}{} \\
$D$ \\
$\tilde{m}$ \\
$\dot{0}$
\end{tabular} & 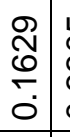 & 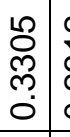 & 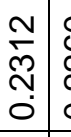 & 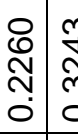 & 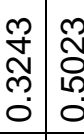 & 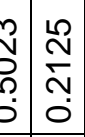 & 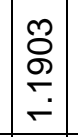 & 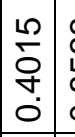 & \begin{tabular}{lll}
$\prod_{n}$ & \multicolumn{1}{c}{} \\
$\infty$ & $\alpha$ \\
0 & $\vdots$ \\
0 & 0
\end{tabular} & 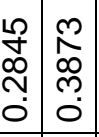 & 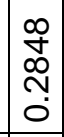 & 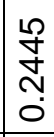 \\
\hline$\stackrel{N}{\ddot{y}}$ & II & 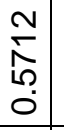 & $\begin{array}{c}m \\
N \\
-1 \\
0 \\
0\end{array}$ & $\begin{array}{c}m \\
\stackrel{m}{-} \\
\stackrel{0}{0}\end{array}$ & 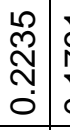 & 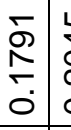 & 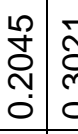 & 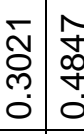 & 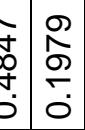 & \begin{tabular}{l}
\multirow{N}{*}{} \\
8 \\
0 \\
0
\end{tabular} & $\begin{array}{c}0 \\
\tilde{N} \\
D \\
\tilde{D} \\
0\end{array}$ & 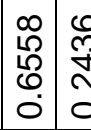 & 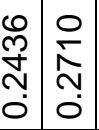 & 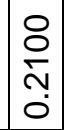 & ㄱ. \\
\hline$\frac{\mathfrak{N}}{\omega}$ & $\circ$ & \begin{tabular}{l}
$m$ \\
\multirow{2}{*}{} \\
0 \\
0 \\
0
\end{tabular} & 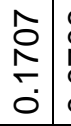 & $\begin{array}{l}0 \\
⿱ 乛 \\
0 \\
0\end{array}$ & 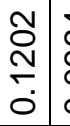 & 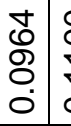 & 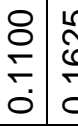 & 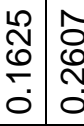 & 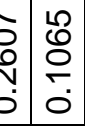 & \begin{tabular}{c}
$\widetilde{1}$ \\
$\infty$ \\
$\infty$ \\
\multirow{\sigma}{*}{} \\
0
\end{tabular} & 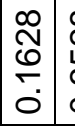 & 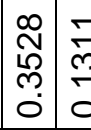 & 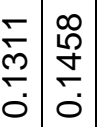 & $\begin{array}{l}\stackrel{0}{m} \\
\stackrel{m}{-} \\
\stackrel{0}{0}\end{array}$ & 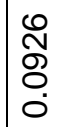 \\
\hline$\stackrel{\pi}{\leftarrow} *$ & & -1 & -1 & 0 & $\rightarrow$ & 0. & $r$ & -1 & \begin{tabular}{r|l}
-1 & 0 \\
\end{tabular} & -1 & -1 & $-1-$ & $-1-1$ & -1 & 0 \\
\hline 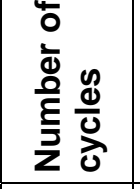 & & 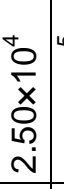 & 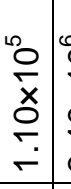 & \begin{tabular}{l|l}
0 & 0 \\
& \\
$\times$ & \\
0 & \\
+ & \\
0 &
\end{tabular} & 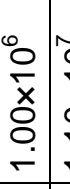 & 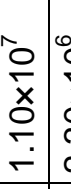 & 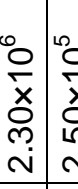 & 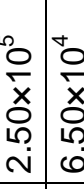 & 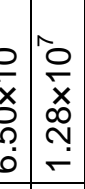 & $\mid \begin{array}{c}0 \\
0 \\
-1 \\
x \\
0 \\
\\
\end{array}$ & 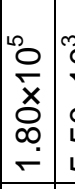 & 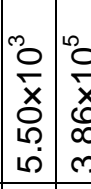 & 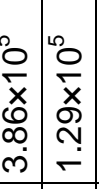 & 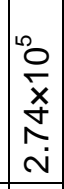 & $\begin{array}{l}0 \\
0 \\
\vec{y} \\
x \\
-7 \\
-1 \\
10\end{array}$ \\
\hline 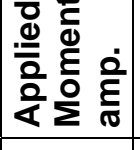 & $\frac{\xi}{\dot{z}}$ & $\stackrel{ナ}{\stackrel{D}{N}}$ & 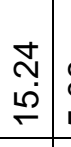 & 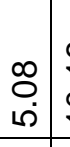 & 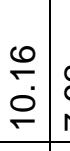 & 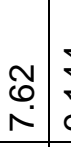 & 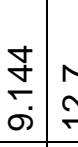 & 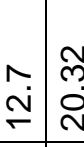 & \begin{tabular}{l|l}
$v$ & $\infty$ \\
& $\infty$ \\
& $\infty$ \\
\end{tabular} & 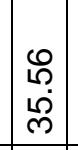 & \begin{tabular}{l|l}
$\sim$ & \\
\multirow{\sim}{\sim}{} &
\end{tabular} & 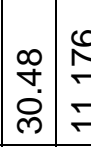 & 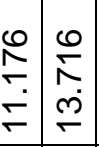 & $\begin{array}{l} \\
\infty \\
\infty \\
\infty\end{array}$ & $\underset{\widetilde{C}}{\widetilde{N}}$ \\
\hline 응 : & $\mathbf{z}$ & $\stackrel{\circ}{\stackrel{\circ}{\circ}}$ & \begin{tabular}{|l|}
0 \\
$\stackrel{1}{⿴ 囗 十}$ \\
\end{tabular} & 오 & ○ & $\stackrel{\sim}{\sim}$ & ৪ & $\underset{\sim}{\sim} \underset{\sim}{\circ}$ & 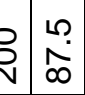 & 只 & 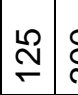 & ৪্ল & 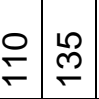 & 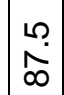 & م \\
\hline $\begin{array}{l}\text { 을 } \\
\text { \& }\end{array}$ & & 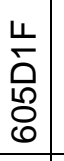 & 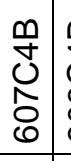 & 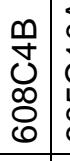 & \begin{tabular}{l|l} 
& \\
0 & \\
0 & \\
0 & \\
0 \\
0
\end{tabular} & 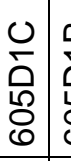 & 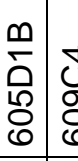 & 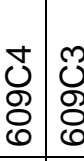 & 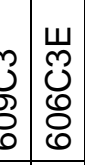 & $\begin{array}{l}\widehat{U} \\
8 \\
0 \\
0\end{array}$ & 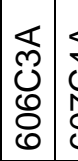 & 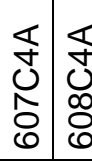 & 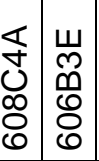 & $\begin{array}{l}0 \\
\tilde{p} \\
0 \\
0 \\
0\end{array}$ & $\begin{array}{l}0 \\
\tilde{n} \\
0 \\
0 \\
8\end{array}$ \\
\hline $\begin{array}{l}\Phi^{ \pm} \Phi \\
\text { ஸे }\end{array}$ & & 음 & (1) & $\tilde{\Delta}$ & $\stackrel{m}{2}$ & ¿ & $\begin{array}{l}L \\
\Delta\end{array}$ & \begin{tabular}{l|c}
0 \\
0
\end{tabular} & \begin{tabular}{|l|l} 
\\
\end{tabular} & 口̊ & 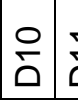 & $\stackrel{ન}{ન}$ & 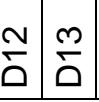 & $\mid \begin{array}{r}+ \\
\Delta\end{array}$ & $\stackrel{2}{\Delta}$ \\
\hline
\end{tabular}




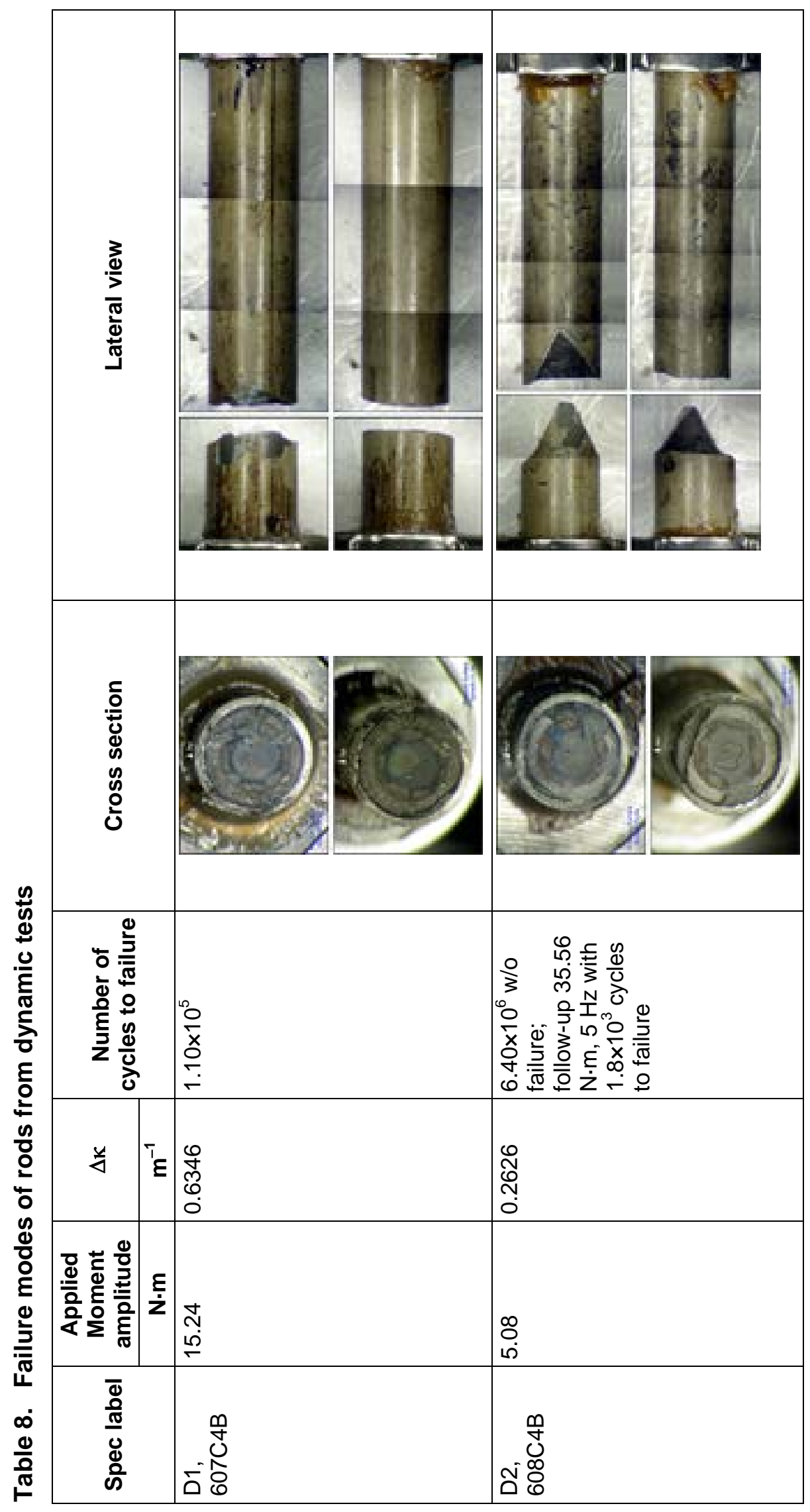




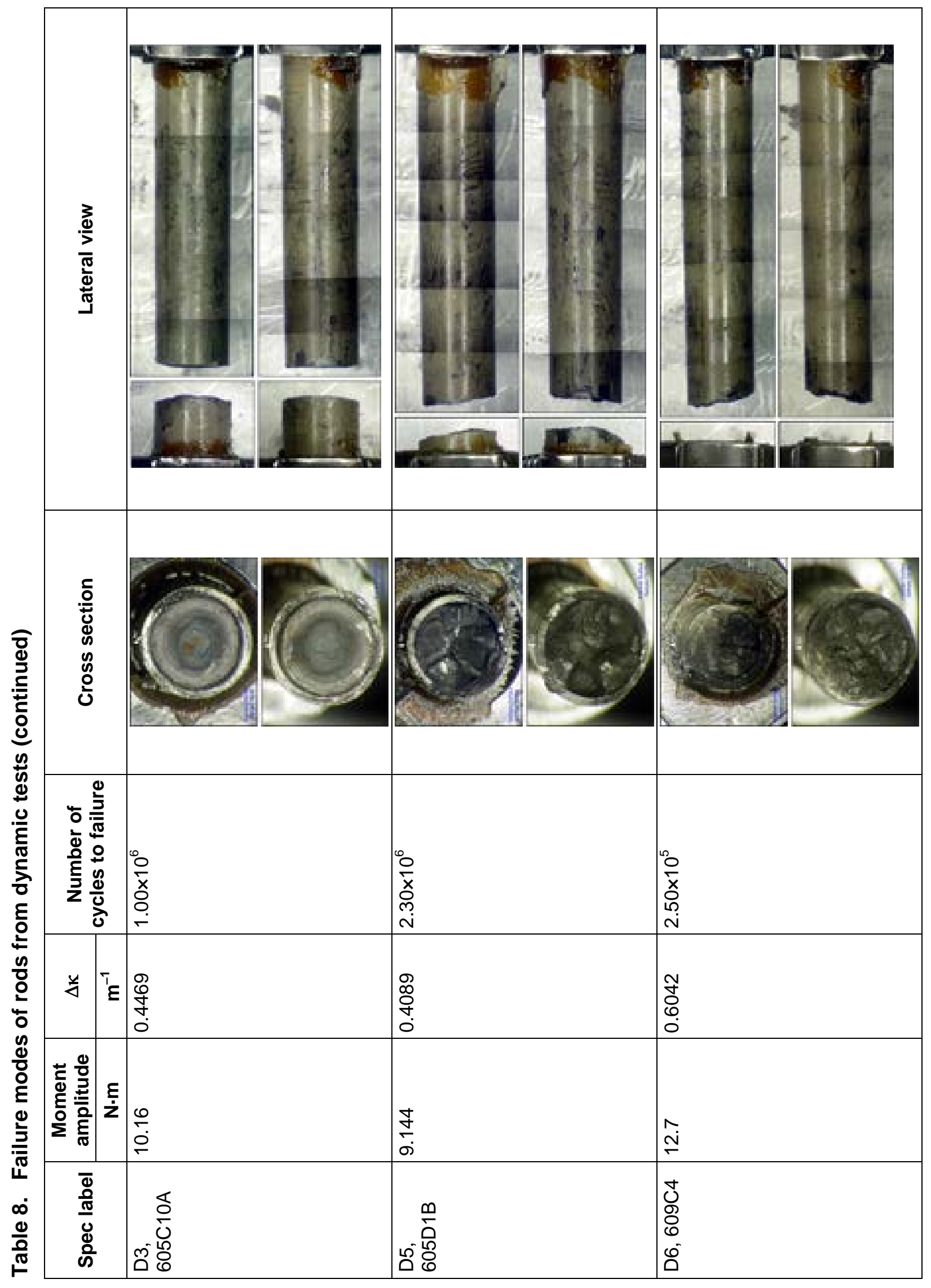




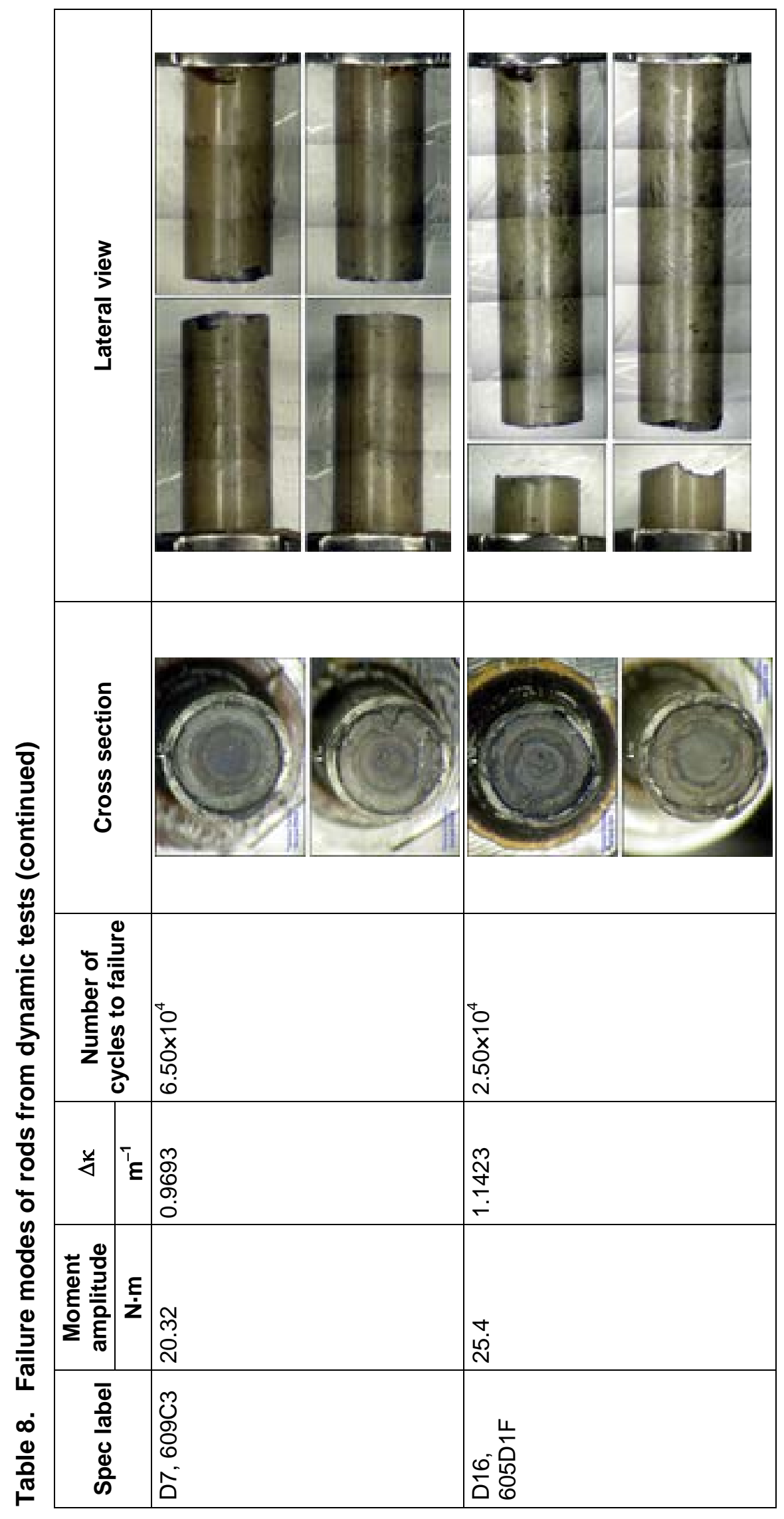


The equivalent strain amplitudes ( $\varepsilon_{\mathrm{a}}$, half of strain range $\Delta \varepsilon / 2$, and equivalent strain $\varepsilon$ is based on Eq. [4]) as a function of the number of failure cycles are shown in Figure 25.

- For those specimens that failed during testing, the fatigue life decreases with increasing strain amplitude, resulting in a defined $\varepsilon-\mathrm{N}$ curve. The data points can be effectively fit by using the power function $y=3.5693 \cdot x^{-0.252}$ with a correlation coefficient as high as 0.8722 , where $x$ is the number of cycles to failure and $y$ is the strain amplitude (\%).

- An endurance limit is likely located between 0.106 and $0.110 \%$ strains if it is defined at $10^{7}$ cycles. In other words, below $0.1 \%$ strain amplitude there appears to be no number of cycles that will cause failure.

- A large variation of hydrogen content existed in the cladding of the test specimens in the dynamic tests. However, the contribution of hydrogen content to the number of cycles to failure appears to be secondary to the effect of imposed loading amplitudes.

The curve fitting to the strain amplitude versus number of cycles was extended to include the data points without failure by using two power functions, as presented in Figure 26 . For $\mathrm{N} \leq 106$, the power function was that based on failure data points, $y=3.5693 \cdot x^{-0.252}$; for $N>10^{6}, y=$ $0.3234 \cdot x^{-0.076}$. It can be seen that the correlation efficient was quite lower for the second power function. This is because the amplitude is close to the endurance limit, and the number of failure cycles is likely independent of the applied load.

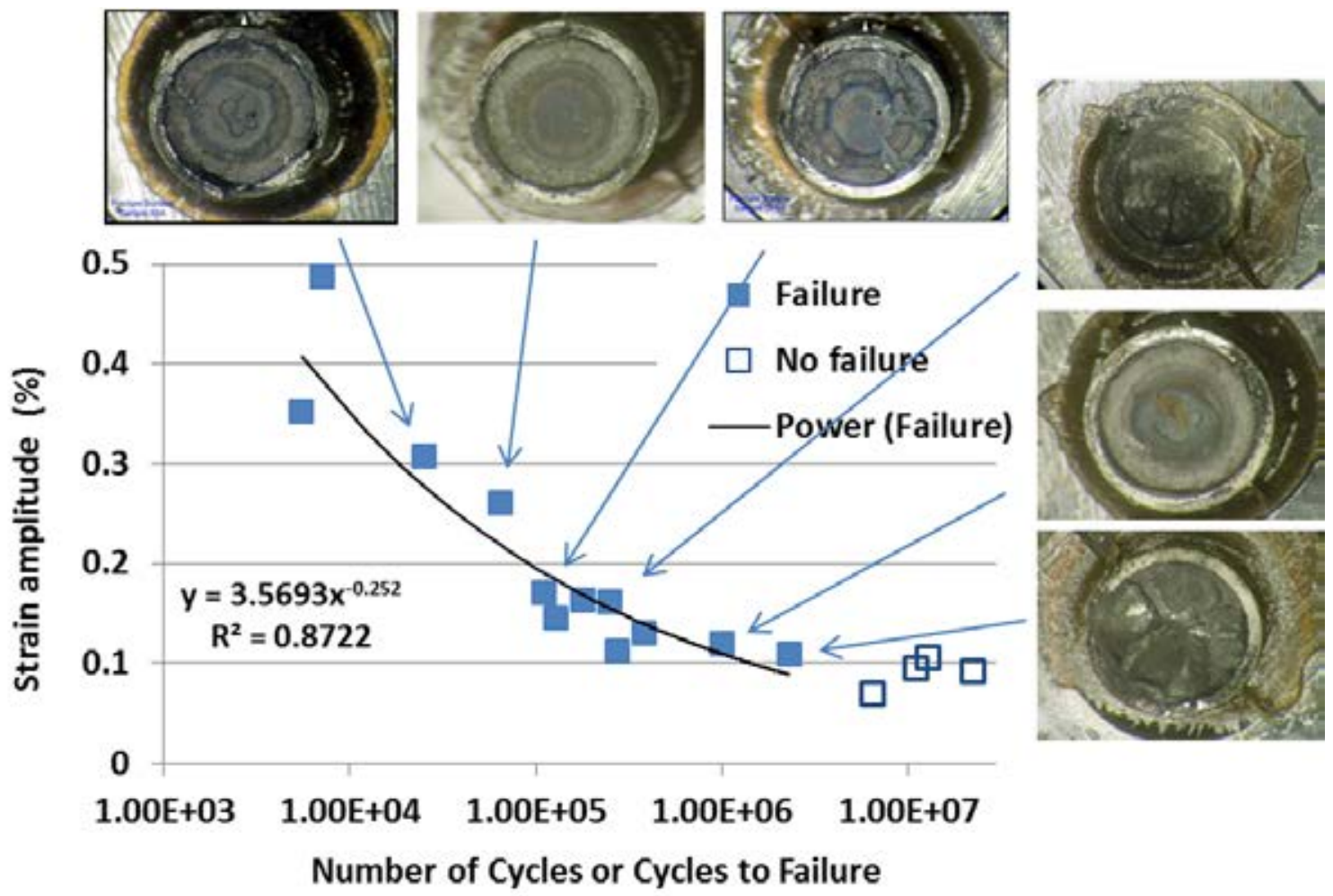

Figure 25. Equivalent strain amplitudes $(\Delta \varepsilon / 2)$ as a function of number of cycles. Solid markers represent tests with specimens failure; open markers indicate the tests stopped without failure. 


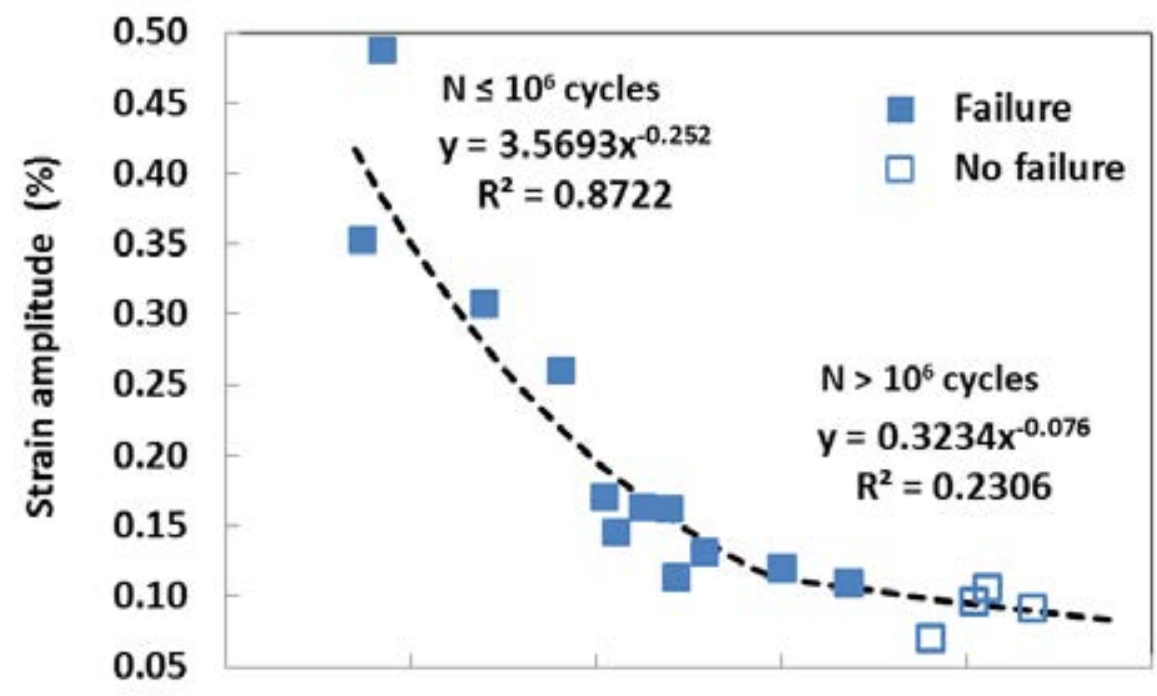

$\begin{array}{llllll}1.00 \mathrm{E}+03 & 1.00 \mathrm{E}+04 & 1.00 \mathrm{E}+05 & 1.00 \mathrm{E}+06 & 1.00 \mathrm{E}+07 & 1.00 \mathrm{E}+08\end{array}$

Number of Cycles or Cycles to Failure

Figure 26. Equivalent strain amplitudes $(\Delta \varepsilon / 2)$ as a function of number of cycles with curve fitting extended to include the data points without failure. Solid markers represent tests with specimens failure; open markers indicate the tests stopped without failure.

\subsection{DISCUSSION}

\subsection{1 к-N Curve}

The equivalent strain amplitude has been used to describe the loading condition imposed on the rods. The use of equivalent strain provides an expedient means to estimate the global response of the tested rod. However, the more elaborated localized deformation mechanism involved with pellet-pellet interfaces cannot be readily represented by this simplified approach. In order to provide a more accurate representation of a fatigue lifetime estimate (in addition to the curvature range), a new parameter of maximum curvature was defined. Due to dishing on the pellet end and the existence of an oxide contact interface, it is expected that HBU fuel has a fairly weak interface bond at the pellet-pellet interface. This phenomenon was also observed in CIRFT test results, where the flexural rigidity dropped off quickly in the initial cycles of testing and where a non-symmetric curvature response was revealed in the reverse loading cycle. Asymmetrical deformation response is an important signature of the HBU SNF due to pelletpellet-cladding interaction. The curvature at the cladding tensile stress site has the highest deformation during fatigue cycles, so it was used to develop the maximum curvature plots shown in Figure 27 to serve as another important index for the HBU SNF fatigue life.

The observations based on curvature amplitude ( $\kappa_{a}$, half of curvature range $\left.\Delta \kappa / 2\right)$ are similar to those of strain amplitude since the conversion simply involved a multiplication factor as shown in Eq. (4).

- The fatigue life decreases with increasing curvature amplitude with a defined $\kappa-\mathrm{N}$ curve. The curve fitting generated a power function $y=6.6345 \cdot x^{-0.252}$ with a correlation coefficient 0.8722 , where $x$ is the number of cycles to failure and $y$ is the curvature amplitude $\left(\mathrm{m}^{-1}\right)$. 
- An endurance limit may be located between 0.198 and $0.204 \mathrm{~m}^{-1}$ if it is defined at $10^{7}$ cycles.

It is worth noting that the dynamic tests were conducted under load control, and the curvature response of the rods was not necessarily symmetric (Figure 22 [d]). The mean values of the monitored mean curvatures $\kappa_{m}$ were generally not at the zero level as seen in Table 7 .

An investigation of the maxima of absolute values of curvature extremes $(|\kappa| \max$, defined in Section 2.6) was pursued as an alternative quantity for describing the loading imposed on fuel. This is significant because the maxima actually reflect the maximum tensile loading levels to which the specimen is subjected during the dynamic testing. The results are shown in Figure 27 (a). The $\kappa-\mathrm{N}$ curve has been demonstrated in terms of maxima of cladding curvature $|\kappa| \max$ to be similar to that of the curvature amplitude $\kappa_{a}$; the exponents of the curve-fit power function are in fact the same. The scatter plots based on the two approaches are similar with some changes in the relative positions of points between 1.1 and $3.9 \times 10^{5}$ cycles. Overall, the maxima of absolute curvature are larger than the curvature amplitude, depending on the magnitude of $\kappa_{\mathrm{m}}$.

The following observations can be made with regard to curvature maxima:

- For those specimens that failed during the tests, the fatigue life decreases with increasing curvature maxima according to a well-defined $\kappa-\mathrm{N}$ curve. The data points can be fit by using the power function $y=8.1941 \cdot x^{-0.252}$ with a correlation coefficient as high as 0.8926 , where $x$ is the number of cycles to failure and $y$ is the curvature maxima $\left(\mathrm{m}^{-1}\right)$.

- A fatigue limit may be located between 0.226 and $0.245 \mathrm{~m}^{-1}$ if it is defined at $10^{7}$ cycles.

- Due to the unsymmetrical deformation nature of HBU SNF under dynamic cycling, the bending neutral axis of the deformed rod is expected to shift away from the cladding tensile stress site. A first order approximation of simplified strain conversion from the curvature was used to derive the strain maxima vs. the number of failure cycles as shown in Figure 27 (b).

The curve fitting to the fatigue lifetime as a function of curvature amplitudes was extended to include the data points without failure by using two power functions, and the result is presented in Figure 28(a). For $\mathrm{N} \leq 10^{6}$, the power function was that based on failure data points, $y=6.6345 \cdot x^{-0.252}$; for $N>10^{6}, y=0.6011 \cdot x^{-0.076}$. The curve-fitting to the maxima of absolute curvatures resulted in the following power functions shown in Fig. 30(b). For $\mathrm{N} \leq 8 \times 10^{5}$, $y=8.1941 \cdot x^{-0.252}$; for $N>8 \times 10^{5}, y=0.9762 \cdot x^{-0.093}$. The curve-fitting to the maxima of absolute strains resulted in the following power functions, shown in Fig. 30 (c). For $\mathrm{N} \leq 8 \times 10^{5}$, $y=4.4084 \cdot x^{-0.252}$; for $N>8 \times 10^{5}, y=0.5252 \cdot x^{-0.093}$. 


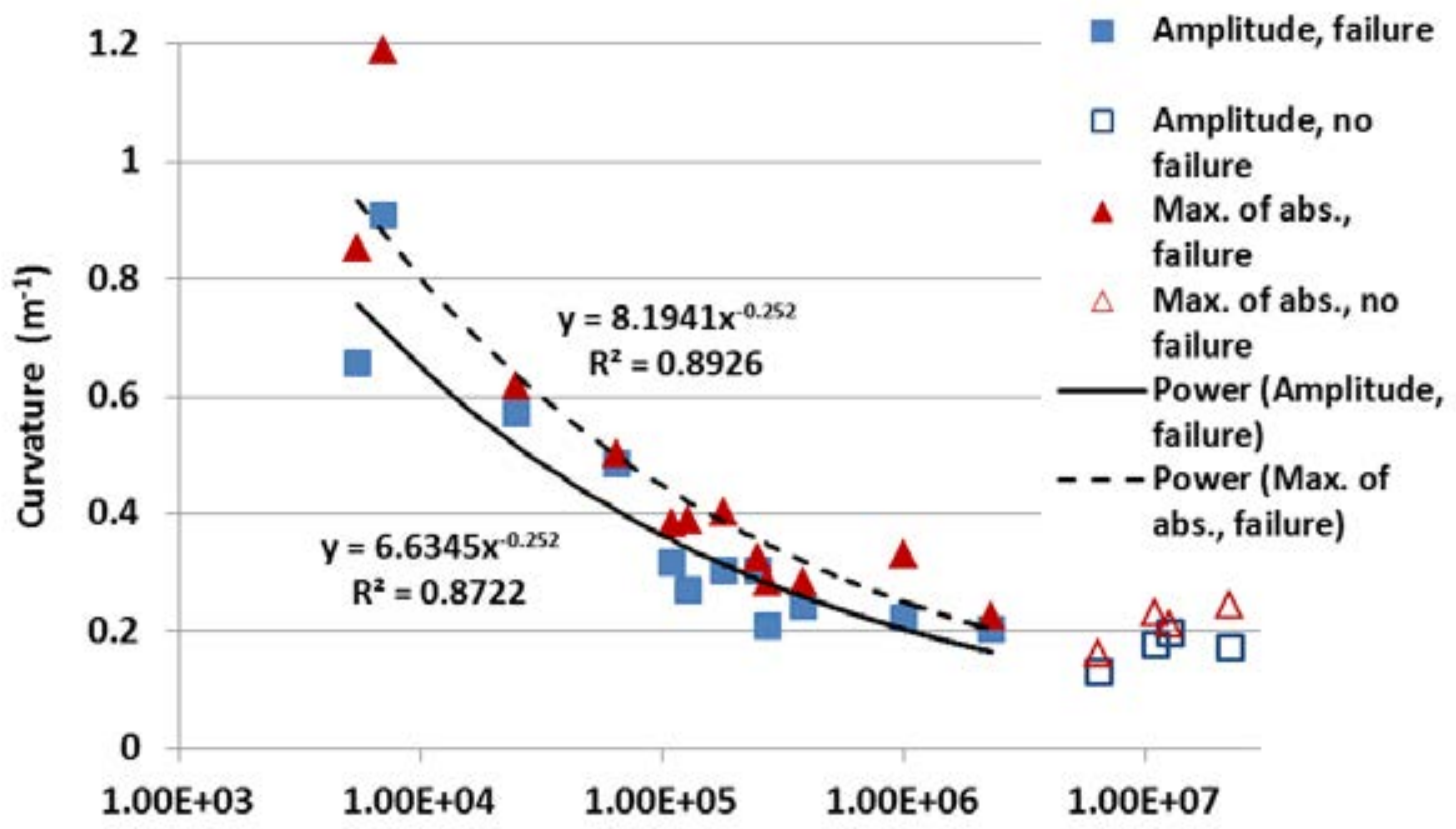

Number of Cycles or Cycles to Failure

(a)

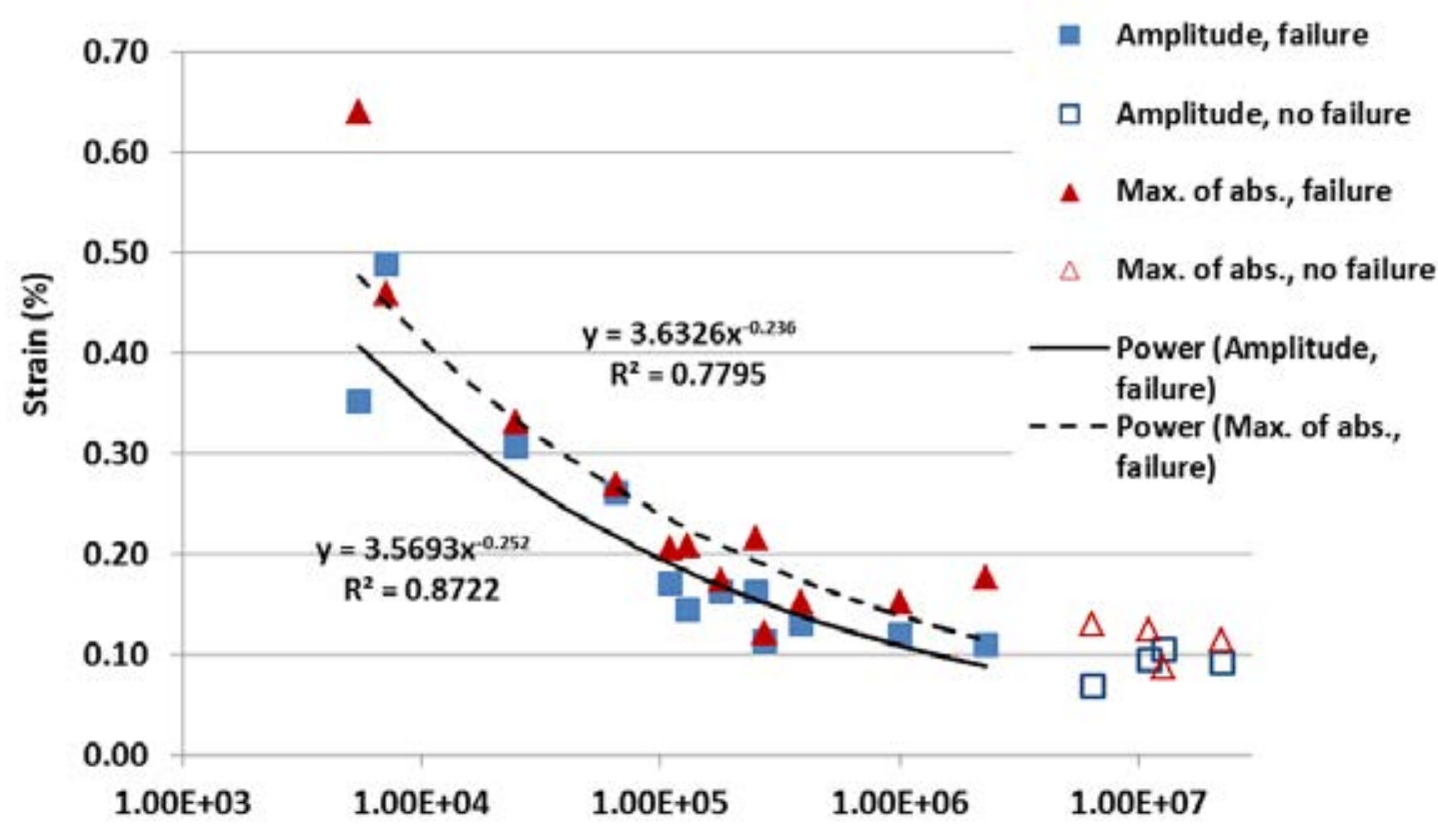

Number of Cycles or Cycles to Failure

(b)

Figure 27. (a) Maxima of absolute curvature extremes and curvature amplitudes as a function of number of cycles, (b) Maxima of absolute strain extremes and strain amplitudes as a function of number of cycles. (Solid markers represent tests with specimens failure; open markers indicate tests without failure.) 


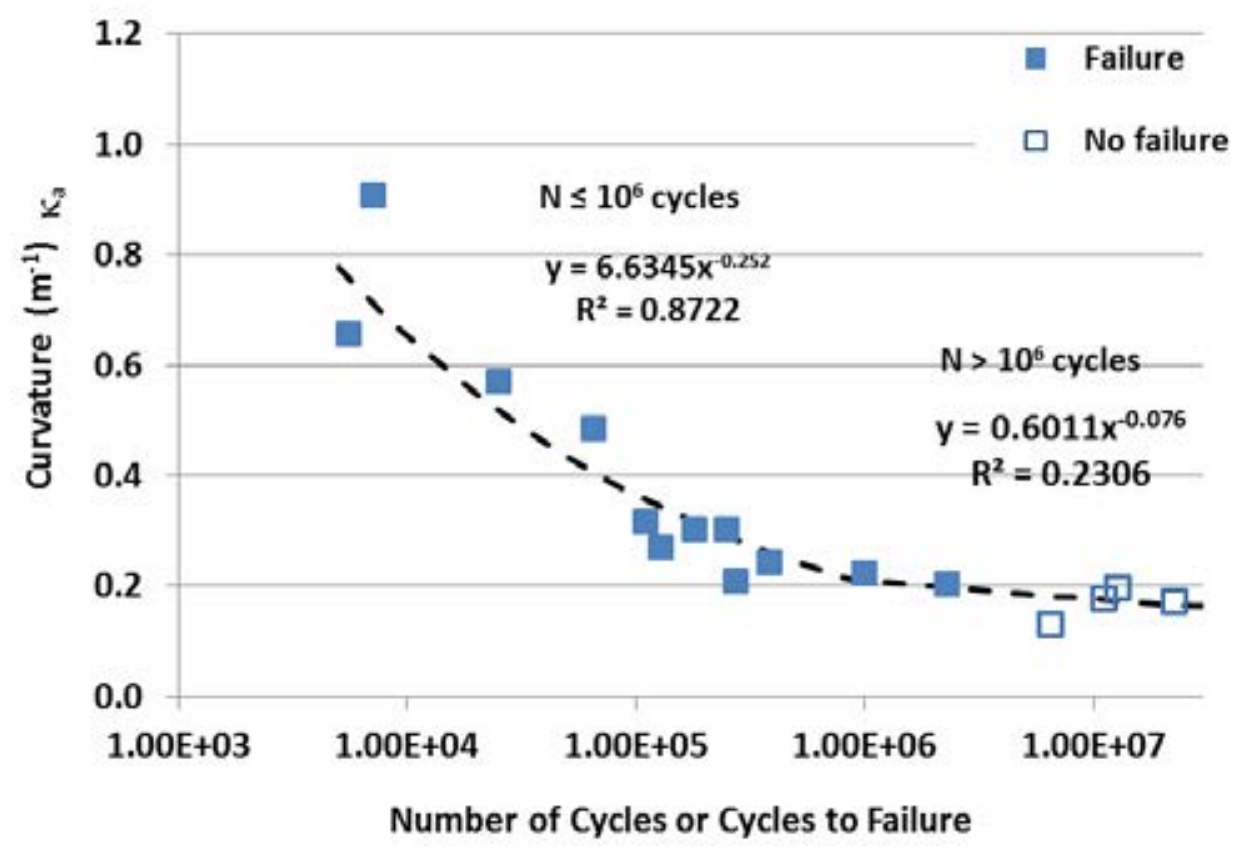

(a)

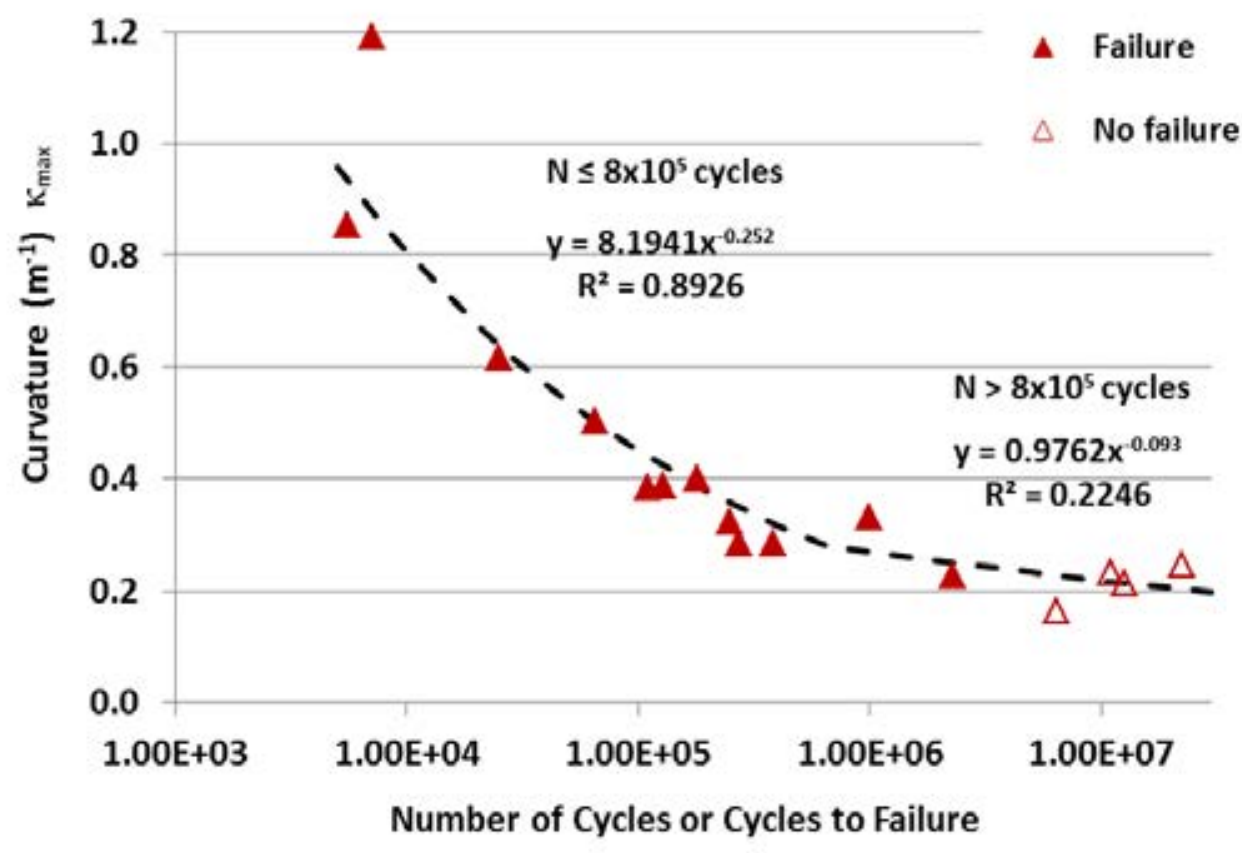

(b)

Figure 28. (a) Curvature amplitudes, (b) maxima of absolute curvature extremes, and (c) maxima of absolute strain extreme as a function of number of cycles with curve-fitting extended to include the no-failure data points. Solid markers represent tests with specimens failure; open markers indicate tests without failure. 


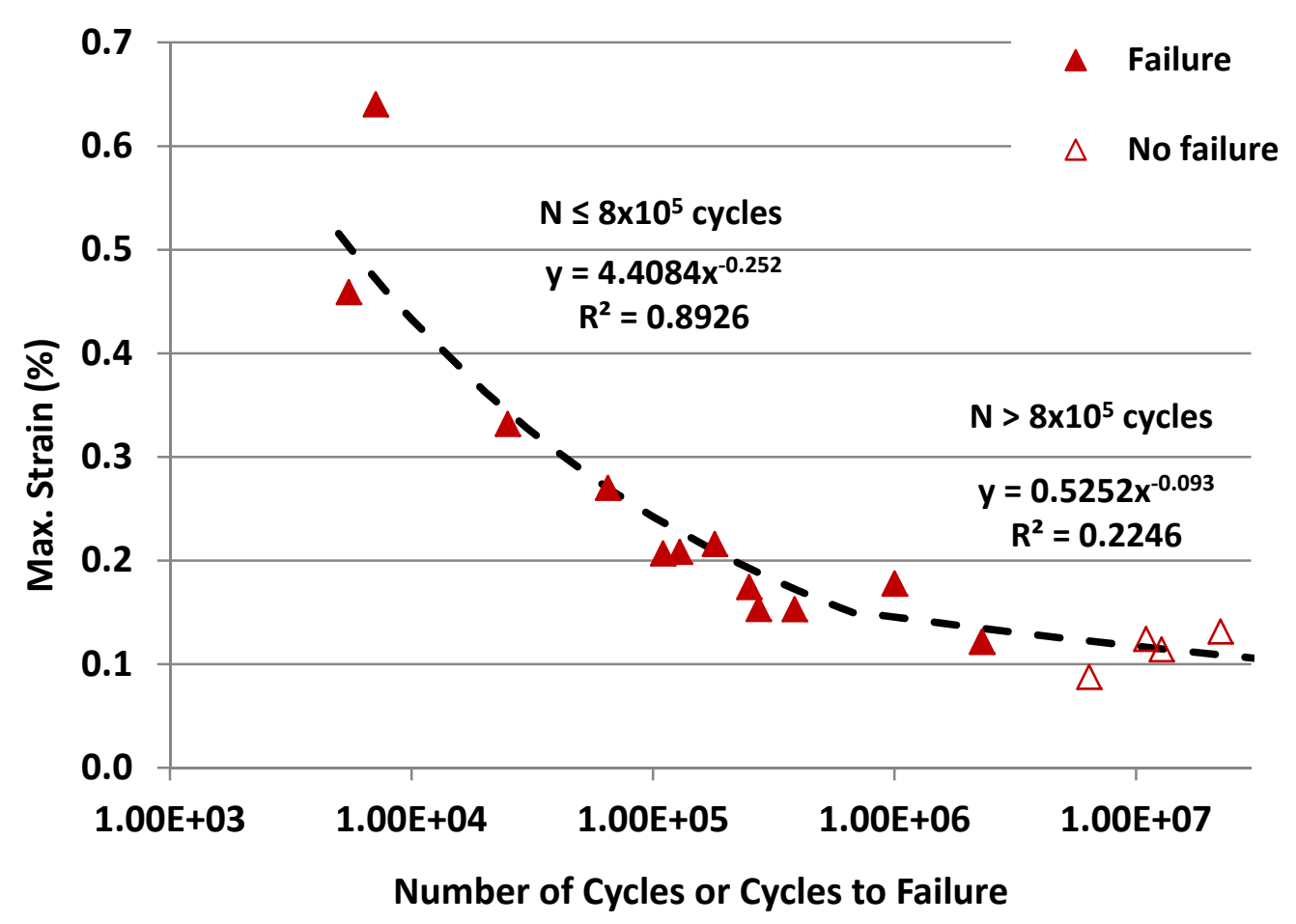

(c)

\subsubsection{Post-Irradiation Examination (PIE)}

The objective of the PIE is to gather meaningful information to identify underlying failure mechanisms. The primary focuses are:

- To generate information on cladding features such as hydrides and incipient cracks near PPIs and near the mid-pellet.

- To reveal information about the fuel/cladding bond.

- To perform PIE with the fuel pellet intact to ensure that the SNF damage mechanism associated with P-C interaction and fuel failure can be preserved at the PPI region.

PIE was conducted on five fuel segments that were taken from the same father rod (E02-605) but with different mechanical loading histories:

- Untested - 605D1D

- Tested and failed under static loading condition at a PPI - S2/ 605D1E

- Tested but non-failed under dynamic loading condition- D4/ 605D1C

- Tested and failed under dynamic loading at a PPI - D3/ 605C10A 
- Tested and failed under dynamic loading but not at PPI - D5/ 605D1B

PIEs were generally conducted on axial sections, while one PIE was carried out on a transverse section. For the tested and failed specimen, the preparation of axial sections mainly involved the following steps: (1) cut a one-inch segment on the fracture end, (2) mount the segment with the bending plane in the horizontal direction and cut the top half away, (3) back-pot the remaining half to keep all the fuel in place, and (4) grind and polish the section to the required finish for metallography. Examinations were then performed on an optical microscope. ${ }^{16}$ The section preparation for the other segments/specimens was similar to the above procedure.

PIE on the transverse section was conducted only on D3. The section was prepared using a piece of fuel rod approximately a quarter inch long cut away from the remaining half of specimen D3. The segment was defueled using hot nitric acid. The defueled cladding was removed from the hot cell and cleaned with water and alcohol in an ultrasonic cleaner. The inside and outside surfaces of the cladding were then coated with a thin layer of epoxy. The section was then ground and polished to the required finish for examination using scanning electron microscopy (SEM). Observations on the transverse section can be found in Appendix $\mathrm{F}$, and those for the axial sections will be briefly discussed in the following.

An optical image of the axial section for un-tested segment 605D is shown in Figure 29.

The three dished fuel pellets can be easily identified. Two primary fractures with sizes of pellet length and pellet radius were developed along and normal to the axial direction, respectively. Detailed study showed that the pellet-to-pellet interface near the edge of the dish and the pelletto-cladding interface were fairly close without any visible gaps. Secondary fractures had developed within pellets near the peripheral area close to the pellet-to-cladding interfaces. A cavity is also observed around some triple boundaries of pellets and cladding. The circumferential hydride layers can be clearly seen over the cladding thickness. The circumferential hydrides are widely spaced in the large middle part of the wall. The density of the hydrides increases significantly towards the outside of the cladding and in the claddingpellet interface area. The circumferential hydride layers are very sizable and, in the middle of the cladding wall, the layers can run more than $1000 \mu \mathrm{m}$. The outside surface of cladding is covered with a continuous thinner oxide layer that measured about $75 \mu \mathrm{m}$.

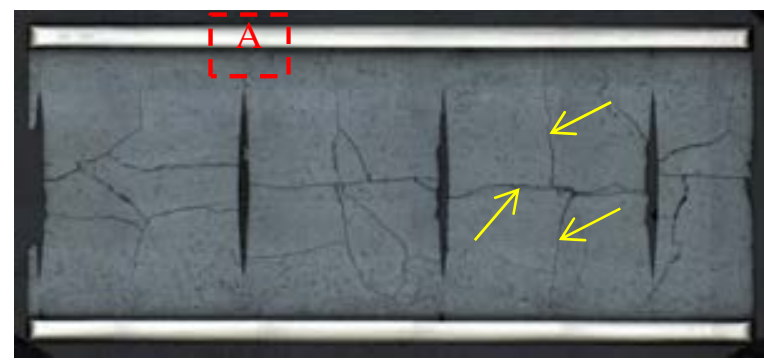

(a)

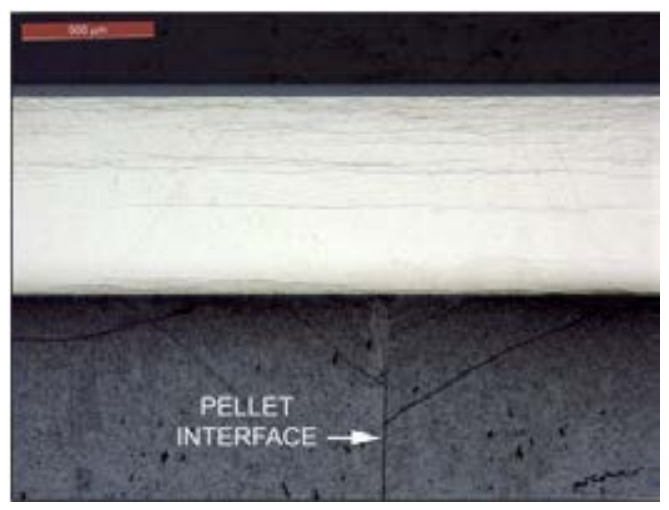

(b)

Figure 29. Optical images of untested segment 605D for (a) overall axial cross section and (b) enlarged area $A$.

Optical images based on the axial section of S2 specimen are shown in Figure 30. Fuel failure occurred at the PPI as seen from the left side images. Unlike those in the untested fuel segment, the primary axial fractures did not align but tilted toward the axial direction. 
The pellet-to-cladding interface appeared to be in good contact. The fracture surface in the cladding exhibited a zigzag pattern both on the back and front sides of the specimen. The unique pattern resulted from the combination of brittle fracture of hydrides and ductile failure of the metal matrix under tension. Delamination can be seen over the hydride layers near the fracture surface. In addition, spalling and cracking of the surface oxide layer can be seen on the back and front sides.

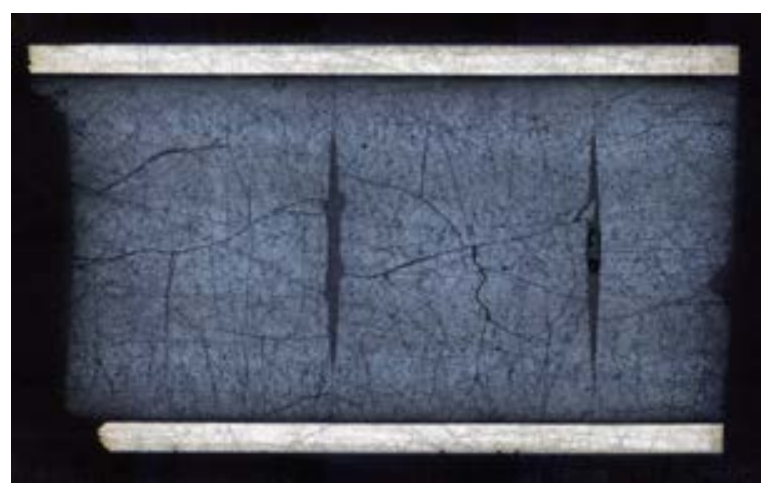

(a)
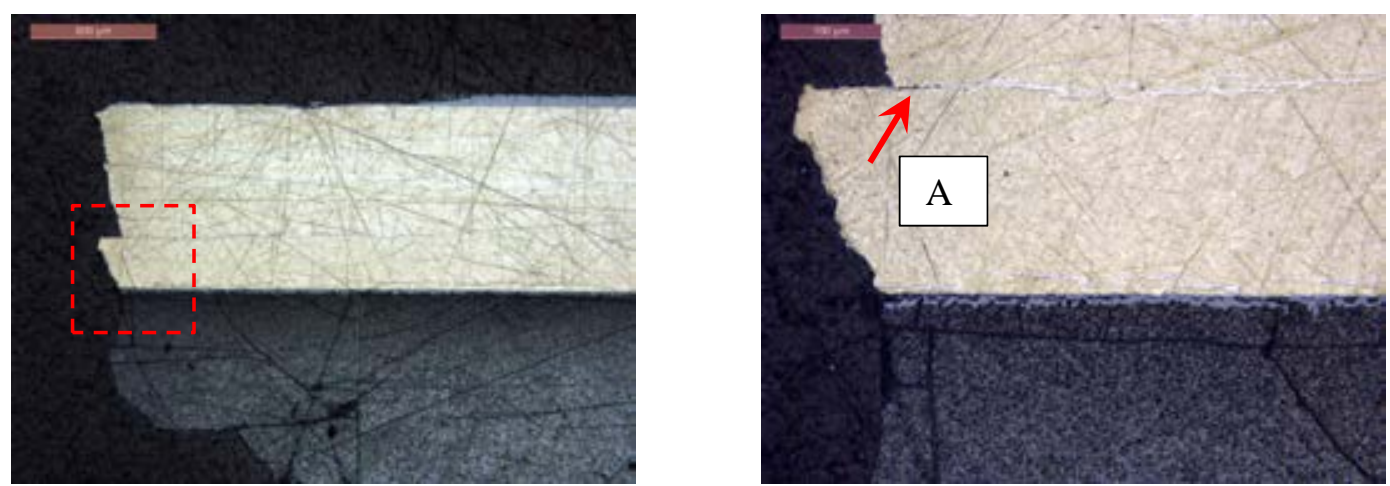

(b)
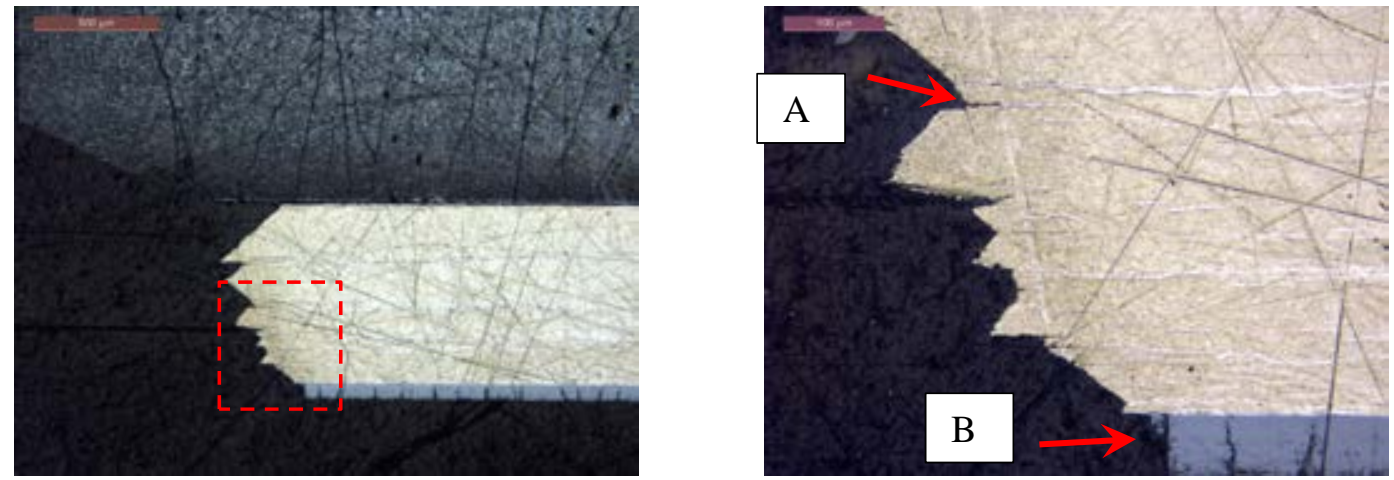

(c)

Figure 30. (a) Axial cross section of S2/ 605D1E, (b) fracture surface near back side of rod and an enlarged area, and (c) fracture surface near front side of rod and an enlarged area. A - delamination, B - cracking of oxide layer. 
PIEs of the other fuel segments can be found in Appendix F, including five axial sections and one transverse section.

Some technical difficulty was encountered when preparing the PIE polished specimens. The foreign particles from the polished specimen (such as fine fuel particle due to possible micro fracturing of the fuel during dynamic testing) were easily brought into the working surface, introducing scratches.

The following observations were made based on the PIE.

- PPIs and PCls near the edges or peripheral area of pellets appeared to be in good contact. No significant gap or filling was observed in the interfaces.

- The pellets exhibited fracturing that was normal for irradiated fuel. These fractures were observed in both untested and tested fuel segments.

- Specimen failures occurred primarily at PPI.

- The density of the hydride layers increases significantly toward the outside of cladding wall. The cladding outer wall was covered with an oxide layer of $75 \mu \mathrm{m}$ thick. 


\section{CONCLUSIONS}

High burnup H. B. Robinson fuel rods were tested and evaluated under both static and dynamic loading conditions using the CIRFT equipment developed at ORNL. The CIRFT system is composed of a $U$-frame equipped with load cells for imposing the pure bending loads on the spent fuel rod test specimen and measuring the in-situ curvature of the fuel rod during bending using a 3-LVDTs set-up. In general, the static CIRFT test results indicate a significant increase in flexural rigidity compared to that of the defueled HBU rod specimen. Nevertheless, the segment composite structure of an HBU rod also introduces numerous stress concentration sites into an HBU rod system, ultimately resulting in HBU specimen fractured at the pellet-pellet interface regions under dynamic CIRFT fatigue testing.

The static tests were conducted under displacement control at a rate of $0.1 \mathrm{~mm} / \mathrm{s}$ to a maximum displacement of $12.0 \mathrm{~mm}$ at each loading point. Dynamic tests were conducted under load control of \pm 5.08 to $\pm 35.56 \mathrm{~N} \cdot \mathrm{m}, 5 \mathrm{~Hz}$. PIEs were performed on selected specimens. The following conclusions can be drawn:

- $\quad$ The HBU HBR rods survived static unidirectional bending to a maximum curvature of 3.1 to $3.5 \mathrm{~m}^{-1}$ or a maximum moment of 86 to $87 \mathrm{~N} \cdot \mathrm{m}$ (Table 4). The maximum equivalent strain was 1.7 to $1.9 \%$, corresponding to an equivalent stress of 733 to $748 \mathrm{MPa}$ (Table 5).

- The HBU HBR rods exhibited a multiple-stage constitutive response with two linear stages followed by a nonlinear stage. The flexural rigidity at the initial stage was 52 to $63 \mathrm{~N} \cdot \mathrm{m}^{2}$, corresponding to an elastic modulus of 83 to $101 \mathrm{GPa}$ (Table 5).

- The equivalent $0.2 \%$ yield strength of the $\mathrm{HBU}$ cladding was estimated at 687 to $727 \mathrm{MPa}$ (Table 5), depending on the level of hydrogen content or magnitude of oxidation thickness.

- A large majority of the specimen failures of the HBU HBR rods under static unidirectional loading occurred near the PPI as validated by the post-test or PIEs.

- The fatigue life of HBU HBR rods in the cyclic test depended on the level of loading. Under loading with moments of \pm 8.89 to $\pm 35.56 \mathrm{~N} \cdot \mathrm{m}$, and strain of \pm 0.07 to $\pm 0.49 \%$ at $5 \mathrm{~Hz}$ (Table 7 ), the fatigue life ranged from $5.5 \times 10^{3}$ to $2.3 \times 10^{6}$ cycles.

- The $\varepsilon-\mathrm{N}$ curve of the HBU HBR rods can be described by a power function of $y=$ 3.5693 $x^{-0.252}$, where $x$ is the number of cycles to failure, and $y$ is the strain amplitude (\%).

- Based on strain amplitude, a fatigue limit is likely located between 0.106 and $0.110 \%$ if it is defined at $10^{7}$ cycles.

- Maxima of the imposed curvature in dynamic tests ranged from \pm 0.16 to $\pm 1.19 \mathrm{~m}^{-1}$ at $5 \mathrm{~Hz}$ (Table 7). The $\kappa-\mathrm{N}$ curve of the HBU HBR rods can be described by a power function of $\mathrm{y}=$ $8.1941 \cdot \mathrm{x}^{-0.252}$, where $x$ is the number of cycles to failure and $y$ is the maxima of cladding tensile curvature $|\kappa| \max \left(\mathrm{m}^{-1}\right)$. A fatigue limit is likely located between 0.226 and $0.245 \mathrm{~m}^{-1}$ if it is defined at $10^{7}$ cycles. 
- The failure of HBU HBR rods under cyclic reverse loading was primarily due to pelletcladding interaction and generally resided at the pellet-to-pellet interface, as validated by the PIE. 


\section{REFERENCES}

1. Design, Operation, and Performance Data for High Burnup PWR Fuel from H. B. Robinson Plant for Use in the NRC Experimental Program at Argonne National Laboratory. EPRI, Palo Alto, CA: 2001.1001558

2. B. Hanson et al., Gap Analysis to Support Extended Storage of Used Nuclear Fuel Rev. 0. U.S. Department of Energy, Washington, DC: January 2012.

3. J.-A. J. Wang et al., High Burn-up Spent Fuel Vibration Integrity Study Progress Letter Report (Out-of-Cell Fatigue Testing Development-Task 2.1). Oak Ridge National Laboratory, Oak Ridge, TN: January 2011. ORNL/TM-2010/288

4. J.-A. J. Wang, et al., Progress Letter Report on U Frame Test Setup and Bending Fatigue Test for Vibration Integrity Study (Out-of-Cell Fatigue Testing Development-Task 2.2). Oak Ridge National Laboratory, Oak Ridge, TN: January 2012. ORNL/TM-2011/531

5. J.-A. J. Wang, et al., Progress Letter Report on U-Frame Test Setup and Bending Fatigue Test for Vibration Integrity Study (Out-of-Cell Fatigue Testing Development-Task 2.3." Oak Ridge National Laboratory, Oak Ridge, TN: August 2012. ORNL/TM-2012/417

6. J.-A. J. Wang, et al., An Innovative Dynamic Reversal Bending Fatigue Testing System for Evaluating Spent Nuclear Fuel Rod Vibration Integrity or Other Materials Fatigue Aging Performance, ORNL Invention Disclosure 201102593, DOE S 124,149, Oak Ridge National Laboratory, Oak Ridge, TN: April 2011. Patent in review, 13/396,413: February 2012.

7. H. Wang, et al., Development of U-frame Bending System for Studying the Vibration Integrity of Spent Nuclear Fuel. Journal of Nuclear Materials: 2013. 440, 201-213

8. J.-A. J. Wang et al., SNF Test System for Bending Stiffness and Vibration Integrity," International High-Level. Radioactive Waste Management Conference, Albuquerque, NM: April 2013.

9. J.-A. J. Wang, et al., Progress Letter Report on Bending Fatigue Test System Development for Spent Nuclear Fuel Vibration Integrity Study (Out-of-Cell Fatigue Testing Development-Task 2.4). Oak Ridge National Laboratory, Oak Ridge, TN: July 2013. ORNL/TM-2013/225

10. J.-A. J. Wang, et al., Reversible Bending Fatigue Test System for Investigating Vibration Integrity of Spent Nuclear Fuel During Transportation," $17^{\text {th }}$ International Symposium on the Packaging and Transportation of Radioactive Materials (PATRAM 2013), San Francisco, CA: August 2013.

11. J.-A. J. Wang and H. Wang, Progress Letter Report on Reversal Bending Fatigue Testing of Zry-4 Surrogate Rod (Out-of-Cell Fatigue Testing Development-Task 2.4). Oak Ridge National Laboratory, Oak Ridge, TN: August 2013. ORNL/TM-2013/297

12. G. Bjorkman, High Burnup Spent Fuel Testing Program Objectives. NRC Program Review Meeting, Oak Ridge, TN: August 2011.

13. J.-A. J. Wang and H. Wang, 2014 Semi-Annual Progress Letter Report on Used Nuclear Fuel Integrity Study in Transportation Environments. Oak Ridge National Laboratory, Oak Ridge, TN: April 2014. ORNL/TM-2014/63 
14. C. Baldwin, Oak Ridge National Laboratory, Mar. 6, 2013. Reproduced in Appendix B.

15. Cladding Embrittlement during Postulated Loss-of coolant Accidents. U.S. Nuclear Regulatory Commission, Office of Nuclear Regulatory Research, Rockville, MD: July 2008. NUREG/CR-6967/ANL-07/04

16. M.C. Billone, et al., Baseline Properties and DBTT of High-Burnup PWR Cladding Alloys," $17^{\text {th }}$ International Symposium on the Packaging and Transportation of Radioactive Materials (PATRAM 2013), San Francisco, CA: August 2013.

17. J-A Wang, H. Jiang, "Quantification of CIRFT System Biases and Uncertainties When Testing High-Burnup Spent Nuclear Fuel. Oak Ridge National Laboratory, Oak Ridge, TN: September 2014. DOE FCRFD-2014-000604 and ORNL/TM-2014/288

18. R.S. Daum, et al., Experimental and analytical investigation of the mechanical behavior of high-burnup Zircaloy-4 fuel cladding. Journal of ASTM International. Paper ID JAI101209.

19. J-A Wang, et al., Using Finite Model Analysis and Out of Hot Cell Surrogate Rod Testing to Analyze High Burnup Used Nuclear Fuel Mechanical Properties. Oak Ridge National Laboratory, Oak Ridge, TN: August 2014. ORNL/TM-2014/257 and DOE FCRD-UFD-2014000603, 
APPENDIX A

ROD SEGMENTS AND CUTTING PLAN 



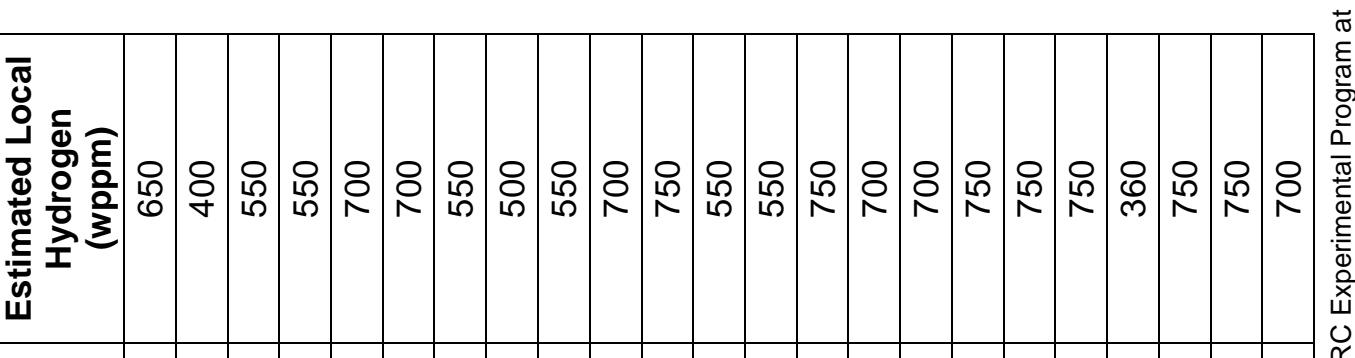

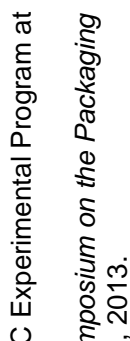

늘

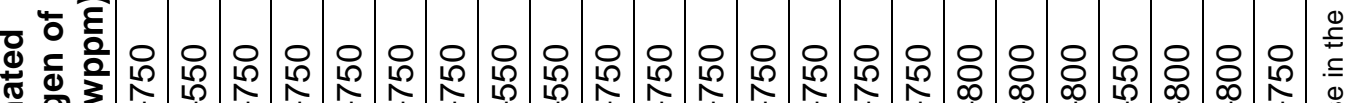

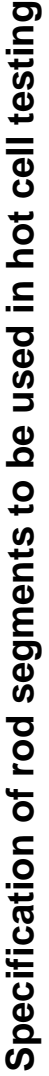

E

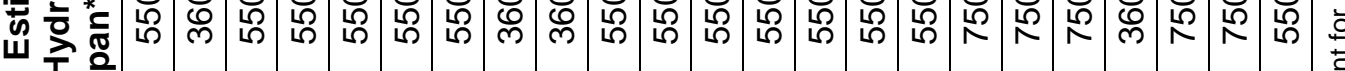

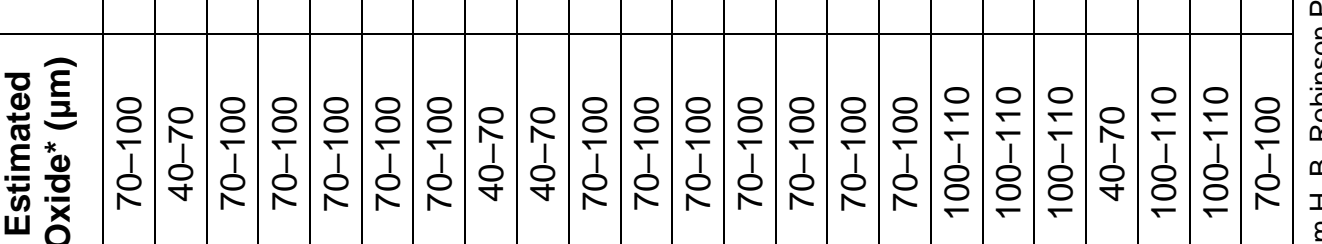

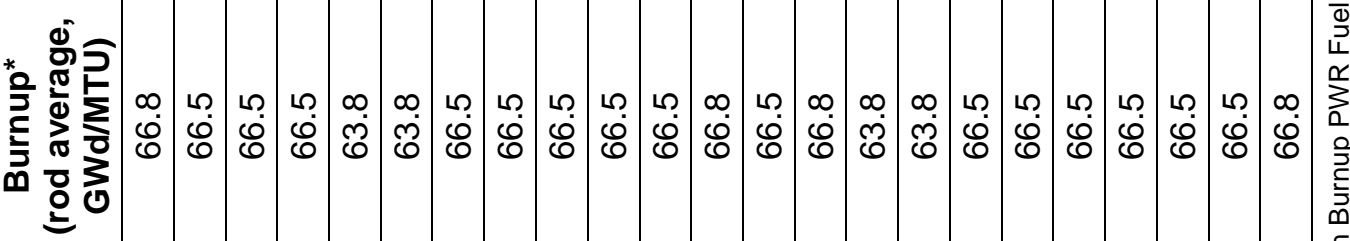

(4)

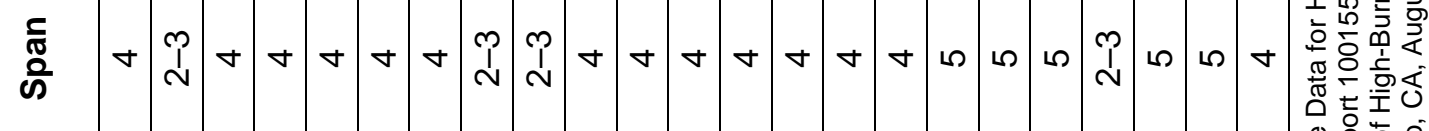

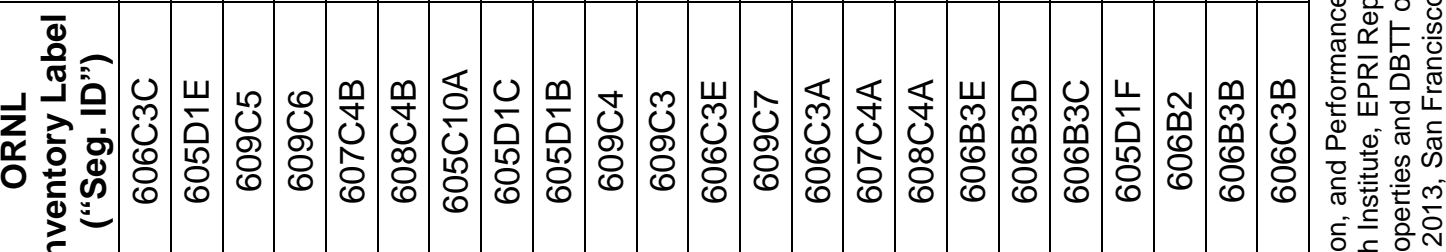

$ه$ 응

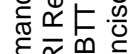

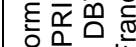

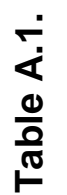

లే ब ब

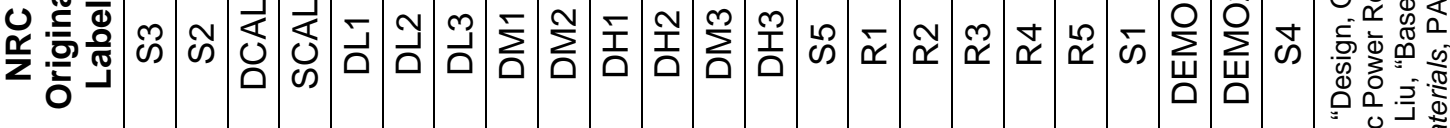

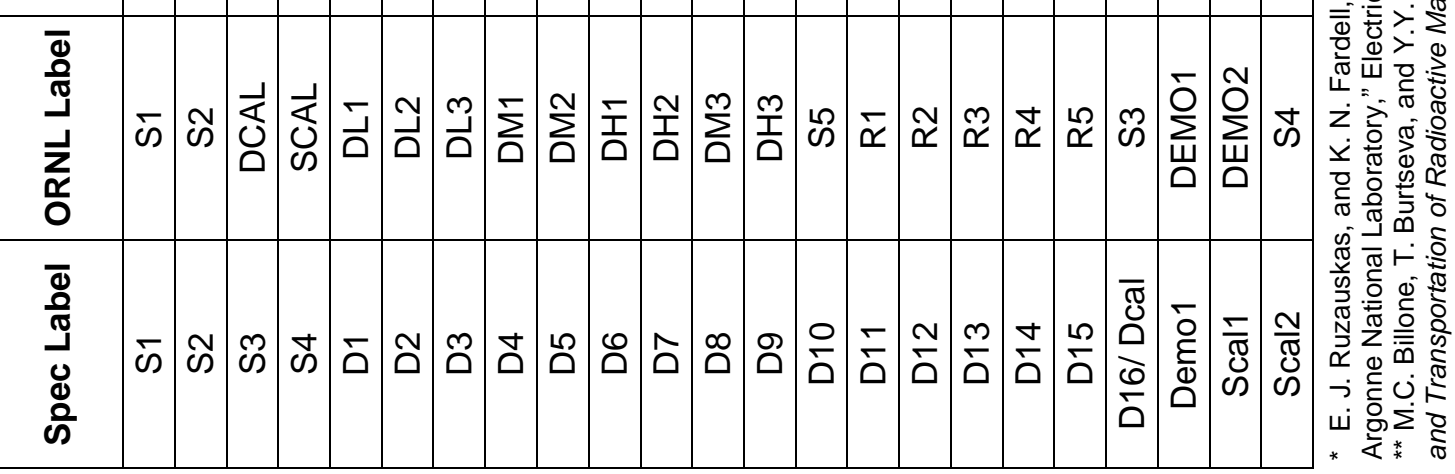




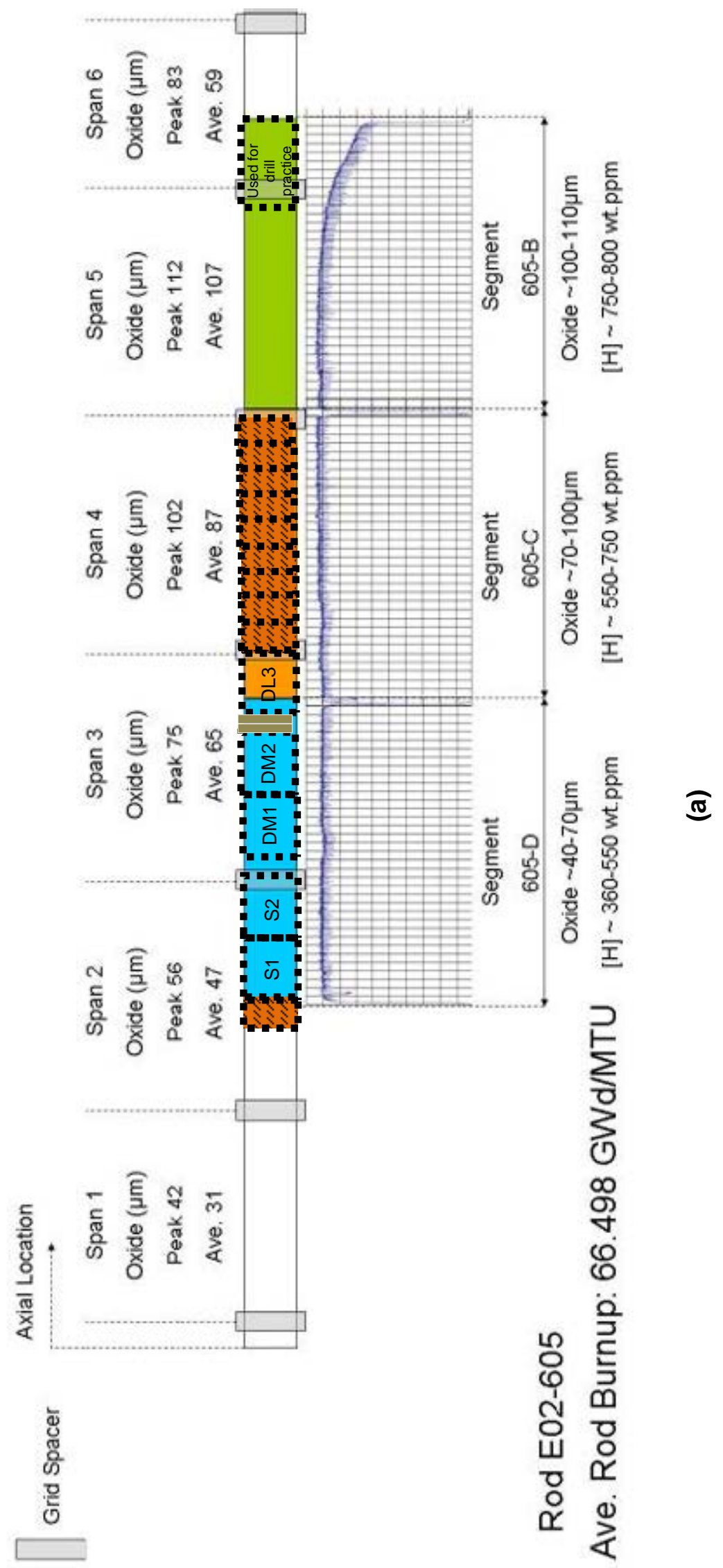




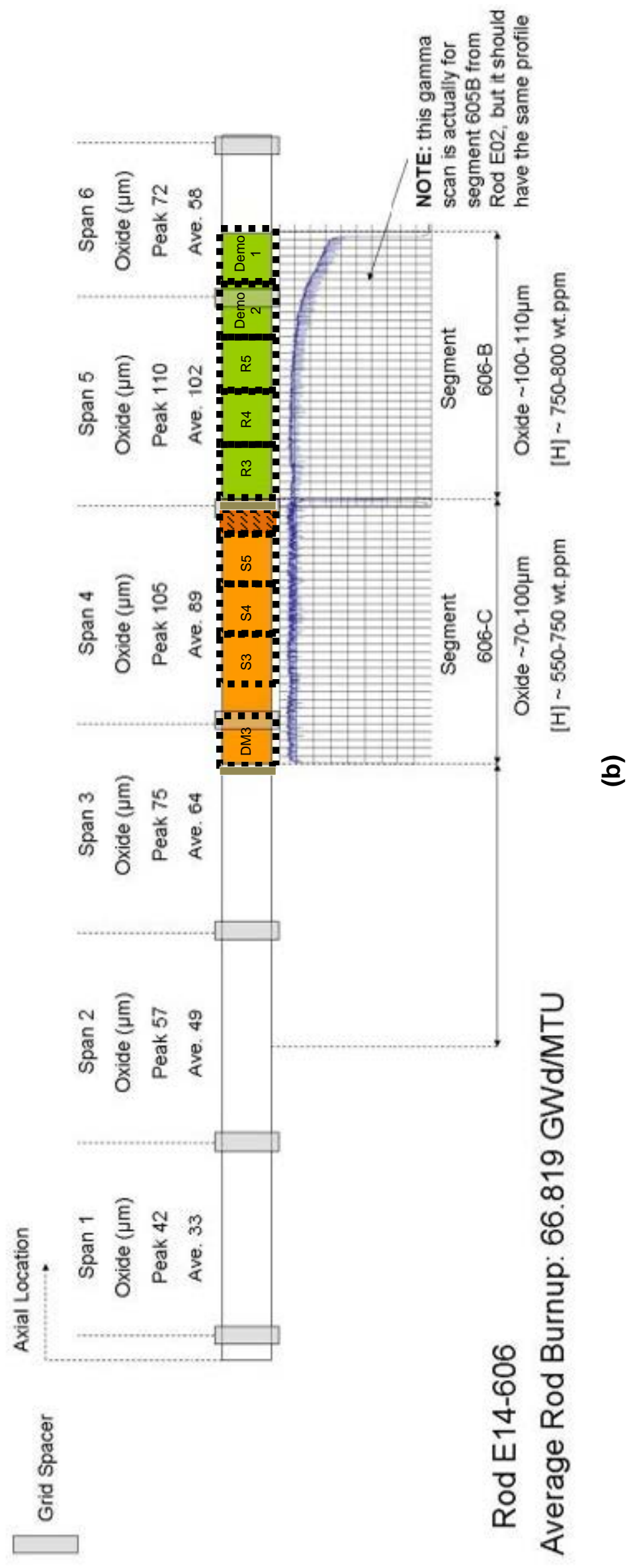

A-3 

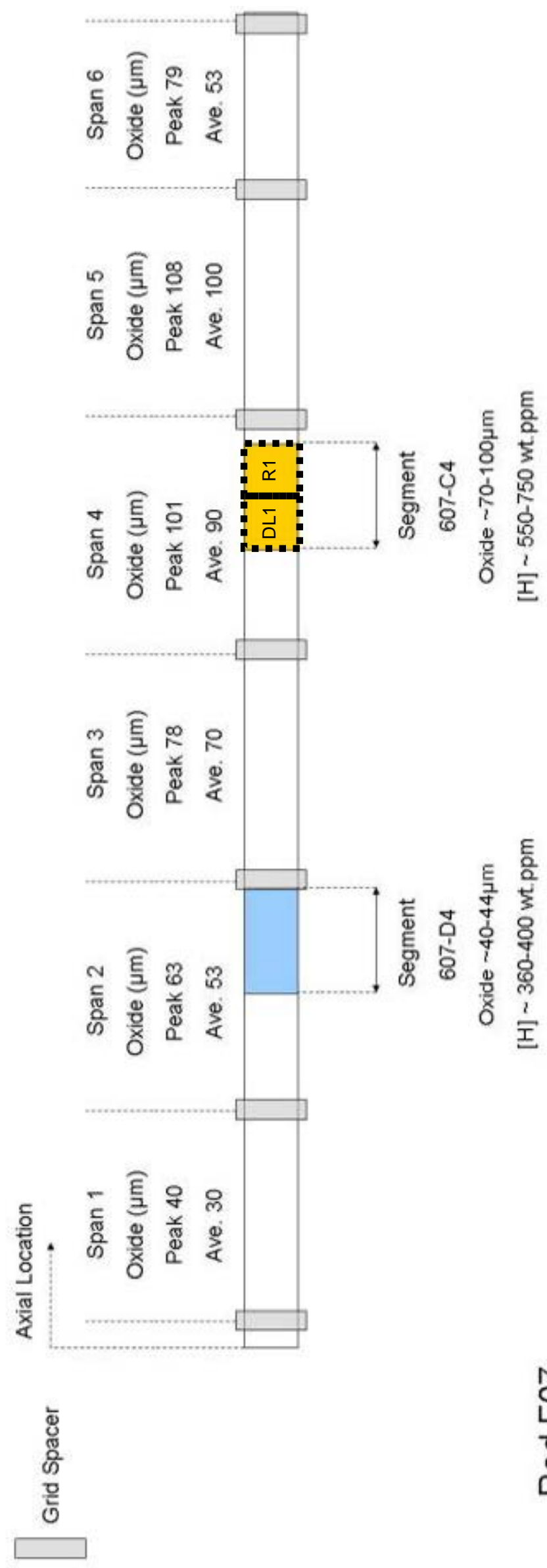

0

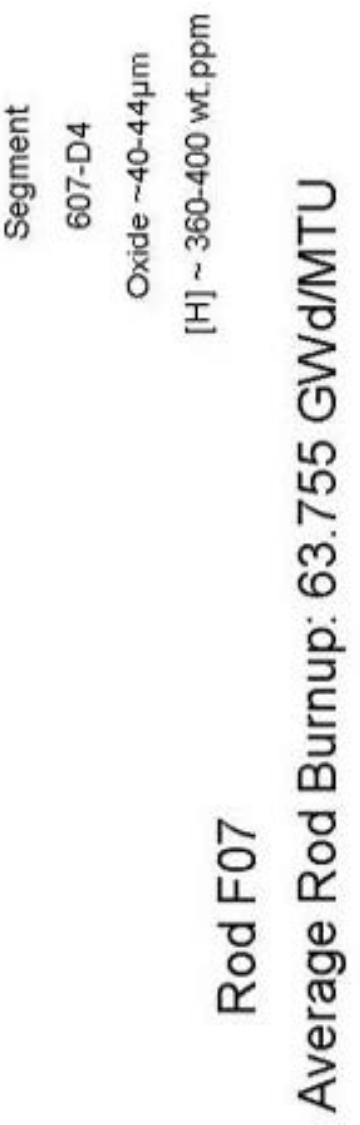




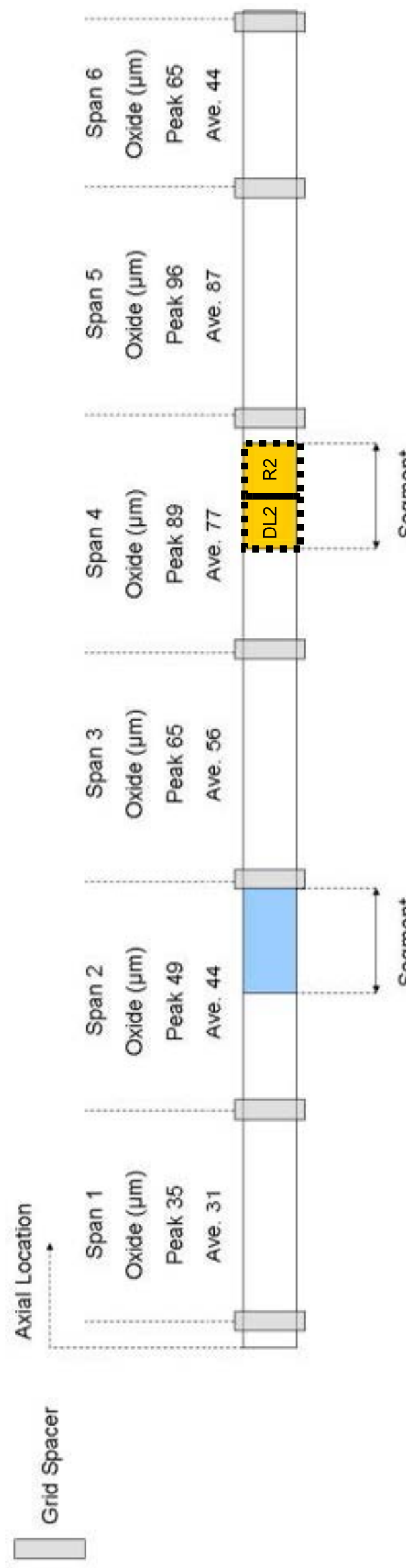

ฮิ

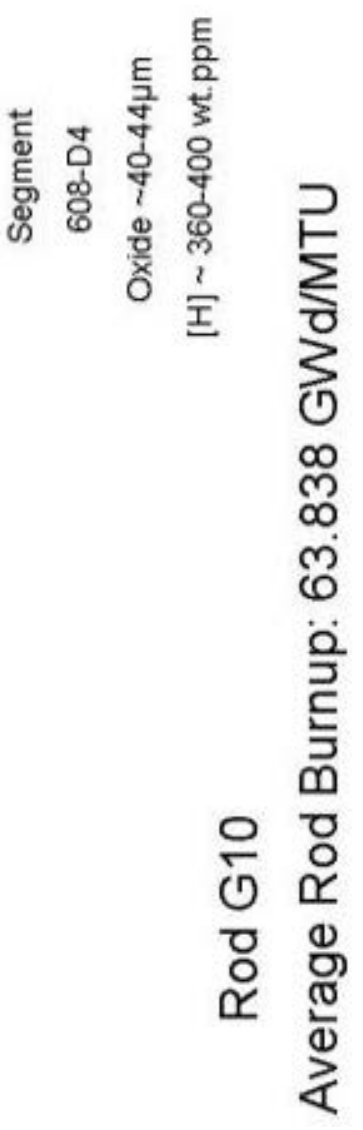

A-5 


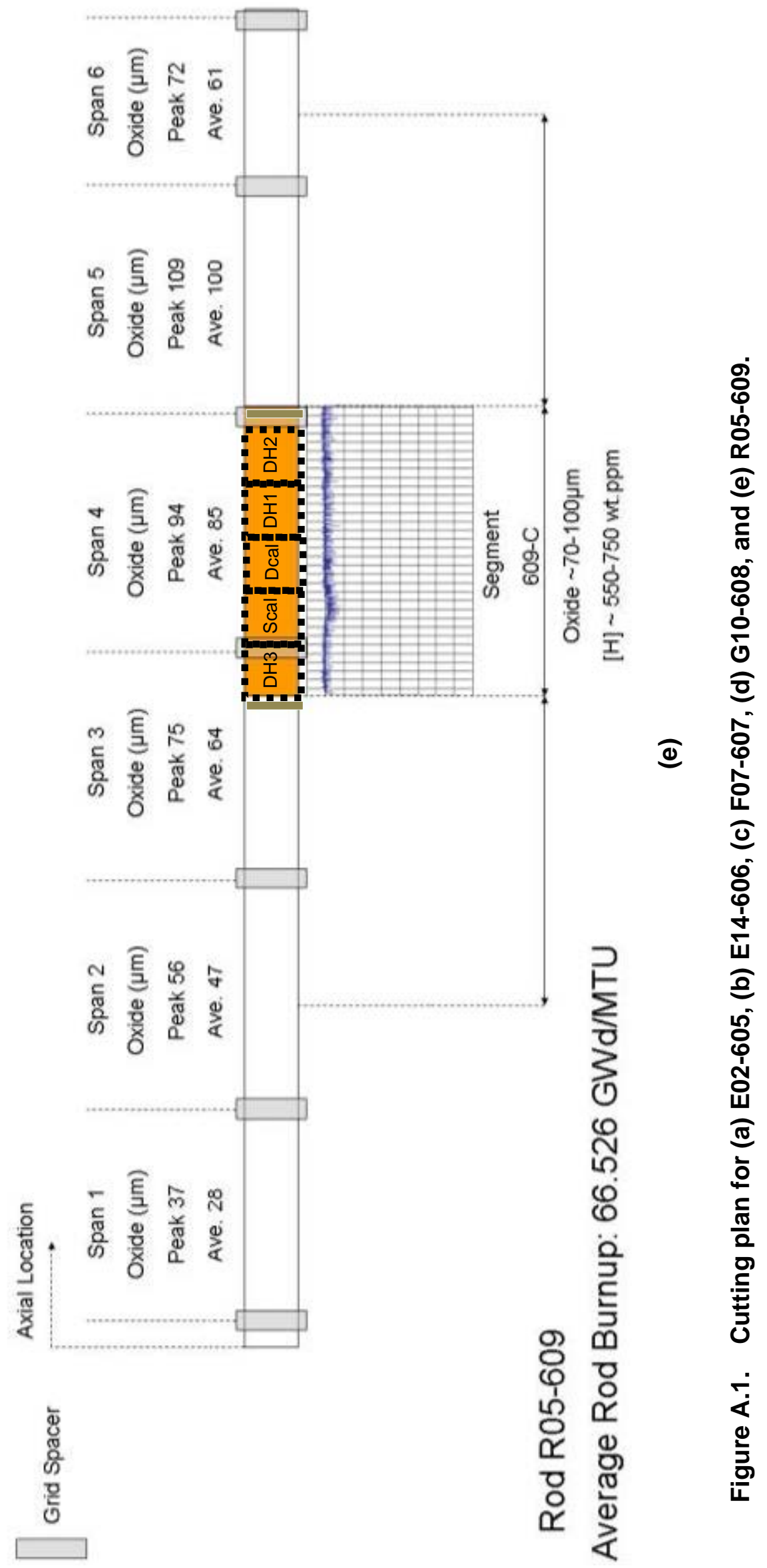

A-6 
APPENDIX B

\section{METROLOGY OF ROD SEGMENTS}





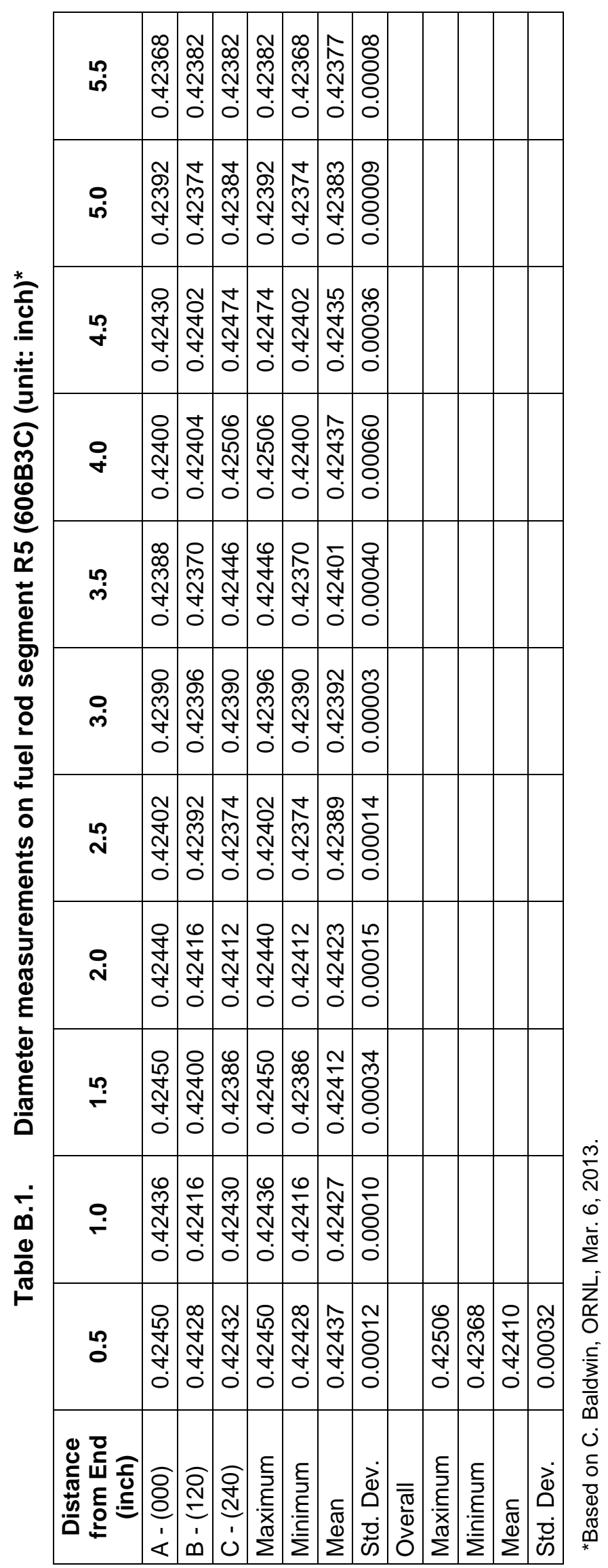




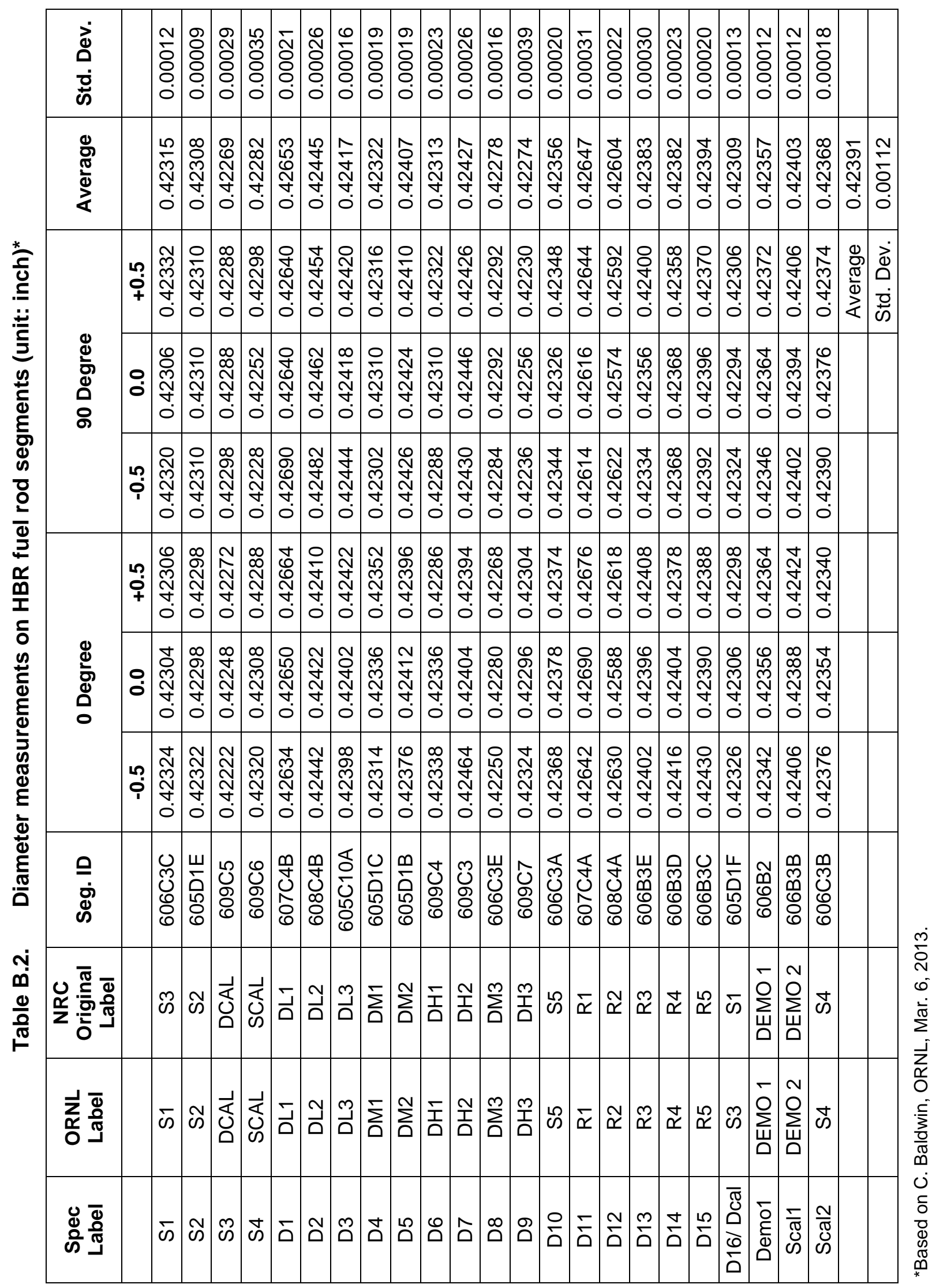


APPENDIX C

BENCHMARKING OF TEST SYSTEM 



\section{TUNING AND STATIC TEST ON DEMO1/ 606B2 (+ DYNAMIC)}

Tuning was completed September 23, 2013, on test specimen Demo1 (606B2) by using TunelQ on both motors (Table C.1).

Table C.1. Tuning parameters obtained for test specimen Demo1

\begin{tabular}{|c|c|c|c|}
\hline \multicolumn{2}{|c|}{ Axial 1 } & \multicolumn{2}{c|}{ Axial 2 } \\
\hline TunelQ1 & 1.5860 & TunelQ1 & 1.8869 \\
\hline TunelQ2 & 0.0192 & TunelQ2 & 0.0193 \\
\hline TunelQ3 & -9.6663 & TunelQ3 & -11.0162 \\
\hline
\end{tabular}

Demo1 (606B2, 66.5 GWd/MTU burnup, 100-110 $\mu \mathrm{m}$ oxide layer, 750 ppm H content) was initially tested to the relative displacement of $2 \mathrm{~mm}$, at rate $0.1 \mathrm{~mm} / \mathrm{s}$ at each loading point on the U-frame. Such a displacement level was quite low so as to serve as a pilot test to detect the response of the rod. The rod was then tested for 11 cycles with an increased relative displacement of $20 \mathrm{~mm}$, corresponding to a moment of $77.7 \mathrm{Nm}$ (load correction applied regarding the resistive force from testing system, same applies in the following unless specified otherwise), and the rod appeared to be quite stable. Four additional cycles were carried out with further increased displacement of $24 \mathrm{~mm}$ (moment $85 \mathrm{~N} \cdot \mathrm{m}$ ), and the rod was found to still work fine. The responses of the rod during the above loading processes are given in Figure C.1.

A follow-up cycle test was conducted by using $\pm 300 \mathrm{~N}$ or $30.48 \mathrm{~N} \cdot \mathrm{m} 5 \mathrm{~Hz}$. The rod eventually fractured in the gage section near motor 1 around $3.96 \times 10^{3}$ cycles. The monitored curvature, moment, and deduced flexural rigidity as a function of number of cycles are given in Figure C.2. The fracture surfaces (Figure C.3) of the rod indicate that the failure likely involved the fracture of a fuel pellet near the interface as can be seen from the attached fragments.

It has been observed that at a low displacement level, the rod exhibited a linear response. A flexural rigidity of $40 \mathrm{~N} \cdot \mathrm{m}^{2}$ was obtained from the slope of the curvature-moment curve. A nonlinear response was exhibited under increased displacement. Three deformation stages can be delineated with two turning points near 20 and $55 \mathrm{~N} \cdot \mathrm{m}$, at which the slope of the curve changed. Below the first turning point, the response appeared repeatable under loading. Beyond the second turning point, the response of the rod became nonlinear. Unloading -loading curves were mostly overlaid if the loading level was less than the previous maximum level; otherwise, the loading curve followed the response of the rod in a monotonic loading process.

It thus can be seen from testing of the first fuel rod that:

- $\quad$ The testing system worked stably for both static and dynamic loadings when the tuning parameters generated above were used.

- A three-stage deformation process was evident when the deformation was substantially large.

- No failure was seen from use of Demo1 under static loading, even though the displacement imposed almost reached the system capacity $(25 \mathrm{~mm})$. Therefore, a cyclic loading process is required to observe the failure mode of the rod.

- The testing setup worked as designed, confining the final failure within the gage section of the rod. 


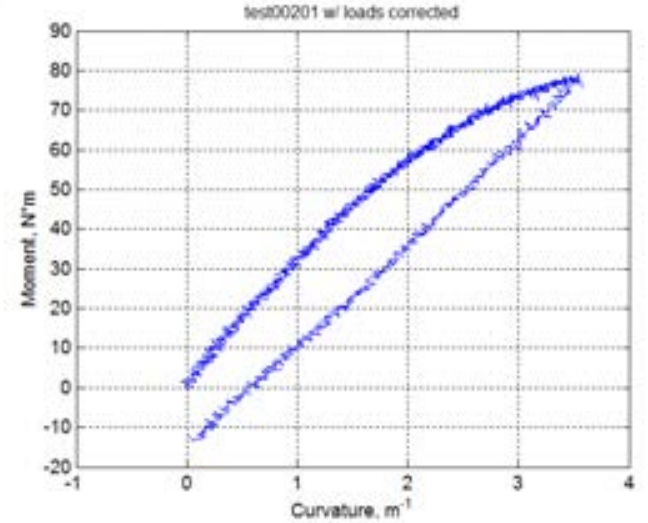

(a)

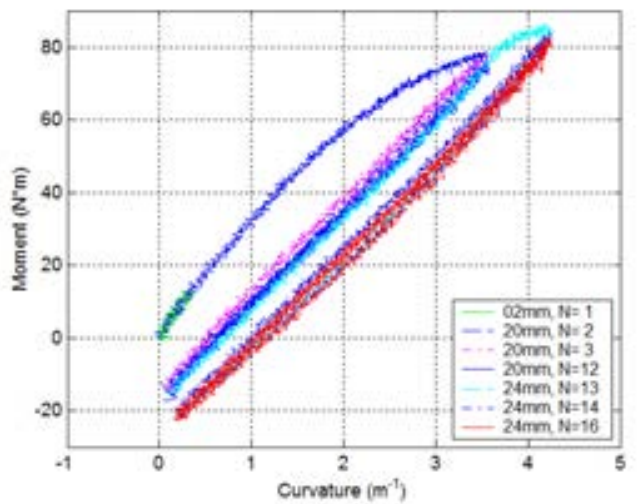

(b)

Figure C.1. (a) Curve of moment versus curvature with maximum relative displacement of $20 \mathrm{~mm}$, and (b) moment versus curvature in the subsequent repeats of static tests with relative displacement of 20 or $24 \mathrm{~mm}$ for Demo1 (606B2). The rate at the U-frame loading point was set at 0.1 and $0.2 \mathrm{~mm} / \mathrm{s}$ for loading and unloading, respectively.

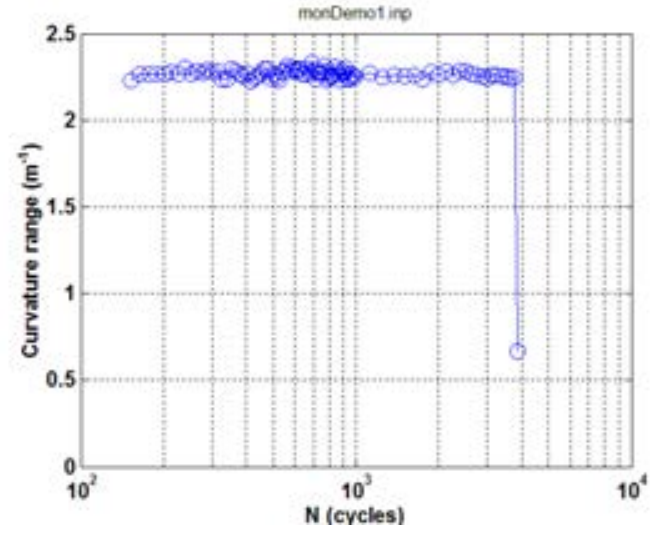

(a)

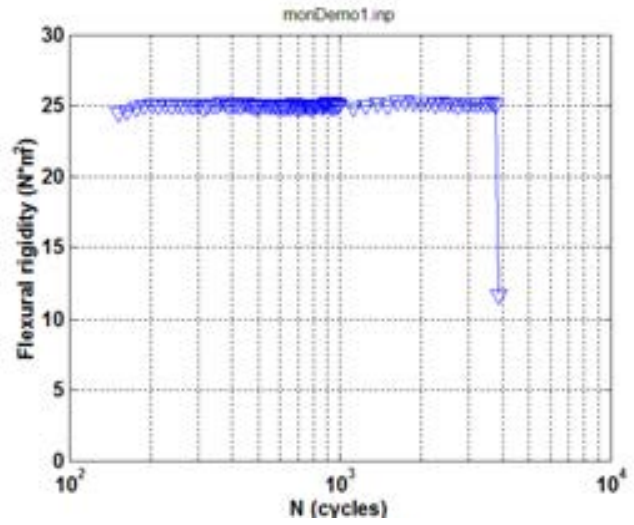

(c)

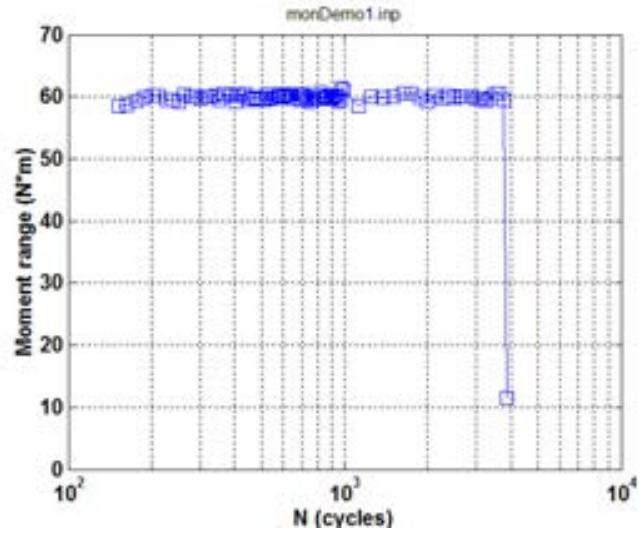

(b)

Figure C.2. Variations in (a) curvature range, (b) applied moment range, and (c) flexural rigidity as a function of number of cycles for Demo1 (606B2); $N_{f}=3.96 \times 10^{3}$ cycles under $\pm 30.48 \mathrm{~N} \cdot \mathbf{m} 5 \mathrm{~Hz}$. The specimen was subjected to repeats of static loading before the cycle test. 


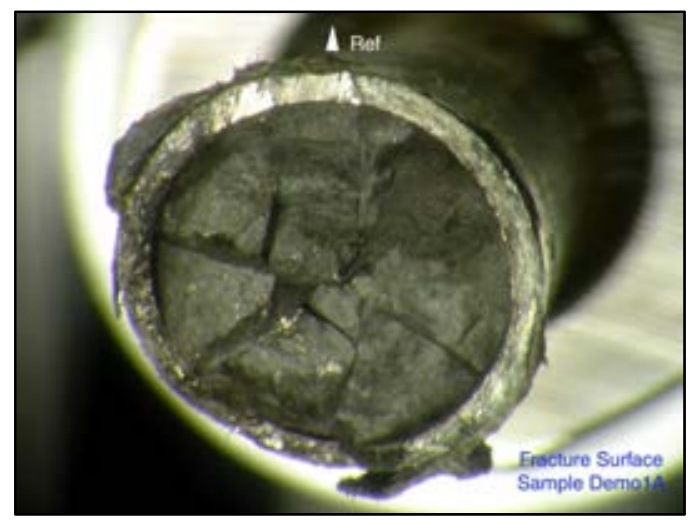

(a)

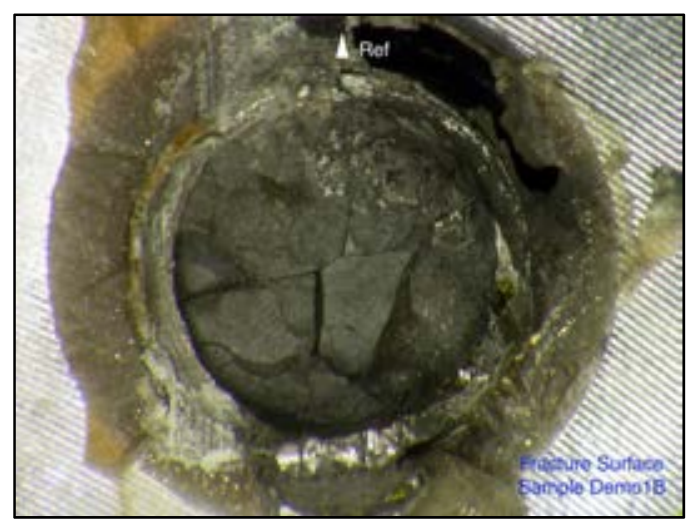

(b)

Figure C.3. Mating fracture surfaces of Demo1 (606B2); the reference marker indicates the frontal maximum stress line (facing the operator); $N_{f}=3.96 \times 10^{3}$ cycles under \pm 30.48 $\mathrm{N} \cdot \mathrm{m} 5 \mathrm{~Hz}$. The specimen had been subjected to repeat static loading cycles before the dynamic cycle test.

\section{STATIC TEST ON SCAL1/ DEMO2/ 606B3B}

The test on Scal1/ Demo2 (606B3B, 66.5 GWd/MTU burnup, 100-110 $\mu \mathrm{m}$ oxide layer, 750 ppm $\mathrm{H}$ content) was conducted at $0.1 \mathrm{~mm} / \mathrm{s}$ at each loading point of the $U$-frame. The rod fractured when the relative displacement reached $22.920 \mathrm{~mm}$, corresponding to a fracture moment of $78.2 \mathrm{~N} \cdot \mathrm{m}$. Again, two turning points can be defined clearly, near 20 and $50 \mathrm{~N} \cdot \mathrm{m}$, prior to the failure as shown in Figure C.4. Rod failure occurred at the middle of the gage section. The fracture surface of the cladding (Figure C.5) appeared to be very serrated. A large tooth-shaped fragment was attached on the compressive side of the rod, but it broke off during specimen handling. The final fracture of the rod was seemingly related to the fracture of a fuel pellet near a pellet to pellet interface.

\section{Observations:}

- The moment at fracture for Scal1 is lower than the maximum moment experienced by Demo1 as discussed above. So, there is a substantial mechanical property difference between the two rods, although they have the same thickness of oxide layer and the same level of $\mathrm{H}$ content.

- Lateral surfaces of the rod were covered with a large number of equally spaced circumferential cracks throughout the gage section on both the front (facing operator) and the back sides of the rod. Whether the cracks were linked around the whole periphery of the rod is uncertain.

- Extensive subsurface spalling can be seen over the back or tensile side of the rod. It is worthwhile noting that circumferential cracks and spalling also occurred on Demo1 and several other rods, as discussed below. 


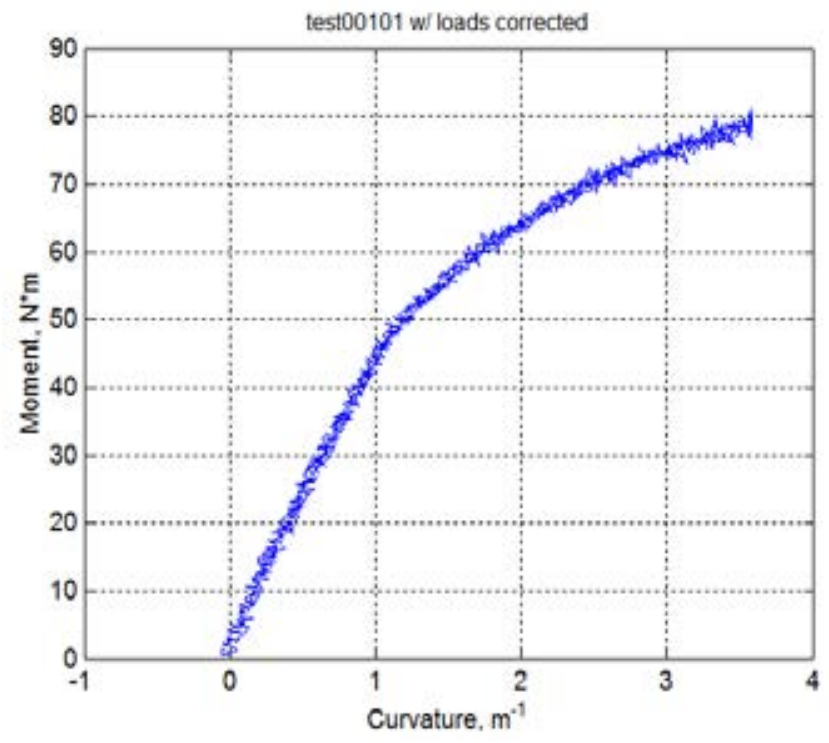

Figure C.4. Moment-curvature curve for Scal1/ Demo2 (606B3B) with a rate of 0.1 $\mathrm{mm} / \mathrm{sec}$ at the U-frame loading points. The rod broke at peak moment $=83 \mathrm{~N} \cdot \mathrm{m}$. 


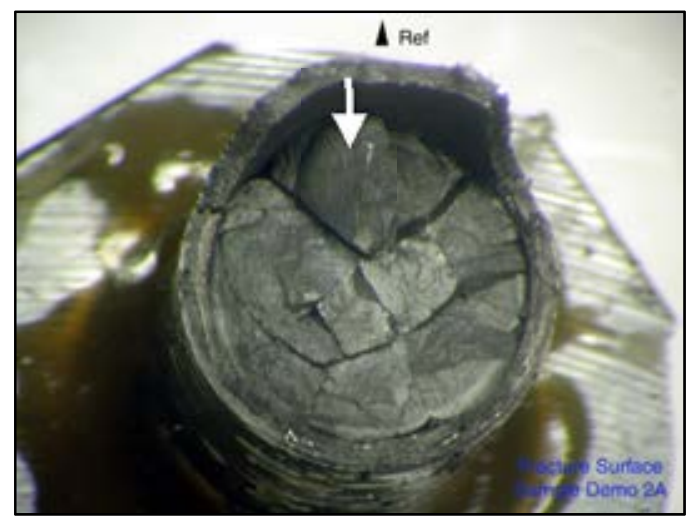

(a)

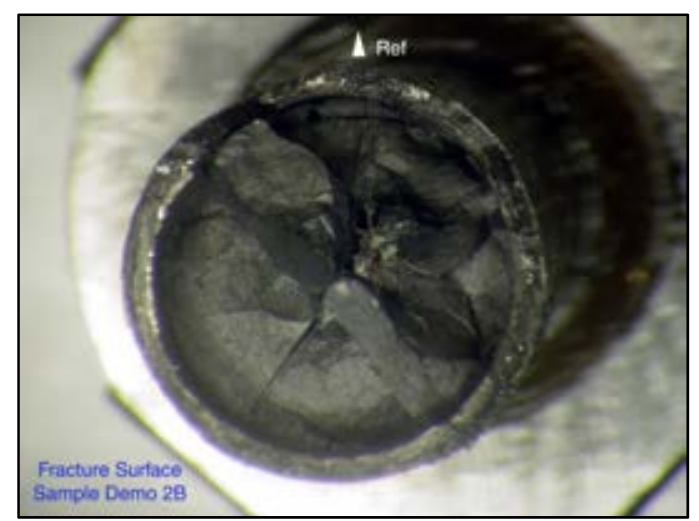

(b)

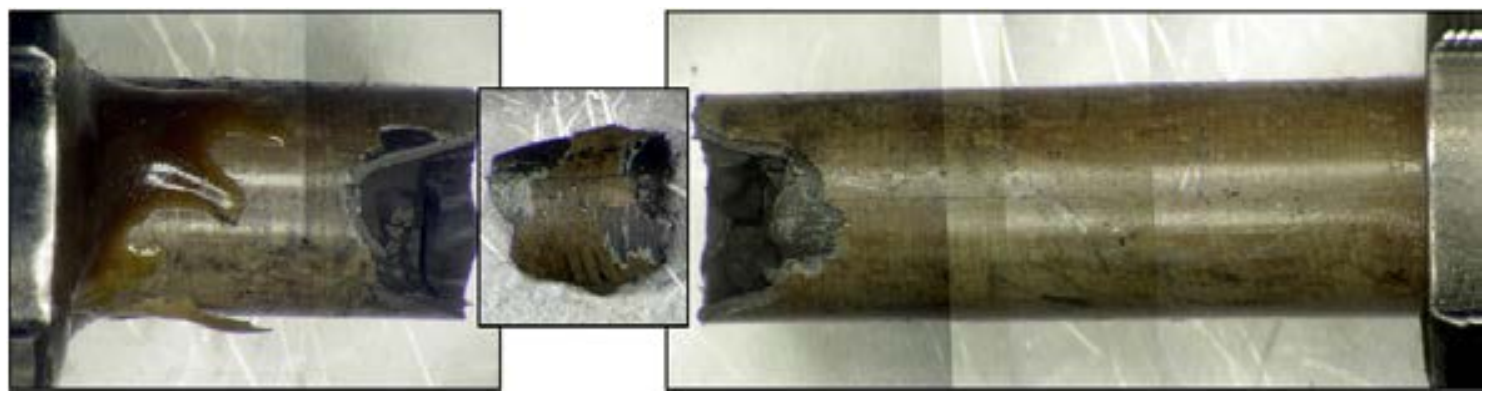

(c)
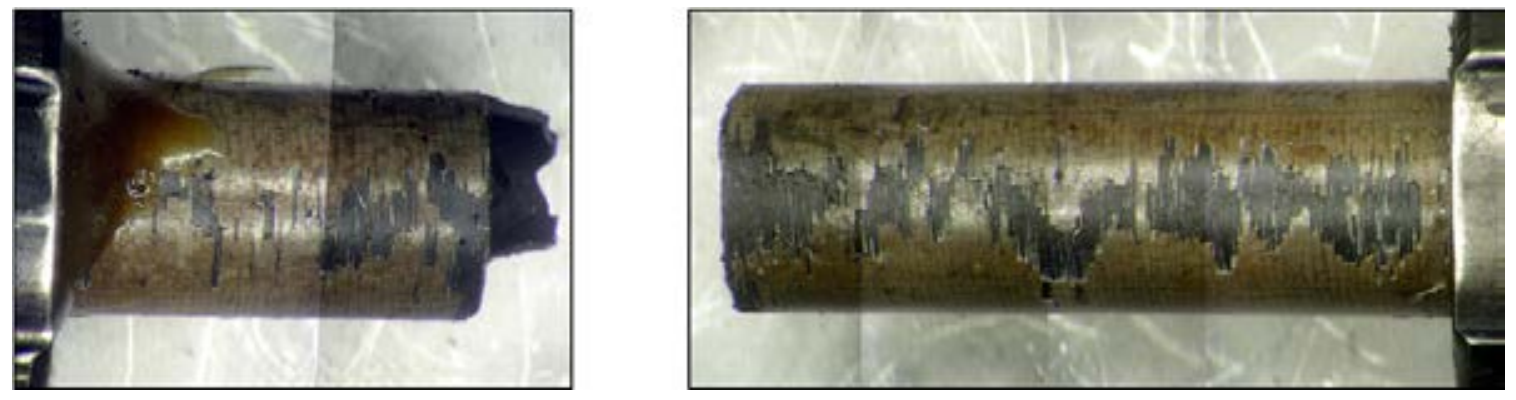

(d)

Figure C.5. (a, b) Mating fracture surfaces, (c) front side (compressive), and (d) back side (tensile) of Scal1/ Demo2 (606B3B). The rate was $0.1 \mathrm{~mm} / \mathrm{sec}$ at the U-frame loading points. The rod broke at peak moment $=78.2 \mathrm{~N} \cdot \mathrm{m}$.

\section{DYNAMIC TEST ON DCALI S3/ 605D1F ( $\pm 25.4 \mathrm{~N} \cdot \mathrm{M} 5 \mathrm{HZ}$ )}

Dynamic cycle testing was conducted on rod Dcal/ S3 (605D1F, 66.5 GWd/MTU burnup, 40-70 $\mu \mathrm{m}$ oxide layer, $360 \mathrm{ppm} \mathrm{H}$ content) as a part of system benchmarking. The cycle test was conducted under $\pm 250 \mathrm{~N}$ or $25.40 \mathrm{~N} \cdot \mathrm{m} 5 \mathrm{~Hz}$. The system has been demonstrated to meet the testing requirements for loading and monitoring. A lifetime of $2.5 \times 10^{4}$ cycles was observed. Flexural rigidity was shown to be fairly stable as the cycles accumulated prior to final fracture, as shown in Figure C.6. The associated measurements are shown in Figures C.7 and C.8. Failure was observed in the gage section near motor 2. A clear cut can be seen from the exposed end faces of neighboring pellets as shown in Figure C.9. 
- Rod failure was dominated by the pellet-to-pellet interface.

- The lateral surface of the rod was shown to be free of the cracks or spalling observed in Demo1, Scal1, and Scal2 (will be discussed below). It is not clear if the absence of circular cracks was related to the small loading amplitude or to the lesser degree of preexisting oxidation and hydrides in Dcal.

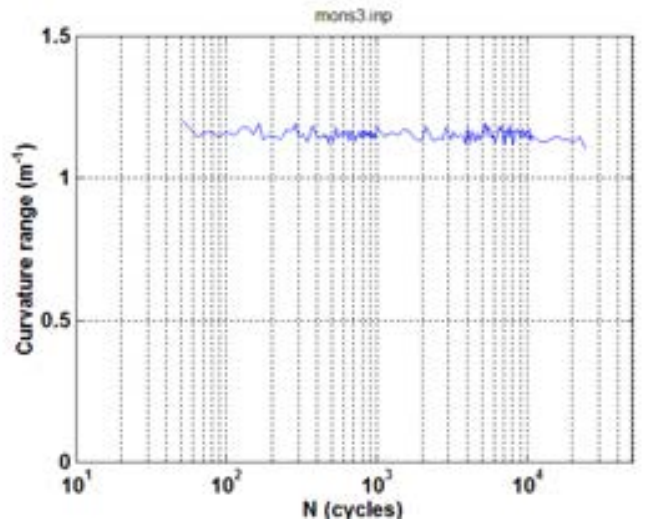

(a)

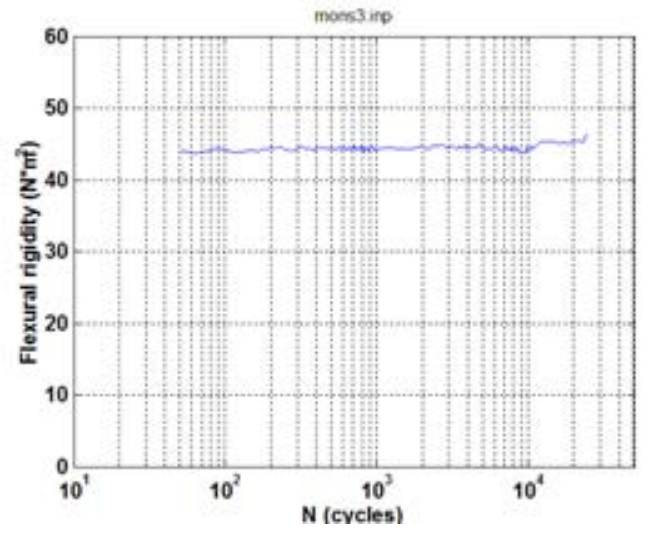

(c)

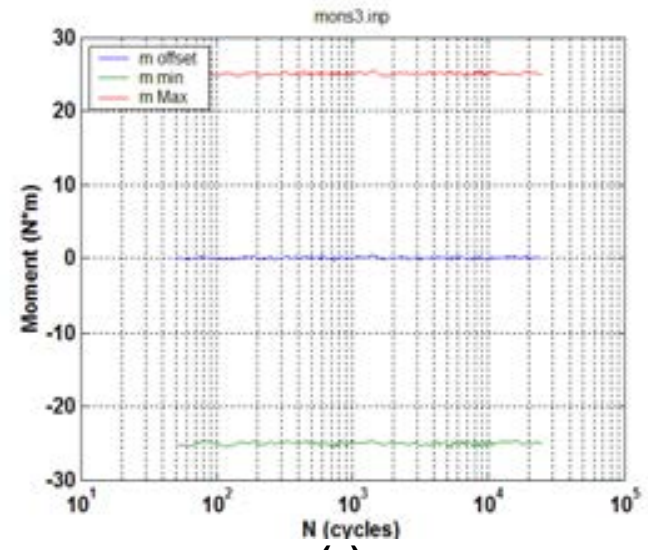

(e)

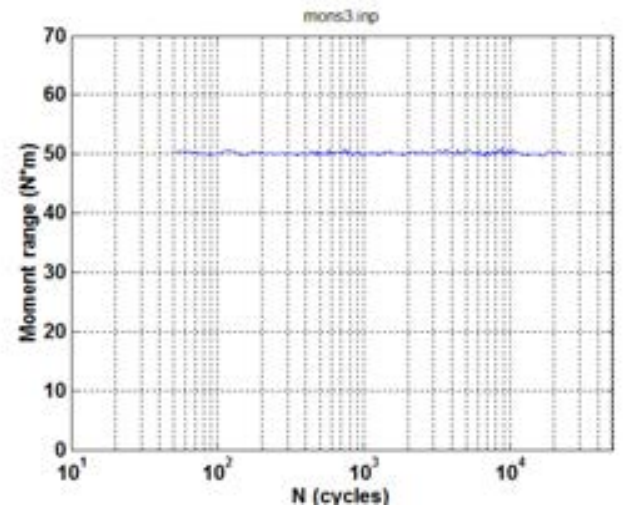

(b)

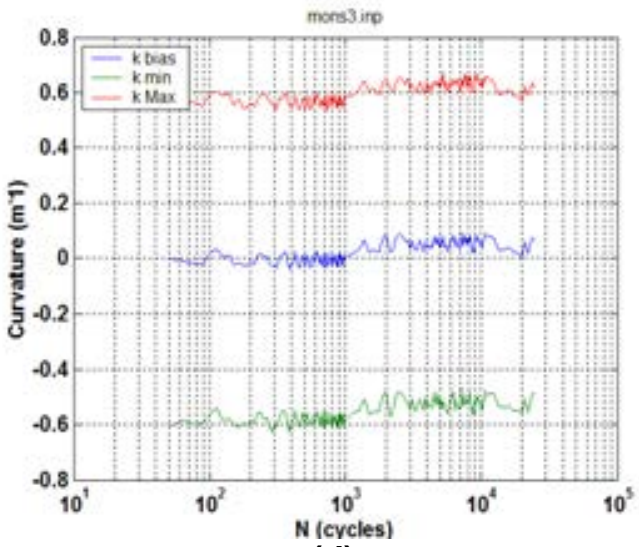

(d)

Figure C.6. Variations in (a) curvature range, (b) applied moment range, (c) flexural rigidity, (d) maximum and minimum values of curvature, and (e) maximum and minimum 
values of moment as a function of number of cycles for Dcal/ S3 (605D1F); $N_{f}=2.5 \times 10^{4}$ cycles under $\pm 25.4 \mathrm{~N} \cdot \mathrm{m} 5 \mathrm{~Hz}$.

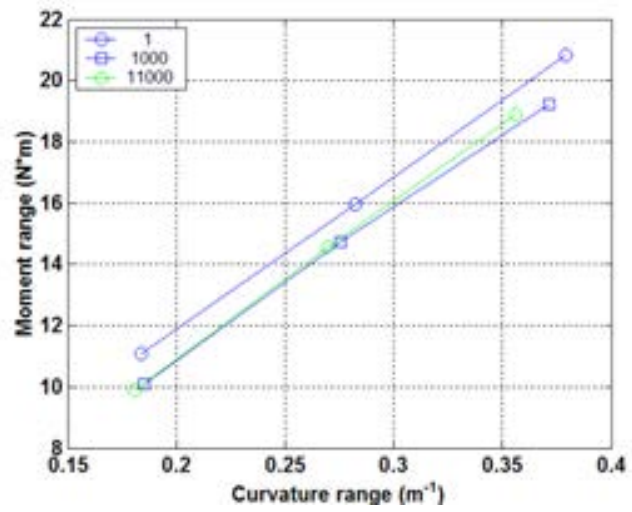

(a)

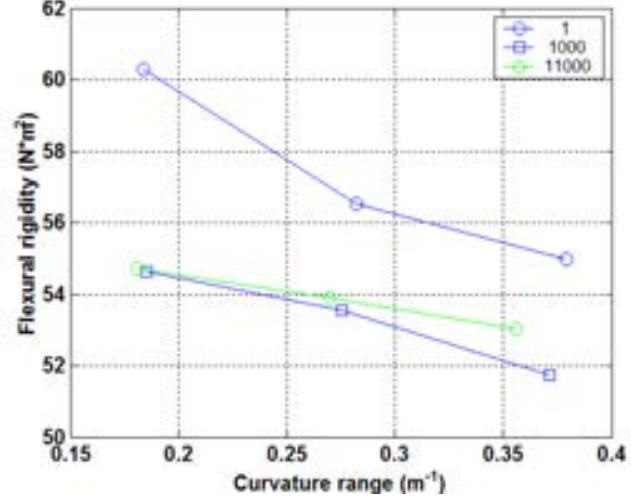

(b)

Figure C.7. (a) Moment-curvature relation, and (b) moment-flexural rigidity relation at various numbers of cycles for Dcal/ S3 (605D1F); $\mathrm{N}_{\mathrm{f}}=2.5 \times 10^{4}$ cycles under $\pm 25.4 \mathrm{~N} \cdot \mathrm{m}$ $5 \mathrm{~Hz}$.

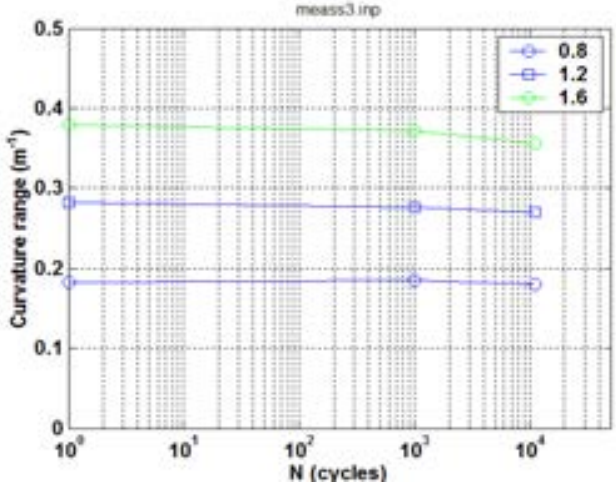

(a)

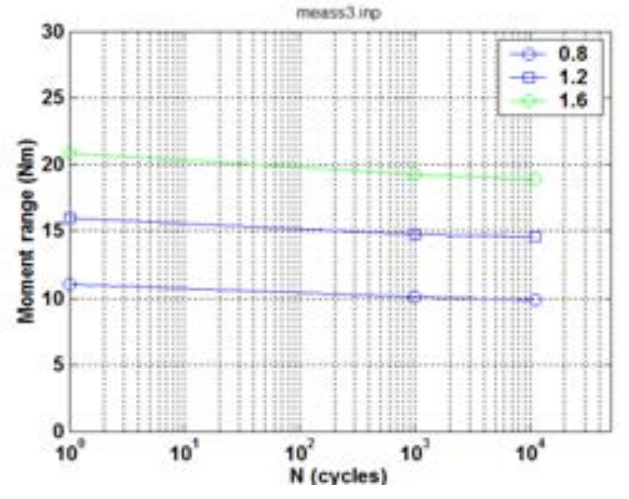

(b)

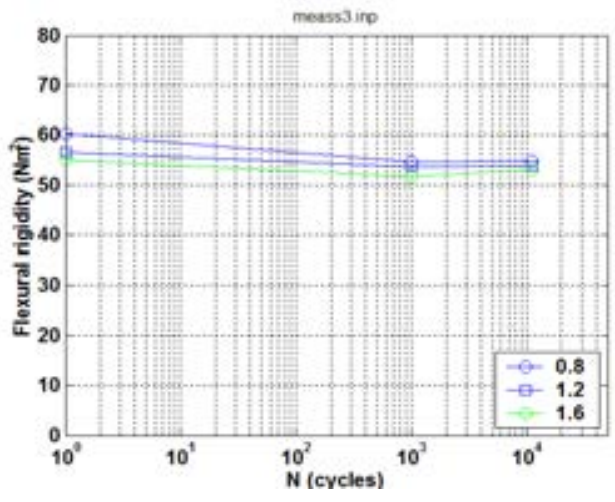

(c)

Figure C.8. Variations in (a) curvature range, (b) applied moment range, and (c) flexural rigidity as a function of number of cycles for Dcall S3 (605D1F); $N_{f}=2.5 \times 10^{4}$ cycles under $\pm 25.4 \mathrm{~N} \cdot \mathrm{m} 5 \mathrm{~Hz}$. 


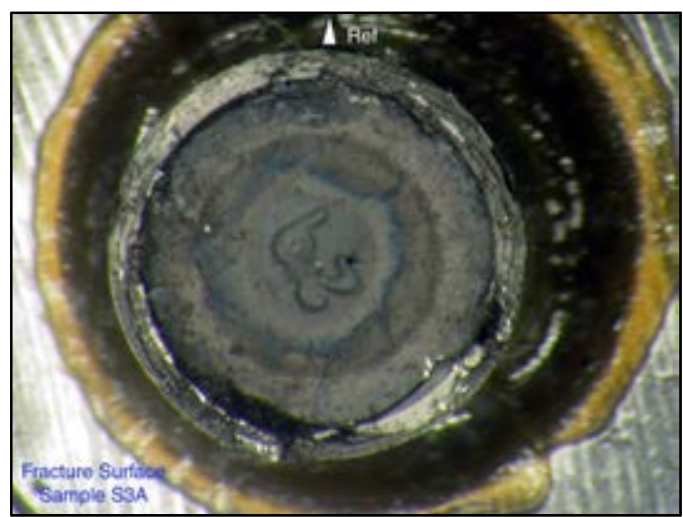

(a)

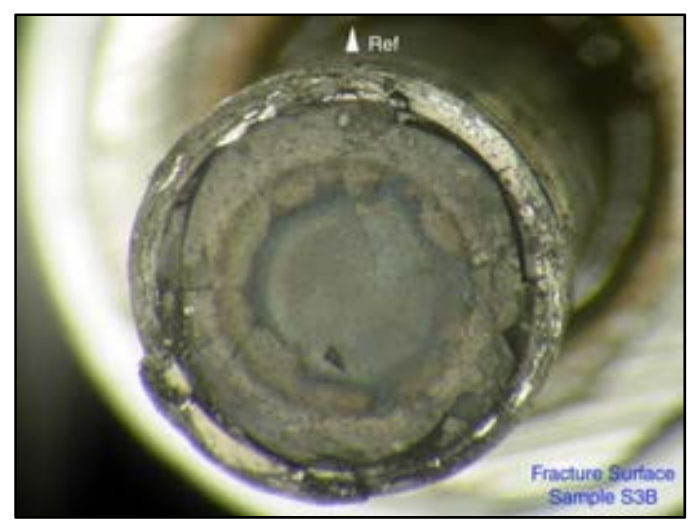

(b)
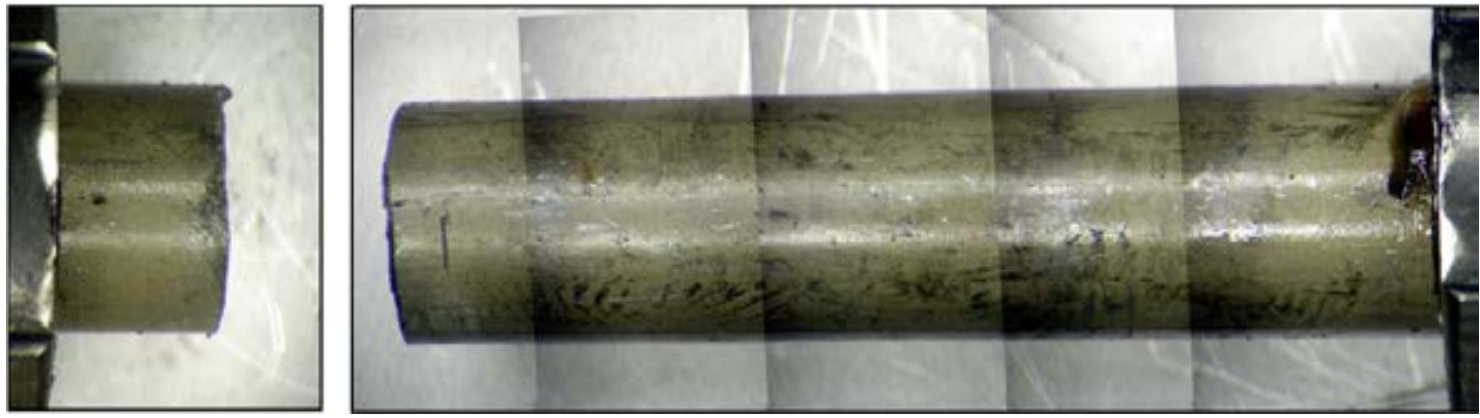

(c)
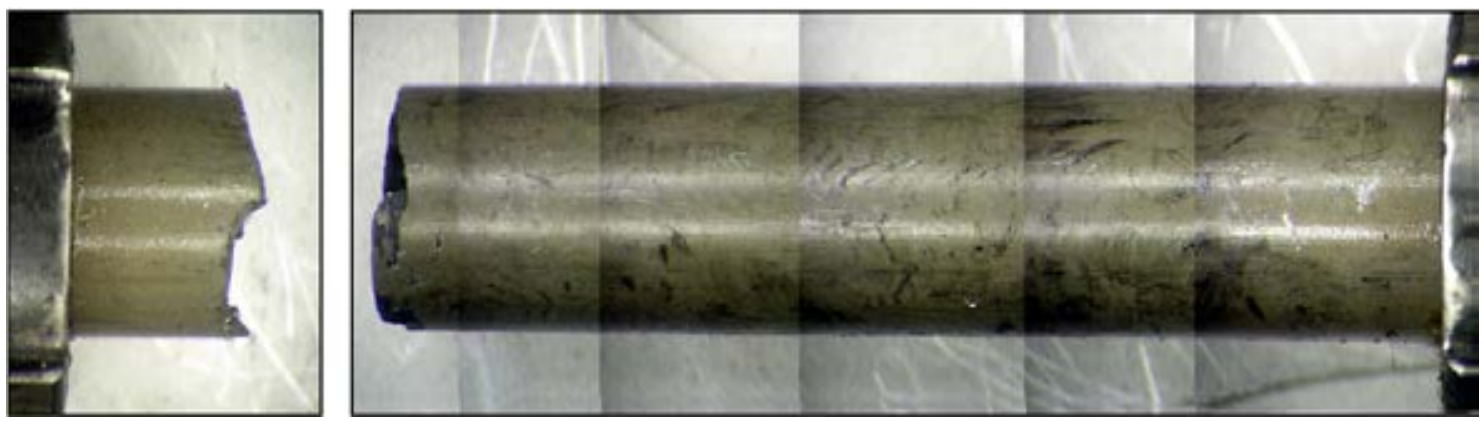

(d)

Figure C.9. (a, b) Mating fracture surfaces, (c) front side, and (d) back side of Dcal/ S3 (605D1F); $\mathrm{N}_{\mathrm{f}}=2.5 \times 10^{4}$ cycles under $\pm 25.40 \mathrm{~N} \cdot \mathrm{m} 5 \mathrm{~Hz}$.

\section{STATIC TEST ON SCAL2/ S4/ 606C3B}

Scal2/ S4 (606C3B, 66.8 GWd/MTU burnup, 70-100 $\mu \mathrm{m}$ oxide layer, 700 ppm H content) fractured at the peak moment near $80.7 \mathrm{~N} \cdot \mathrm{m}$ at a loading of $0.1 \mathrm{~mm} / \mathrm{s}$. A large variety of fragment sizes were revealed in the postmortem analysis (Figure C.10).

- The end face of a pellet can be seen clearly from the brownish haze zone. So, the failure took place near a pellet-to-pellet interface with the fracture involving a neighboring pellet. 
- Besides the circumferential cracks, a sharp attachment of cladding was outstanding on the frontal or compressive side. On the other side, spalling was not as extensive as in Demo1 and Scal1.

- Compared to Scal1 from the same fuel rod but a different segment (606B), the slightly higher failure moment and less spalling in Scal2 are coincident with the lower levels of oxide-layer thickness and $\mathrm{H}$ content of the rod segment.

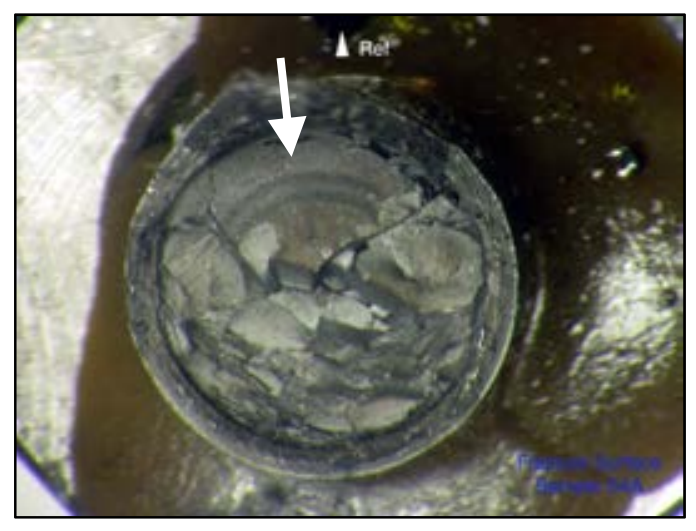

(a)
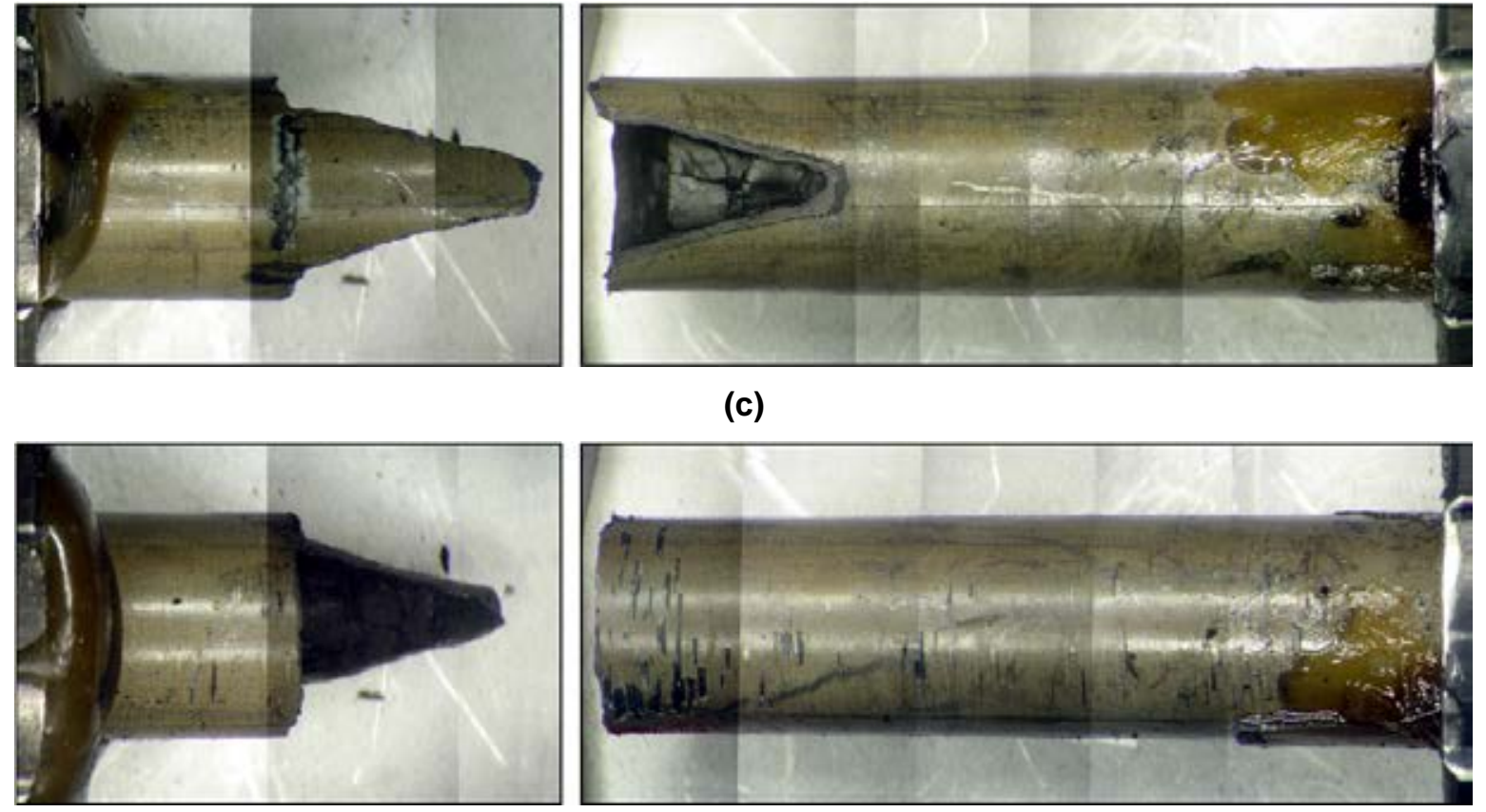

(d)

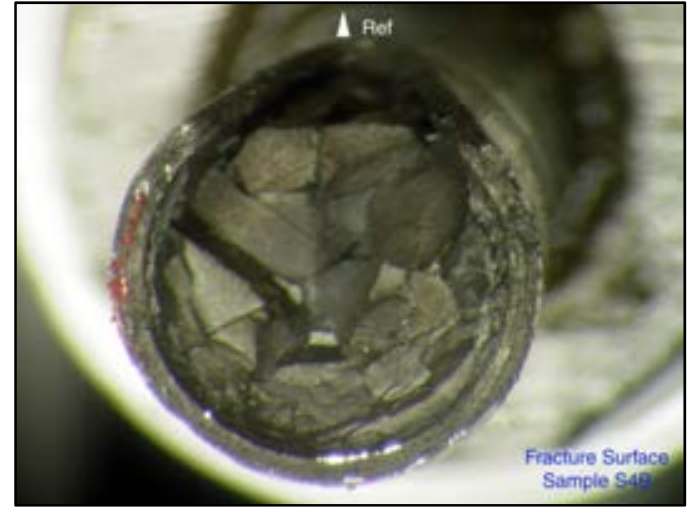

(b)

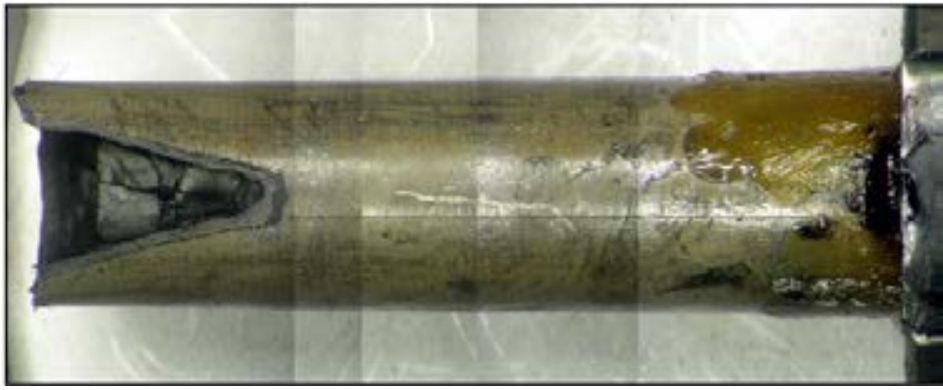

(c) Figure C.10. (a, b) Mating fracture surfaces, (c) front (compressive) side, and (d) back
(tensile) side of Scal2/ S4 (606C3B). The rate at the U-frame loading points was $0.1 \mathrm{~mm} / \mathrm{sec}$ The rod broke at peak moment $=80.7 \mathrm{~N} \cdot \mathrm{m}$. 

APPENDIX D

STATIC TEST RESULTS 

Static tests were conducted on October 7-8, 2013. A brief description of four static tests and their results is given below.

\section{S1/ 606C3C (+ DYNAMIC)}

S1 (606C3C, $66.8 \mathrm{GWd} / \mathrm{MTU}$ burnup, 70-100 $\mu \mathrm{m}$ oxide layer, $650 \mathrm{ppm} \mathrm{H}$ content) was tested under four repeated static loading cycles before being taken into a dynamic cyclic test. The moment-curvature curve and equivalent stress-strain curve for the initial cycle are presented in Figure D.1 (the curves of subsequent cycles overlaid to a great extent and are not plotted for clarity). Again, the turning points were revealed near 12 and $59 \mathrm{~N} \cdot \mathrm{m}$. The rod fractured in a follow-up cyclic test under \pm 25.40 to $30.48 \mathrm{~N} \cdot \mathrm{m} 5 \mathrm{~Hz}$ near $1.4 \times 10^{4}$ cycles.

Fracture occurred near motor 1 (right side) with a fuel release of 0.9 grams. Fractography using an optical microscope as shown in Figure D.2 revealed that the rod failure was related to fractures of a pellet adjacent to a pellet-to-pellet interface. The exit of the pellet end-face can be identified from the haze zone characterized by the brownish blue color. At the same time, a serrated fracture surface of cladding was observed with a slender attachment (about one roddiameter length).

The lateral surfaces [Figure D.2 (c, d)] near the rod's neutral axis of bending were free of circumferential cracks to a great extent. On the other hand, one of the stressed lateral surfaces was covered with a large number of circumferential cracks and related subsurface spalling [Figure D.2 (d)]. It is uncertain if the spalling was related to tensile stress in static loading as the specimen ID was $90^{\circ}$ off to the stressed sides.

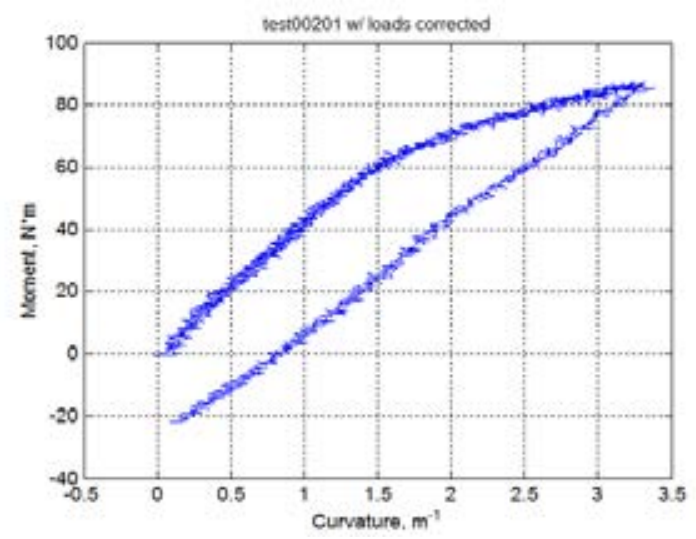

(a)

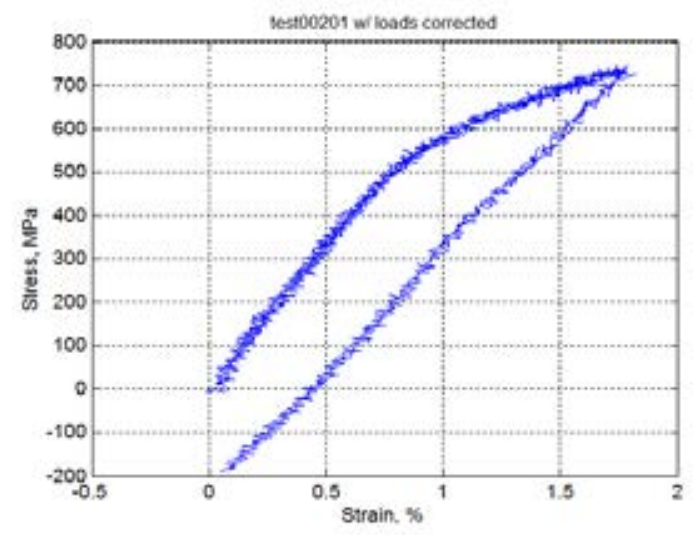

(b)

Figure D.1. (a) Curve of moment versus curvature, and (b) equivalent strain-stress curve for $\mathrm{S1}(606 \mathrm{C} 3 \mathrm{C})$, based on static loading cycle in which the maximum relative displacement was $24 \mathrm{~mm}$ and the rate at the U-frame loading points was set at 0.1 and 0.2 $\mathrm{mm} / \mathrm{s}$ in loading and unloading, respectively. 


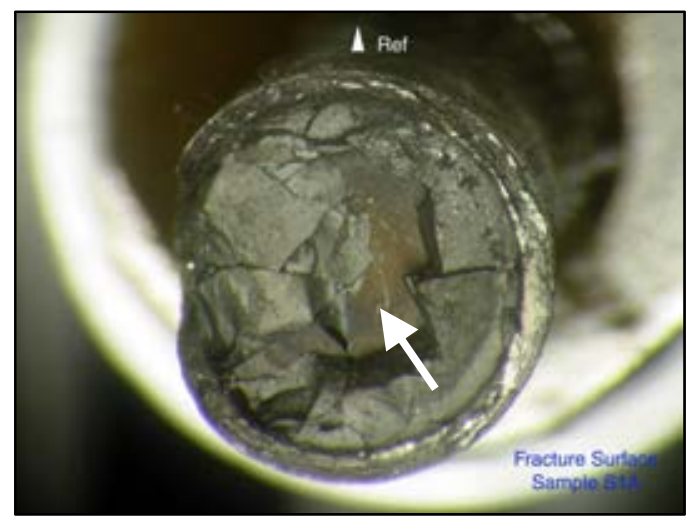

(a)

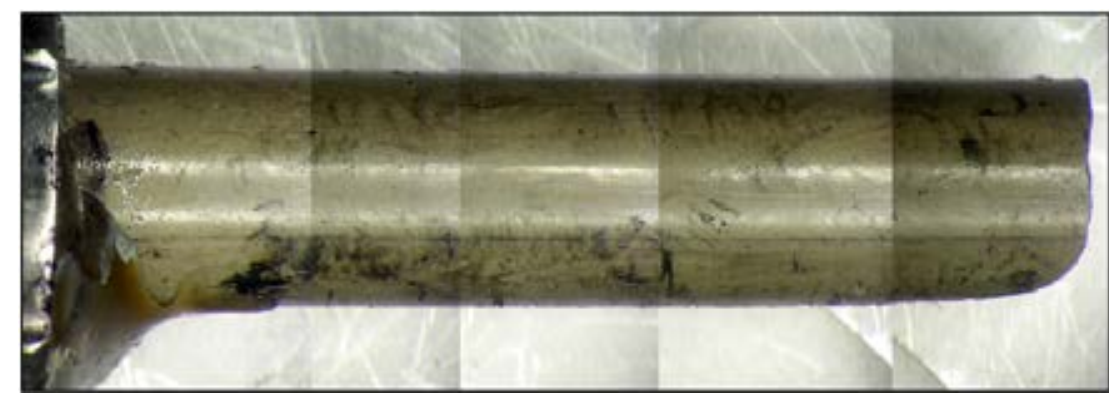

(c)

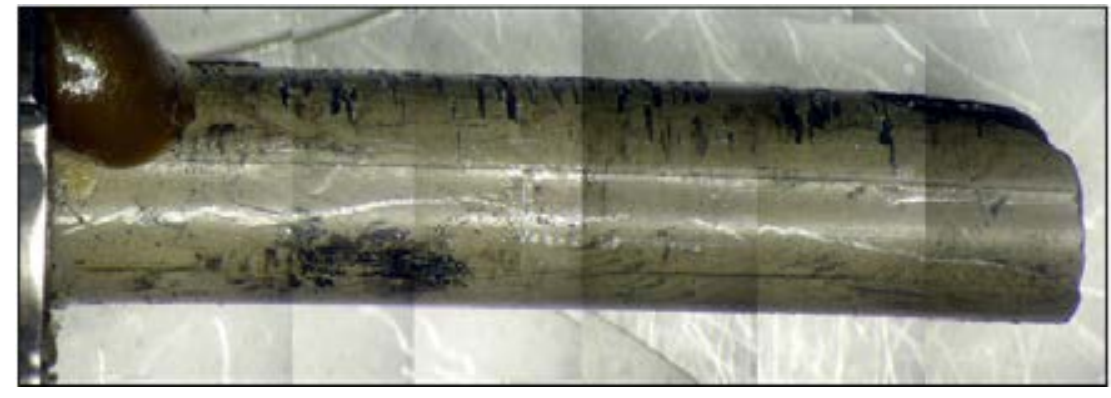

(d)
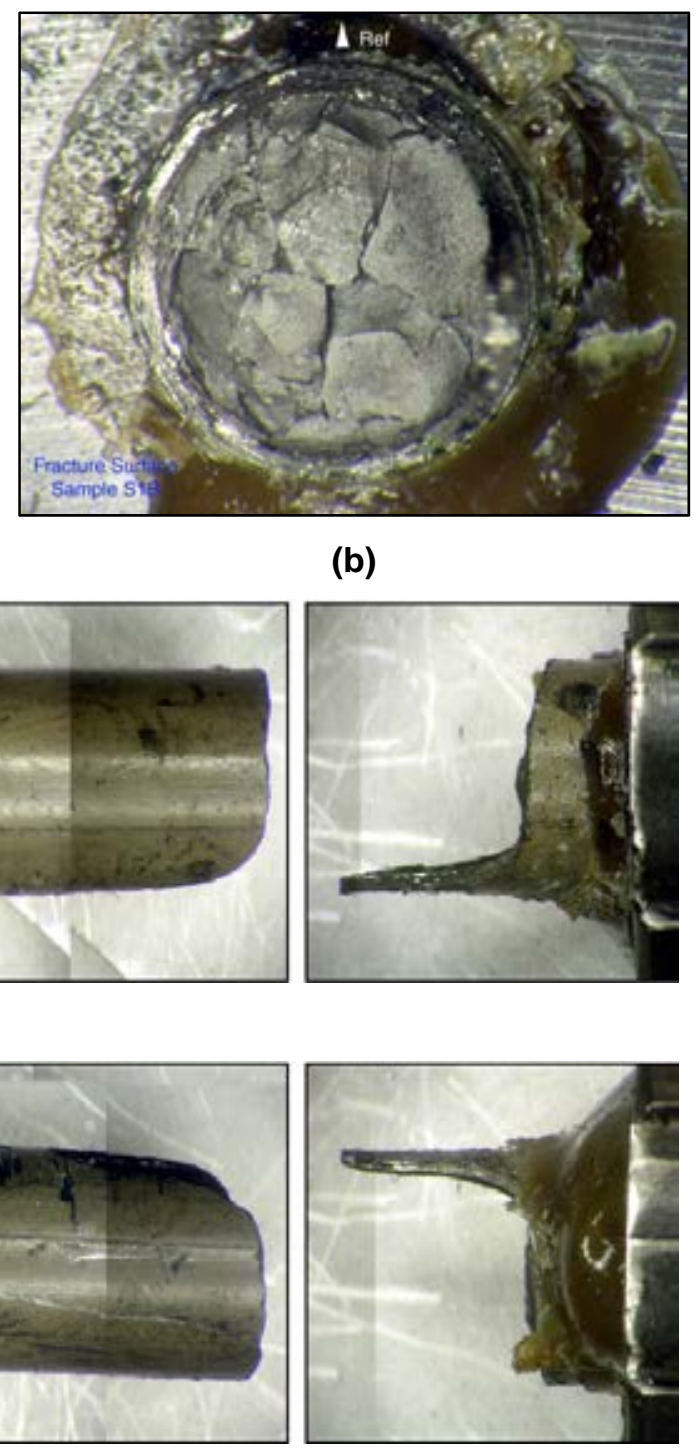

(b)

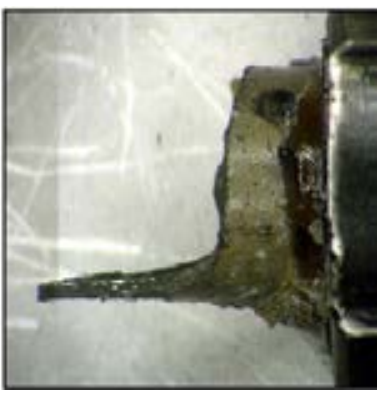

Figure D.2. (a, b) Mating fracture surfaces and $(c, d)$ lateral sides near the rod's neutral axis of bending for $\mathrm{S} 1$ (606C3C), which was subjected to four repeated static cycles to $24 \mathrm{~mm}$ maximum relative displacement followed by dynamic cyclic loading $\mathbf{2 5 . 4 0}$ to $30.48 \mathrm{~N} \cdot \mathrm{m} 5 \mathrm{~Hz} ; 1.4 \times 10^{4}$ failure cycles, $0.9 \mathrm{gram}$ of fuel particles collected.

\section{S2/ 605D1E (+ DYNAMIC)}

S2 (605D1E, $66.5 \mathrm{GWd}$ /MTU burnup, 40-70 $\mu \mathrm{m}$ oxide layer, 400 ppm H content) was tested by following a routine similar to that of $\mathrm{S} 1$. The specimen survived three cycles of large displacement loading and $7.2 \times 10^{3}$ cycles under $\pm 30.48 \mathrm{~N} \cdot \mathrm{m} 5 \mathrm{~Hz}$ before failure at the middle of the gage section.

The moment-curvature curve and equivalent stress-strain curve for the initial cycle are presented in Figure D.3. Again, the turning points were revealed near 20 and $62 \mathrm{~N} \cdot \mathrm{m}$. 
Fracture occurred on a pellet-to-pellet interface as can be seen from Figure D.4, in which the smooth brownish end face of the adjacent pellet can be seen clearly. One of the attachments [Figure D.4 (b)] is convex-shaped and covers almost $1 / 3$ of the end face, and several small ones appear around the peripheral area.

The cladding fracture appeared very serrated with a sizable tooth-shaped attachment on the compressive side of the rod. The lateral stressed surface of the rod was covered by sparsely distributed circumferential spalling. The rod's minor damaged lateral surface of bending agrees with the results of Dcal/ S3 as they were prepared from same segment (605D) with the same level of preexisting oxides and hydrides.

Fuel release was about 0.6 grams.

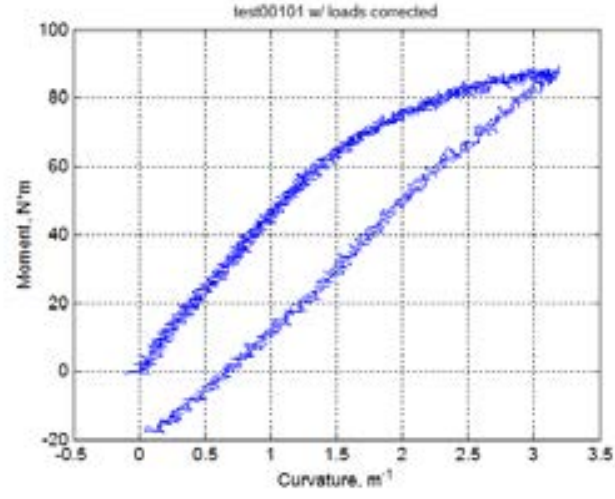

(a)

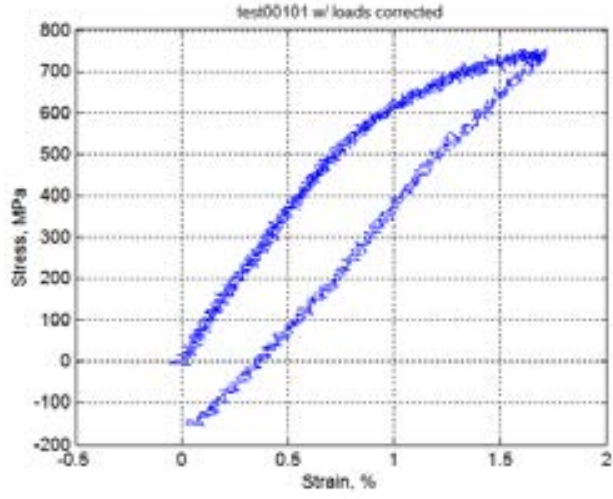

(b)

Figure D.3. (a) Curve of moment versus curvature and (b) equivalent strain-stress curve for S2 (605D1E), based on static loading cycle in which maximum relative displacement was $24 \mathrm{~mm}$ and the rate at the U-frame loading points was set at 0.1 and $0.2 \mathrm{~mm} / \mathrm{s}$ in loading and unloading, respectively. 


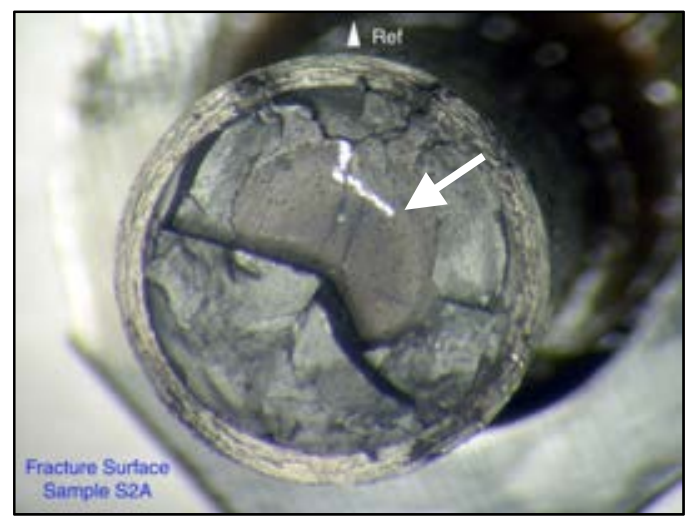

(a)

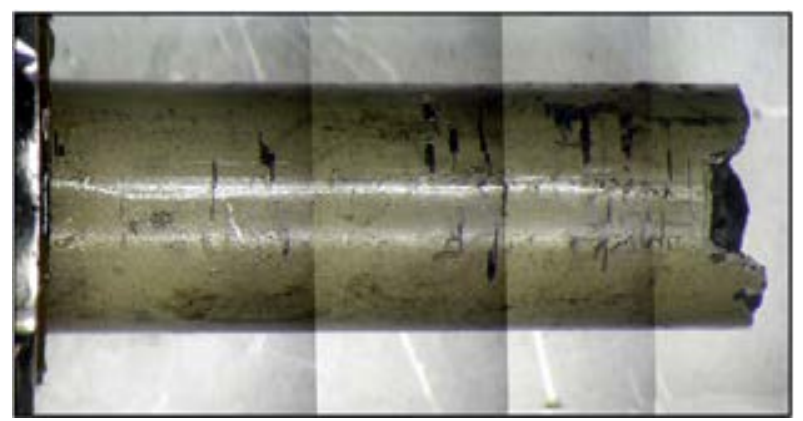

(c)

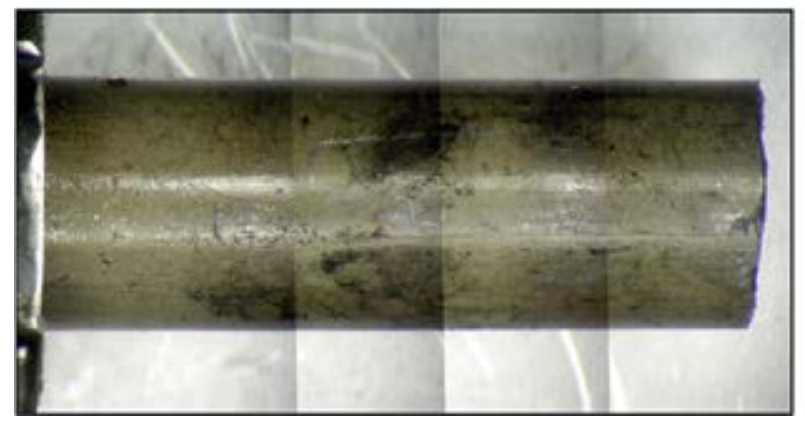

(d)

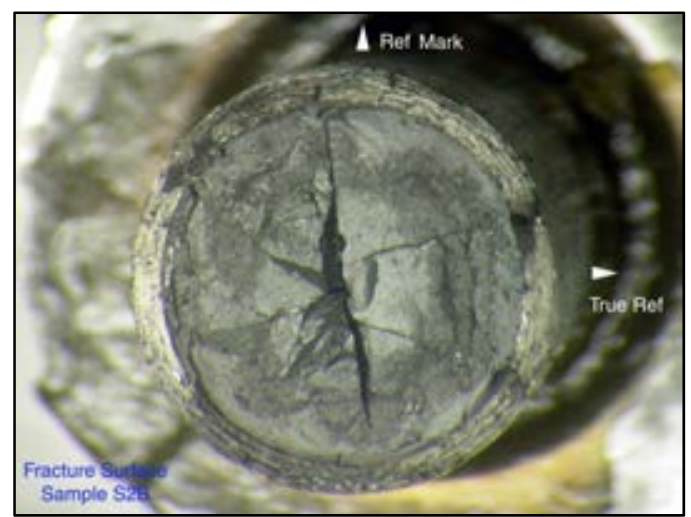

(b)
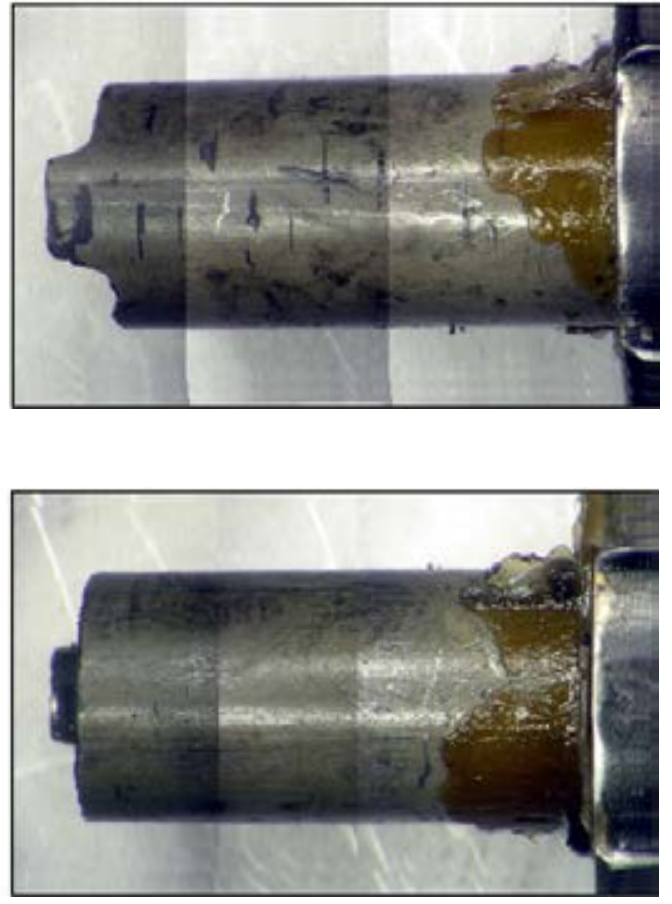

Figure D.4. (a, b) Mating fracture surfaces, (c) front side, and (d) back side of S2 (605D1E), which was subjected to three repeated static cycles to $24 \mathrm{~mm}$ maximum relative displacement followed by dynamic cyclic loading $\pm 30.48 \mathrm{~N} \cdot \mathrm{m} 5 \mathrm{~Hz} ; 7.2 \times 10^{3}$ failure cycles, $\mathbf{0 . 6}$ gram of fuel particles collected.

\section{S3I DCALI 609C5 (+ DYNAMIC)}

S3/Dcal (609C5, 66.5 GWd/MTU burnup, 70-100 $\mu \mathrm{m}$ oxide layer, 550 ppm H content) was subjected to three repeated static cycles of $24 \mathrm{~mm}$ displacement loading followed by cyclic loading $\pm 30.48 \mathrm{~N} \cdot \mathrm{m} 5 \mathrm{~Hz}$. The S3 failed around $9.6 \times 10^{3}$ cycles at the middle of the gage section with about 0.2 gram of fuel released. 
A three stage moment-curvature curve was obtained as shown in Figure D.5, in which two turning points can be seen near 18 and $60 \mathrm{~N} \cdot \mathrm{m}$.

Rod failure was seemingly related to the fracture of a pellet (Figure D.6). No involvement of the pellet-to-pellet interface can be seen. The fracture surface of the pellet was characterized by attached fragments whose size was about a quarter the diameter of a pellet.

A serrated fracture surface was seen again over the cladding. Furthermore, the fracture surface was tilted to a certain degree to the longitudinal axis of the rod. This is in contrast to those rods whose failure actively involves the pellet-to-pellet interface, where the fracture surfaces are more or less normal to the longitudinal axis of the rod. Circumferential cracks and spalling can be observed on lateral stressed sides, especially on the tensile side in static loading. The spalls had an extended dimension in the circumferential direction, some of which were overlaid in the longitudinal axis of the rod. However, the extent of overlaying is not as large as seen in Demo1 and Scal1/ Demo2, attributed to less development of oxides and hydrides.

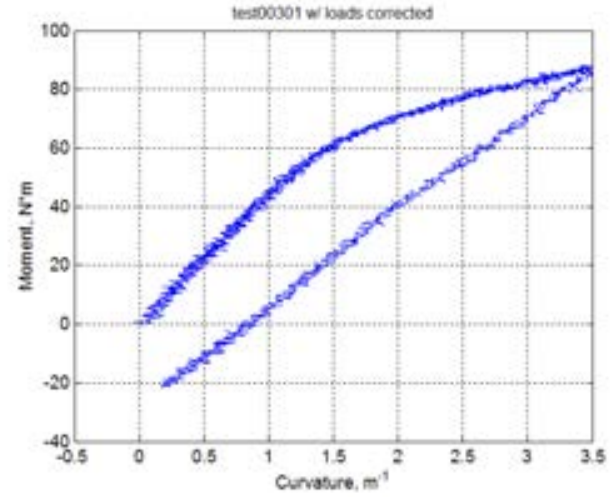

(a)

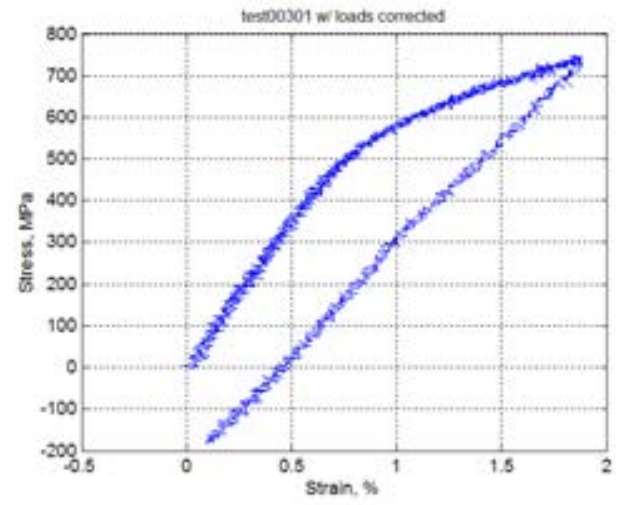

(b)

Figure D.5. (a) Curve of moment versus curvature and (b) equivalent strain-stress curve for S3/ Dcal (609C5), based on a static loading cycle in which maximum relative displacement was $24 \mathrm{~mm}$ and the rate at the U-frame loading points was set at 0.1 and 0.2 $\mathrm{mm} / \mathrm{s}$ in loading and unloading, respectively. 


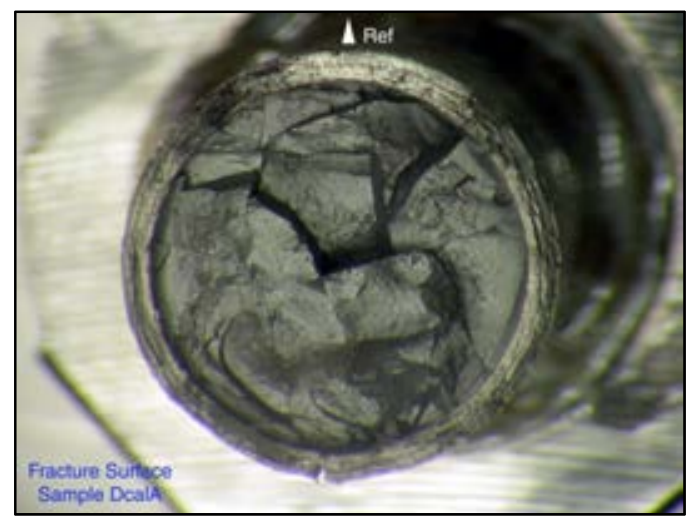

(a)

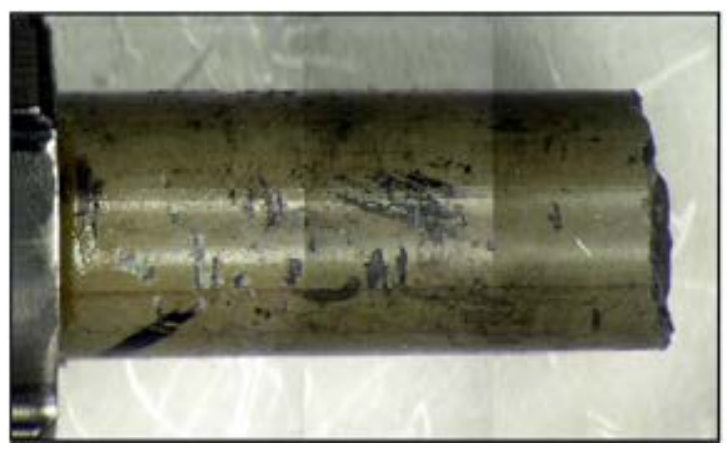

(c)

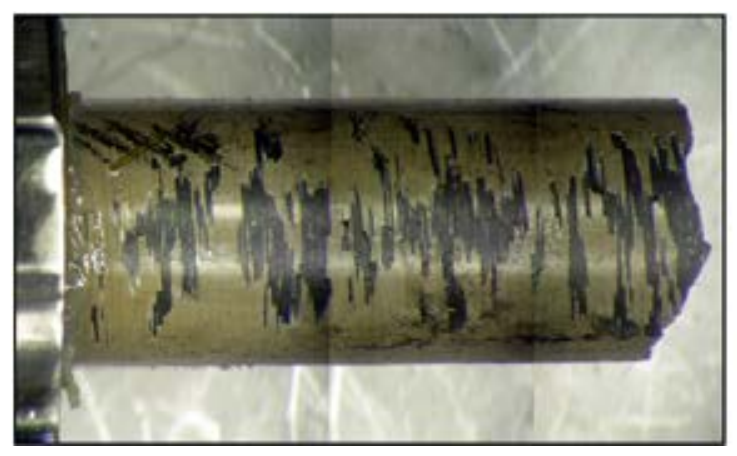

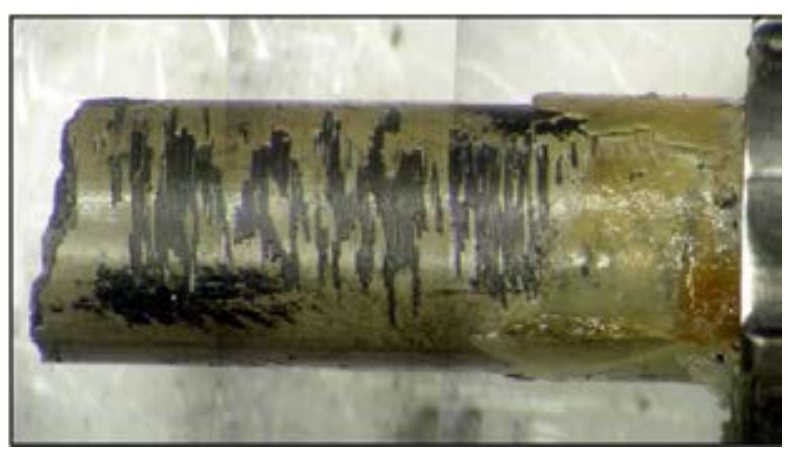

(d)

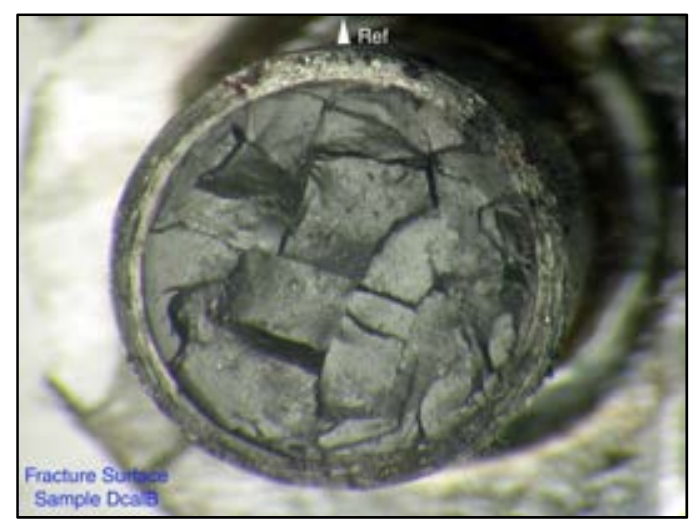

(b)

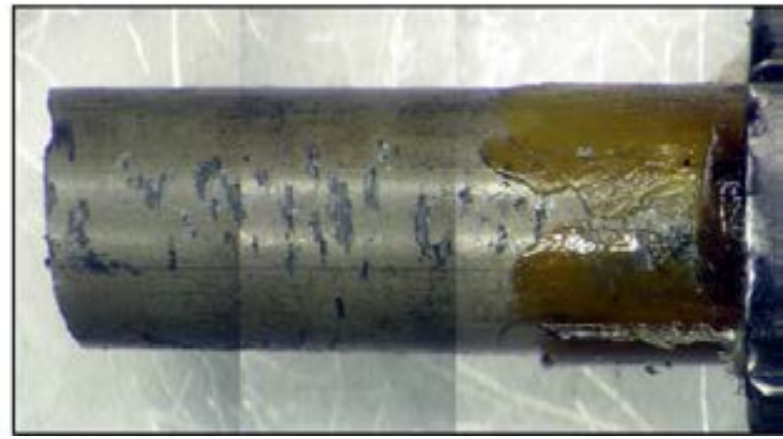

\section{(c)}

Figure D.6. (a, b) Mating fracture surfaces, (c) front side, and (d) back side of S3/ Dcal (609C5), which was subjected to three repeated static loadings to $24 \mathrm{~mm}$ maximum relative displacement followed by dynamic loading: $\pm 30.48 \mathrm{~N} \cdot \mathrm{m} 5 \mathrm{~Hz} ; 9.6 \times 10^{3}$ failure cycles, 0.2 gram of fuel particles collected.

\section{S4/ SCAL/ $609 C 6$}

S4/ Scal (609C6, 66.5 GWd/MTU burnup, 70-100 $\mu \mathrm{m}$ oxide layer, 550 ppm H content) sustained three cycles of loading with relative displacement of $24 \mathrm{~mm}$ on the U-frame and an additional load with $25 \mathrm{~mm}$ before failure. Failure occurred around the peak moment $86.1 \mathrm{~N} \cdot \mathrm{m}$. The curves for the initial cycle exhibited a smooth transit from linear to nonlinear as shown in 
Figure D.7. However, the turning point near $20 \mathrm{~N} \cdot \mathrm{m}$ is still appreciable based on the discontinuous change in the slope of the moment-curvature curve.

Fracture occurred near motor 2 (left side) and was shown to be related to pellet fractures near a pellet-to-pellet interface as illustrated in Figure D.8. The exit of the end face of the involved pellet can be seen clearly from the bluish haze zone. A serrated fracture surface of cladding can also be seen.

The specimen ID was found to be $90^{\circ}$ off the stressed surfaces of the rod. A conspicuous sharp attachment on one side suggests where the intensified compression once resided, inducing the shearing fracture. The opposite side is covered by a certain amount of spalling as discussed above. The less stressed lateral side near the rod's neutral axis of bending appeared to be clear of circumferential cracks.

Failure of the rod involved a lot of fragmentation in the fractured pellet. The released fuel weighed 1.3 grams.

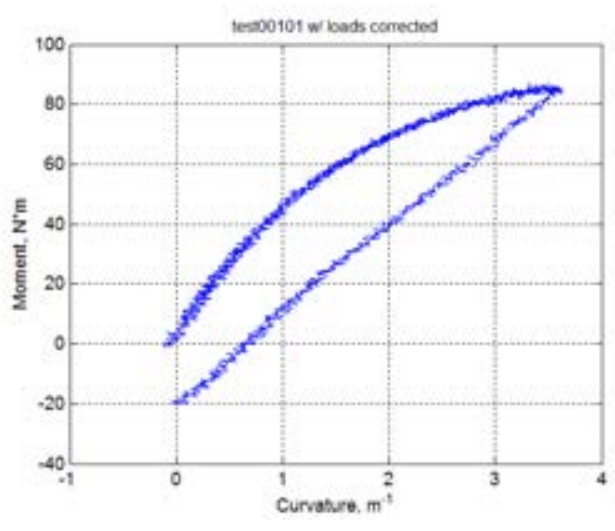

(a)

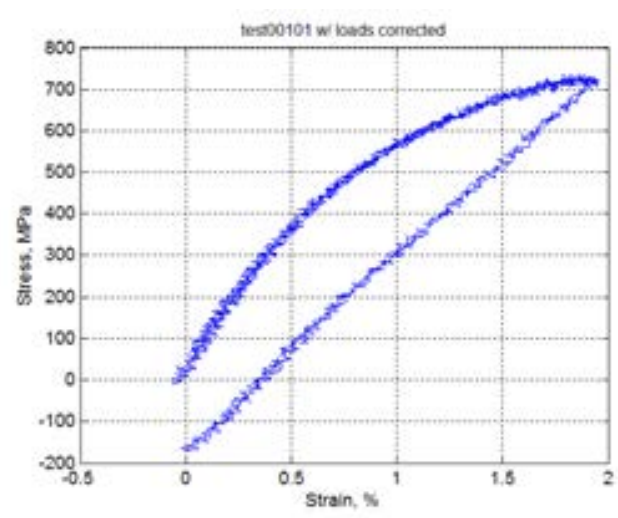

(b)

Figure D.7. (a) Curve of moment versus curvature and (b) equivalent strain-stress curve for S4/ Scal (609C6), based on static loading cycle in which maximum relative displacement was $24 \mathrm{~mm}$ and the rate at the U-frame loading points was set at 0.1 and 0.2 $\mathrm{mm} / \mathrm{s}$ in loading and unloading, respectively. 


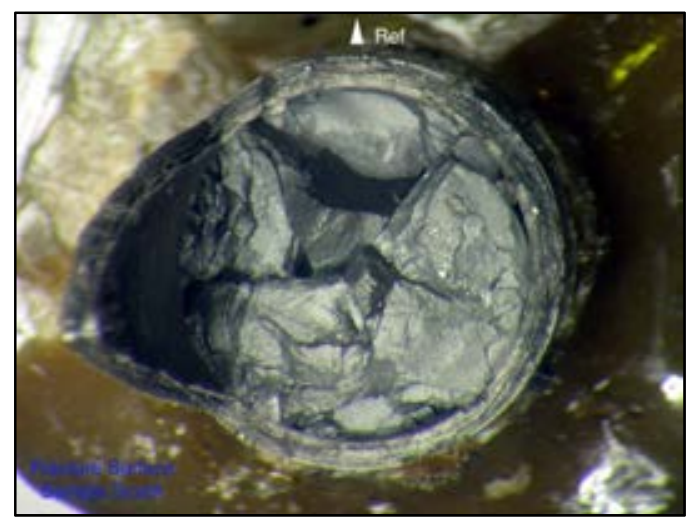

(a)
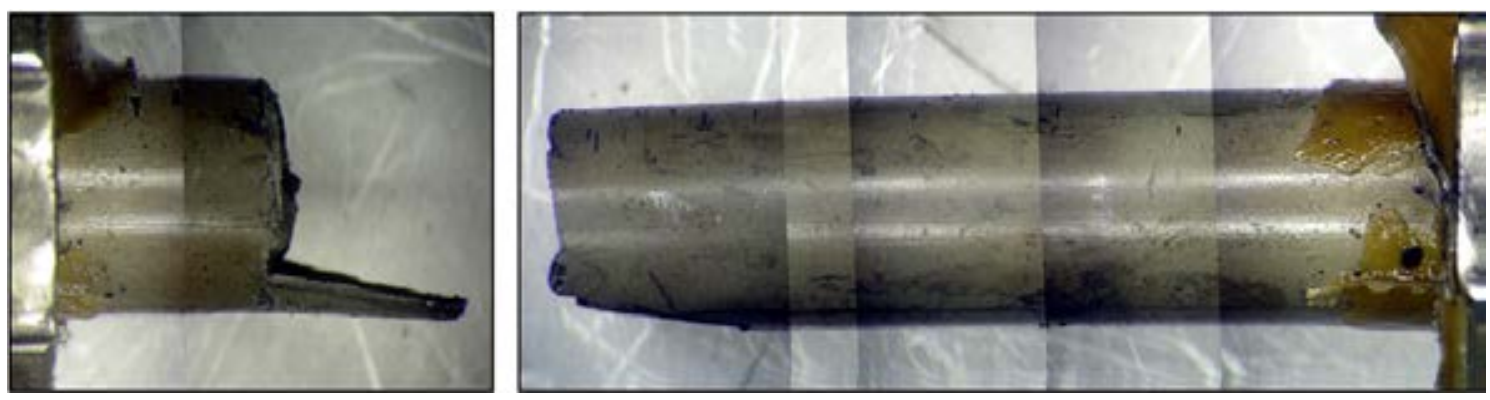

(c)
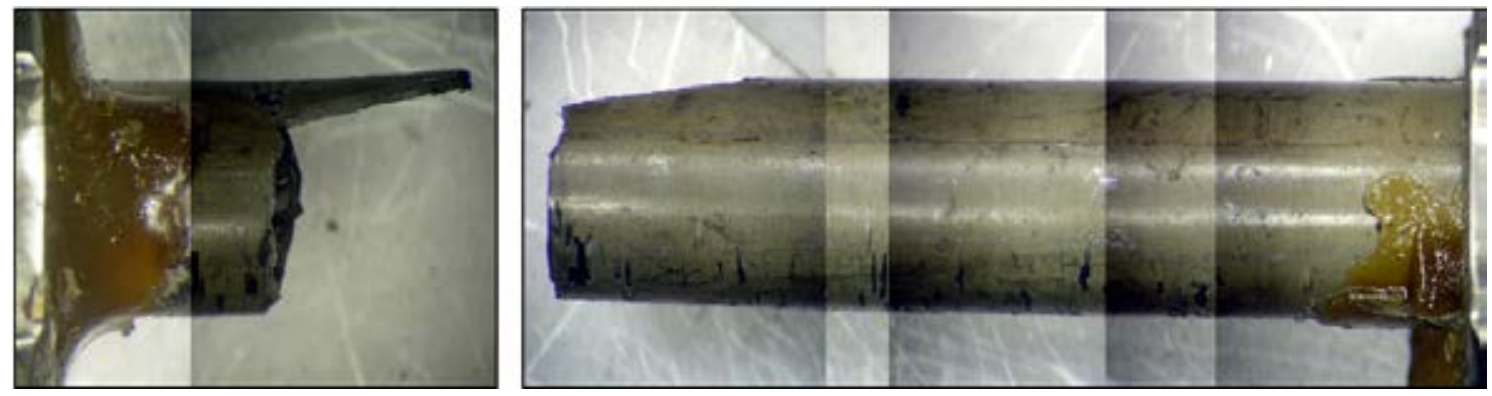

(d)

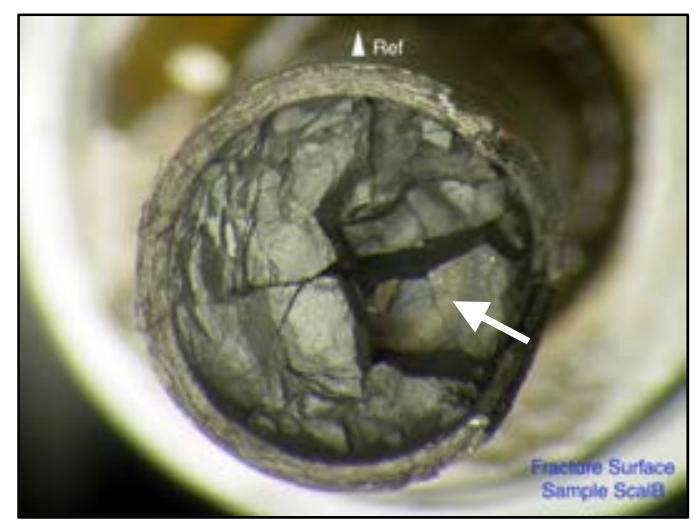

(b) 


\section{APPENDIX E}

\section{DYNAMIC TEST RESULTS}



Dynamic testing began on October 22, 2013, and is still ongoing. A description of completed dynamic tests and their results is given below.

\section{D1/ DL1/ 607C4B ( $\pm 15.24 \mathrm{~N} \cdot \mathrm{M} 5 \mathrm{HZ})$}

The test on D1/ DL1 (607C4B, $63.8 \mathrm{GWd/MTU}$ burnup, 70-100 $\mu \mathrm{m}$ oxide layer, 700 ppm H content) was conducted under $\pm 15.24 \mathrm{~N} \cdot \mathrm{m} 5 \mathrm{~Hz}$. A lifetime of $1.1 \times 10^{5}$ cycles was obtained with less than 1 gram of fuel particles collected. The cycle to failure of DL1 is apparently longer than that obtained for Dcal. The latter was subjected to a higher amplitude load, $25.40 \mathrm{~N} \cdot \mathrm{m}$.

Measurements were conducted under displacement control at two relative displacement levels: 0.8 and $1.2 \mathrm{~mm}$. The curvature-moment loops obtained for $\mathrm{N}=1$ and 111,000 cycles are given in Figure E.1. It is interesting that the loops actually became quite slender with the accumulated cycles, whilst the amplitude of the loops indeed decreased to a certain degree. The ranges of moment in relation to curvature and flexural rigidity are illustrated in Figure E.2. It can be seen that most of the rigidity degradations occurred in the first 1,000 cycles. Variations of these quantities as a function of number of cycles are shown in Figure E.3. While the curvature stayed at a quite consist level as a result of displacement control, the moment decreased. The rigidity of measurements at two displacements appeared to converge before rod failure while exhibiting a slightly declining trend. The curvature, moment, and flexural rigidity based on on-line monitoring data are presented in Figure E.4. It can be seen that the $0.65 \mathrm{~m}^{-1}$ curvature obtained in the cyclic test is quite higher than those used in measurements as part of the experiment design. Accordingly, the flexural rigidity based on on-line monitoring was about $50 \mathrm{~N} \cdot \mathrm{m}^{2}$, a little lower than that acquired in measurements. The result agrees with the declining flexural rigidity with increasing curvature as shown in Figure E.3. Overall, the rod exhibited quite stable response prior to its final breakage.

Failure was observed in the gage section near motor 2 (left side). The failure occurred at the pellet-to-pellet interface as illustrated in Figure E.5. The end faces of two neighboring pellets were essentially quite clean. At the same time, the fracture surfaces of the cladding were serrated. Some striations had developed in the circumferential direction, but they are hardly differentiated at this magnification.

Both lateral stressed sides of the rod were found covered with equally spaced circumferential cracks throughout the gage section. Spalling only occurred at the local area near the fracture. The similar degree of damage on the lateral stressed sides exhibited by D1/ DL1 is different from that seen from unidirectional bending. The large deformation arising from unidirectional bending can produce a greater extent of damage or spalling on one side but less on the other side, as seen, for example, in S3/ Dcal (see Figure D.6). The extensive circumferential cracks revealed in D1/ DL1 did not occur in Dcal/ S3, even though the load amplitude in the latter was higher. It is thus believed that the parallel equally spaced cracks were related to a preexisting condition as D1/ DL1 actually had a thicker layer of oxidation and a higher $\mathrm{H}$ content. 


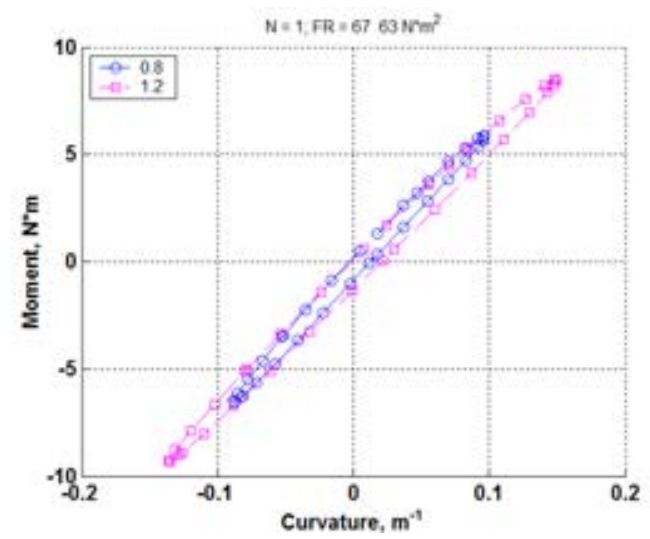

(a)

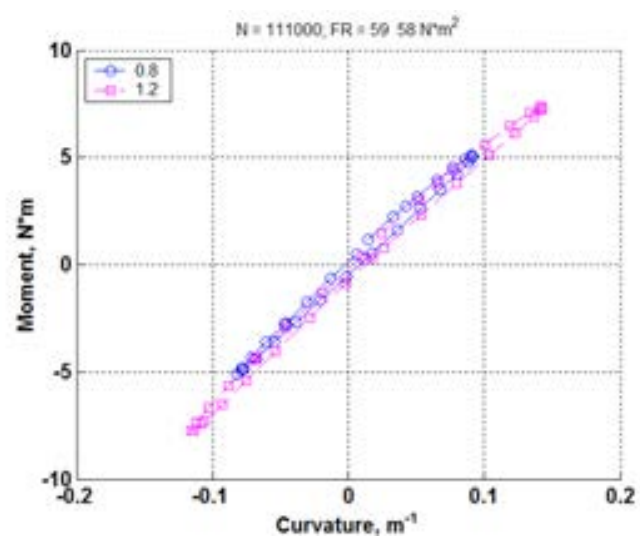

(b)

Figure E.1. Moment-curvature loops based on measurements when (a) $N=1$ and (b) $N=$ 111000 cycles for D1/ DL1 (607C4B); measurements were made with 0.8 and $1.2 \mathrm{~mm}$ relative displacements; $\mathrm{N}_{\mathrm{f}}=1.1 \times 10^{5}$ cycles under $\pm 15.24 \mathrm{~N} \cdot \mathrm{m} 5 \mathrm{~Hz}$. Fuel particles collected $<1.0$ gram.

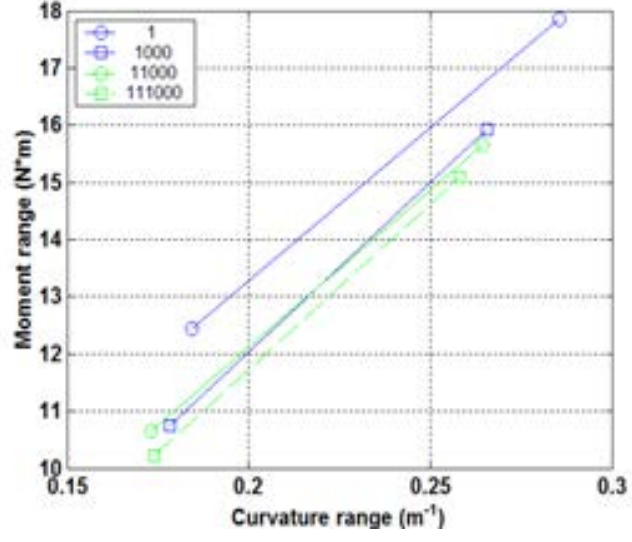

(a)

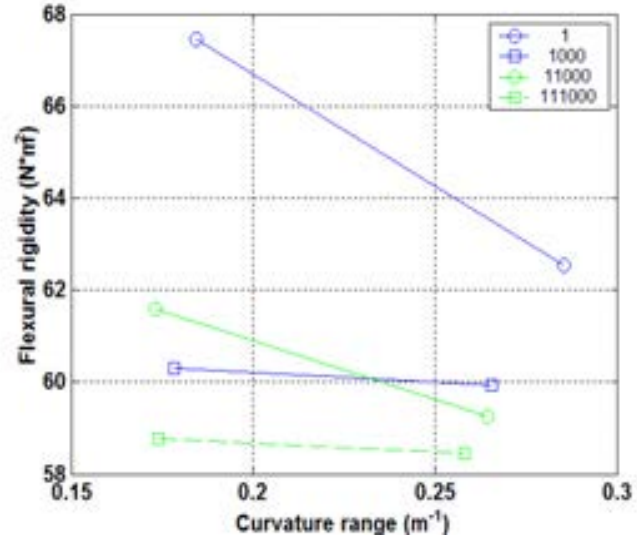

(b)

Figure E.2. (a) Moment-curvature relation and (b) moment-flexural rigidity relation at various numbers of cycles for D1/ DL1 (607C4B); $N_{f}=1.1 \times 10^{5}$ cycles under $\pm 15.24 \mathrm{~N} \cdot \mathrm{m}$ $5 \mathrm{~Hz}$. Fuel particles collected $<1.0$ gram. 


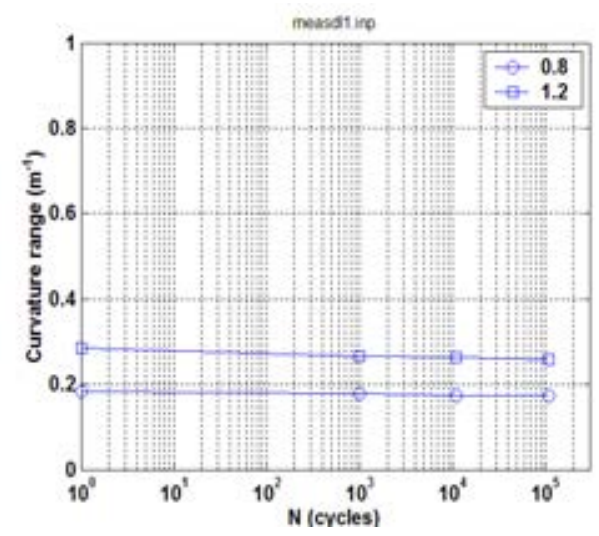

(a)

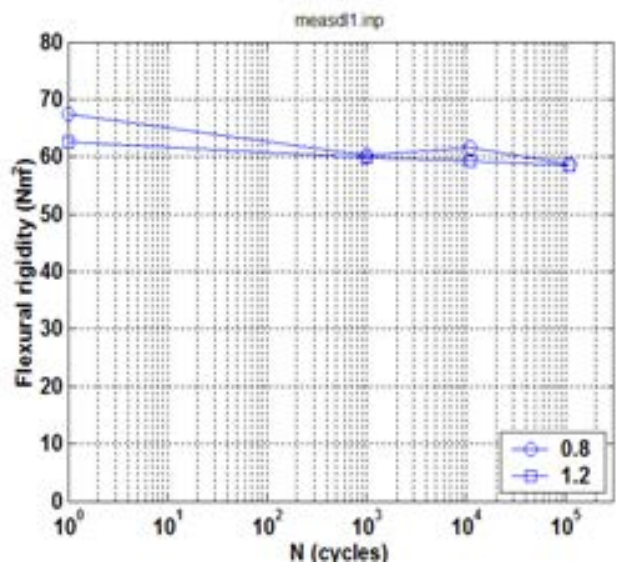

(c)

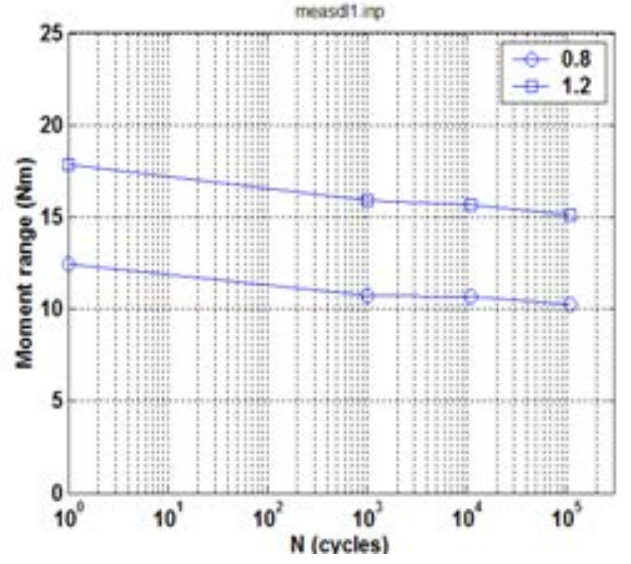

(b)

Figure E.3. Variations of (a) curvature range, (b) moment range, and (c) flexural rigidity as a function of number of cycles for D1/ DL1 (607C4B); $N_{f}=1.1 \times 10^{5}$ cycles under \pm 15.24 $\mathrm{N} \cdot \mathrm{m} 5 \mathrm{~Hz}$. Fuel particles collected $<1.0 \mathrm{gram}$. 


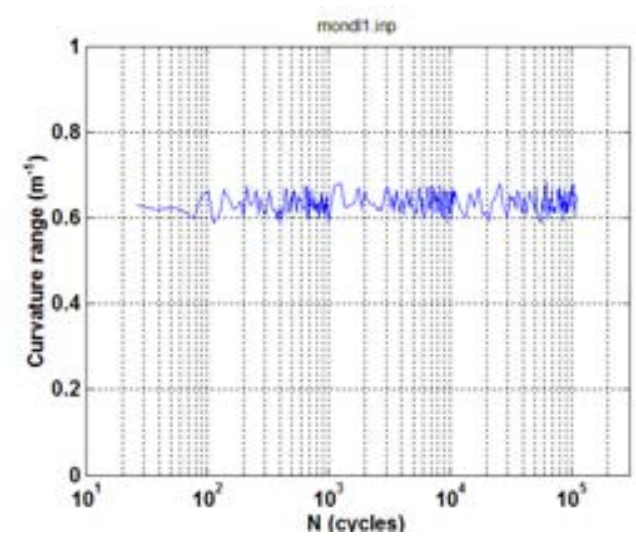

(a)

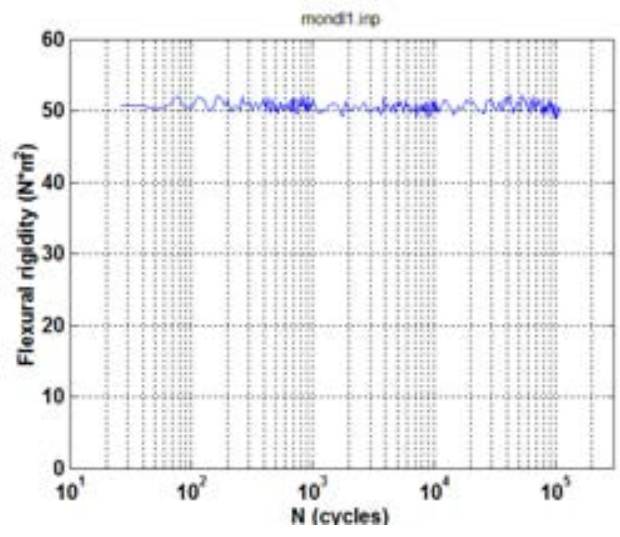

(c)

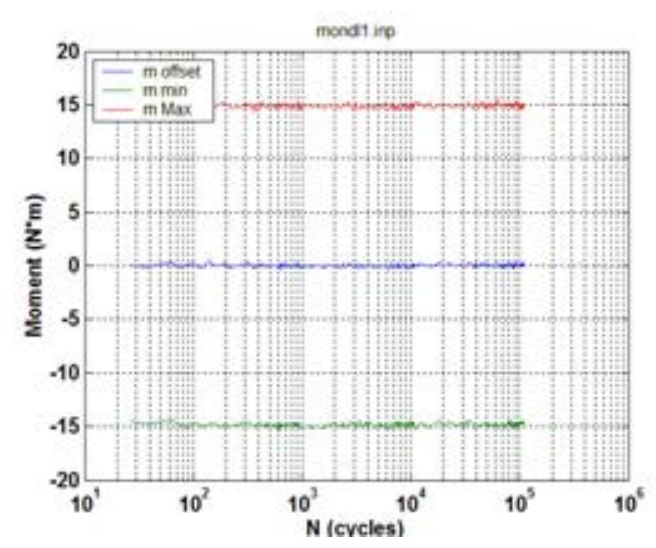

(e)

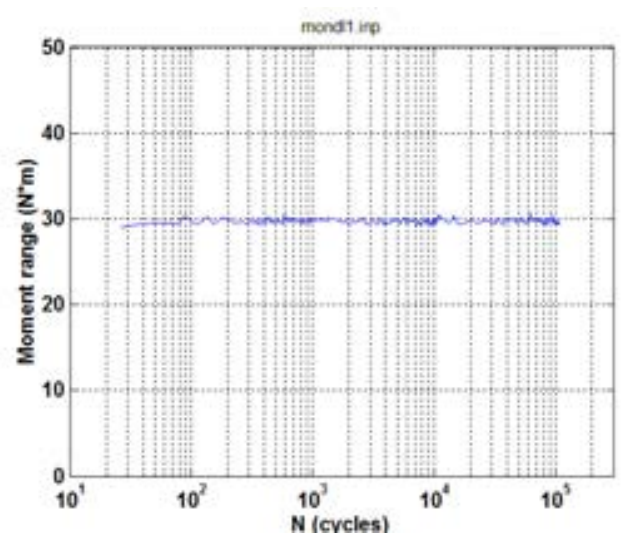

(b)

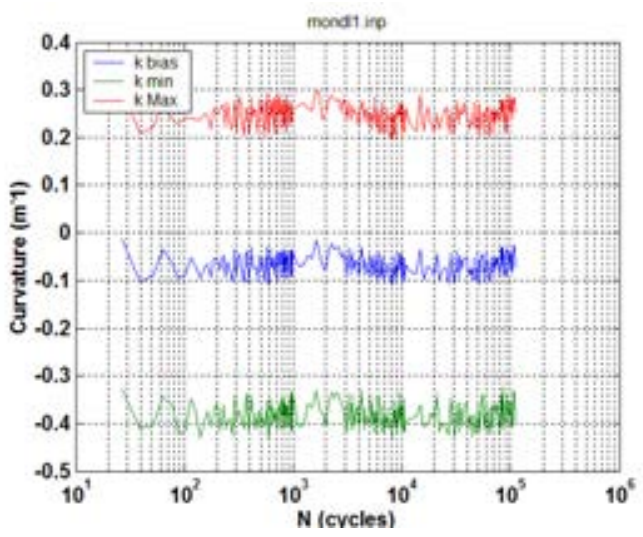

(d)

Figure E.4. Variations of (a) curvature range, (b) applied moment range, (c) flexural rigidity, (d) maximum and minimum values of curvature, and (e) maximum and minimum values of moment as a function of number of cycles for D1/ DL1 (607C4B); $\mathrm{N}_{f}=1.1 \times 10^{5}$ cycles under $\pm 15.24 \mathrm{~N} \cdot \mathrm{m} 5 \mathrm{~Hz}$. Fuel particles collected $<1.0 \mathrm{gram}$. 


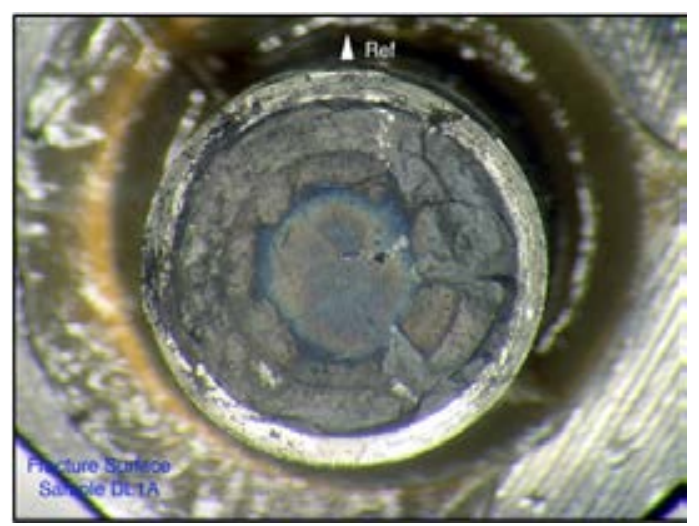

(a)
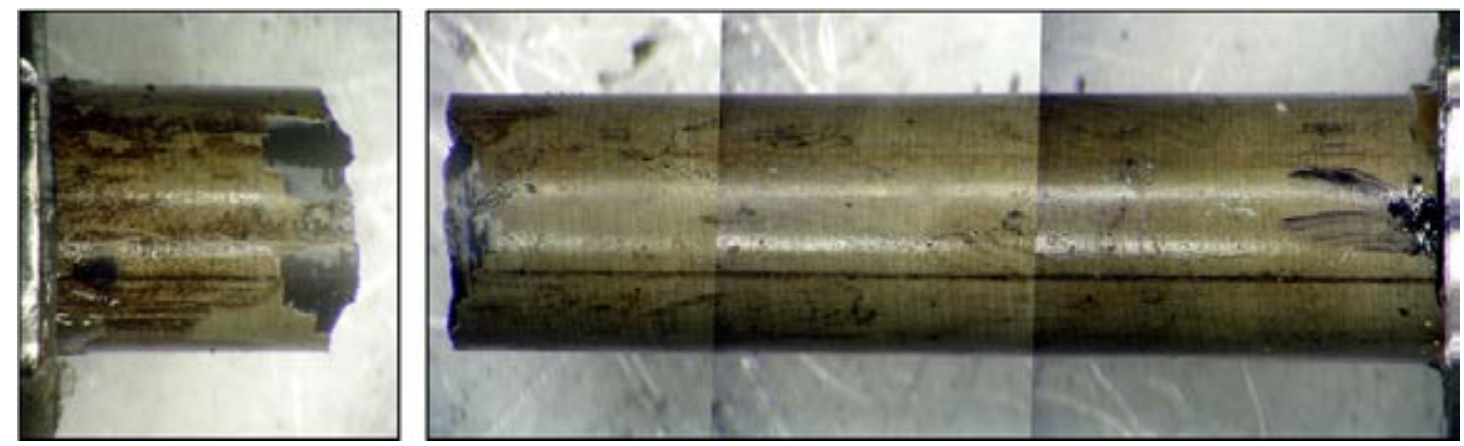

(c)
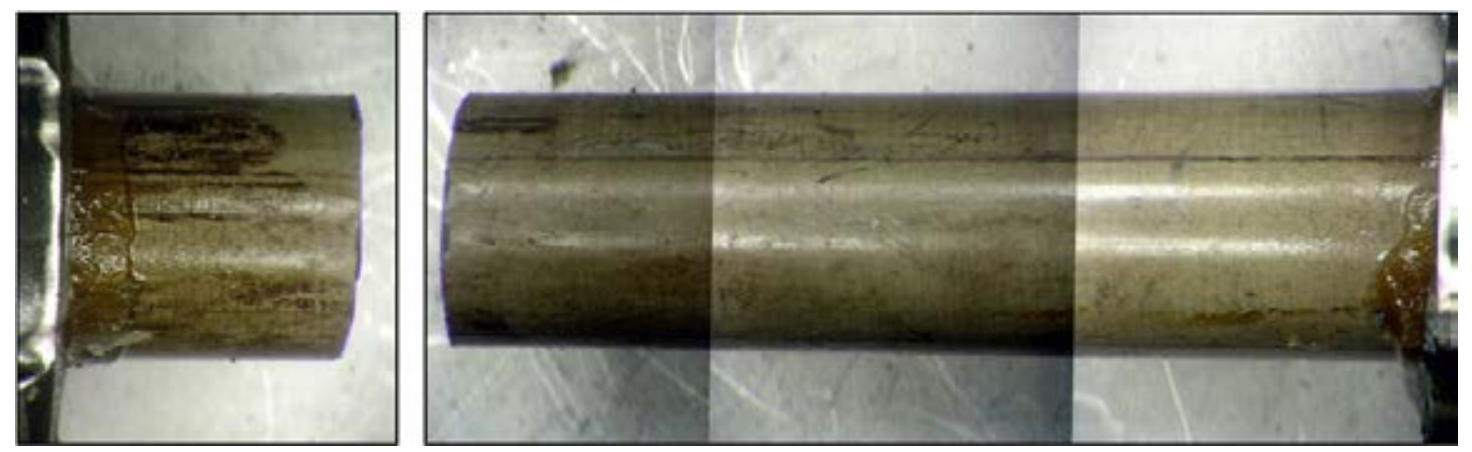

(d)

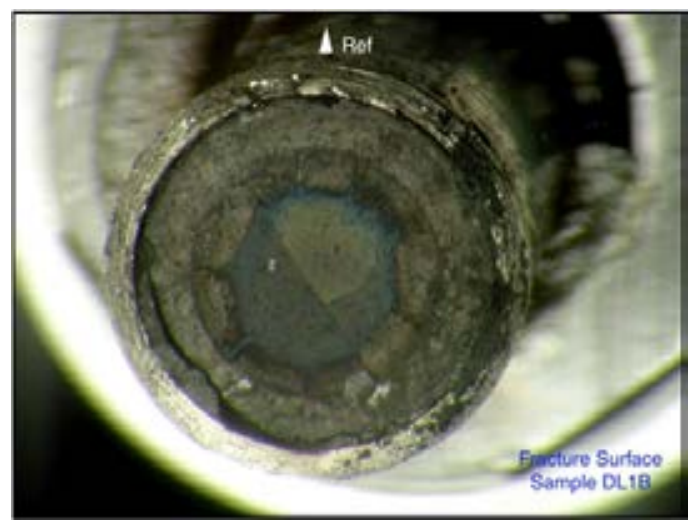

(b)

Figure E.5. (a, b) Mating fracture surfaces, (c) front side, and (d) back side of D1/ DL1 (607C4B), $N_{f}=1.1 \times 10^{5}$ cycles under $\pm 15.24 \mathrm{~N} \cdot \mathrm{m} 5 \mathrm{~Hz}$. Fuel particles collected < $1.0 \mathrm{gram}$.

\section{D2/ DL2/ 608C4B ( $\pm 5.08 / 35.56 \mathrm{~N} \cdot \mathrm{M} 5 \mathrm{HZ})$}

D2/ DL2 (608C4B, 63.8 GWd/MTU burnup, 70-100 $\mu \mathrm{m}$ oxide layer, 700 ppm H content) was tested under $\pm 5.08 \mathrm{~N} \cdot \mathrm{m} 5 \mathrm{~Hz}$. The measurement was performed using $0.4 \mathrm{~mm}$ relative displacement. The rod sustained more than $6.4 \times 10^{6}$ cycles of loading without showing any changes in flexural rigidity in both the on-line monitoring and measurements as shown in Figures E.6 and E.7 (an earlier stage of this test is not shown because the LVDT3 channel did not generate reliable data owing to loose wires). 


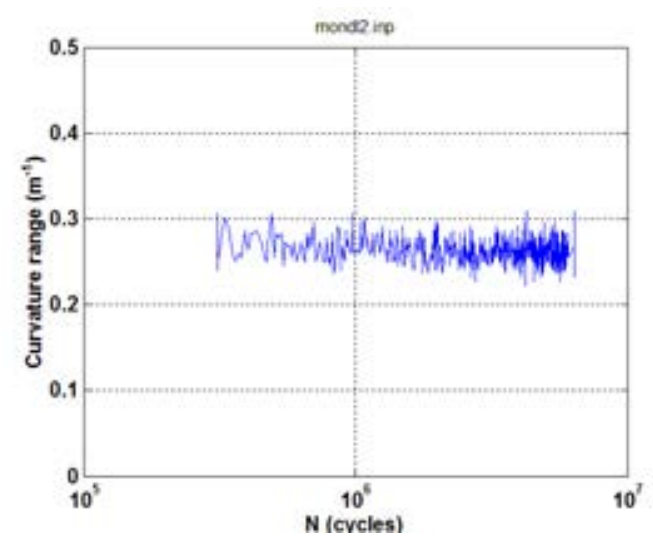

(a)

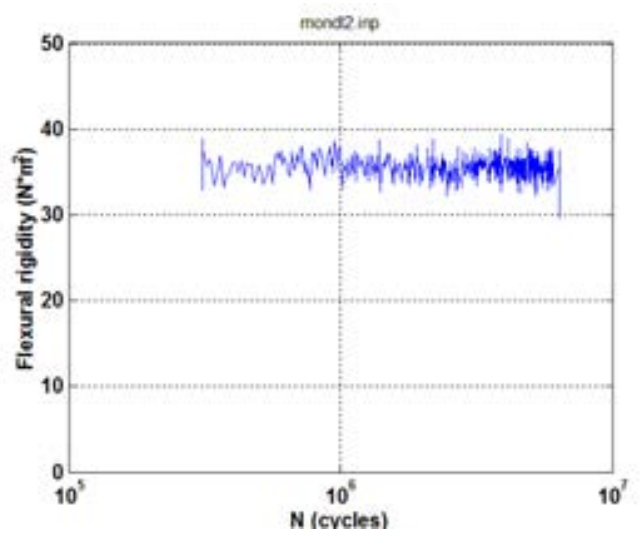

(c)

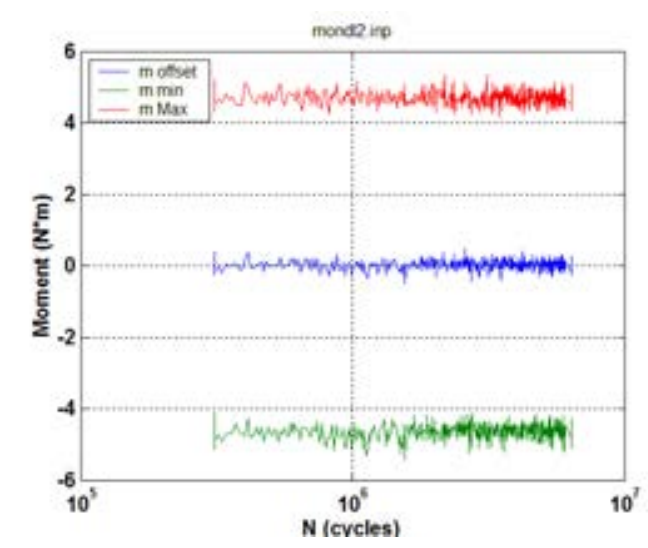

(e)

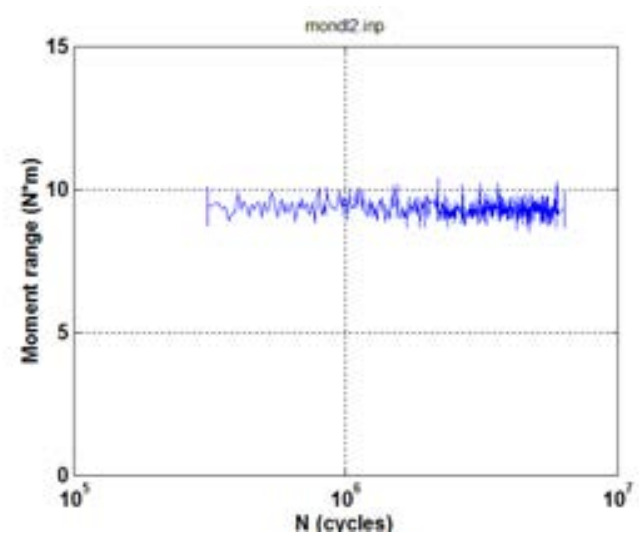

(b)

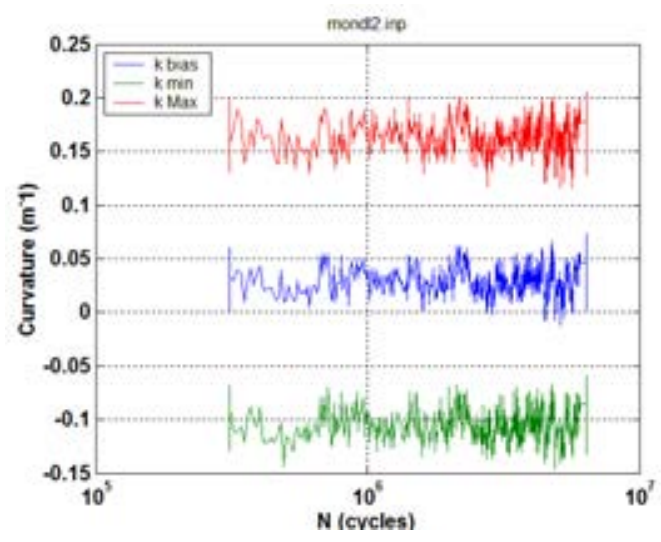

(d)

Figure E.6. Variations of (a) curvature range, (b) applied moment range, (c) flexural rigidity, (d) maximum and minimum values of curvature, and (e) maximum and minimum values of moment as a function of number of cycles for D2/ DL2 (608C4B); $6.4 \times 10^{4}$ cycles completed under $\pm 5.08 \mathrm{~N} \cdot \mathrm{m} 5 \mathrm{~Hz}$ without failure. 


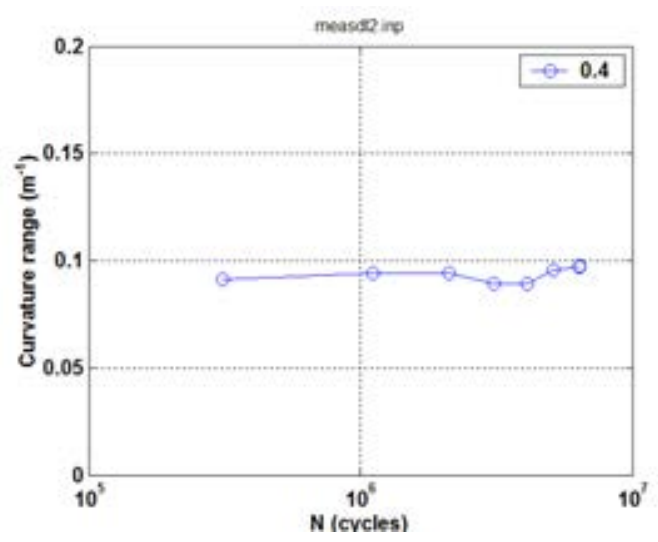

(a)

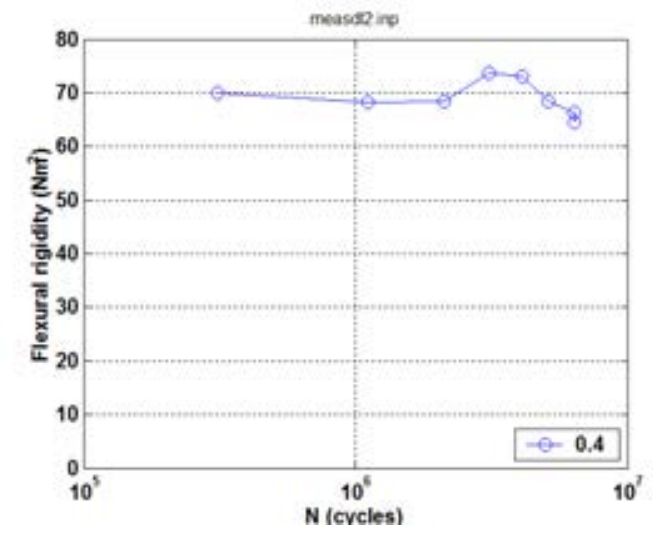

(c)

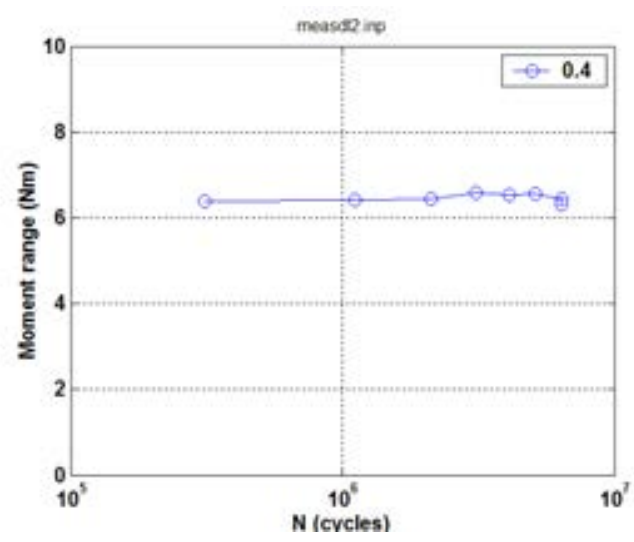

(b)

Figure E.7. Variations of (a) curvature range, (b) applied moment range, and (c) flexural rigidity as a function of number of cycles for D2/ DL2 (608C4B) based on measurements with maximum relative displacement $0.4 \mathrm{~mm} ; 6.4 \times 10^{6}$ cycles completed under $\pm 5.08 \mathrm{~N} \cdot \mathrm{m}$ $5 \mathrm{~Hz}$ without failure.

A subsequent cycle test using $\pm 35.56 \mathrm{~N} \cdot \mathrm{m} 5 \mathrm{~Hz}$ was conducted to investigate the failure mode of the tested specimen. The specimen failed near motor 2 (end cap A of the rod specimen) around $1.8 \times 10^{3}$ cycles. The clean fracture surfaces revealed that failure occurred at the pelletpellet interface (Figure E.8). The lateral view showed that the fracture surface was not a flat cut with a sharp attachment on the front side of the rod with extensive spalling. In addition, circumferential cracks covered the stressed surfaces of the rod. 


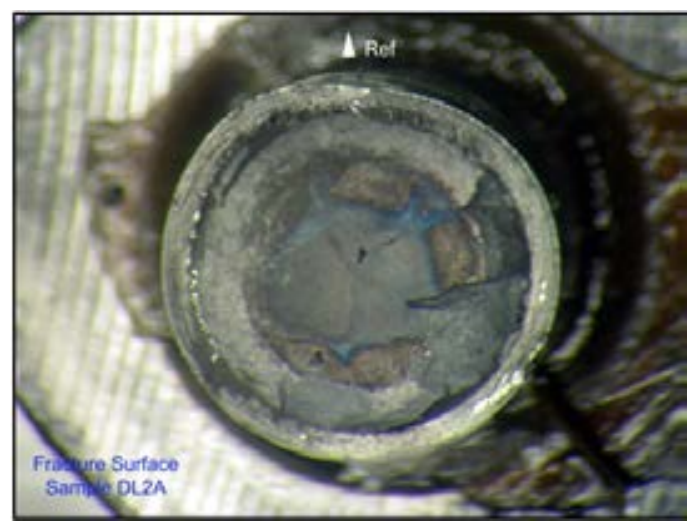

(a)

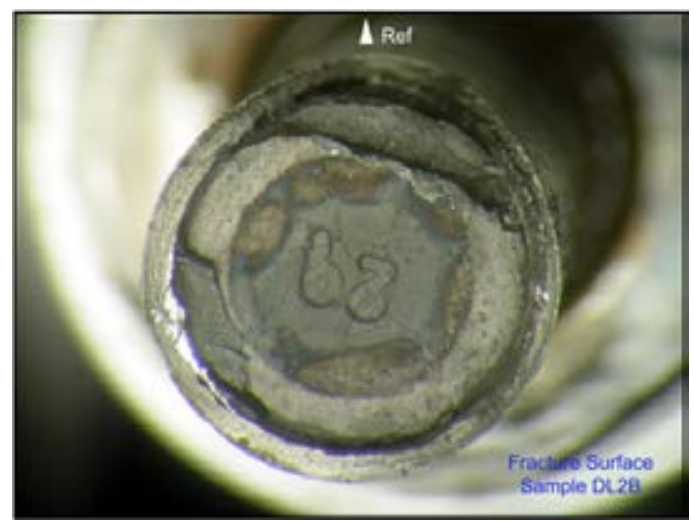

(b)
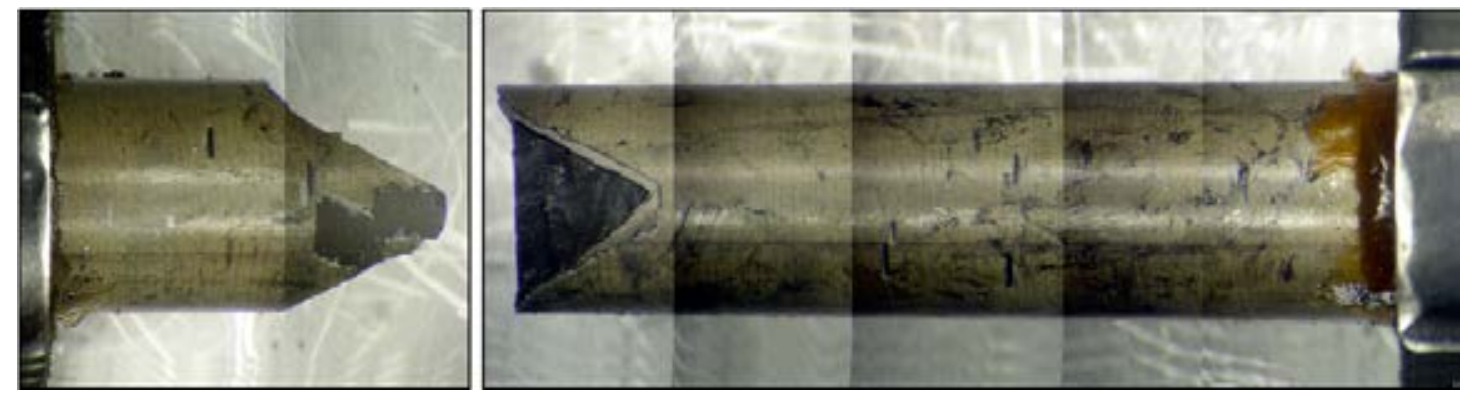

(c)
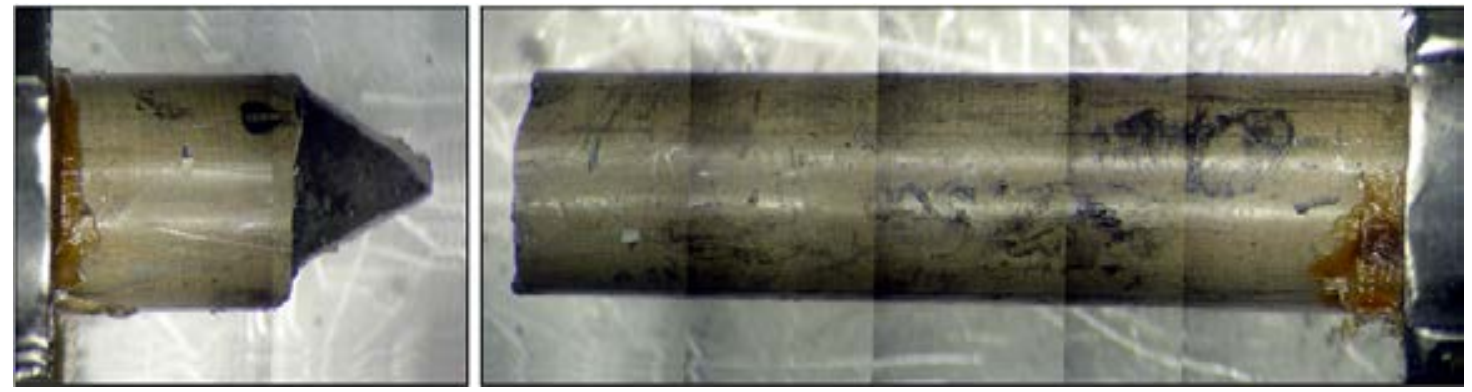

(d)

Figure E.8. (a, b) Mating fracture surfaces, (c) front side, and (d) back side of D2/ DL2 (608C4B); follow-up test was conducted with increased amplitude $\pm 35.56 \mathrm{~N} \cdot \mathrm{m} 5 \mathrm{~Hz}$ with $\mathrm{N}_{\mathrm{f}}$ $=1.8 \times 10^{3}$ cycles.

\section{D3/ DL3/ 605C10A ( $\pm 10.16 \mathrm{~N} \cdot \mathrm{M} 5 \mathrm{HZ})$}

D3/ DL3 (605C10A, 66.5 GWd/MTU burnup, 70-100 $\mu \mathrm{m}$ oxide layer, 550 ppm H content) was tested with $\pm 10.16 \mathrm{~N} \cdot \mathrm{m} 5 \mathrm{~Hz}$ at a lifetime of $1.0 \times 10^{6}$ cycles. The monitoring data revealed a slight increase in flexural rigidity prior to failure, as seen in Figure E.9. The measurements using 0.4 and $0.8 \mathrm{~mm}$ demonstrated a trend of fall and rise as shown in Figure E.10. However, the final rigidity values were still lower than the respective pre-fatigue levels. The specimen failed near motor 2 (left side, end cap A of the rod specimen). The fracture surface indicates that failure occurred at the pellet-pellet interface as the end faces of involved pellets can be seen clearly (Figure E.11). No circumferential crack was observed on the stressed surfaces of the rod. 


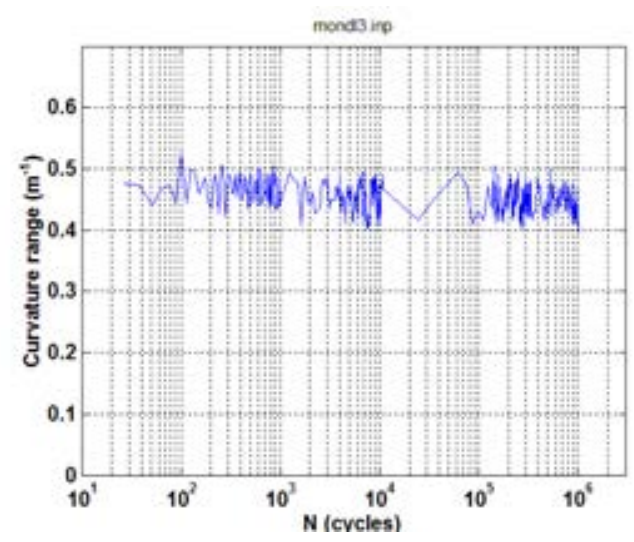

(a)

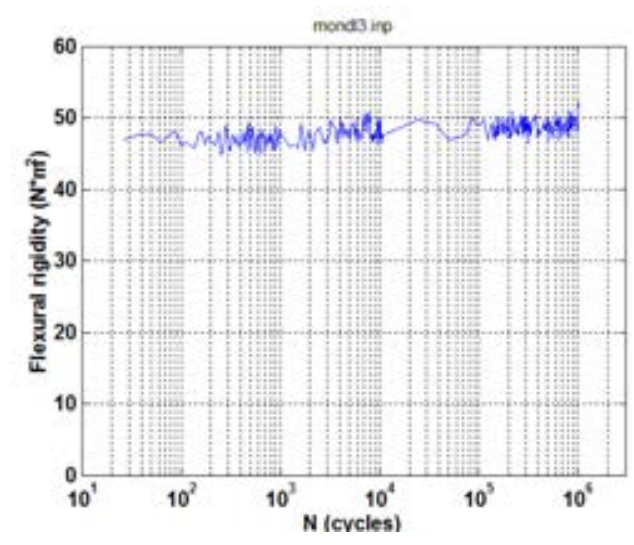

(c)

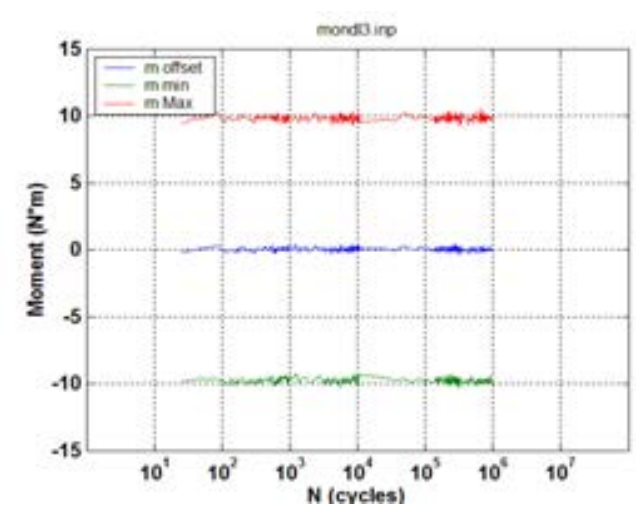

(e)

)

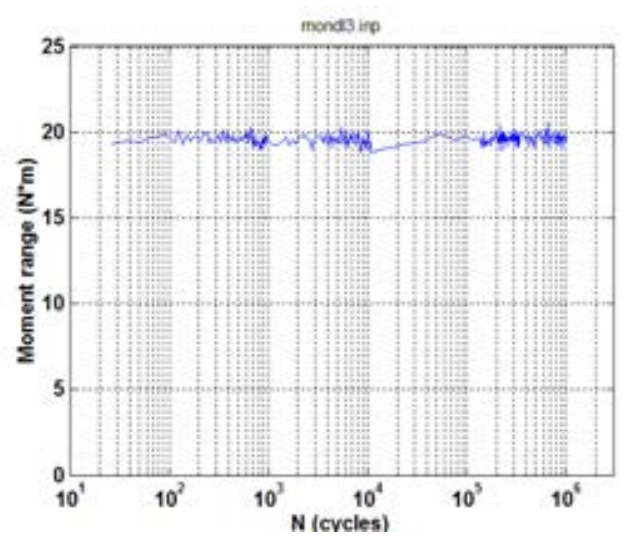

(b)

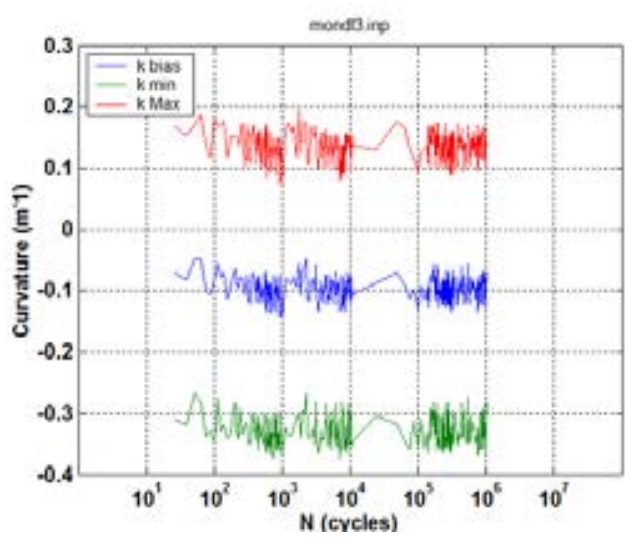

(d)

Figure E.9. Variations of (a) curvature range, (b) applied moment range, (c) flexural rigidity, (d) maximum and minimum values of curvature, and (e) maximum and minimum values of moment as a function of number of cycles for D3/ DL3 (60510A); $N_{f}=1.0 \times 10^{6}$ cycles under $\pm 10.60 \mathrm{~N} \cdot \mathrm{m} 5 \mathrm{~Hz}$. 


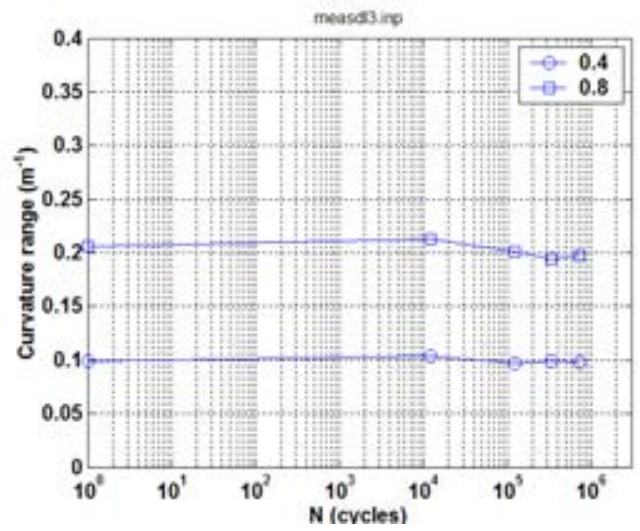

(a)

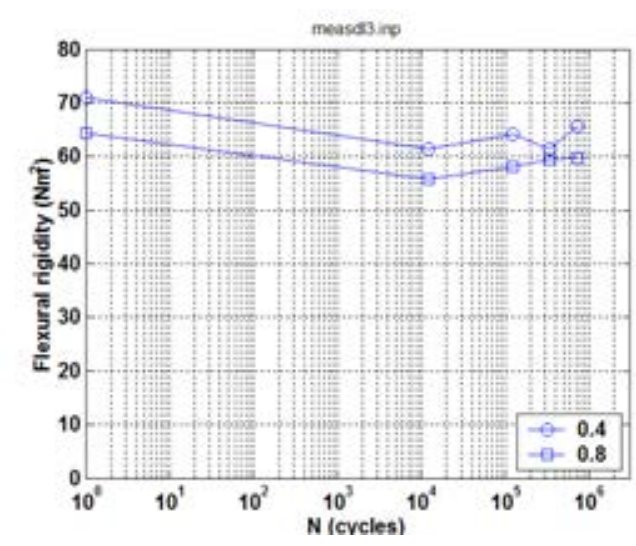

(c)

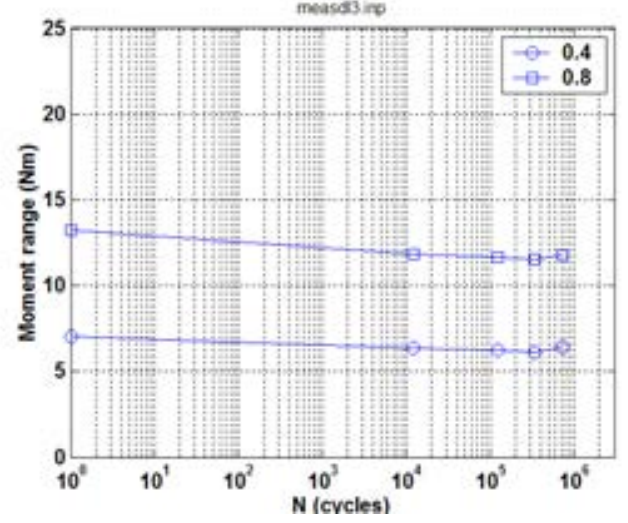

(b)

Figure E.10. Variations of (a) curvature range, (b) applied moment range, (c) flexural rigidity as a function of number of cycles for D3/ DL3 (60510A) based on measurements with maximum relative displacement at 0.4 and $0.8 \mathrm{~mm} ; \mathrm{N}_{\mathrm{f}}=1.0 \times 10^{6}$ cycles under \pm 10.16 $\mathrm{N} \cdot \mathrm{m} 5 \mathrm{~Hz}$. 


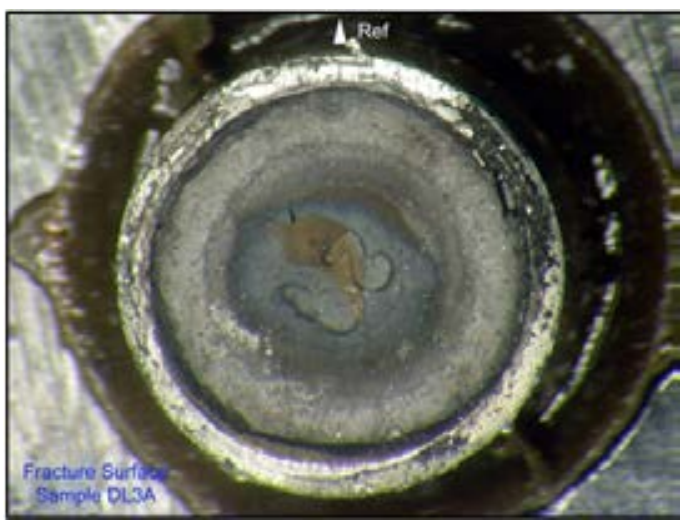

(a)

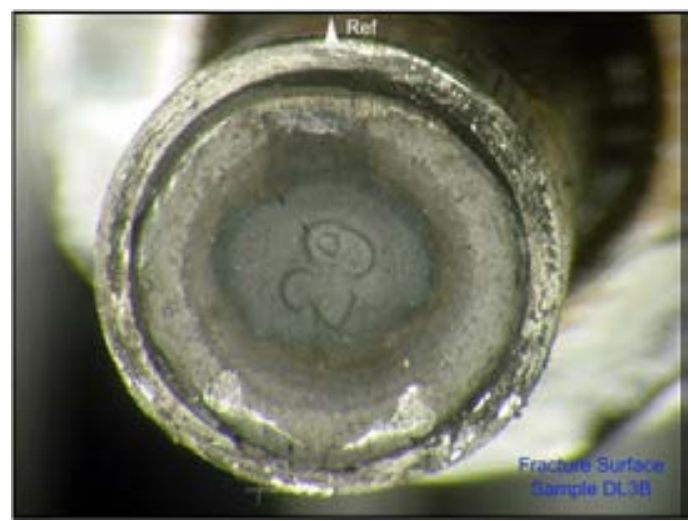

(b)
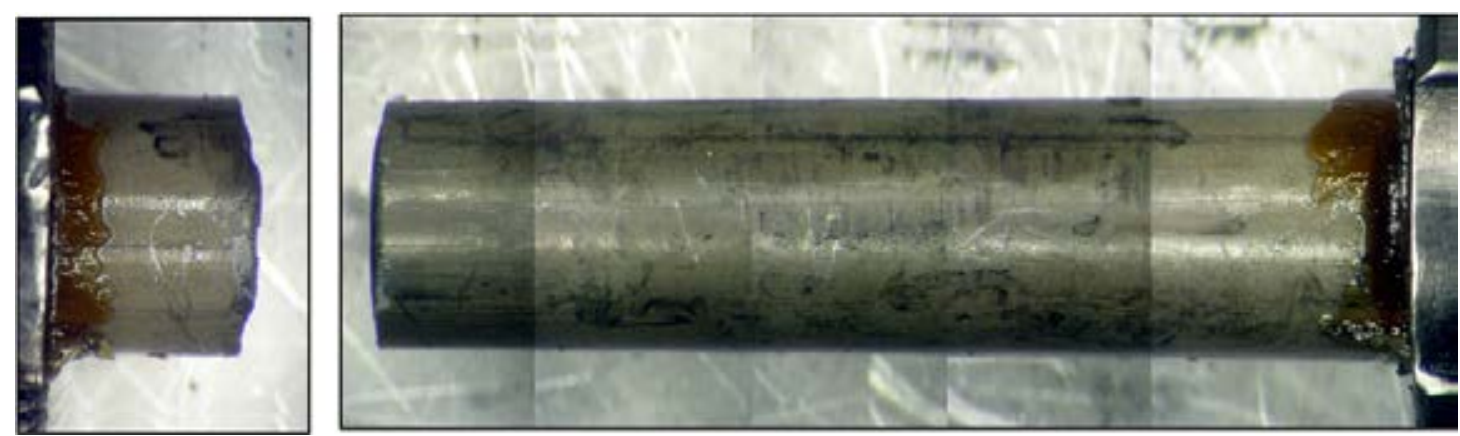

(c)
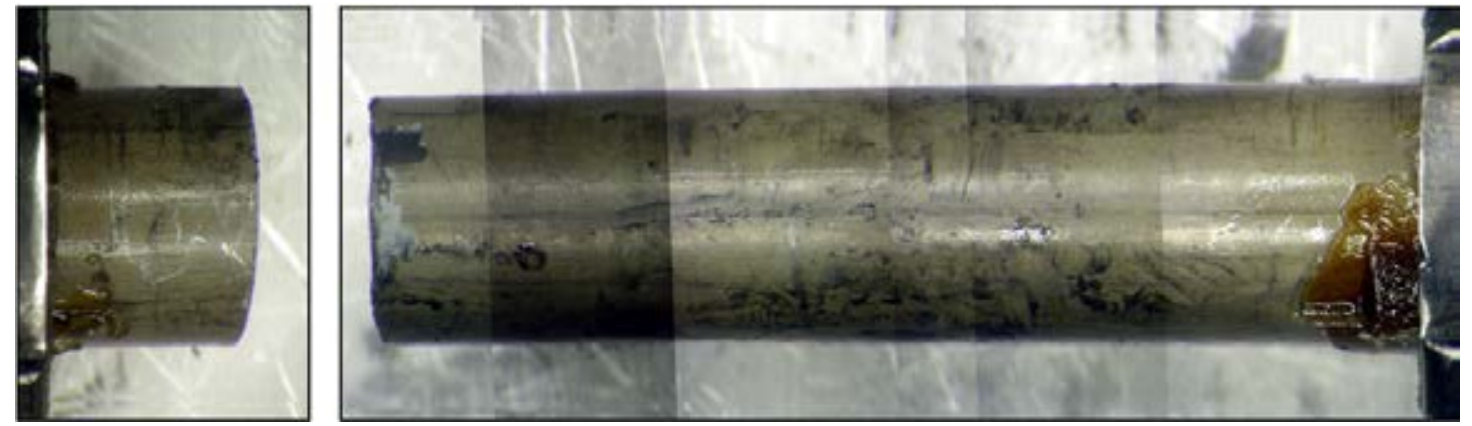

(d)

Figure E.11. (a, b) Mating fracture surfaces, (c) front side, and (d) back side of D3/ DL3 $(605 \mathrm{C} 10 \mathrm{~A}) ; \mathrm{N}_{\mathrm{f}}=1.0 \times 10^{6}$ cycles under $\pm 10.16 \mathrm{~N} \cdot \mathrm{m} 5 \mathrm{~Hz}$.

\section{D4/ DM1/ 605D1C ( $\pm 7.62 \mathrm{~N} \cdot \mathrm{M} 5 \mathrm{HZ})$}

D4/ DM1 (605D1C, 66.5 GWd/MTU burnup, 40-70 $\mu \mathrm{m}$ oxide layer, 500 ppm H content) was tested under $\pm 7.62 \mathrm{~N} \cdot \mathrm{m} 5 \mathrm{~Hz}$ as the result of an effort to define a possible fatigue limit for the SNF rod. DM1 finished the target of $1.1 \times 10^{7}$ cycles without any sign of significant fatigue during the cyclic test, and the test was stopped.

On-line monitoring demonstrated that the flexural rigidity fluctuated between 42 and $49 \mathrm{~N} \cdot \mathrm{m}^{2}$ with a marginal decrease near the end of the test (Figure E.12). The measurements using 0.4 
and $0.6 \mathrm{~mm}$ also demonstrated a certain amount of drop in rigidity near the end-of-cycle test, as shown in Figure E.13.

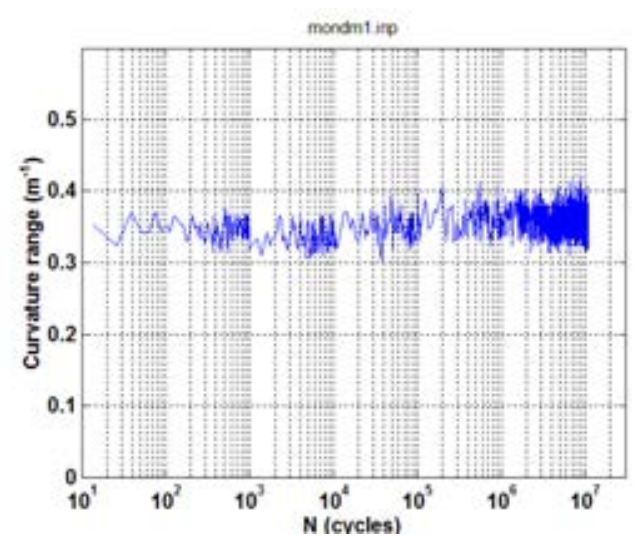

(a)

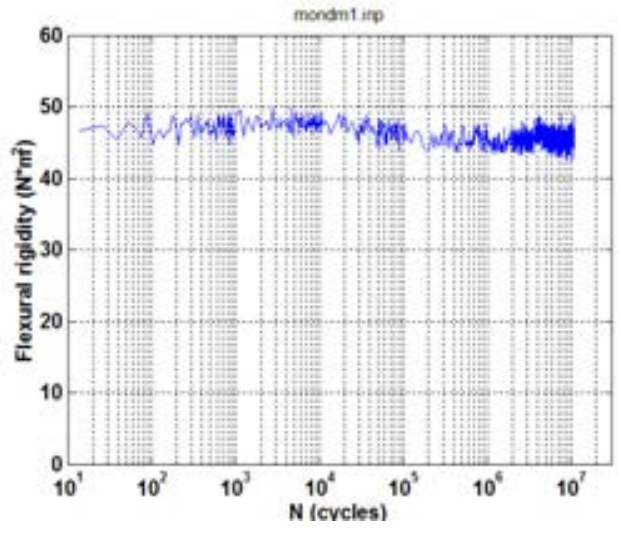

(c)

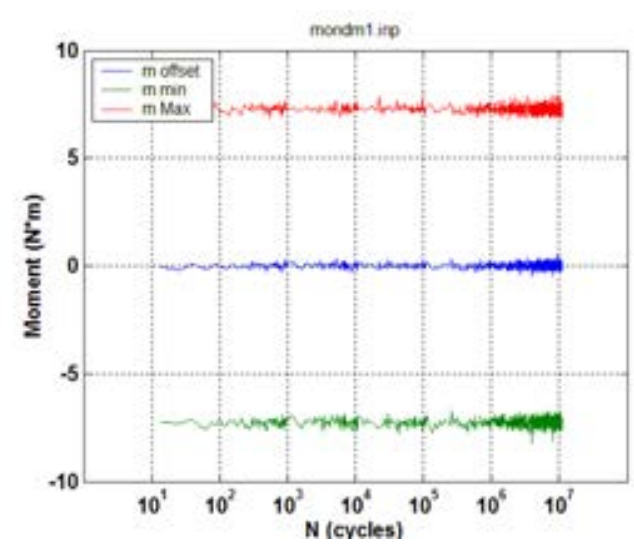

(e)

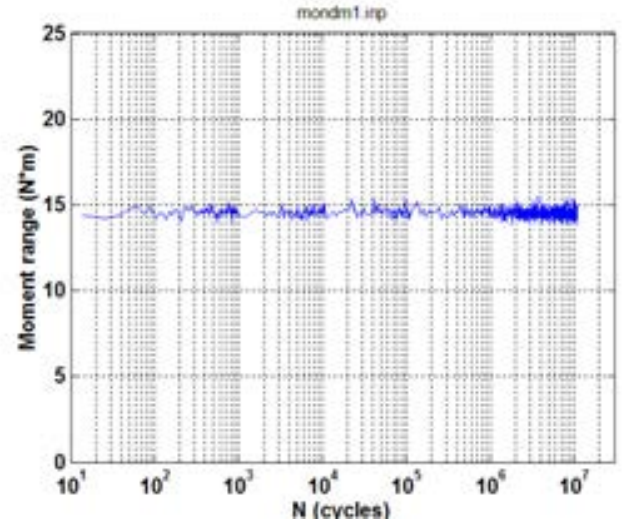

(b)

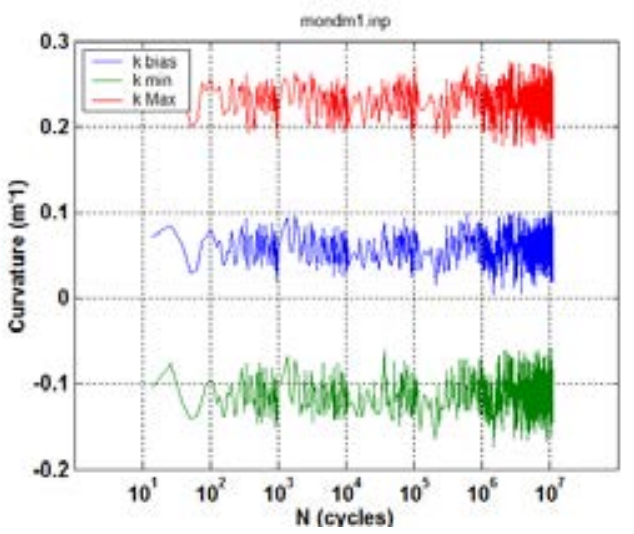

(d) 


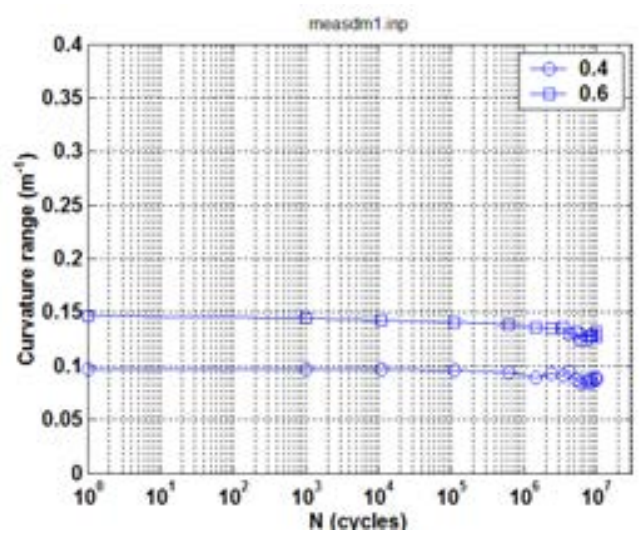

(a)

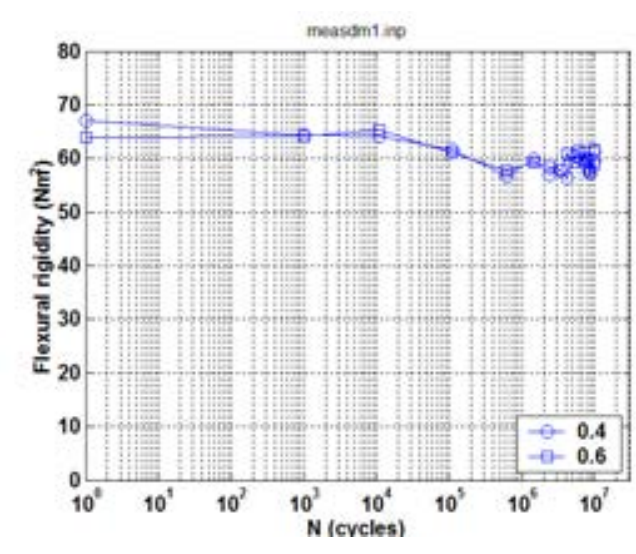

(c)

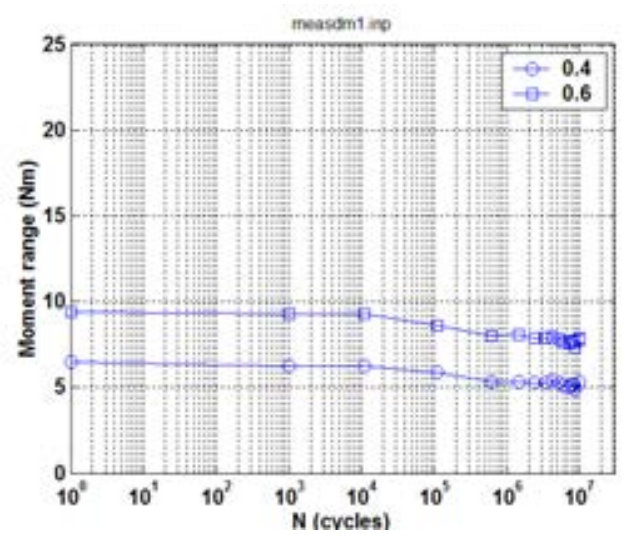

(b)

(b)

Figure E.13. Variations of (a) curvature range, (b) applied moment range, and (c) flexural rigidity as a function of number of cycles for D4/ DM1 (605D1C) based on measurements with maximum relative displacement at 0.4 and $0.6 \mathrm{~mm} ; \mathrm{N}=1.0 \times 10^{7}$ cycles under $\pm 7.62 \mathrm{~N} \cdot \mathrm{m} 5 \mathrm{~Hz}$.

\section{D5/ DM2/ 605D1B ( $\pm 9.14 \mathrm{~N} \cdot \mathrm{M} 5 \mathrm{HZ})$}

The cycle test on D5/ DM2 (605D1B, $66.5 \mathrm{GWd} / \mathrm{MTU}$ burnup, 40-70 $\mu \mathrm{m}$ oxide layer, $550 \mathrm{ppm} \mathrm{H}$ content) was conducted under $\pm 9.14 \mathrm{~N} \cdot \mathrm{m} 5 \mathrm{~Hz}$. DM2 fractured near $2.3 \times 10^{6}$ cycles.

On-line monitoring demonstrated that the flexural rigidity fluctuated between 47 and $51 \mathrm{~N} \cdot \mathrm{m}^{2}$ with a marginal decrease near the end of test (Figure E.14). The measurements using 0.4 and $0.8 \mathrm{~mm}$ also showed a similar degree of drop in rigidity near the end of the test, as shown in Figure E.15.

DM2 fractured in the gage section near motor 2 (left side) as shown in Figure E.16. The involvement of the pellet-pellet interface was not quite clear because no end face of a pellet can be identified. No circumferential cracking can be seen on either of the stressed surfaces of the rod, but spalling was observed near the fracture. 


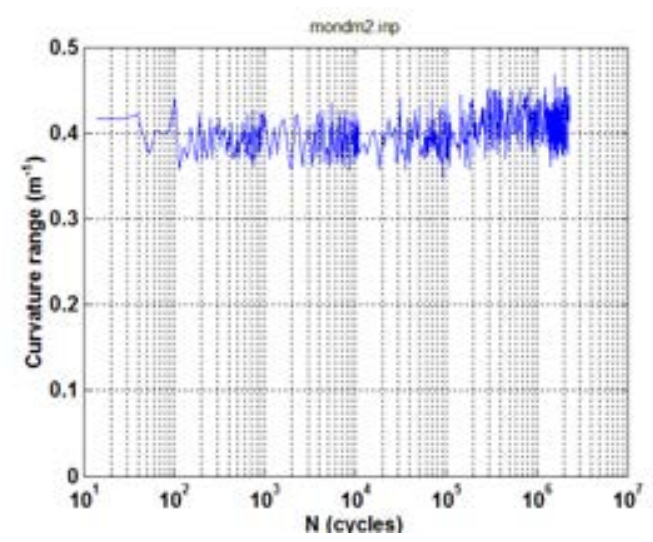

(a)

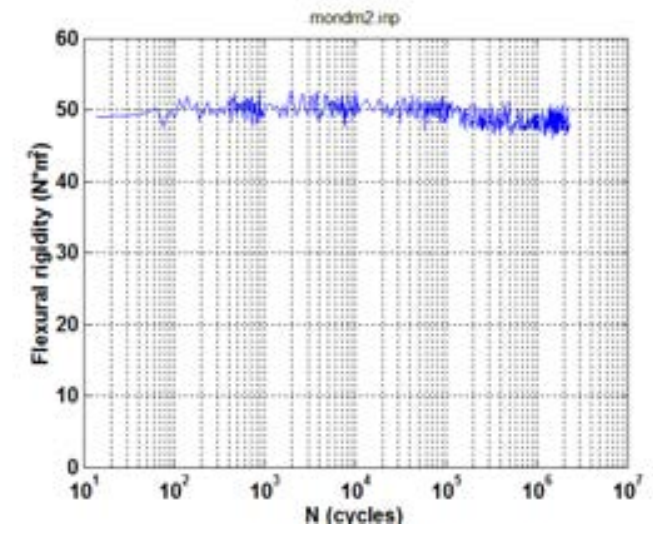

(c)

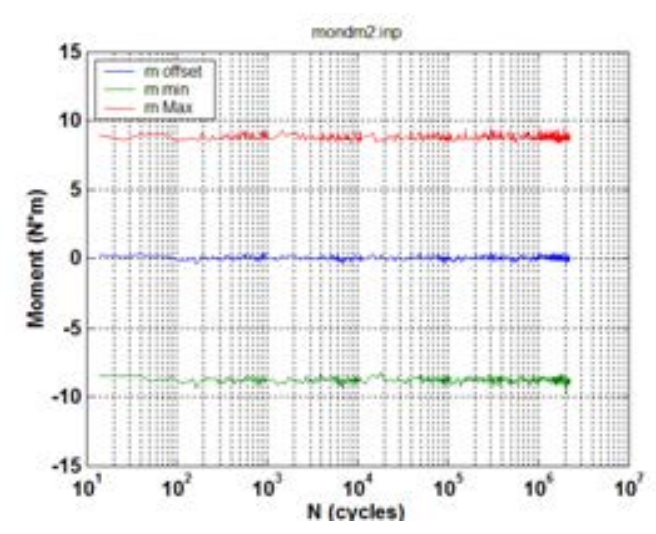

(e)

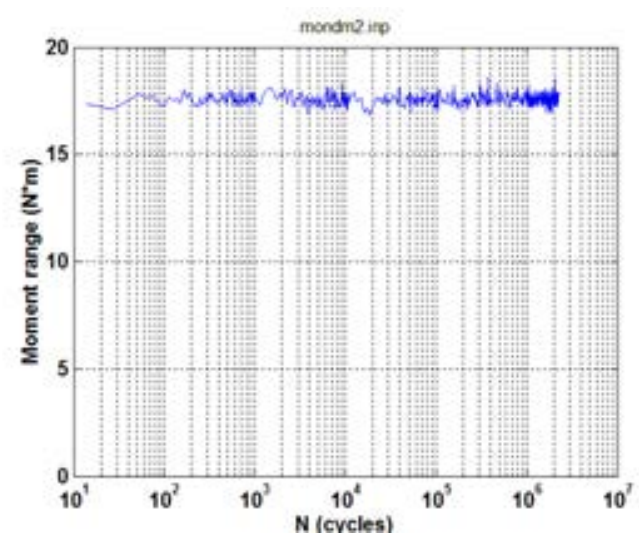

(b)

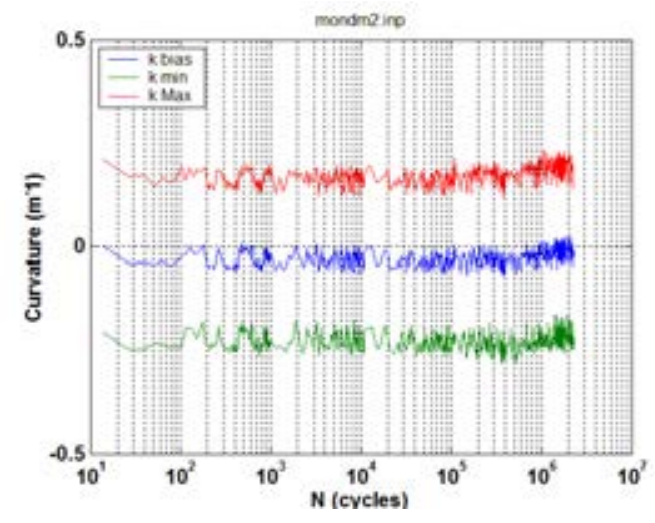

(d)

(d)

Figure E.14. Variations of (a) curvature range, (b) applied moment range, (c) flexural rigidity, (d) maximum and minimum values of curvature, and (e) maximum and minimum values of moment as a function of number of cycles for D5/ DM2 (605D1B); $N_{f}=2.3 \times 10^{6}$ cycles under $\pm 9.14 \mathrm{~N} \cdot \mathrm{m} 5 \mathrm{~Hz}$. 


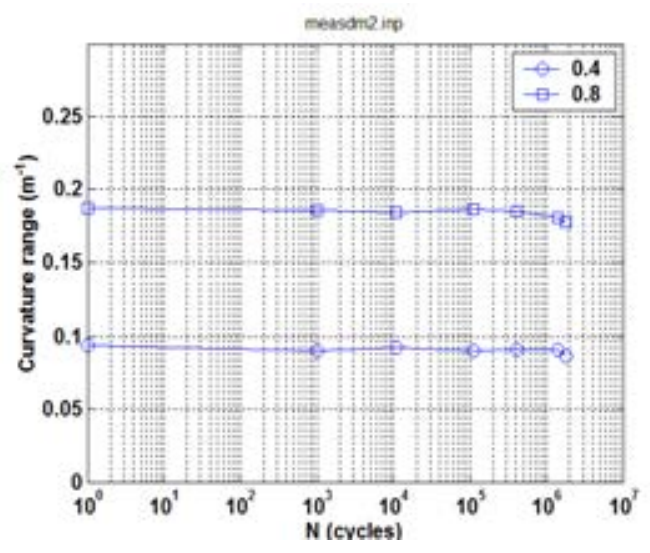

(a)

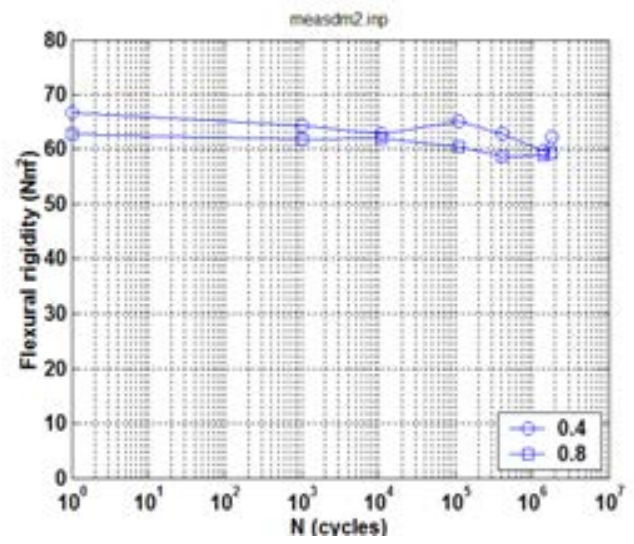

(c)

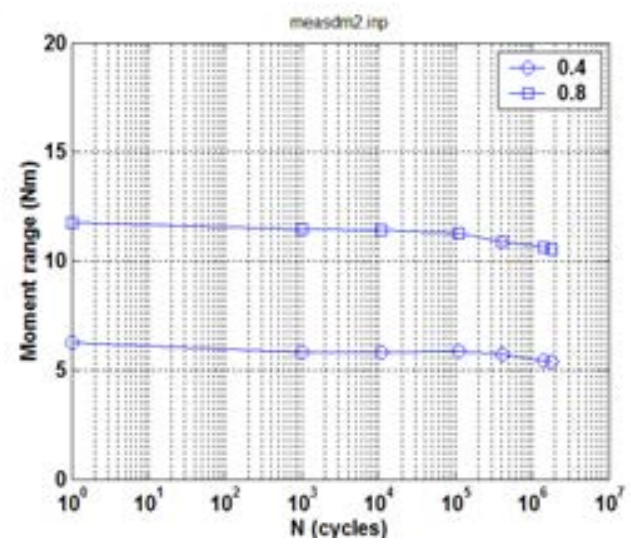

(b)

Figure E.15. Variations of (a) curvature range, (b) applied moment range, and (c) flexural rigidity as a function of number of cycles for D5/ DM2 (605D1B) based on measurements with maximum relative displacement at 0.4 and $0.8 \mathrm{~mm} ; \mathrm{N}_{\mathrm{f}}=2.3 \times 10^{6}$ cycles under \pm 9.14 $\mathrm{N} \cdot \mathrm{m} 5 \mathrm{~Hz}$. 


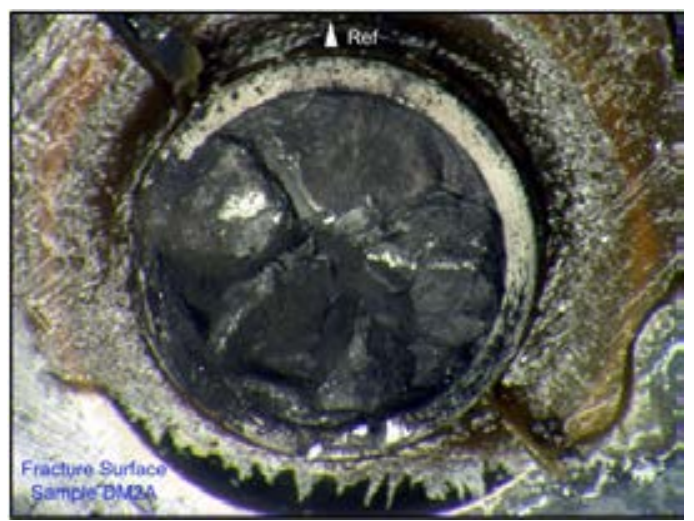

(a)

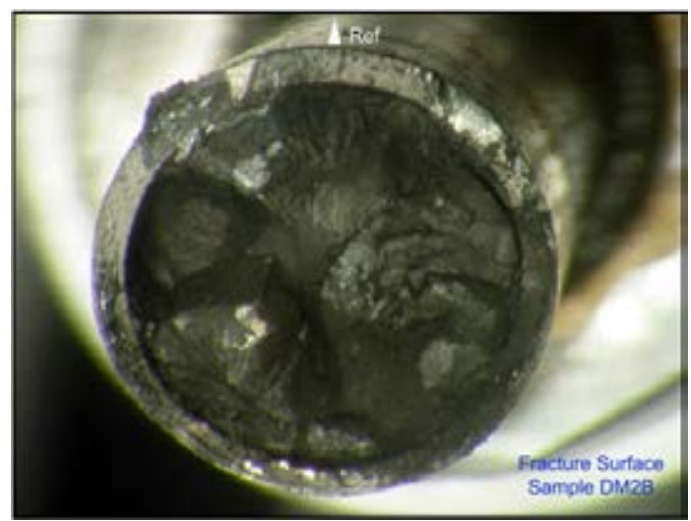

(b)
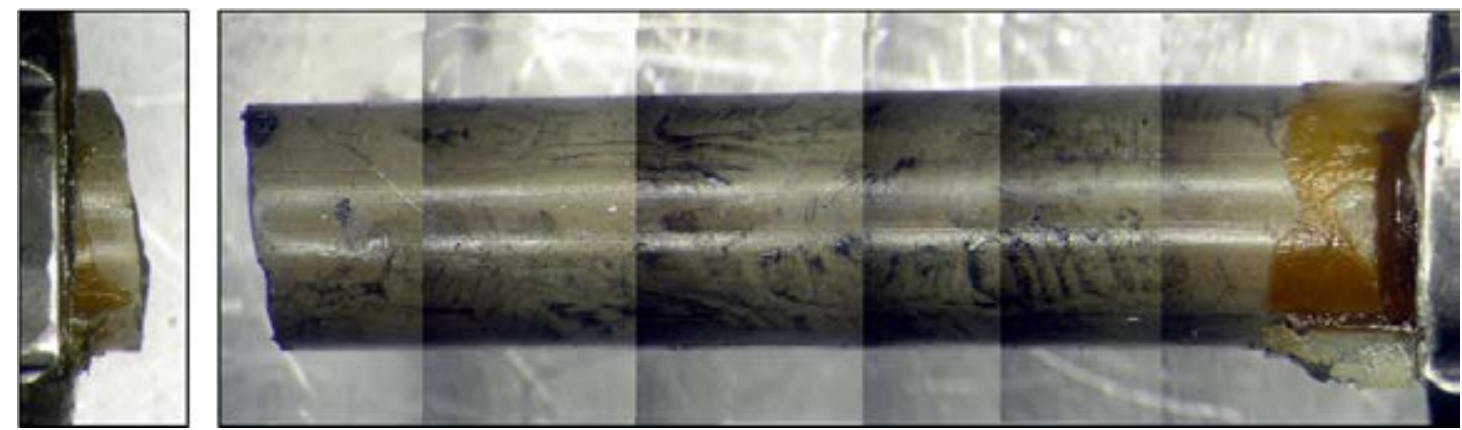

(c)
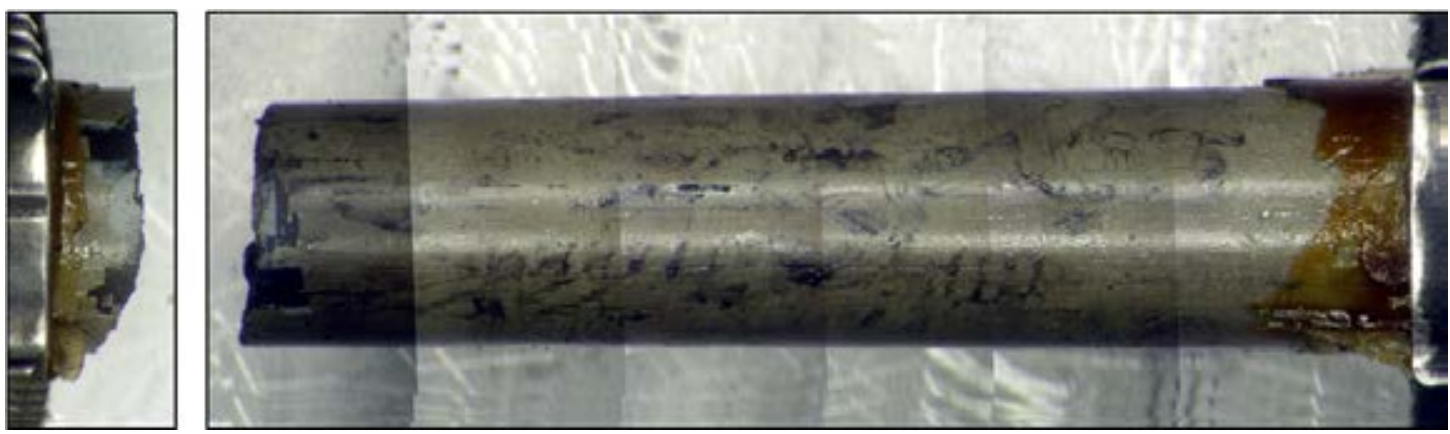

(d)

Figure E.16. (a, b) Mating fracture surfaces, (c) front side, and (d) back side of D5/ DM2 (605D1B); $\mathrm{N}_{\mathrm{f}}=2.3 \times 10^{6}$ cycles under $\pm 9.14 \mathrm{~N} \cdot \mathrm{m} 5 \mathrm{~Hz}$.

\section{D6/ DH1/ 609C4 ( $\pm 12.7 \mathrm{~N} \cdot \mathrm{M} 5 \mathrm{HZ})$}

The cycle test on D6/ DH1 (609C4, 66.5 GWd/MTU burnup, 70-100 $\mu \mathrm{m}$ oxide layer, $700 \mathrm{ppm} \mathrm{H}$ content) was conducted under $\pm 12.7 \mathrm{~N} \cdot \mathrm{m} 5 \mathrm{~Hz}$. D6/ DH1 fractured around $2.4 \times 10^{5}$ cycles.

On-line monitoring demonstrated that the flexural rigidity remained between 42 and $45 \mathrm{~N} \cdot \mathrm{m}^{2}$ over the cycle test with a slight increase (Figure E.17Figure E.17). The measurements using 0.4 and $0.8 \mathrm{~mm}$ also showed a similar variation in rigidity as shown in Figure E.18. 
$\mathrm{DH} 1$ fractured in the gage section near motor 2 (left side) as shown in Figure E.19. The involvement of the pellet-pellet interface was not quite clear because no end face of a pellet can be seen. However, equally spaced circumferential cracks exist on both stressed surfaces of the rod.

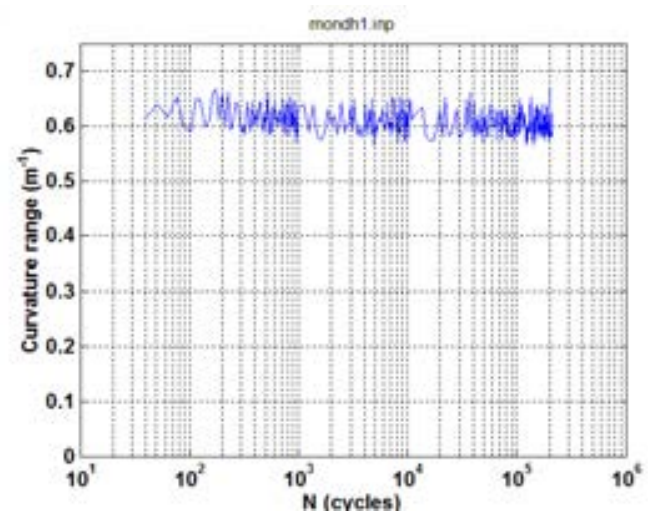

(a)

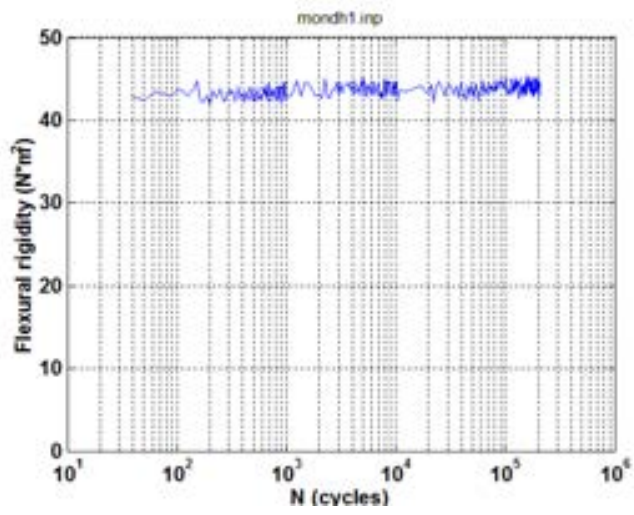

(c)

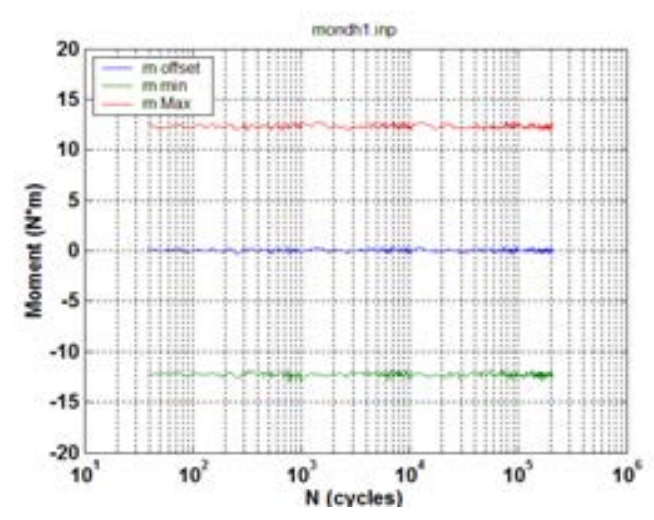

(e)

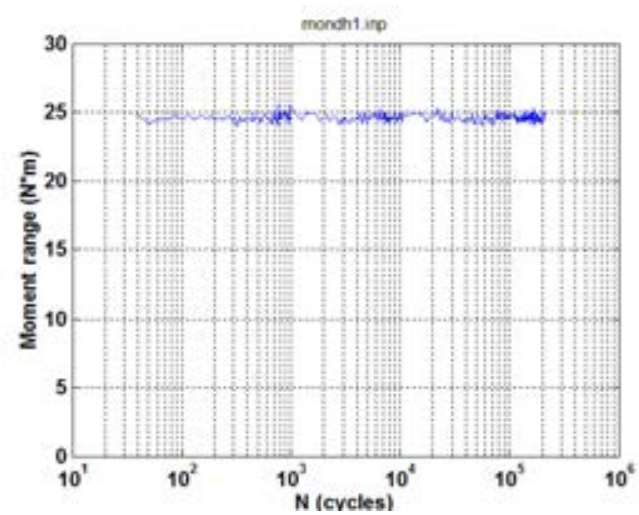

(b)

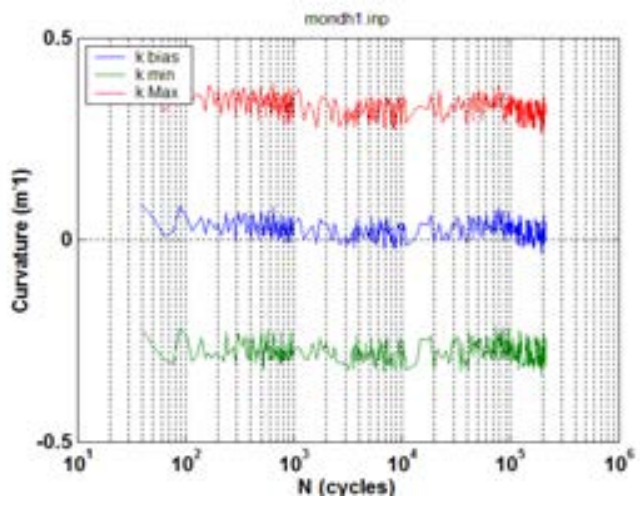

(d)

\section{)}




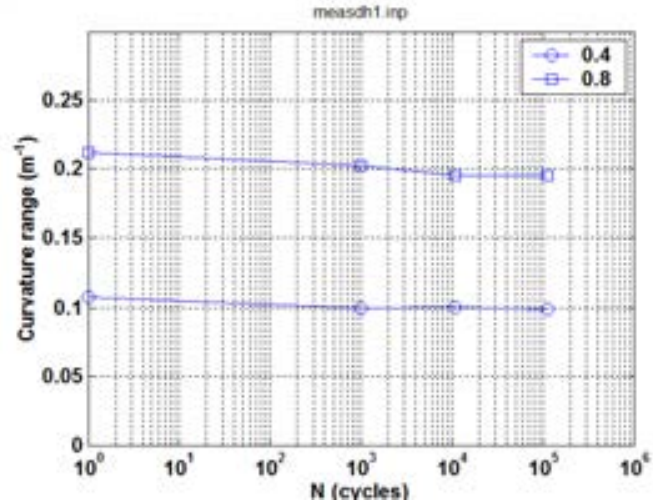

(a)

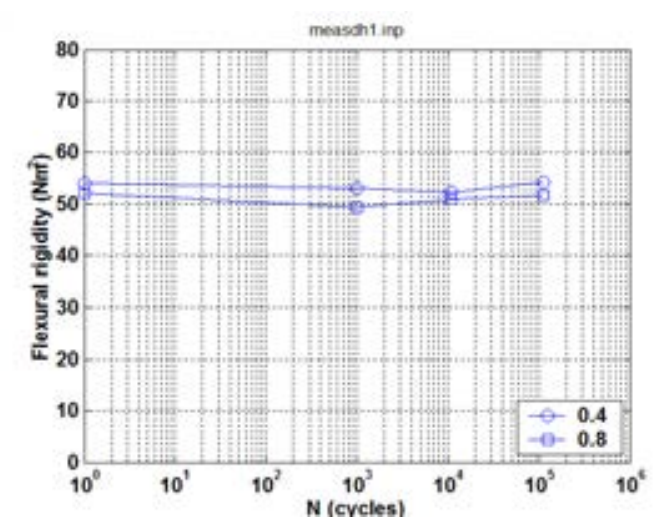

(c)

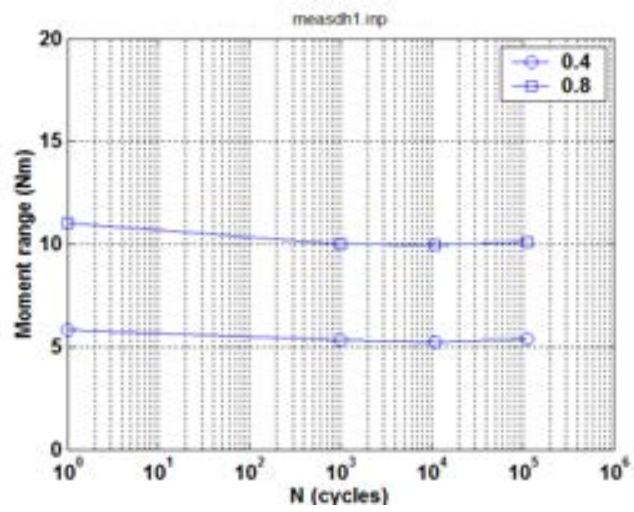

(b)

(b) 


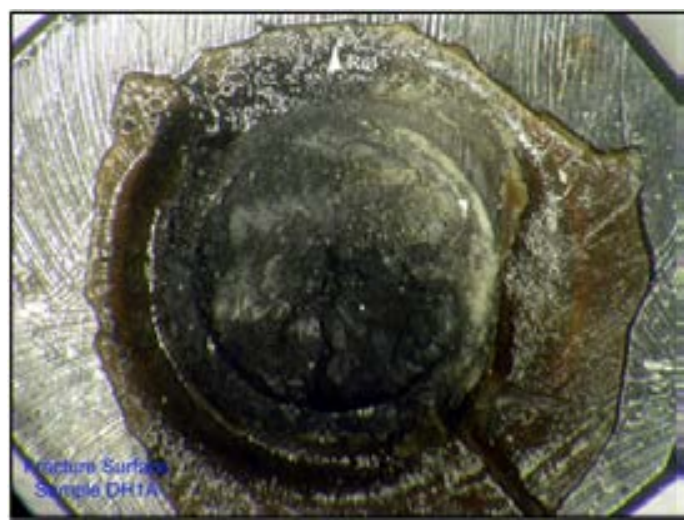

(a)

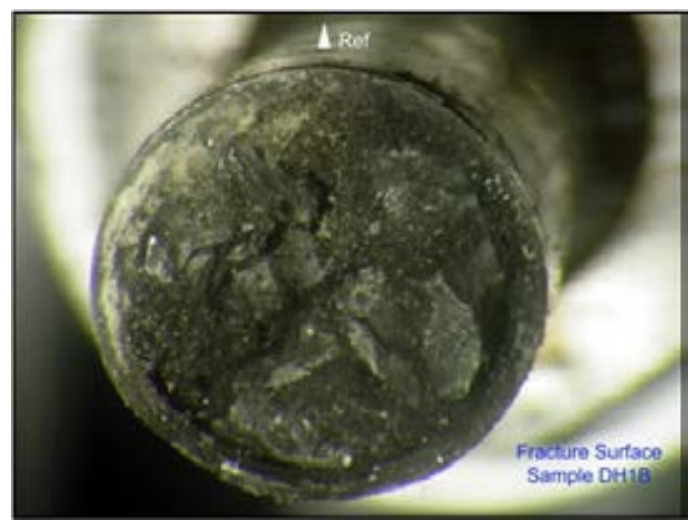

(b)
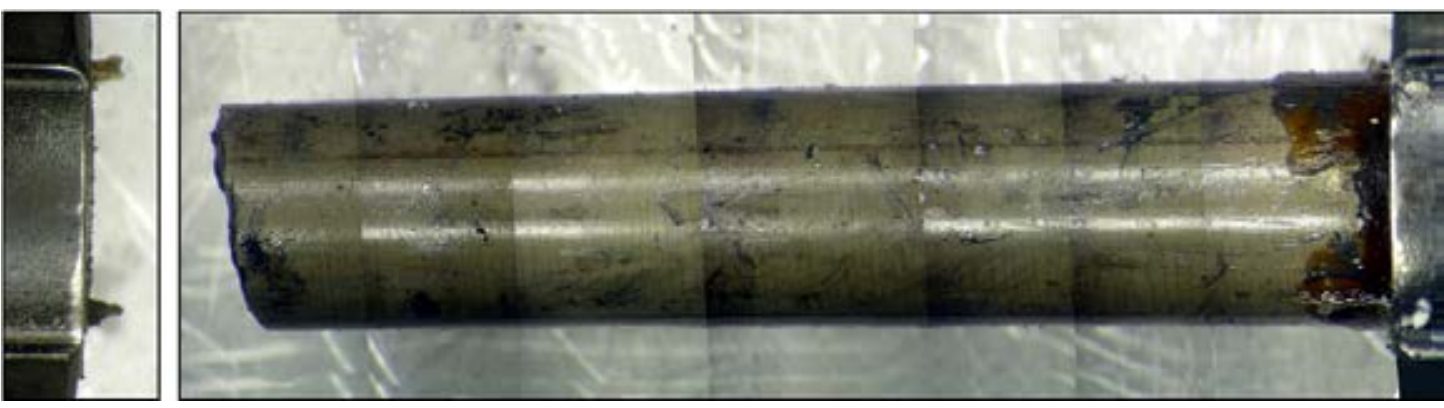

(c)
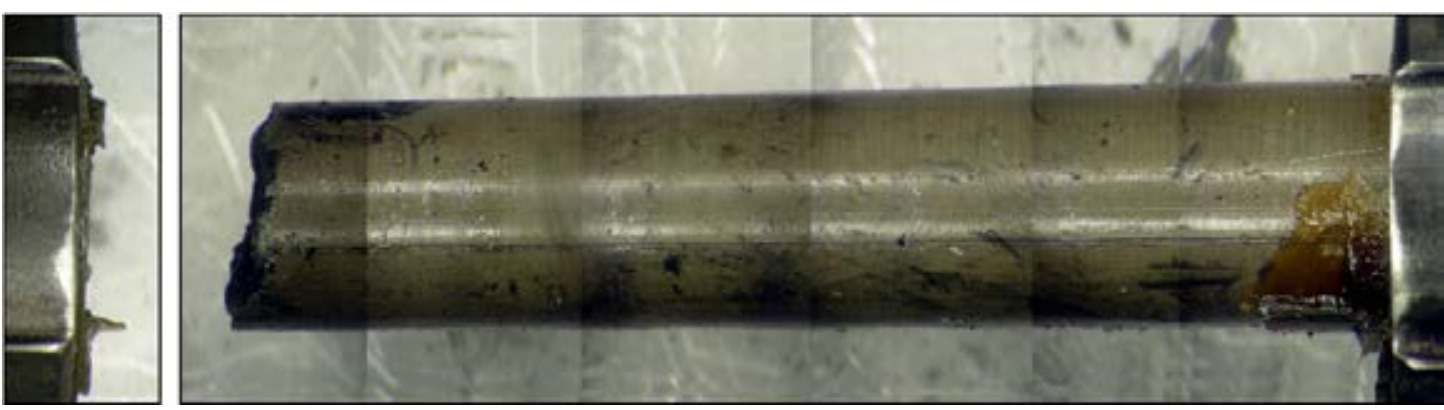

(d)

Figure E.19. (a, b) Mating fracture surfaces, (c) front side, and (d) back side of D6/ DH1 $(609 \mathrm{C} 4) ; \mathrm{N}_{\mathrm{f}}=2.4 \times 10^{5}$ cycles under $\pm 12.7 \mathrm{~N} \cdot \mathrm{m} 5 \mathrm{~Hz}$.

\section{D7/ DH2/ 609C3 ( $\pm 20.32 \mathrm{~N} \cdot \mathrm{M} 5 \mathrm{HZ})$}

The cycle test on D7/ DH2 (609C3, 66.5 GWd/MTU burnup, 70-100 $\mu \mathrm{m}$ oxide layer, 750 ppm H content) was conducted under $\pm 20.32 \mathrm{~N} \cdot \mathrm{m} 5 \mathrm{~Hz}$. DH2 fractured around $6.5 \times 10^{4}$ cycles.

On-line monitoring displayed a steady decrease throughout the cycle test with a sudden drop near the end (Figure E.20). The measurements using 0.8 and $1.2 \mathrm{~mm}$ also showed a similar rigidity variation as given in Figure E.21. 
$\mathrm{DH} 2$ fractured in the middle of the gage section on a pellet-pellet interface as can be seen from the clean cut in Figure E.22. Equally spaced circumferential cracks can be observed over both stressed surfaces of the rod with some spalling near the fracture.

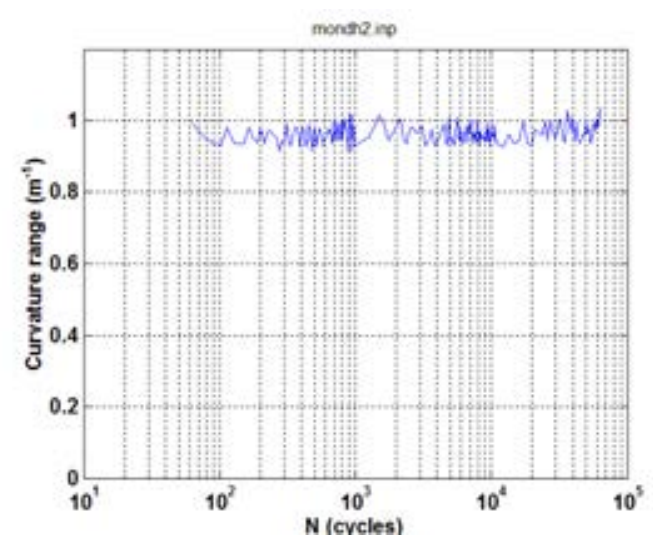

(a)

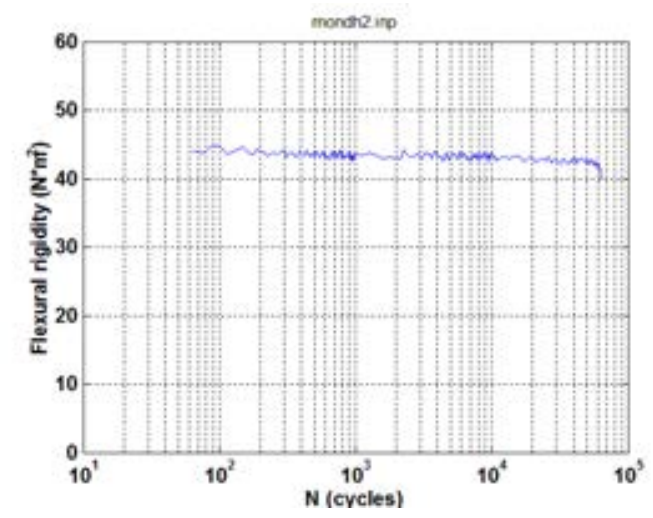

(c)

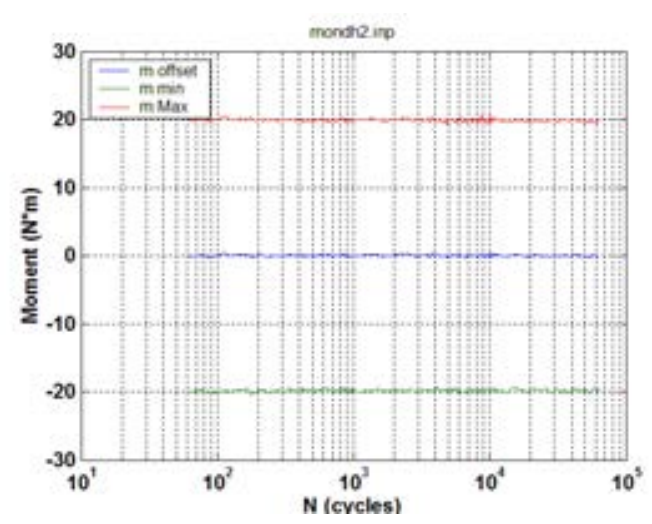

(e)

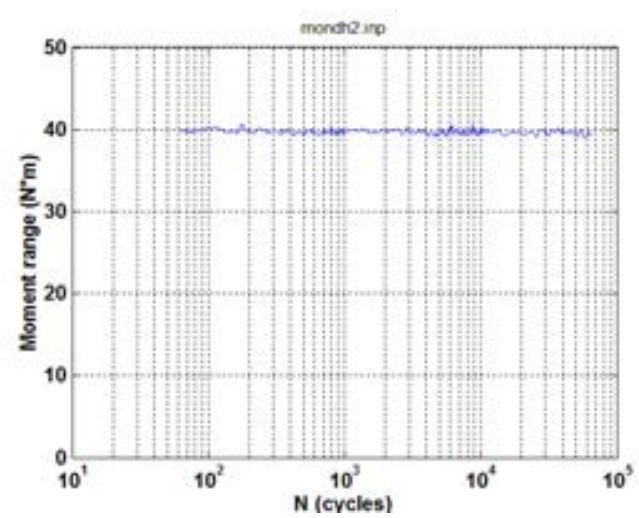

(b)

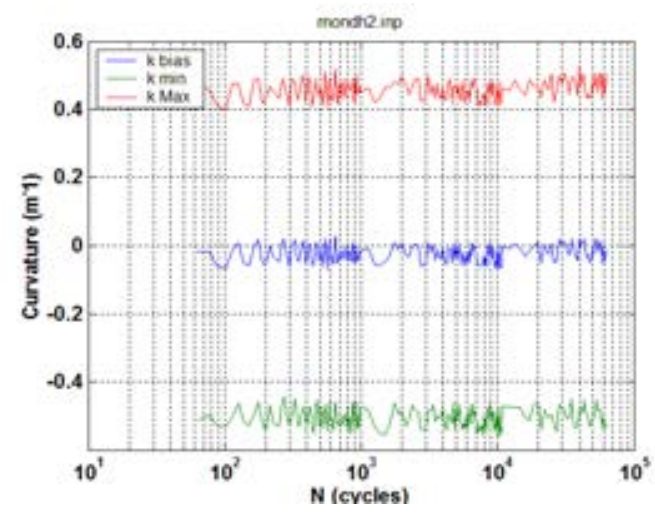

(d)

d)

Figure E.20. Variations of (a) curvature range, (b) applied moment range, (c) flexural rigidity, (d) maximum and minimum values of curvature, and (e) maximum and minimum values of moment as a function of number of cycles for D7I DH2 (609C3); $\mathrm{N}_{\mathrm{f}}=6.5 \times 10^{4}$ cycles under $\pm 20.32 \mathrm{~N} \cdot \mathrm{m} 5 \mathrm{~Hz}$. 


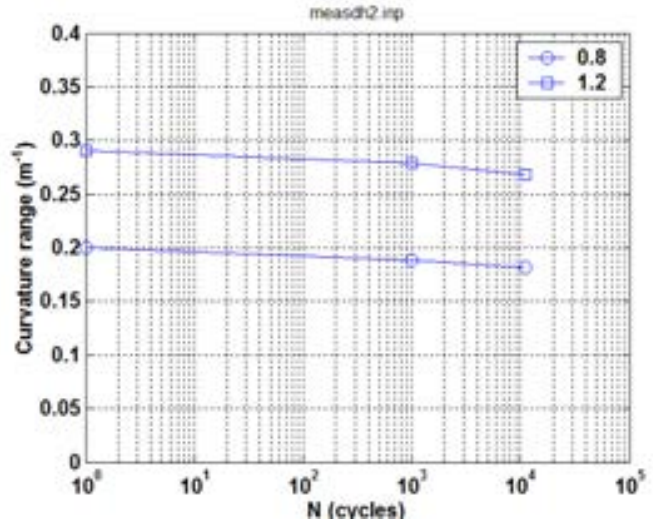

(a)

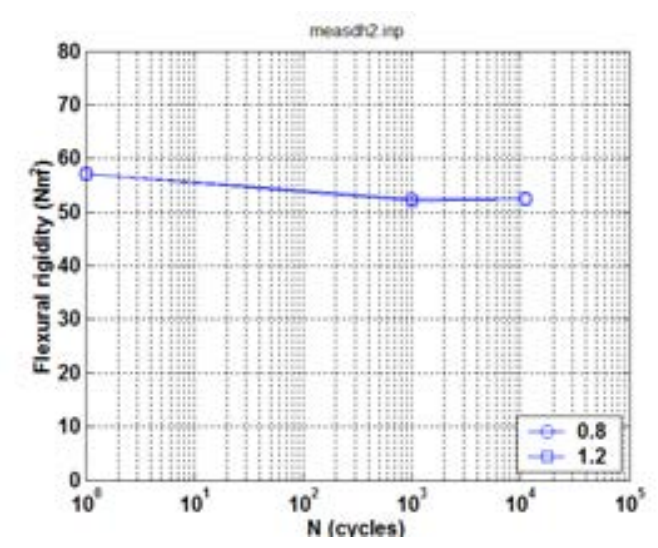

(c)

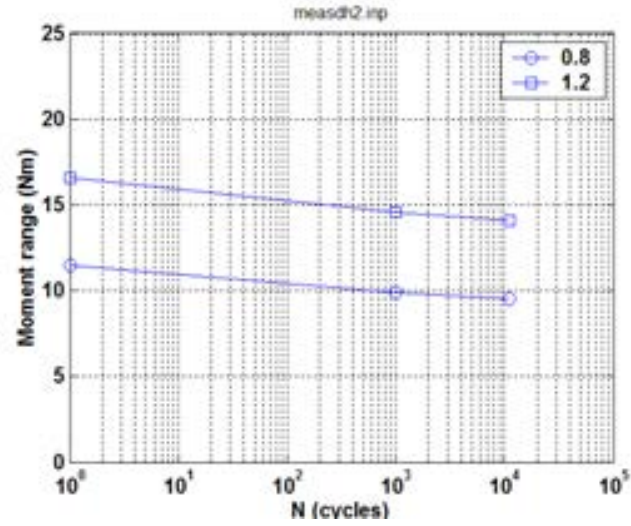

(b)

(b)

Figure E.21. Variations of (a) curvature range, (b) applied moment range, (c) flexural rigidity as a function of number of cycles for D7/ DH2 (609C3) based on measurements with maximum relative displacement at 0.8 and $1.2 \mathrm{~mm} ; \mathrm{N}_{\mathrm{f}}=6.5 \times 10^{4}$ cycles under \pm 20.32 $\mathrm{N} \cdot \mathrm{m} 5 \mathrm{~Hz}$. 


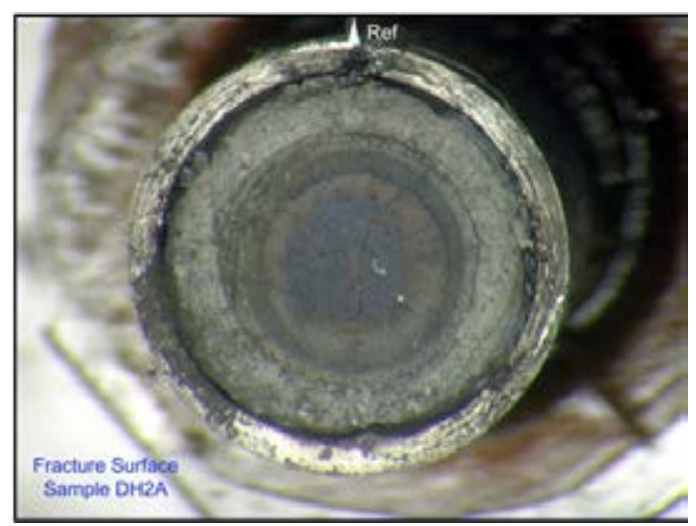

(a)

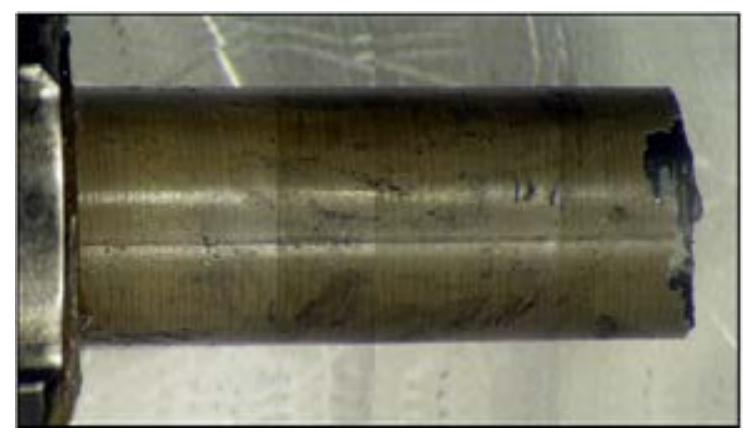

(c)
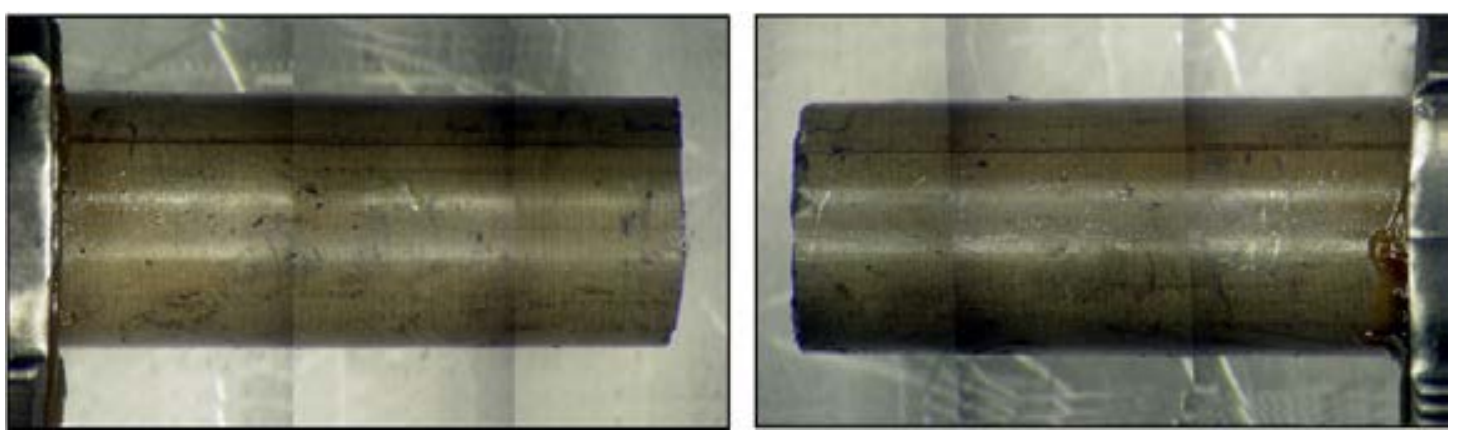

(d)

Figure E.22. (a, b) Mating fracture surfaces, (c) front side, and (d) back side of D7I DH2 $(609 C 3) ; N_{f}=6.5 \times 10^{4}$ cycles under $\pm 20.32 \mathrm{~N} \cdot \mathrm{m} 5 \mathrm{~Hz}$.

\section{D8/ DM3/ 606C3E ( \pm 8.89 N·M 5 HZ)}

D8/ DM3 (606C3E, 66.8 GWd/MTU burnup, 70-100 $\mu \mathrm{m}$ oxide layer, 550 ppm H content) sustained quite a long testing period under $\pm 8.89 \mathrm{~N} \cdot \mathrm{m} 5 \mathrm{~Hz}$. Testing was stopped after $1.3 \times 10^{7}$ cycles.

On-line monitoring displayed a stable flexural rigidity response, followed by a fluctuation or falland-rise near $4 \times 10^{6}$ cycles (Figure E.23). The fluctuation was limited within the range of 45 to $50 \mathrm{~N} \cdot \mathrm{m}^{2}$. The measurements using 0.8 and $1.2 \mathrm{~mm}$ also revealed a variation of a quite similar pattern in rigidity, as shown in Figure E.24. 


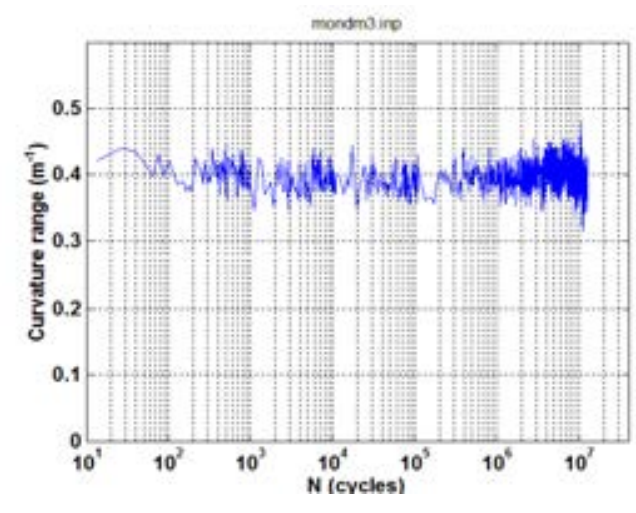

(a)

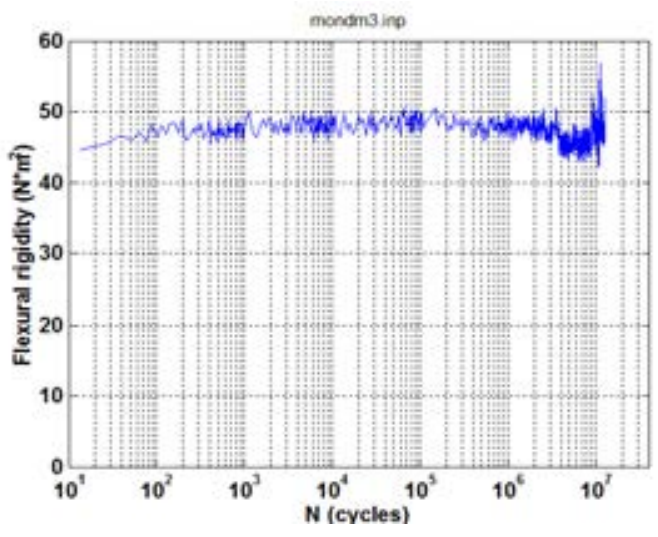

(c)

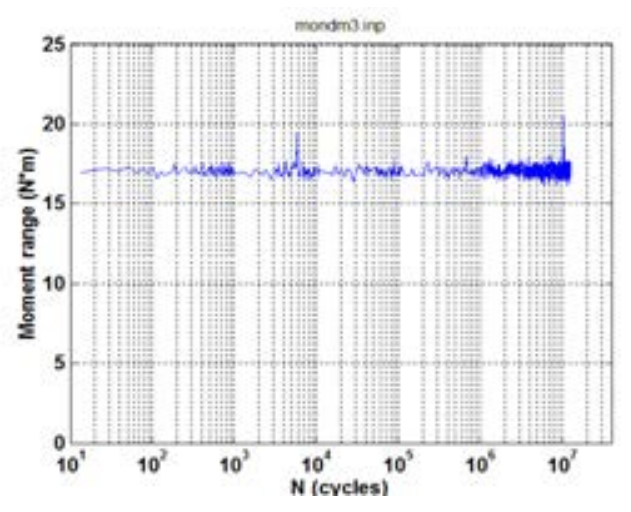

(b)

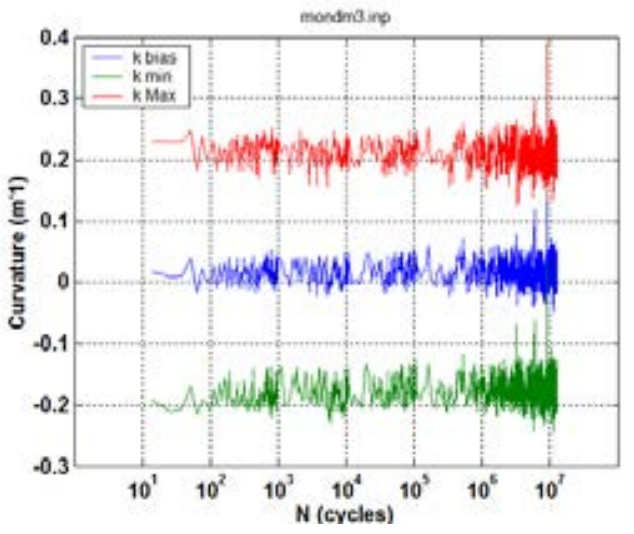

(d)

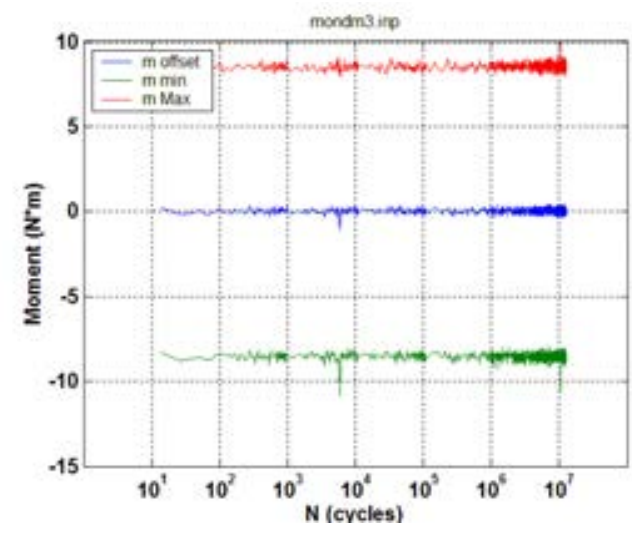

(e)

Figure E.23. Variations of (a) curvature range, (b) applied moment range, (c) flexural rigidity, (d) maximum and minimum values of curvature, and (e) maximum and minimum values of moment as a function of number of cycles for D8/ DM3 (606C3E); $\mathrm{N}=1.3 \times 10^{7}$ cycles under $\pm 8.89 \mathrm{~N} \cdot \mathrm{m} 5 \mathrm{~Hz}$. 


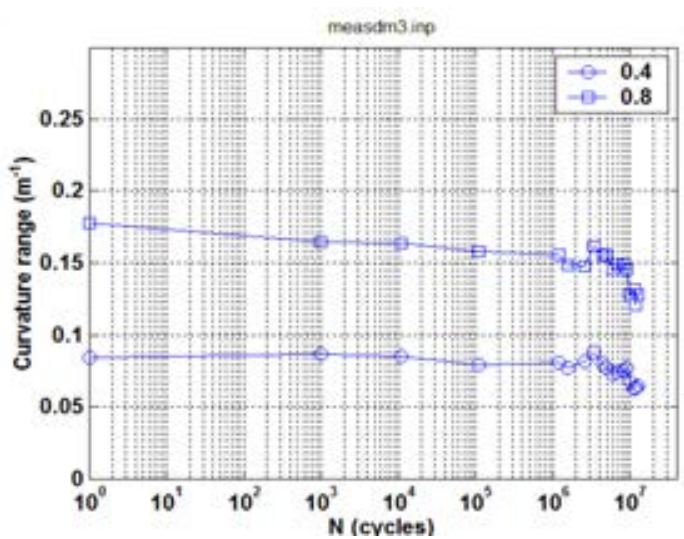

(a)

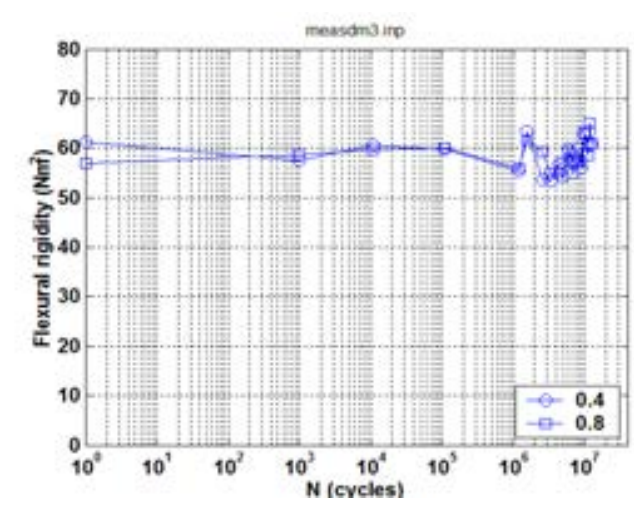

(c)

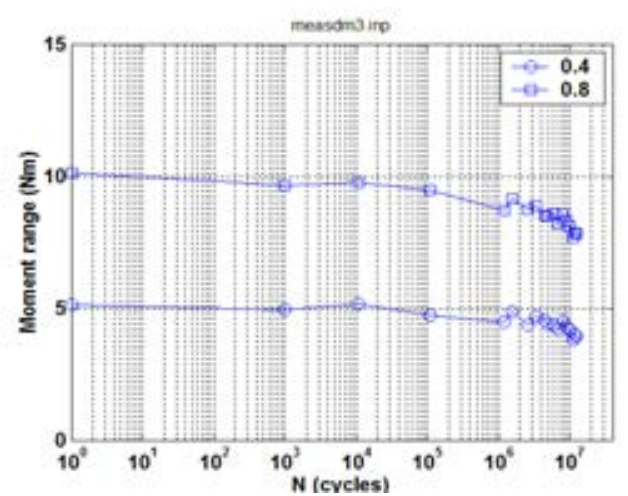

(b)

\section{(b)}

(b)




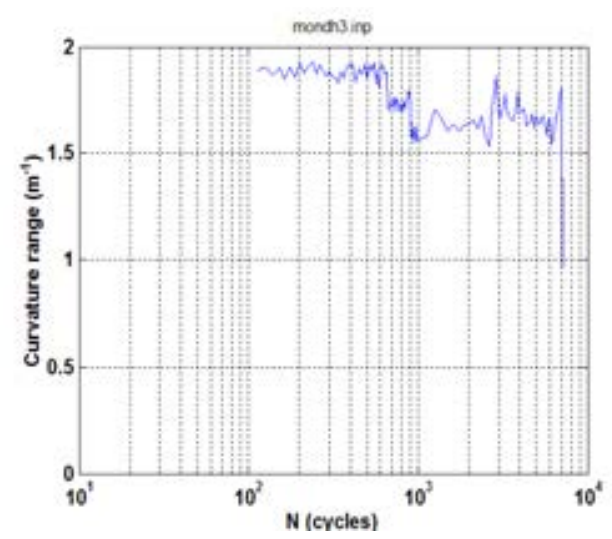

(a)

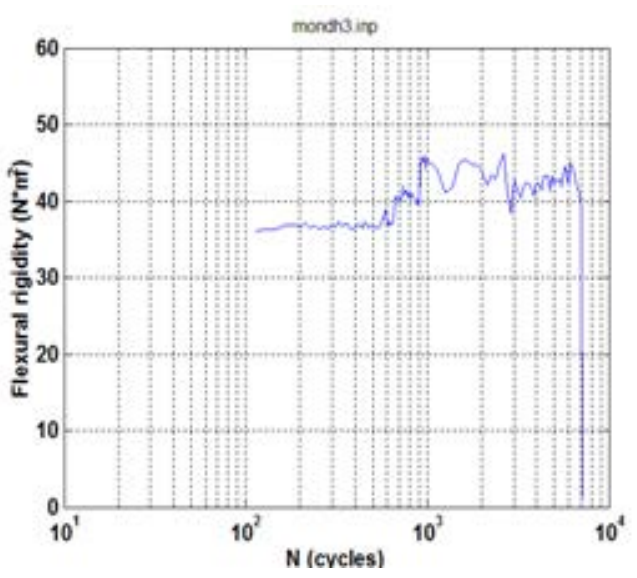

(c)

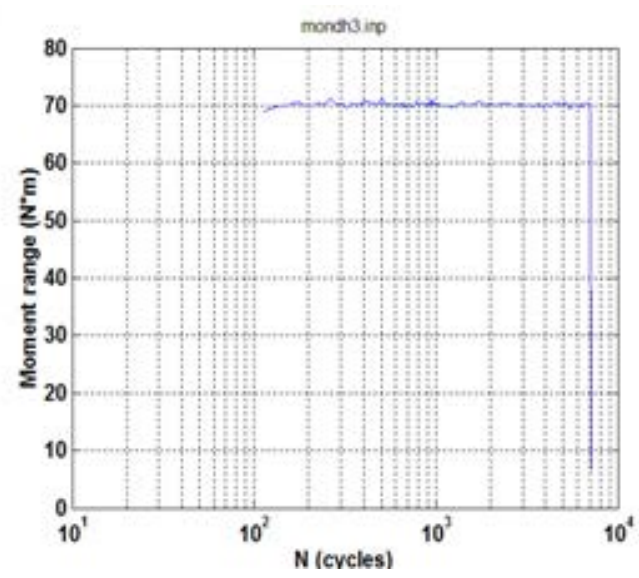

(b)

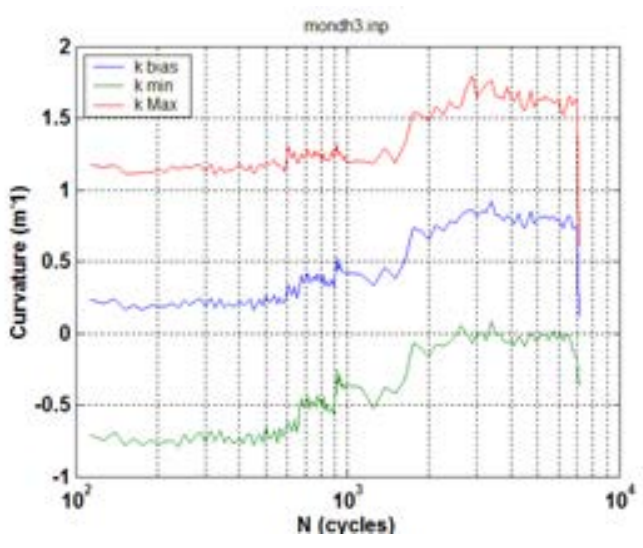

(d)

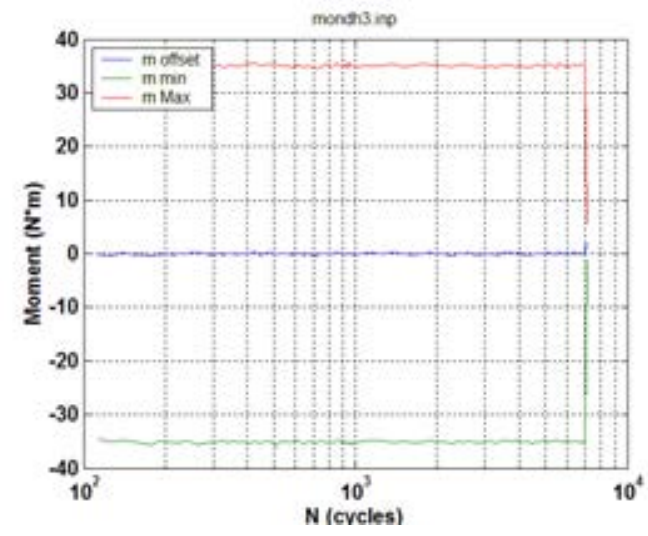

(e)

Figure E.25. Variations of (a) curvature range, (b) applied moment range, (c) flexural rigidity, (d) maximum and minimum values of curvature, and (e) maximum and minimum values of as a function of number of cycles for D9/ DH3 (609C7); $N_{f}=7.13 \times 10^{3}$ cycles under $\pm 35.56 \mathrm{~N} \cdot \mathrm{m} 5 \mathrm{~Hz}$. 


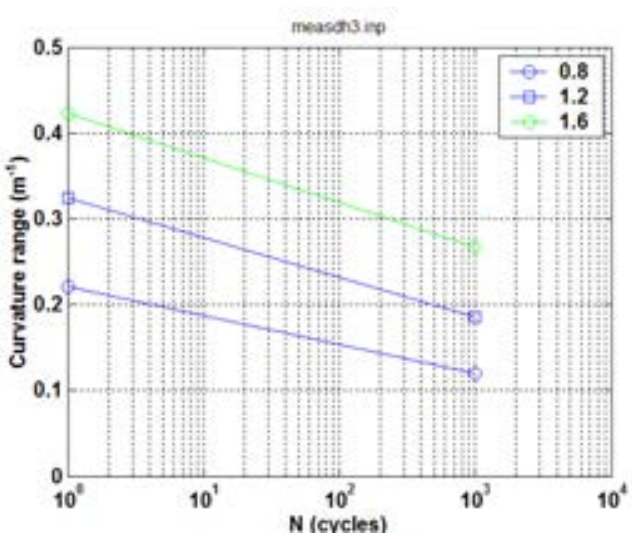

(a)

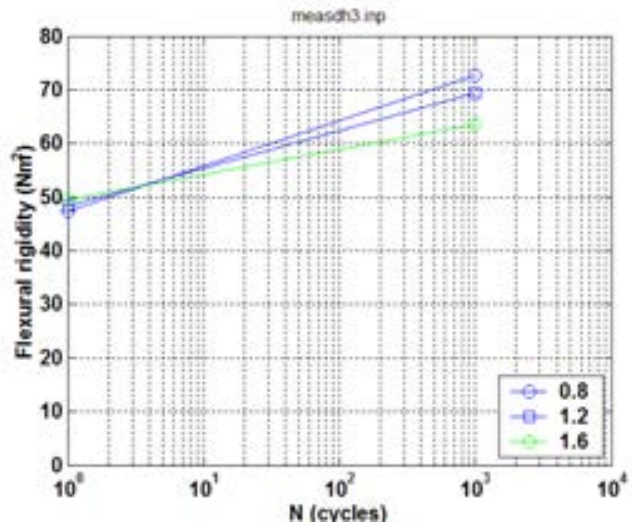

(c)

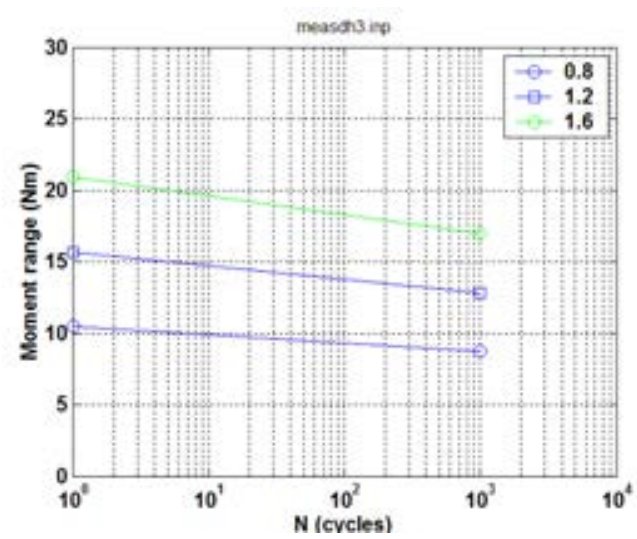

(b)

Figure E.26. Variations of (a) curvature range, (b) applied moment range, and (c) flexural rigidity as a function of number of cycles for D9/ DH3 $(609 \mathrm{C}) ; \mathrm{N}_{\mathrm{f}}=7.13 \times 10^{3}$ cycles under $\pm 35.56 \mathrm{~N} \cdot \mathrm{m} 5 \mathrm{~Hz}$.

\section{D10/ S5/ 606C3A ( $\pm 12.70 \mathrm{~N} \cdot \mathrm{M} 5 \mathrm{HZ})$}

The cycle test on D10/ S5 (606C3A, 66.8 GWd/MTU burnup, 70-100 $\mu \mathrm{m}$ oxide layer, 750 ppm $\mathrm{H}$ content) was conducted under $\pm 12.70 \mathrm{~N} \cdot \mathrm{m} 5 \mathrm{~Hz}$. The testing condition was the same as that used in $\mathrm{DH} 1$, where failure took place near the edge of the rigid sleeve. The test was repeated to confirm the lifetime and failure position under the same testing condition. S5, in fact, failed around $1.81 \times 10^{5}$ cycles within the gage section. The lifetime was thus a little shorter than that $\mathrm{DH} 1$. The difference may be related to the preexisting condition as a little higher estimated $\mathrm{H}$ content in $\mathrm{S} 5$ was seen.

The equivalent decrease in flexural rigidity prior to final failure in both monitoring and measurement can be seen clearly from Figures E.27 and E.28, respectively. Detailed examination showed a certain degree of curvature waveform drift toward the positive direction. The positively biased curvature wave corresponded to an absolute extreme value as great as $0.4 \mathrm{~m}^{-1}$. The level of extreme curvature in D10/ S5 was clearly higher than that observed in $\mathrm{D} 6 / \mathrm{DH} 1$, which was also partly responsive to the shorter lifetime in the former case. 


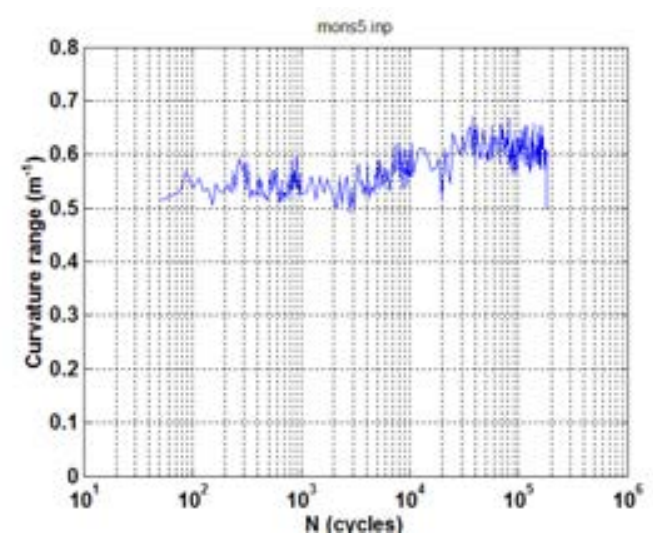

(a)

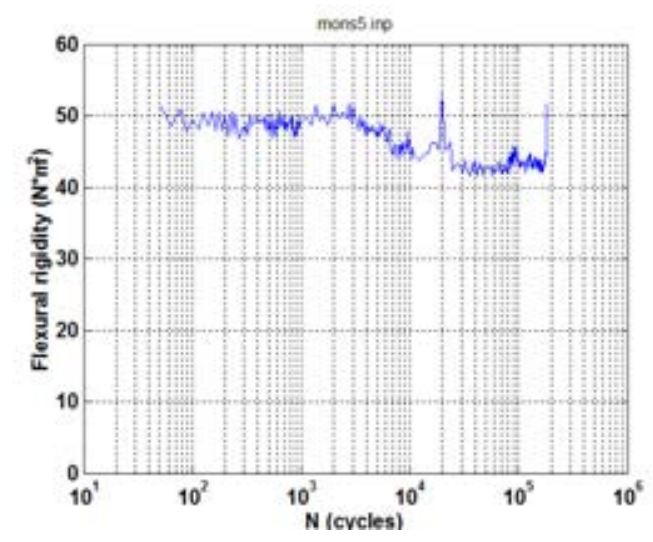

(c)

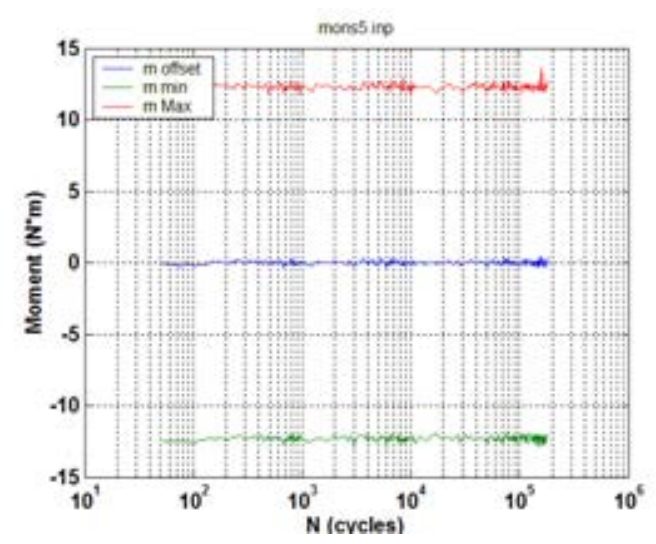

(e)

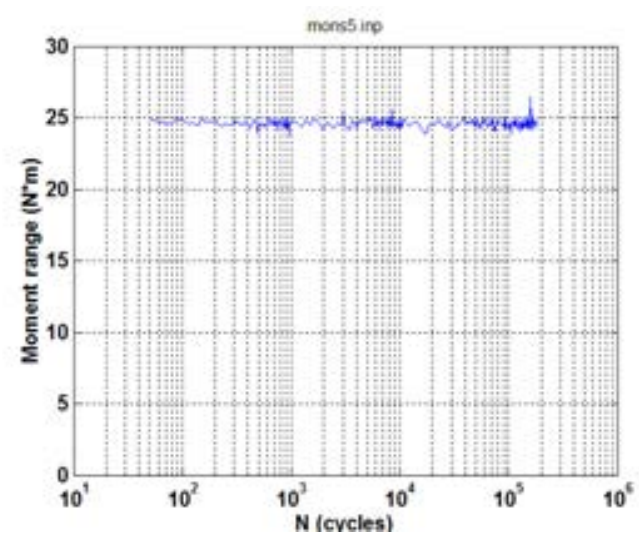

(b)

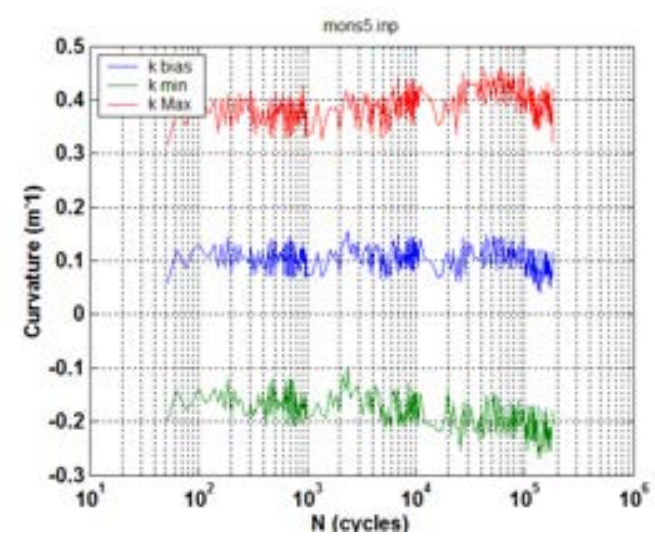

(d)

Figure E.27. Variations of (a) curvature range, (b) applied moment range, (c) flexural rigidity, (d) maximum and minimum values of curvature, and (e) maximum and minimum values of moment as a function of number of cycles for D10/ S5 $(606 \mathrm{C} 3 \mathrm{~A}) ; \mathrm{N}_{\mathrm{f}}=1.81 \times 10^{5}$ cycles under $\pm 12.70 \mathrm{~N} \cdot \mathrm{m} 5 \mathrm{~Hz}$. 


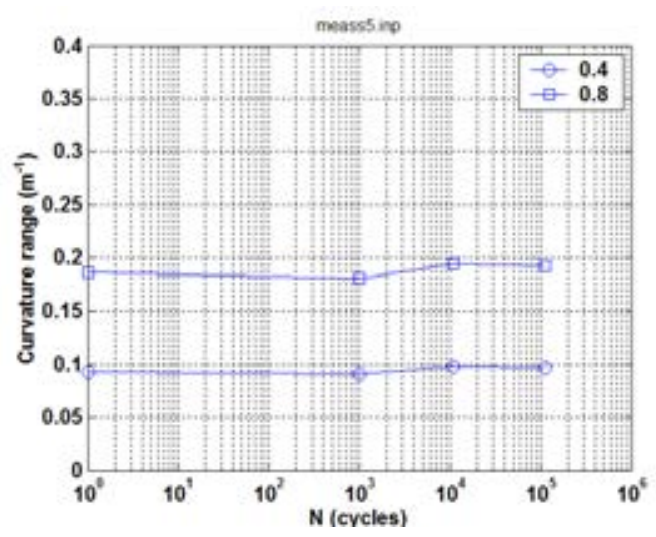

(a)

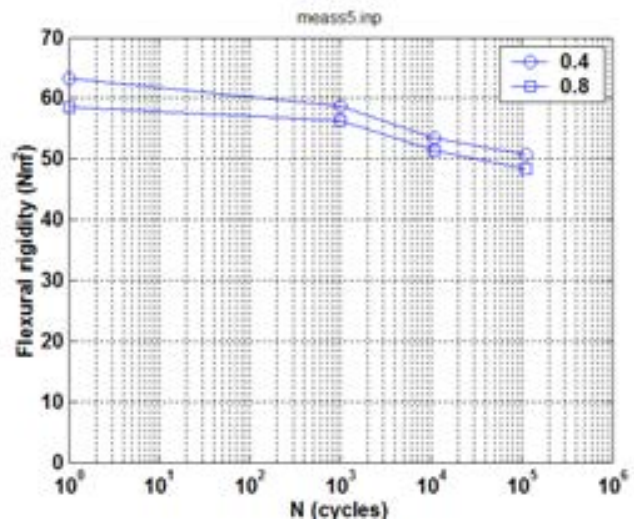

(c)

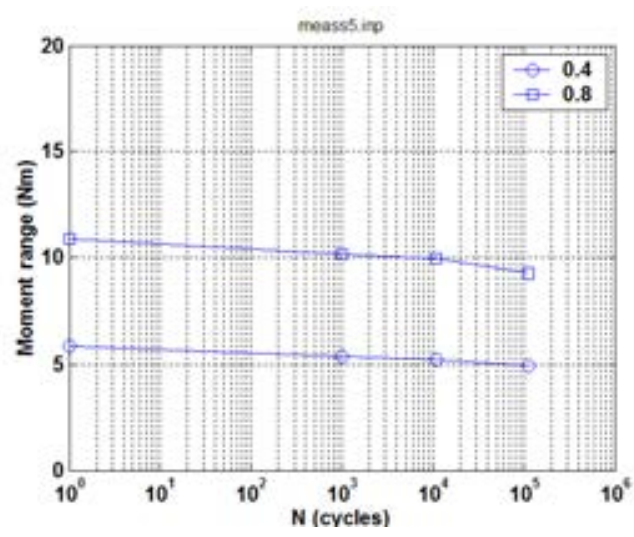

(b)

Figure E.28. Variations of (a) curvature range, (b) applied moment range, and (c) flexural rigidity as a function of number of cycles for D10/ S5 $(606 \mathrm{C} 3 \mathrm{~A}) ; \mathrm{N}_{\mathrm{f}}=1.81 \times 10^{5}$ cycles under $\pm 12.70 \mathrm{~N} \cdot \mathrm{m} 5 \mathrm{~Hz}$.

\section{D11/ R1/ 607C4A ( $\pm 30.48 \mathrm{~N} \cdot \mathrm{M} 5 \mathrm{HZ})$}

The cycle test on D11/ R1 (606C3A, $63.8 \mathrm{GWd/MTU}$ burnup, 70-100 $\mu \mathrm{m}$ oxide layer, 700 ppm $\mathrm{H}$ content) was conducted under $\pm 30.48 \mathrm{~N} \cdot \mathrm{m} 5 \mathrm{~Hz}$. R1 failed around $5.5 \times 10^{3}$ cycles within the gage section. Significant fall and rise were observed in on-line monitoring between $1 \times 10^{3}$ and $4 \times 10^{3}$ cycles as shown in Figure E.29. On the other hand, the measurements using 0.8, 1.2, and $1.6 \mathrm{~mm}$ relative displacement at loading points demonstrated a defined decrease in flexural rigidity as seen in Figure E.30. Such lifetime was obviously shorter than that expected from the result of $\mathrm{D} 9 / \mathrm{DH} 3$, where a longer lifetime was obtained with an even higher moment level. Considering that the $\mathrm{H}$ content of $\mathrm{D} 9 / \mathrm{DH} 3,550 \mathrm{ppm}$, was much lower than that of D11/ R1, it is believed that the preexisting condition of specimens played a certain role in controlling failure in high burnup, irradiated rods as can be seen from the estimated $\mathrm{H}$ contents in both rod segments. 


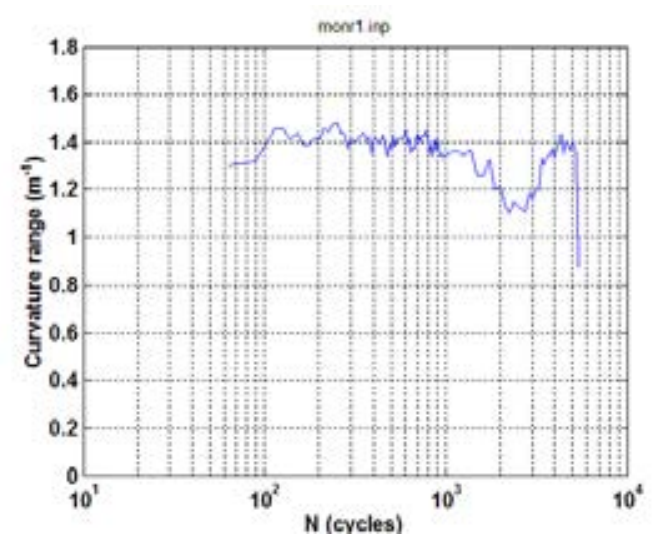

(a)

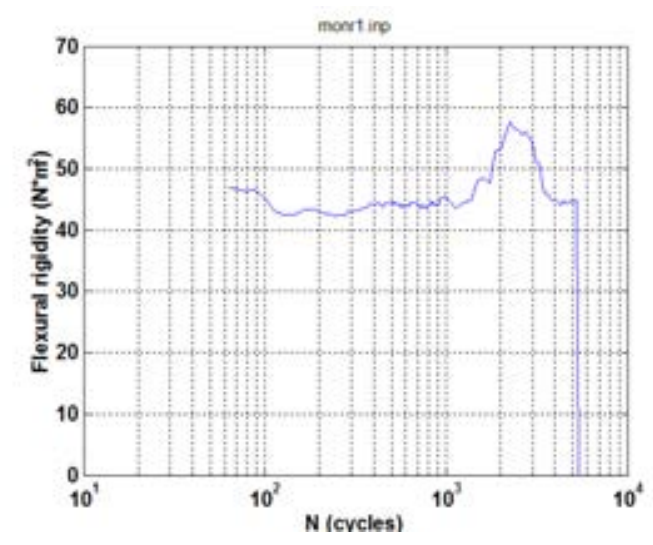

(c)

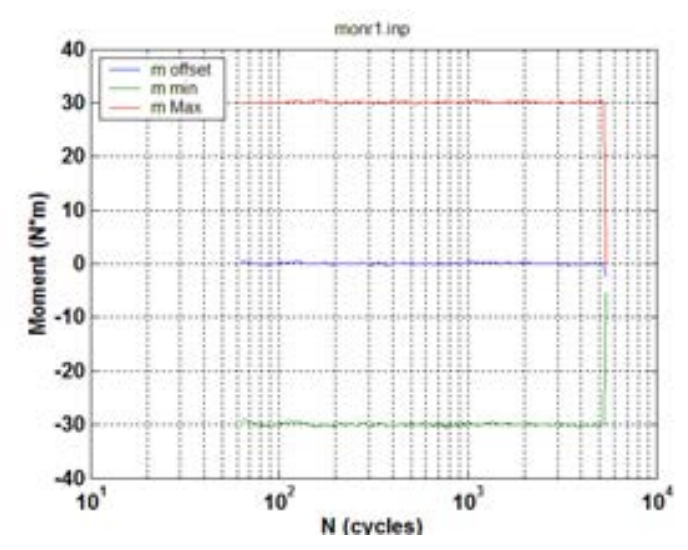

(e)

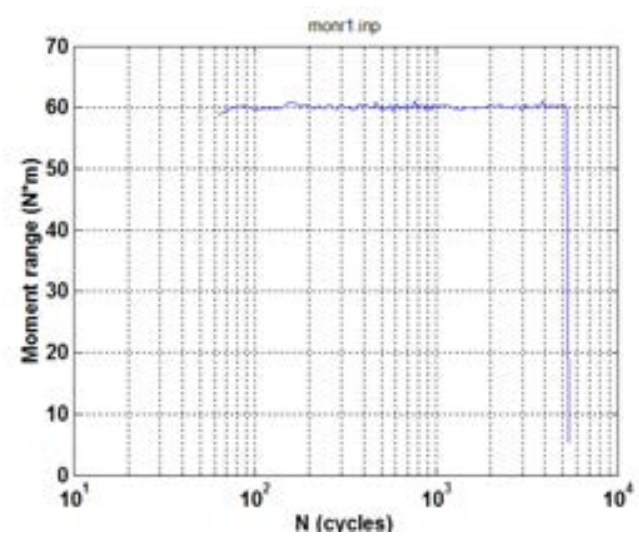

(b)

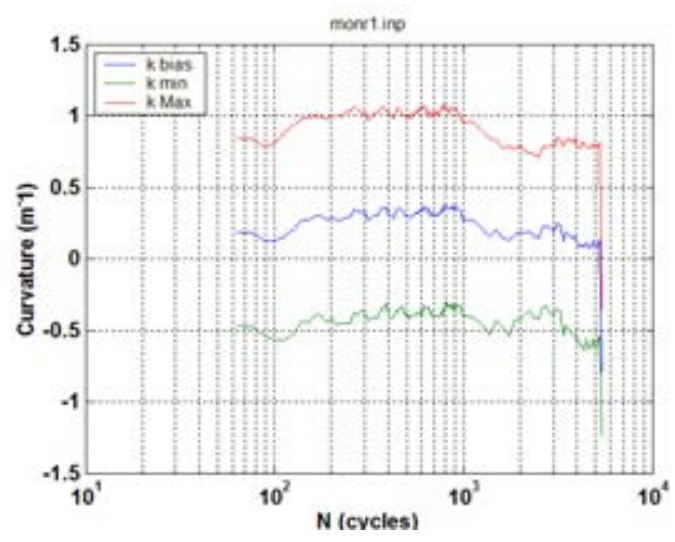

(d)

Figure E.29. Variations of (a) curvature range, (b) applied moment range, (c) flexural rigidity, (d) maximum and minimum values of curvature, and (e) maximum and minimum values of moment as a function of number of cycles for D11/ R1 (607C4A); $N_{f}=5.5 \times 10^{3}$ cycles under $\pm 30.48 \mathrm{~N} \cdot \mathrm{m} 5 \mathrm{~Hz}$. 


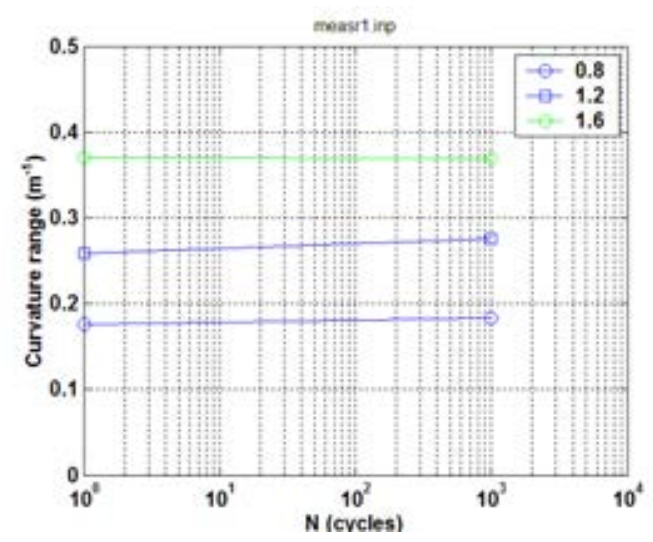

(a)

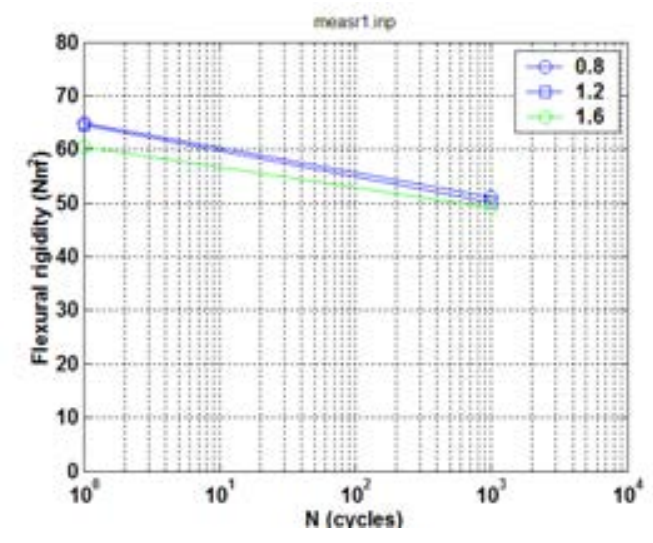

(c)

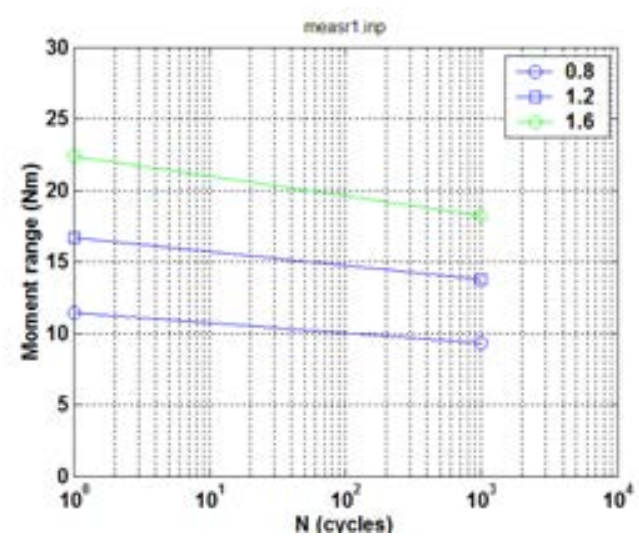

(b)

Figure E.30. Variations of (a) curvature range, (b) applied moment range, and (c) flexural rigidity as a function of number of cycles for D11/ R1 (607C4A); $N_{f}=5.5 \times 10^{3}$ cycles under $\pm 30.48 \mathrm{~N} \cdot \mathrm{m} 5 \mathrm{~Hz}$.

\section{D12/ R2/ 608C4A ( $\pm 11.18 \mathrm{~N} \cdot \mathrm{M} 5 \mathrm{HZ})$}

The cycle test on D12/ R2 (608C4A, $63.8 \mathrm{GWd/MTU}$ burnup, 70-100 $\mu \mathrm{m}$ oxide layer, 700 ppm $\mathrm{H}$ content) was conducted under $\pm 11.18 \mathrm{~N} \cdot \mathrm{m} 5 \mathrm{~Hz}$. R2 failed around $3.86 \times 10^{5}$ cycles within the gage section.

A certain amount of fluctuation was observed in on-line monitoring and measurement data sets as illustrated in Figures E.31 and E.32, respectively. This can be seen around 104 cycles. The peak/valley monitoring indicated that the curvature waveform down shifted a little throughout the cycle test process. An abrupt drop in flexural rigidity was captured prior to the final failure in both monitoring and measurement. 


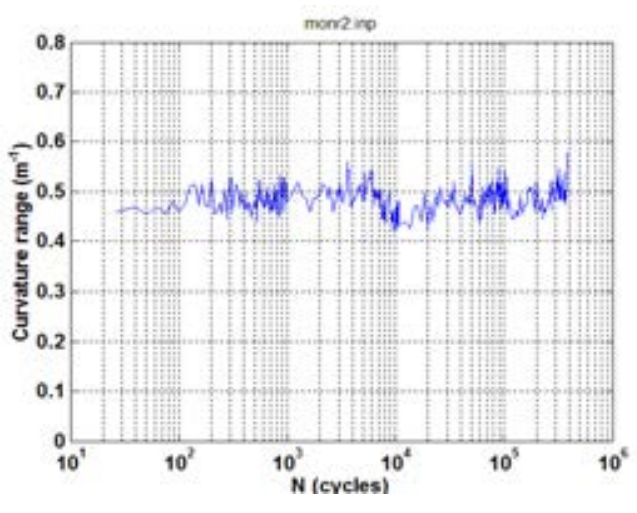

(a)

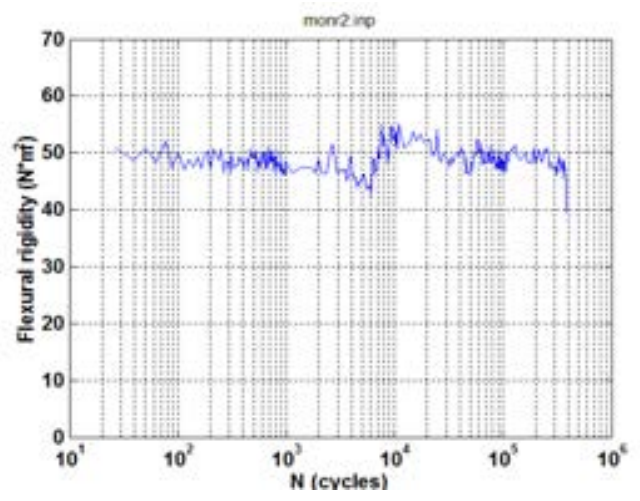

(c)

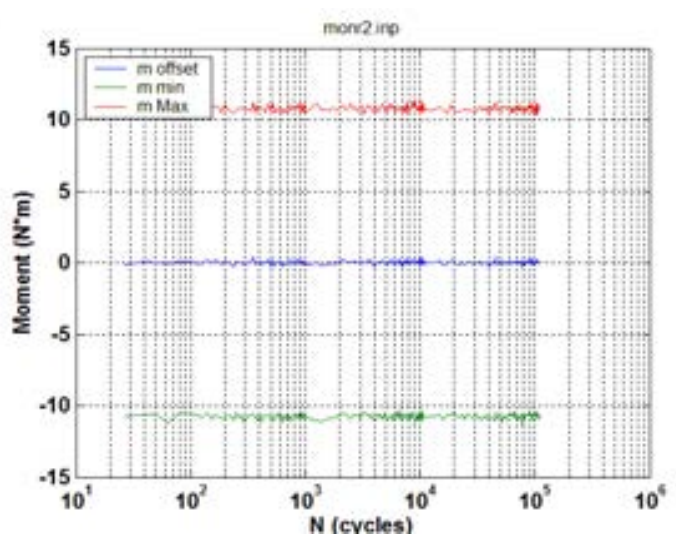

(e)

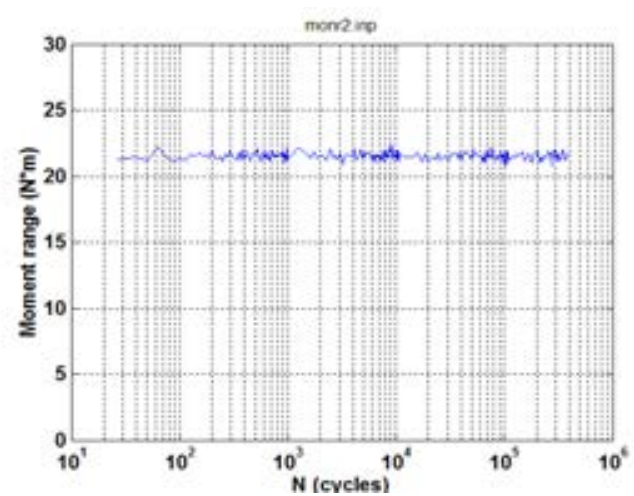

(b)

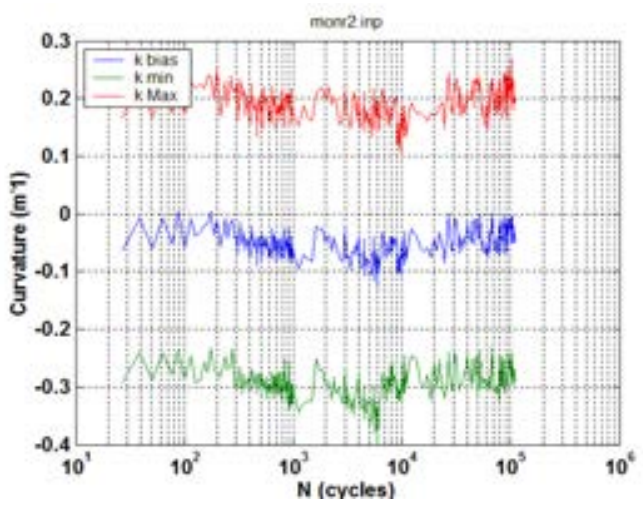

(d) 


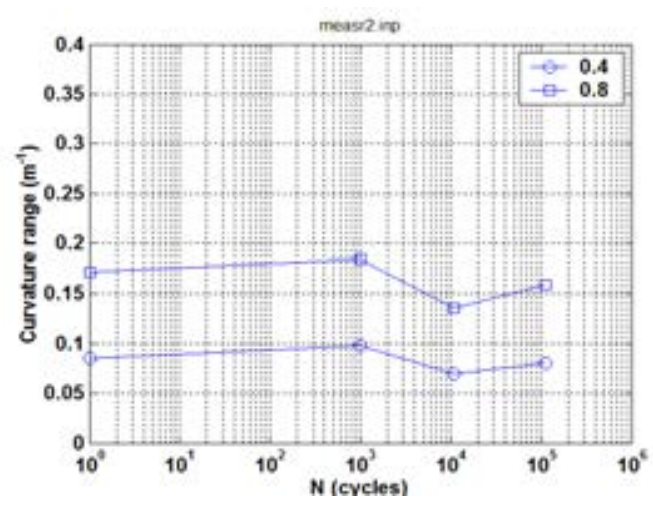

(a)

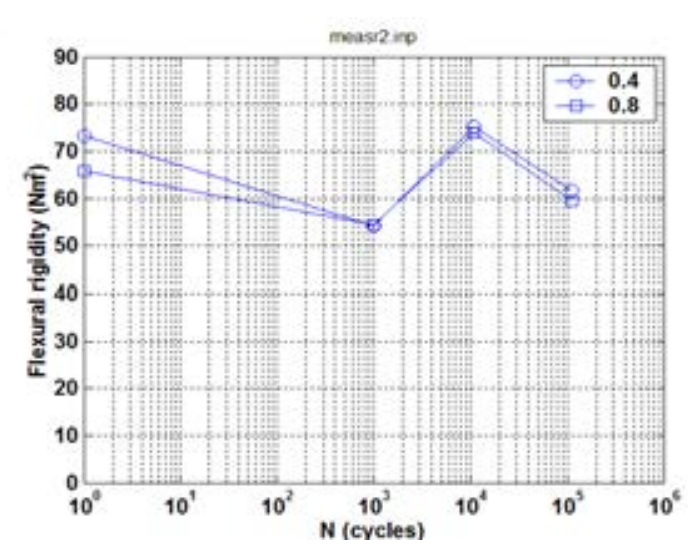

(c)

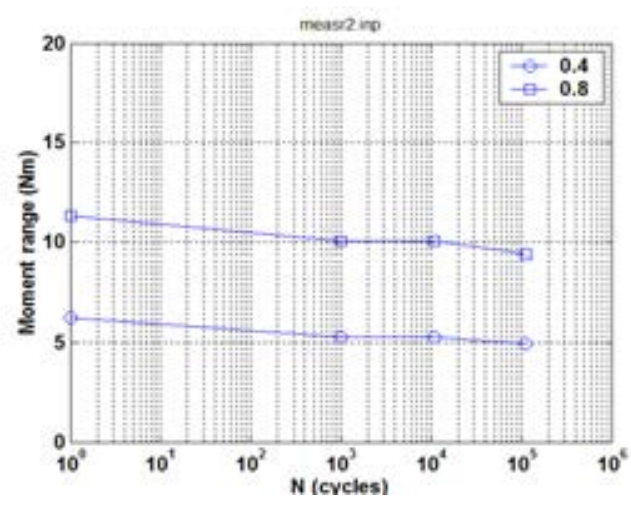

(b)

Figure E.32. Variations of (a) curvature range, (b) applied moment range, and (c) flexural rigidity as a function of number of cycles for D12/ R2 $(608 C 4 \mathrm{~A}) ; \mathrm{N}_{\mathrm{f}}=3.86 \times 10^{5}$ cycles under $\pm 11.18 \mathrm{~N} \cdot \mathrm{m} 5 \mathrm{~Hz}$.

\section{D13/ R3/ 606B3E ( $\pm 13.72 \mathrm{~N} \cdot \mathrm{M} 5 \mathrm{HZ})$}

The cycle test on D13/ R3 (606B3E, 66.5 GWd/MTU burnup, 100-110 $\mu$ m oxide layer, 750 ppm $\mathrm{H}$ content) was conducted under $\pm 13.72 \mathrm{~N} \cdot \mathrm{m} 5 \mathrm{~Hz}$. R3 failed around $1.29 \times 105$ cycles within the gage section.

Testing revealed that the on-line monitoring rigidity sustained a certain increase prior to the final drop at failure because of the continuous decrease in curvature range as shown in Figure E.33. The monitoring of the peak and valley of curvature indicated that the decreased curvature range is mainly due to the drop in peak value.

The cause of such single-side change in curvature waveform during the cyclic test is unknown, but decreased rigidity was indeed seen in the measurement shown in Figure E.34. 


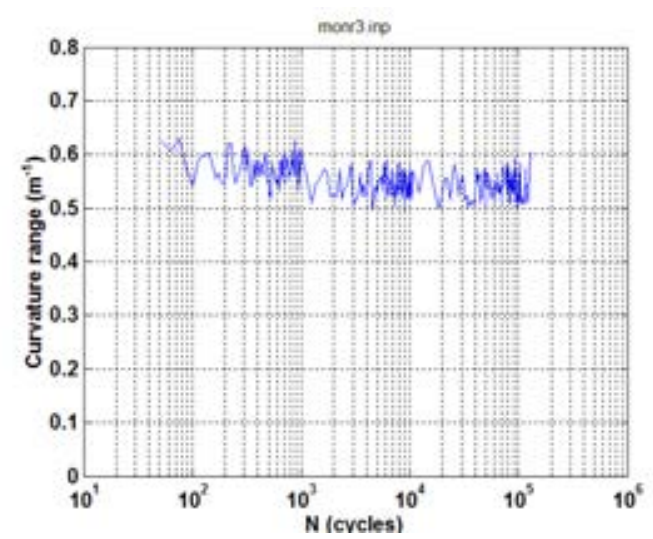

(a)

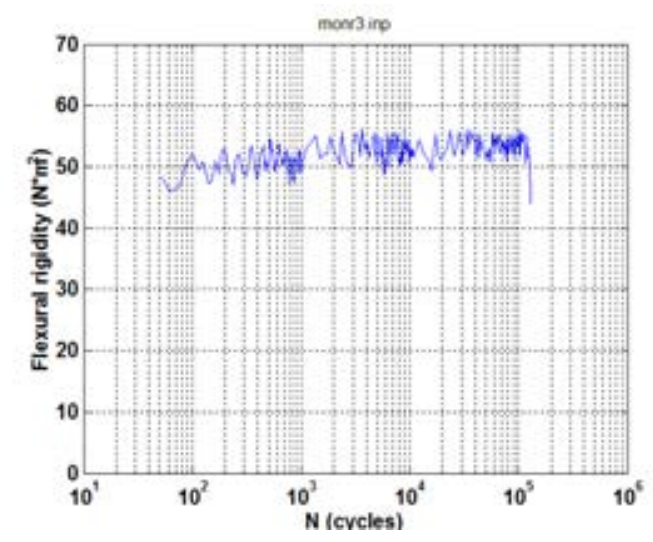

(c)

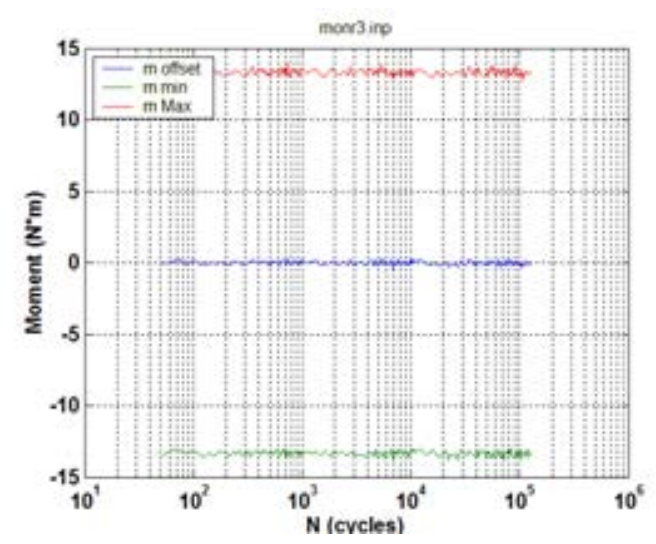

(e)

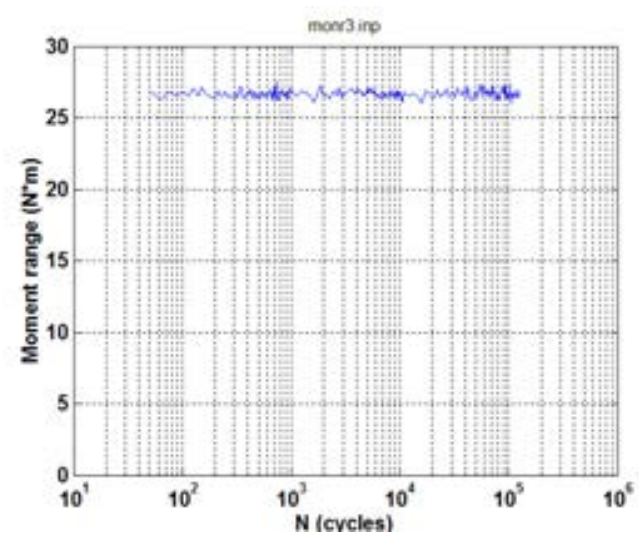

(b)

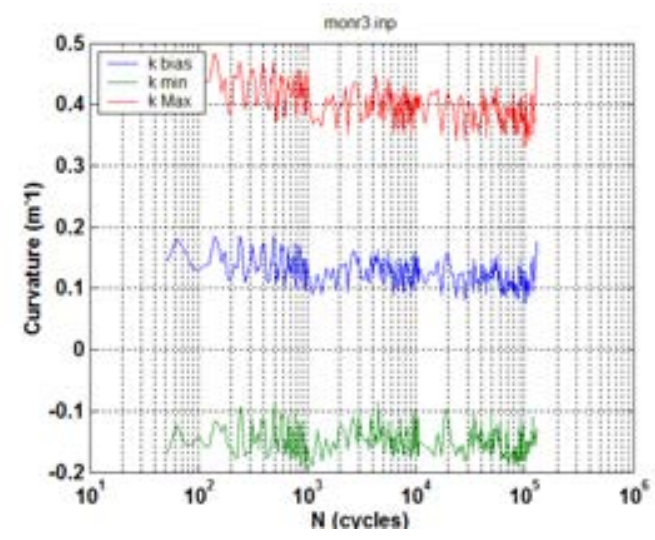

(d)

Figure E.33. Variations of (a) curvature range, (b) applied moment range, (c) flexural rigidity, (d) maximum and minimum values of curvature, and (e) maximum and minimum values of moment as a function of number of cycles for D13/ R3 (606B3E); $N_{f}=1.29 \times 10^{5}$ cycles under $\pm 13.72 \mathrm{~N} \cdot \mathrm{m} 5 \mathrm{~Hz}$. 


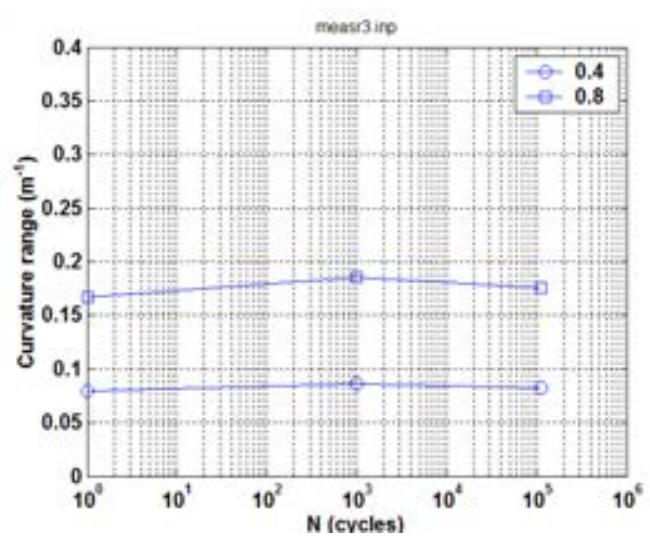

(a)

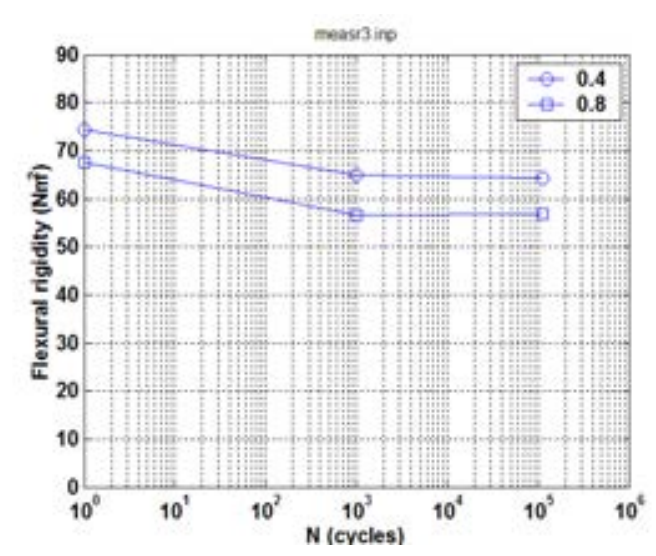

(c)

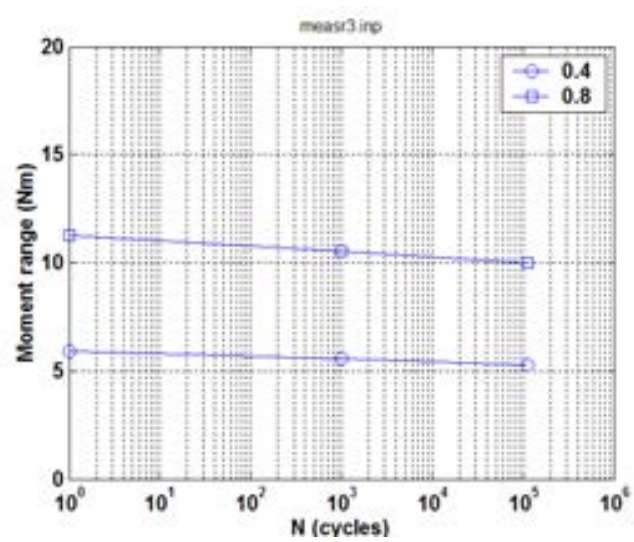

(b) 


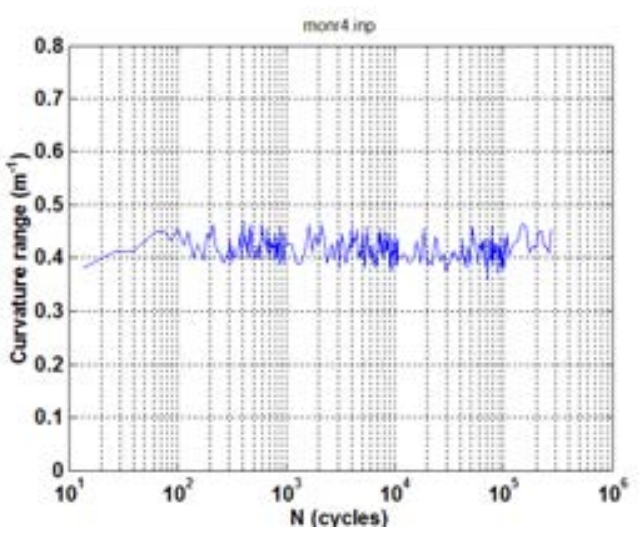

(a)

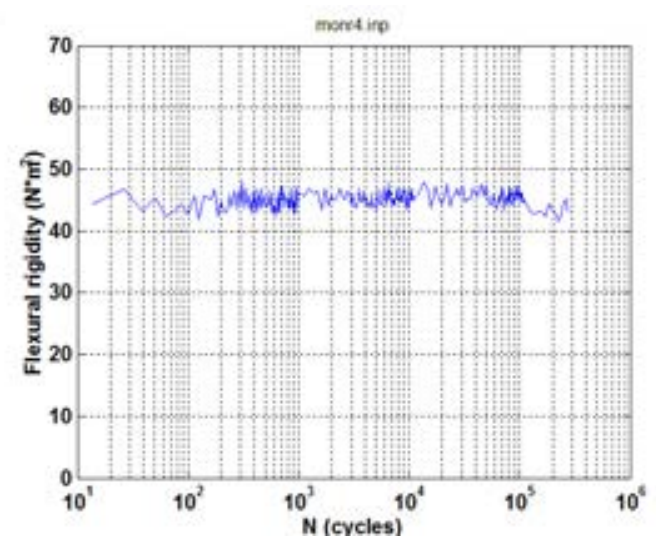

(c)

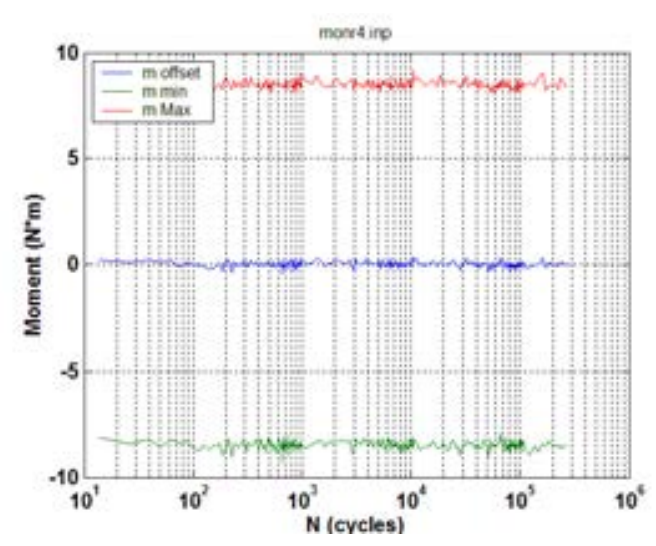

(e)

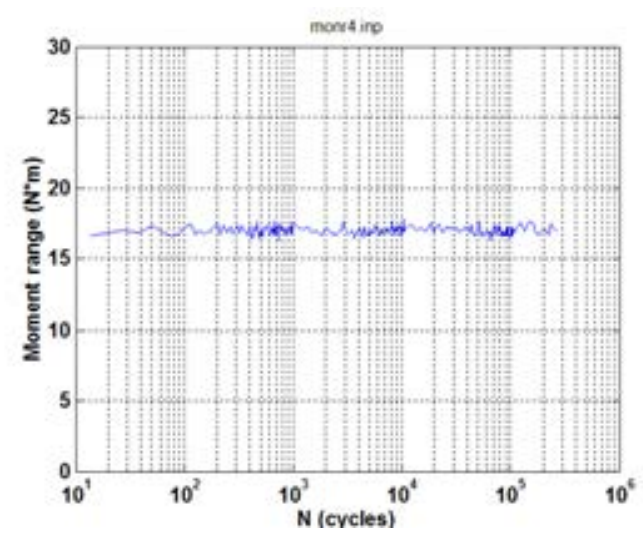

(b)

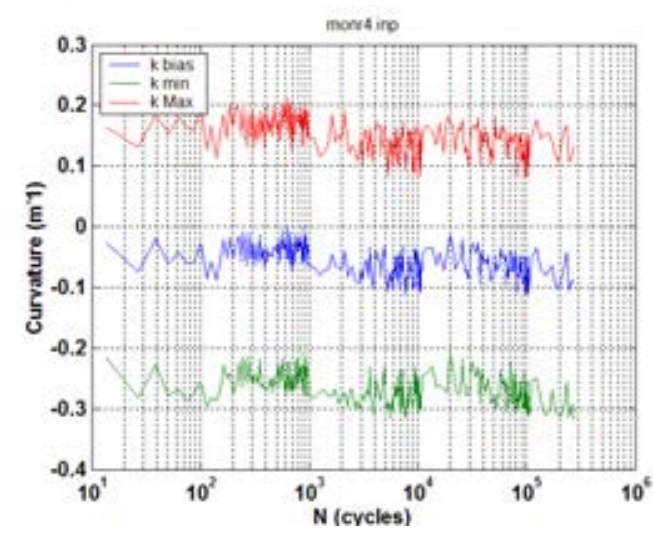

(d)

(d)

Figure E.35. Variations of (a) curvature range, (b) applied moment range, (c) flexural rigidity, (d) maximum and minimum values of curvature, and (e) maximum and minimum values of moment as a function of number of cycles for R4 (606B3D); $N_{f}=2.7 \times 10^{5}$ cycles under $\pm 8.89 \mathrm{~N} \cdot \mathrm{m} 5 \mathrm{~Hz}$. 


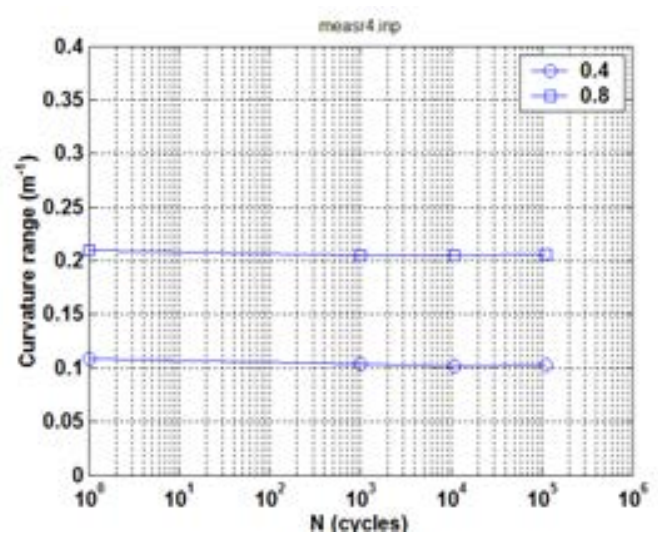

(a)

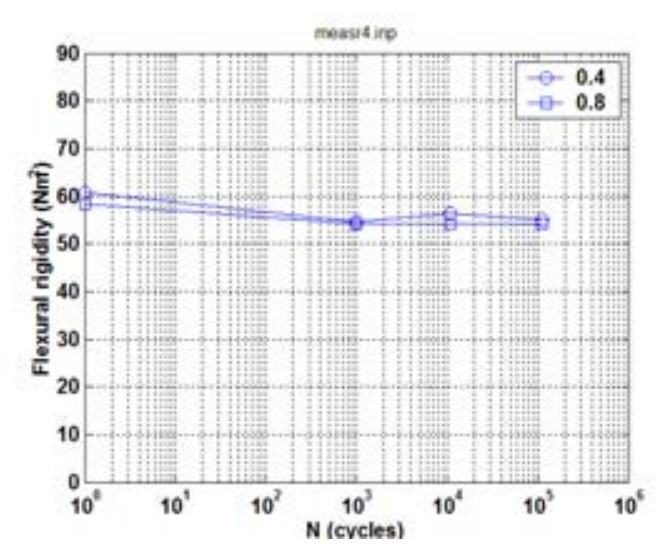

(c)

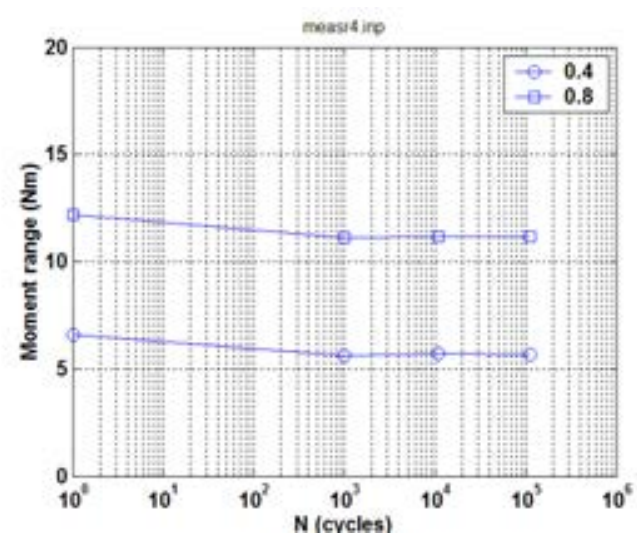

(b)

b) 


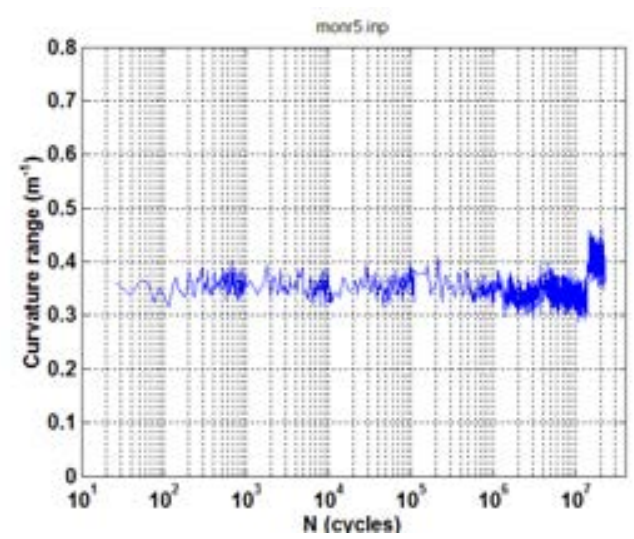

(a)

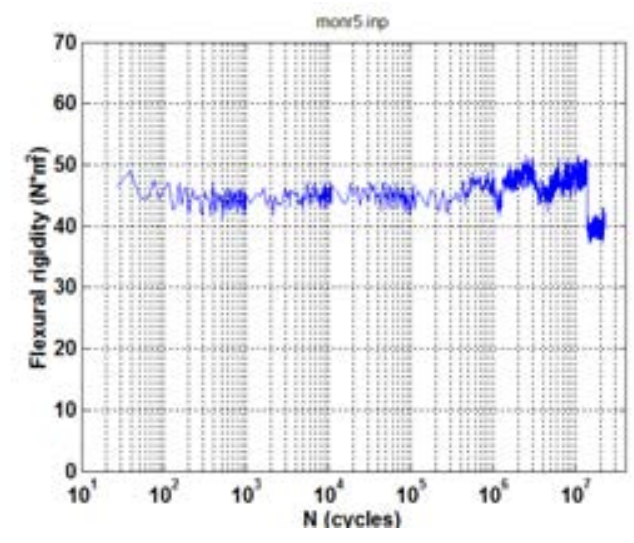

(c)

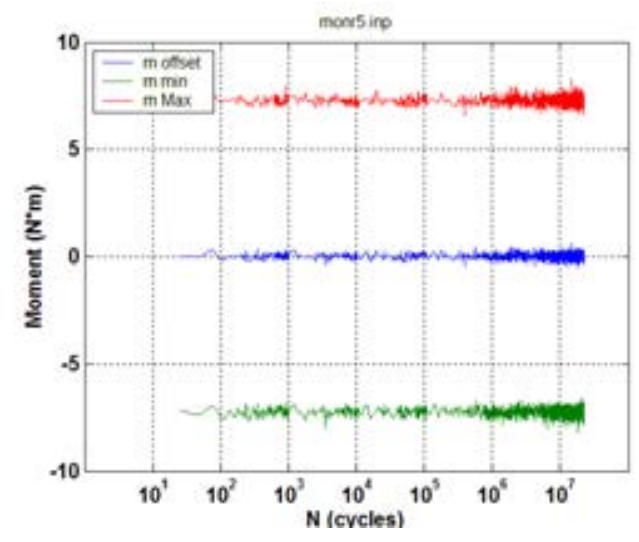

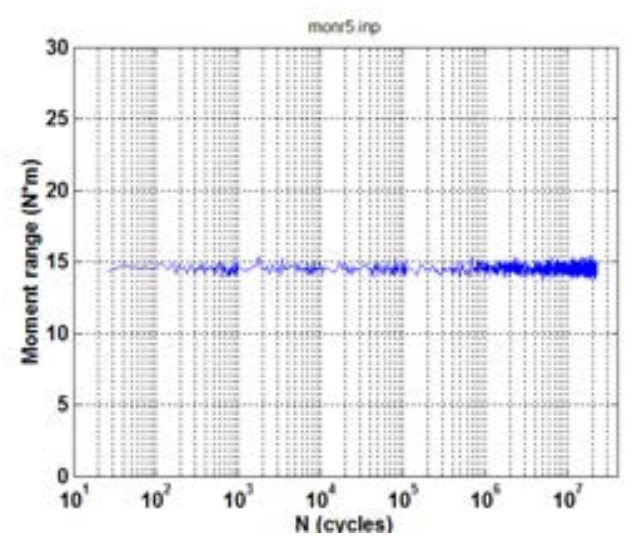

(b)

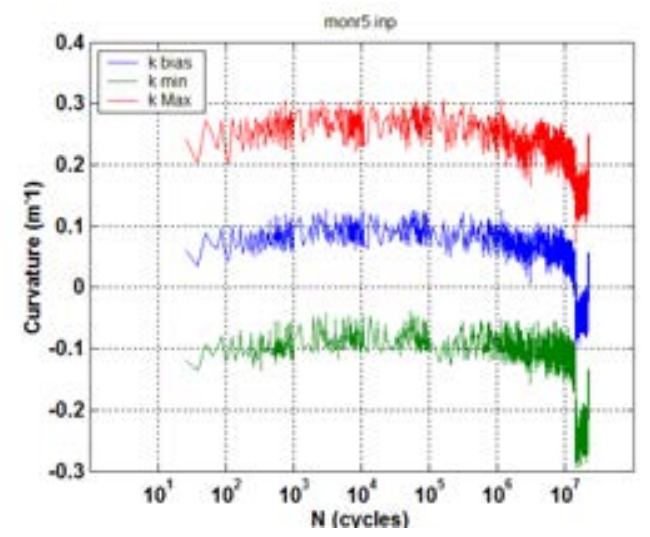

(d)

(e)

Figure E.37. Variations of (a) curvature range, (b) applied moment range, (c) flexural rigidity, (d) maximum and minimum values of curvature, and (e) maximum and minimum values of moment as a function of number of cycles for R5 (606B3C); $N=2.23 \times 10^{7}$ cycles under $\pm 7.62 \mathrm{~N} \cdot \mathrm{m} 5 \mathrm{~Hz}$. 


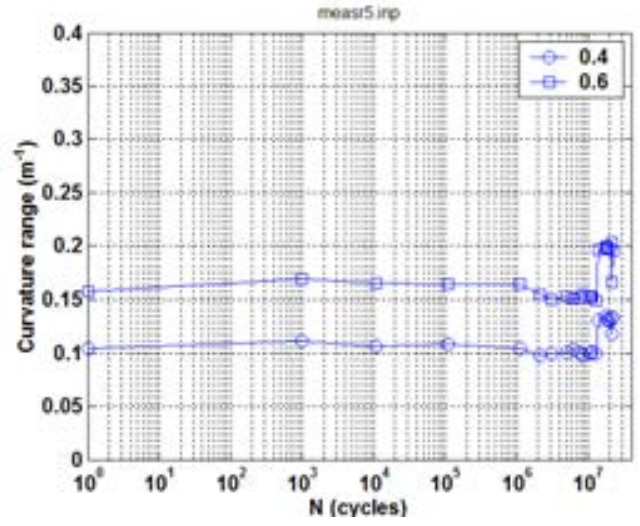

(a)

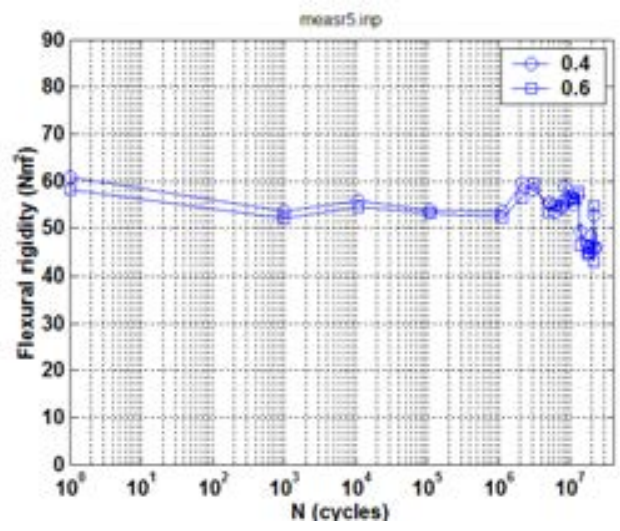

(c)

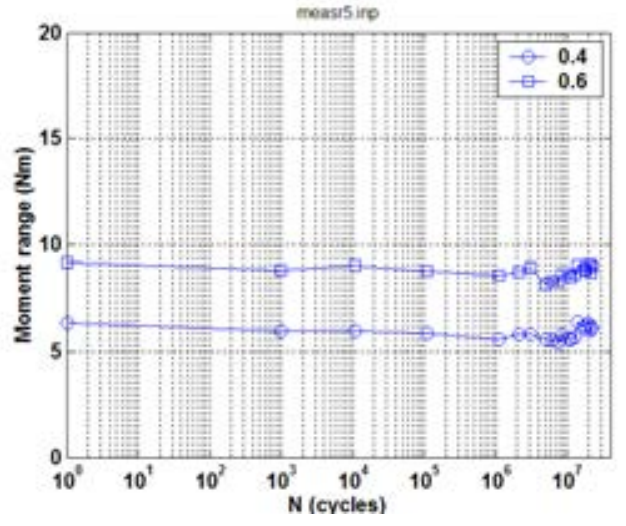

(b)

Figure E.38. Variations of (a) curvature range, (b) applied moment range, and (c) flexural rigidity as a function of number of cycles for $\mathrm{R} 5$ (606B3C); $\mathrm{N}=2.23 \times 10^{7}$ cycles under $\pm 7.62 \mathrm{~N} \cdot \mathrm{m} 5 \mathrm{~Hz}$. 


\section{APPENDIX F}

\section{POST IRRADIATION EXAMINATION (PIE)}





\section{PREPARATION OF SPECIMENS}

\section{Axial section}

PIE of the axial section was conducted on one untested rod segment, on a fractured specimen from static test S2, and on three specimens tested in dynamic tests [one un-failed (DM1) and two fractured (DL3 and DM2)].

For the tested and fractured specimen, the preparation of axial cross sections mainly involved the following steps: 1) cut a one-inch segment on the fracture end, 2) mount the segment with the bending plane in the horizontal direction and cut the top half away, 3) back-pot the remaining half to keep all the fuel in place, and 4) grind and polish the section to the required finish for metallography. Examinations were then performed on an optical microscope. ${ }^{18}$

The section preparation for other segments/specimens was similar to the above procedure. The untested rod segment was taken from a section of the same father fuel rod and with the same estimated amount of hydrogen content as those in other PIE specimens. The section for the tested and un-failed specimen was taken from the gage section of the specimen.

\section{Transverse section}

PIE on the transverse section was conducted only on D3.

The section was prepared by using an approximately 0.25 in. length of cladding cut away from the remaining half of specimen D3 (specimen holder A). The segment was defueled by using hot nitric acid. The defueled cladding was removed from the hot cell and cleaned with water and alcohol in an ultrasonic cleaner. The inside and outside surfaces of the clad were then coated with a thin layer of epoxy. The section was then ground and polished to the required finish for examination using scanning electron microscopy (SEM).

\section{UNTESTED SEGMENT 605D1D}

The untested section was prepared from one inch of fuel from segment 605D1D. Adjacent segments indicated that the estimated hydrogen content is around 400-500 ppm. An optical image of the section is given in Figure F.1 (a).

The three dished fuel pellets can be easily identified. Two primary fractures had developed approximately along and normal to the axial direction. Detail study [Figure F.1 (b)] showed that the pellet-to-pellet interface near the edge of the dish and the pellet-to-clad interface were fairly close without any visible gaps. Secondary fractures had developed within pellets near the peripheral area close to the pellet-to-clad interfaces. A cavity is also observed around some triple boundaries of pellets and clad. The circumferential hydride layers can be seen clearly over the whole thickness. The circumferential hydrides are widely spaced in the large middle part of the wall. The density of the hydrides increases significantly towards the outside of the clad and in the clad-pellet interface area. The circumferential hydride layers are very sizable and, in the middle of clad wall, the layers can run more than $1000 \mu \mathrm{m}$. The outside surface of clad is covered with a continuous thinner oxide layer that measured about $75 \mu \mathrm{m}$. 


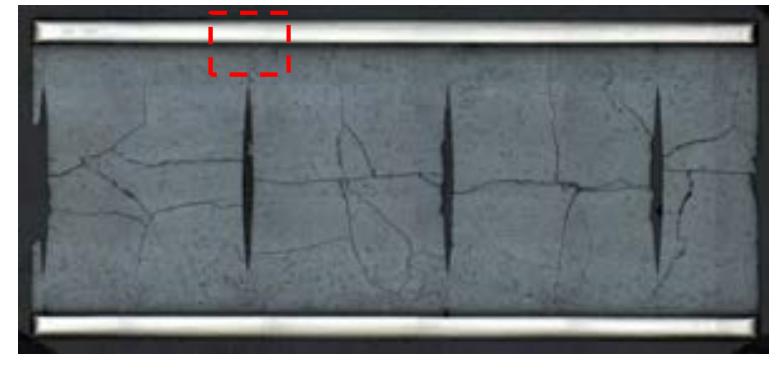

(a)

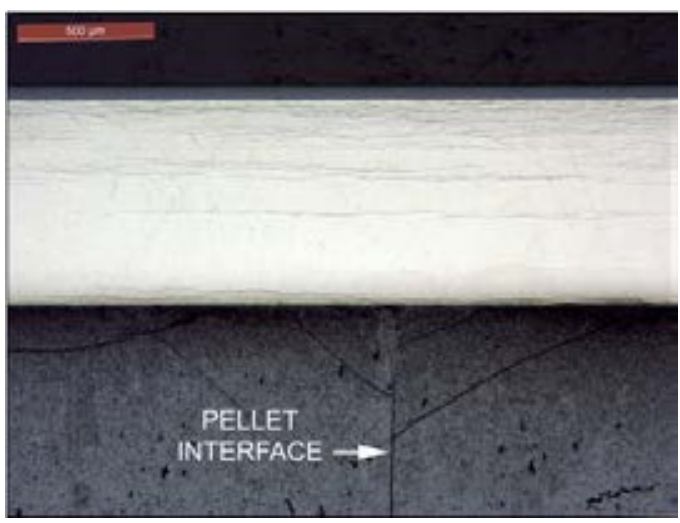

(b)

Figure F.1. Optical images for (a) overall axial cross section and (b) enlargement of area indicated in (a).

\section{FRACTURED IN STATIC TEST S2/ 605D1E}

S2 was based on segment 605D1E, whose estimated hydrogen and oxide layer thickness were $400 \mathrm{ppm}$ and $40-70 \mu \mathrm{m}$. S2 failed in a dynamic test $( \pm 30.48 \mathrm{~N} \cdot \mathrm{m}, 5 \mathrm{~Hz})$ after three cycles of unidirectional loading.

The failure took place at the pellet-to-pellet interface as seen in Figure F.2. Primary fractures in the same range of pellet size can be seen clearly. Unlike those in the untested fuel segment, the axial fractures did not align but tilted toward the axial direction.

The pellet-to-clad interface appeared in good contacts. The fracture surface in the clad exhibited a zigzag pattern both on the back and front sides of the specimen. This unique pattern resulted from the combination of brittle fracture of hydrides and ductile failure of the metal matrix under tension. Delamination can be seen over the hydride layers near the fracture surface.

In addition, spalling and cracking of the surface oxide layer can be seen on the back and front sides. 


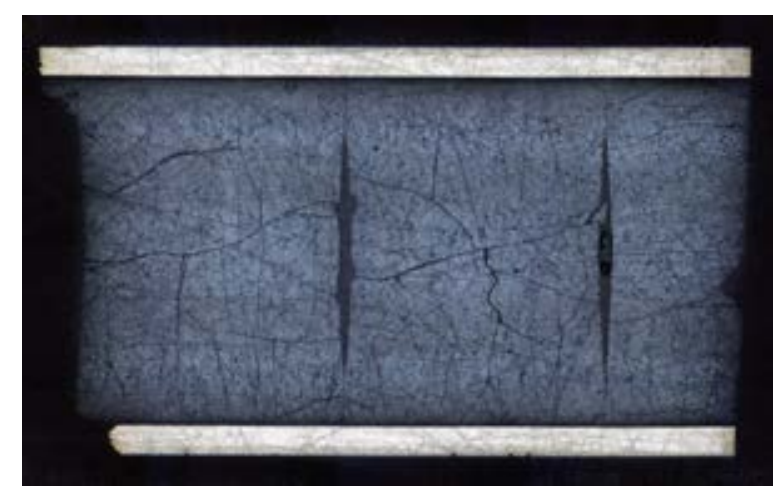

(a)
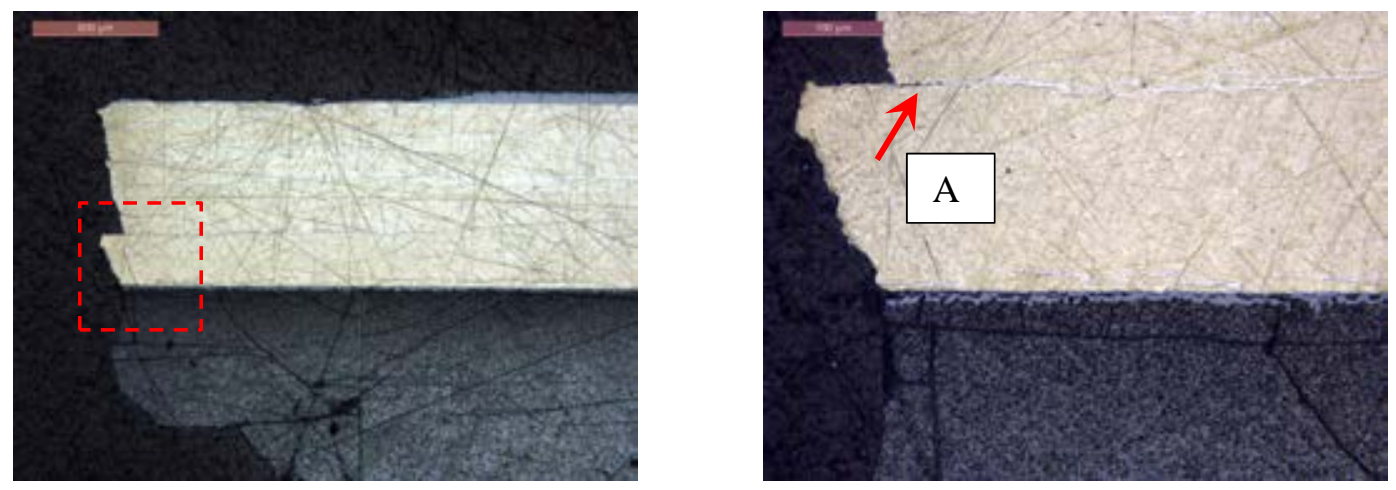

(b)
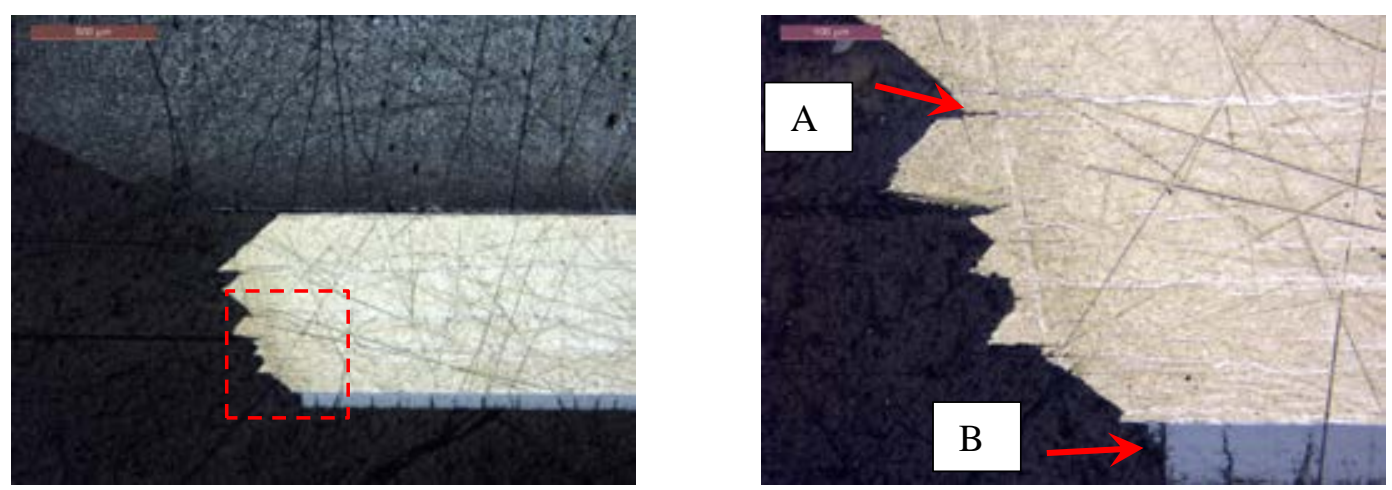

(c)

Figure F.2. (a) Axial cross section of S2/ 605D1E, (b) fracture surface near back side of rod and an enlarged area, and (c) fracture surface near front side of rod and an enlarged area. A - delamination; B - cracking of oxide layer.

\section{TESTED AND UN-FAILED IN DYNAMIC TEST D4/ DM1/ 605D1C}

D4/ DM1 was based on segment 605D1C, whose estimated hydrogen and oxide layer thickness were $500 \mathrm{ppm}$ and $40-70 \mu \mathrm{m}$. D4 survived $1.1 \times 107$ cycles of dynamic testing under $\pm 7.62 \mathrm{~N} \cdot \mathrm{m}$ $5 \mathrm{~Hz}$ without failure. 
The axial section of a one-inch segment is shown in Figure F.3. The primary fractures in the same range of pellet size can be seen clearly. The fracture size and fracture pattern were similar to those observed for the untested fuel segment. Also visible is that the closely spaced fractures interacted and resulted in slender fragments aligned in the axial direction. One of the fragments was pulled out in the grinding process.

The pellet-to-clad interface appeared to be in good contact. The circumferential hydrides are similar to those observed in the untested fuel segment. This is understandable because the two were neighboring segments in the same father fuel rod.

In addition, the surface oxide layer seems to be intact, even though the specimen experienced a high-cycle reversible bending fatigue test.

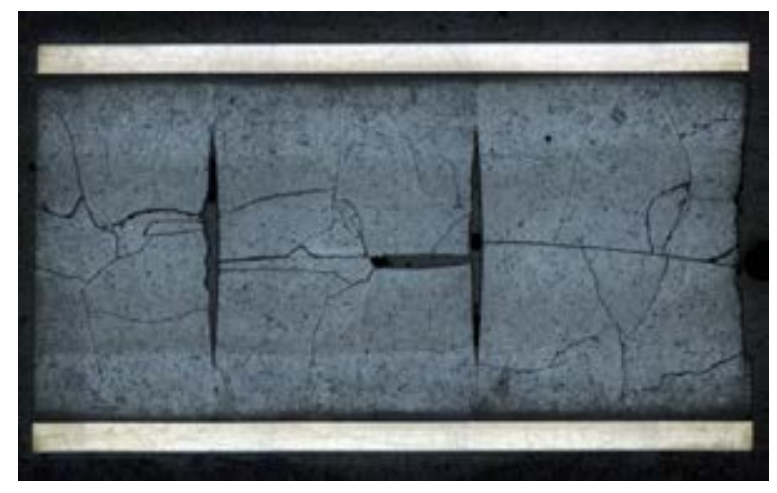

(a)

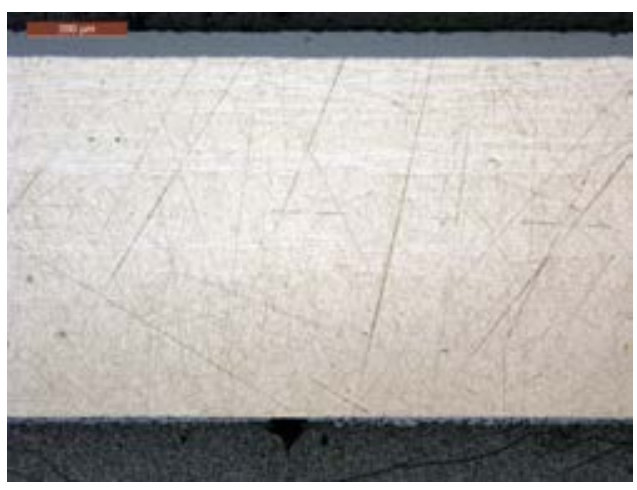

(b)

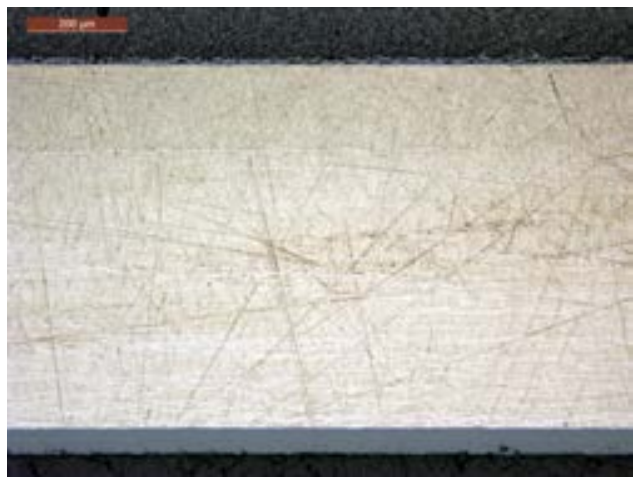

(c)

Figure F.3. (a) Axial cross section of DM1/ 605D1C, (b) back side of rod, and (c) front side of rod. 


\section{FRACTURED IN DYNAMIC TEST D3/ DL3/ 605C10A}

\section{Axial section}

D3/ DL3 was based on segment 605C10A with an estimated hydrogen contact of $550 \mathrm{ppm}$ and an oxide layer of $70-100 \mu \mathrm{m}$. As mentioned earlier, D3 was tested under $\pm 10.16 \mathrm{~N} \cdot \mathrm{m} 5 \mathrm{~Hz}$ with a lifetime of $10^{6}$ cycles.

An optical image of the axial cross section is given in Figure F.4. The fracture surface was located at the left side, or a pellet-to-pellet interface. It can be seen that the development of irradiation-induced primary fractures was similar to that of the specimens examined above.

Part of the clad fracture surface near the front side was not preserved. The fracture surface near the back side of the specimen was highly serrated. The cavity visible near the fracture perhaps resulted from hydride cracking because it is coincident with the circumferential hydride layers.

Spalling of the outside oxide layer was again seen near the fracture surface.

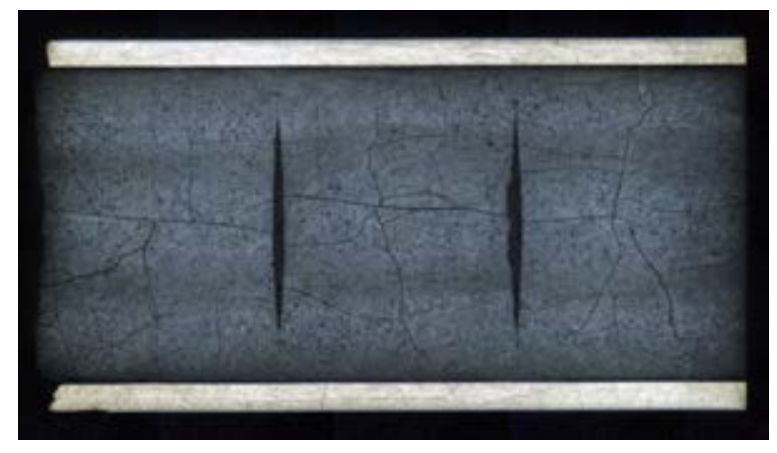

(a)

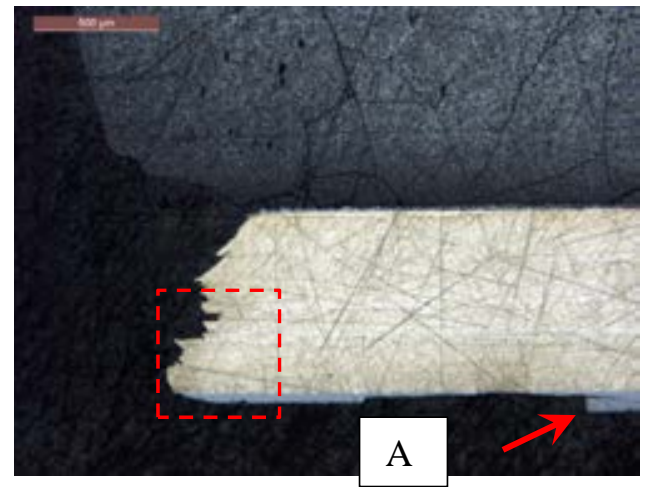

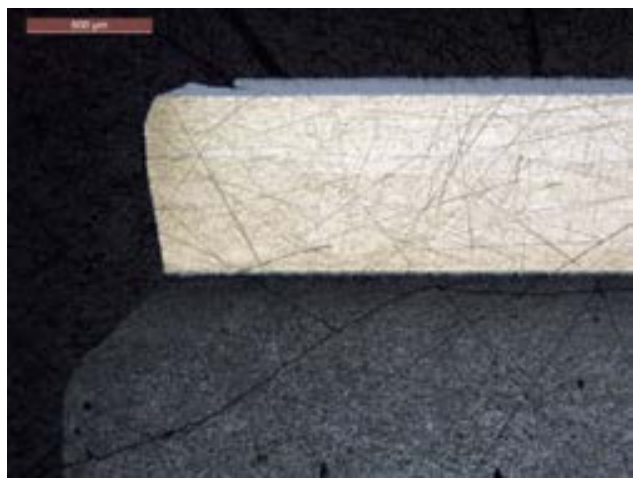

(b)

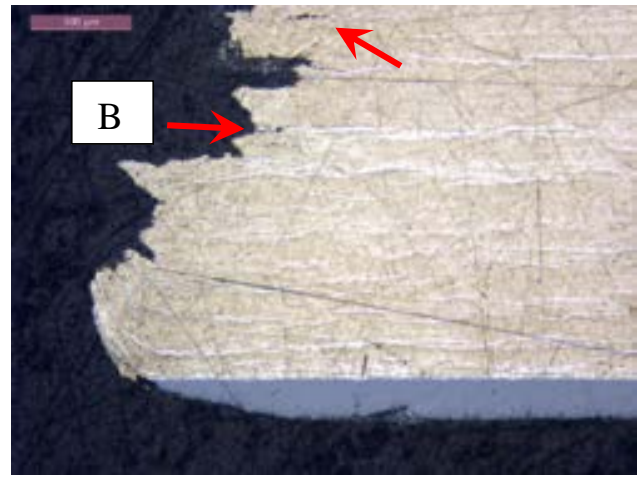

(c)

Figure F.4. (a) Axial cross section of D3/ 605C10A, (b) fracture surface near front side of rod, and (c) fracture surface near back side of rod and an enlarged area. A - spalling of oxide layer; B - cavity aligning with circumferential hydride layer. 


\section{Transverse section}

The set of SEM images presented in Figure F.5 (a-d) shows the different areas of the transverse cross section. The images corresponding to the 12 and 6 o'clock positions represent the maximum alternating stress areas of the rods subjected to potential crack initiation. The cladding was flattened apparently at the maximum stress areas after the fracture, and the origin of failure was not preserved. On the other hand, the areas near the 3 and 9 o'clock positions of the fracture surface revealed a clear laminate structure [Figure F.5 (e)] that may be related to the preexisting circumferential hydride structure in the HBU Zry-4 cladding.

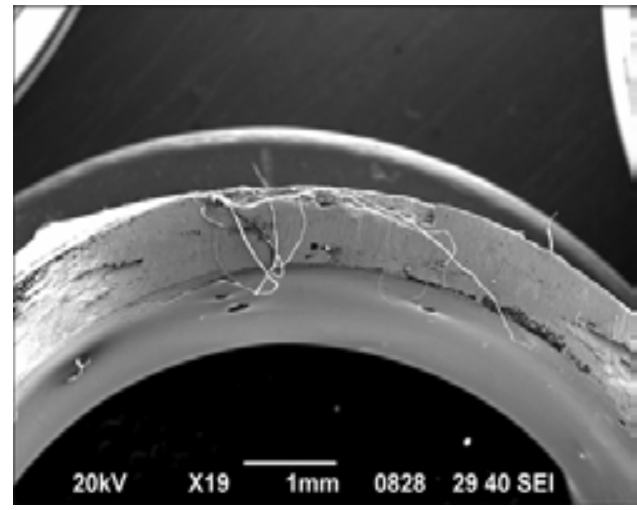

(a)

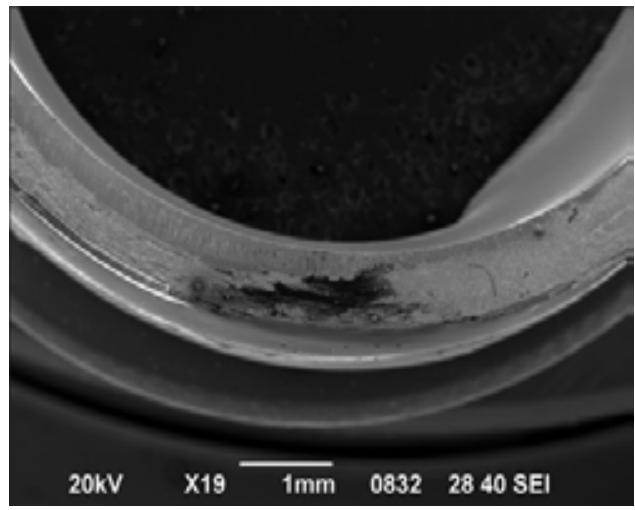

(c)

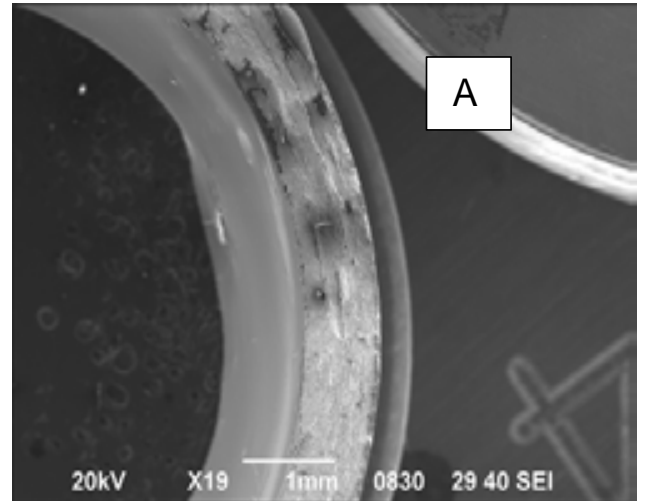

(b)

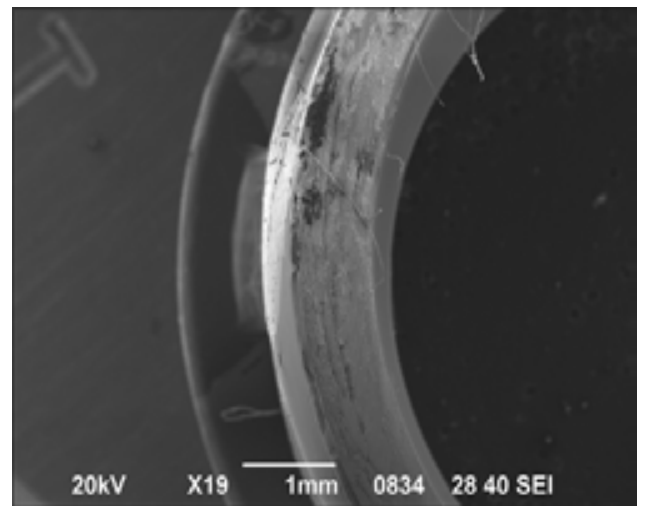

(d)

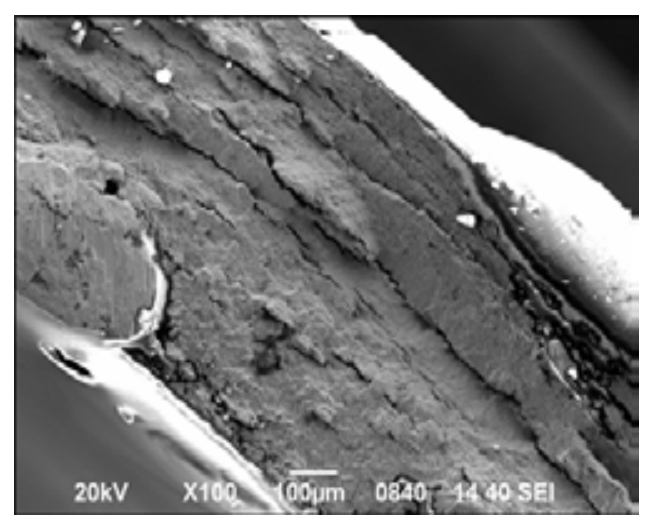

(e)

Figure F.5. SEM images of clad cross section based on the de-fueled D3: (a) 12 o'clock (reference mark), (b) 3 o'clock, (c) 6 o'clock, (d) 9 o'clock, and (e) enlarged area A. 


\section{FRACTURED IN DYNAMIC TEST D5I DM2/ 605D1B}

D5/ DM2 was based on segment 605D1B with an estimated hydrogen contact of $550 \mathrm{ppm}$ and an oxide layer of $40-70 \mu \mathrm{m}$. As mentioned earlier, D5 was tested under $\pm 9.14 \mathrm{~N} \cdot \mathrm{m} 5 \mathrm{~Hz}$ with a lifetime of $2.3 \times 10^{6}$ cycles.

An optical image of the axial cross section is given in Figure F.6. The fracture surface was located at the left side within a pellet. The primary fractures (whose size was of the same scale as the pellet) were similar to those revealed in the specimens examined above. The primary fractures were preexisting and may be a radiation effect because they were similar to those observed in untested fuel segments in terms of size and pattern. One primary fracture system normal to the axial direction of fuel corresponded to the fracture surface of the fuel and thus served as a main contributor to failure.

Part of the clad fracture surface near the front side was flattened, resulting from impacts to the fracture surfaces before the machine reached the predetermined termination condition. Part of the fracture surface near the back side of the specimen was preserved and exhibited a highly serrated appearance. The delamination and connected cavities can be seen near the fracture, which may be attributed to hydride cracking because they are coincident with the circumferential hydride layers.

At the same time, spalling of the outside oxide layer was seen near the fracture surface. 


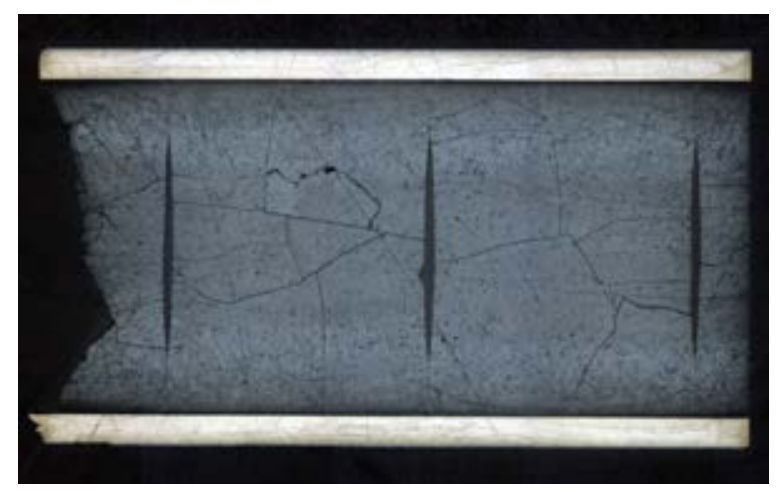

(a)

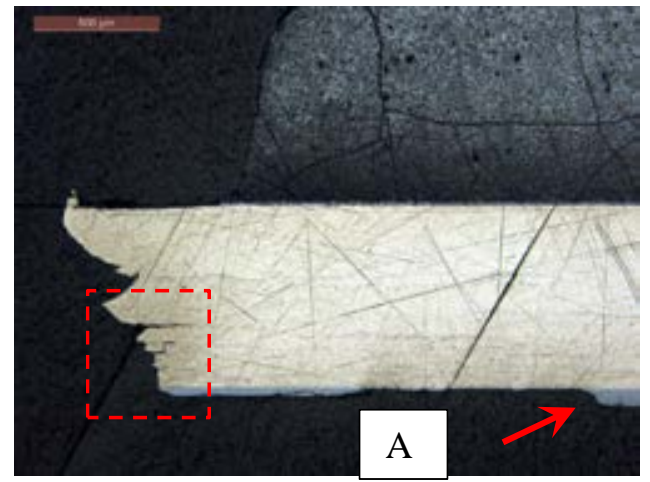

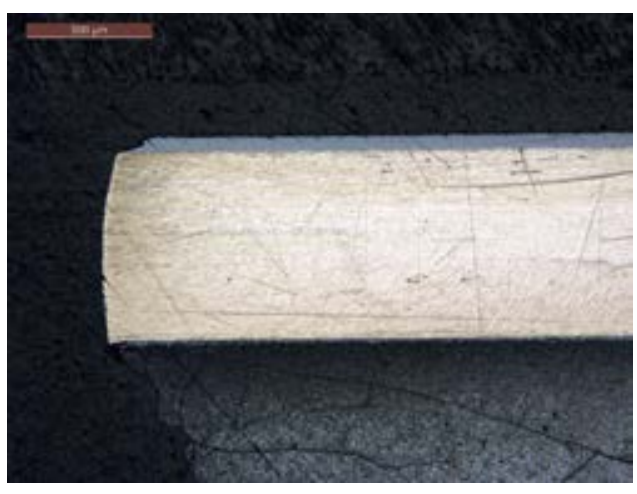

(b)

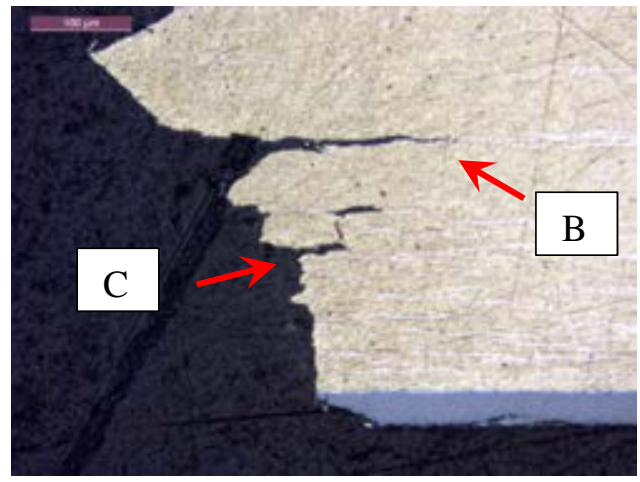

(c)

Figure F.6. (a) Axial cross section of D5/ 605D1B, (b) fracture surface near front side of rod, and (c) fracture surface near back side of rod and an enlarged area. A - spalling of oxide layer; B - delamination; C - connected cavities aligning with hydride layers. 




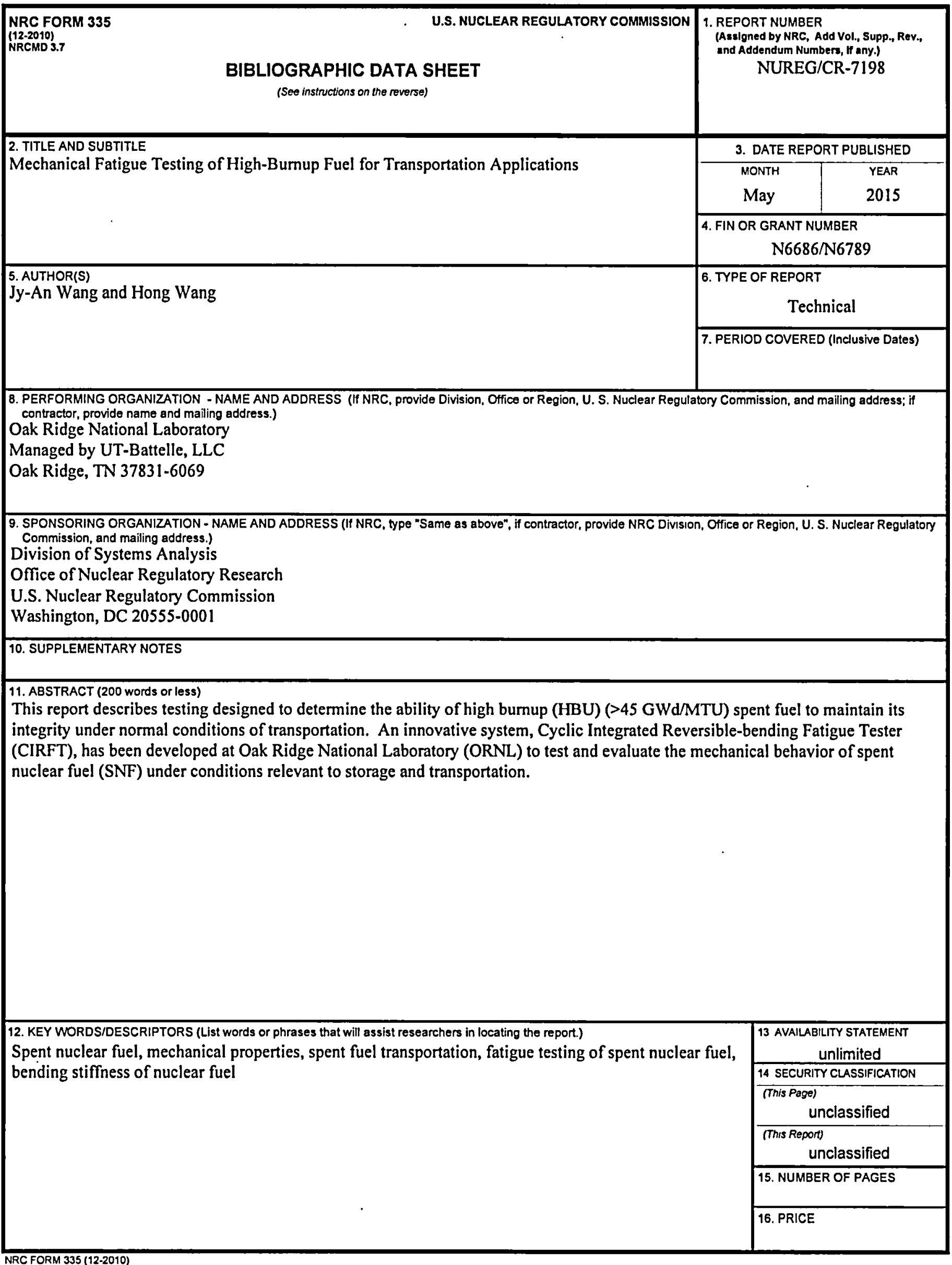




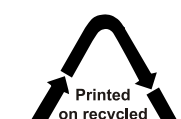

Printed
on recycled
paper

paper

Federal Recycling Program 



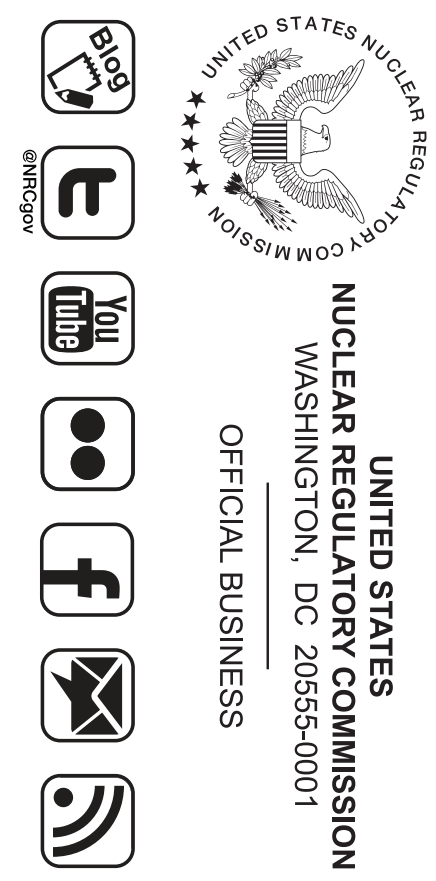



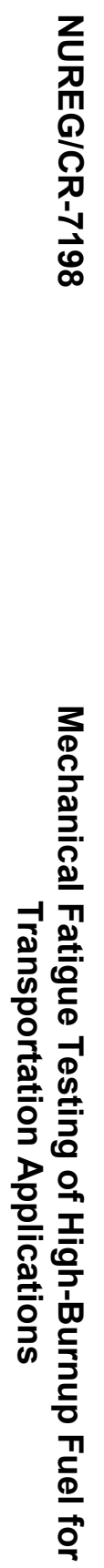

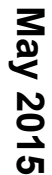

